\title{
Finite-time stability tools for control and estimation
}

Suggested Citation: Denis Efimov and Andrey Polyakov (202x), "Finite-time stability tools for control and estimation", : Vol. xx, No. xx, pp 1-xx. DOI: 10.1561/XXXXXXXXX.

\author{
Denis Efimov \\ Inria, Lille, France \\ denis.efimov@inria.fr \\ Andrey Polyakov \\ Inria, Lille, France \\ andrey.polyakov@inria.fr
}




\section{Contents}

1 Introduction and motivation 2

1.1 General terminology . . . . . . . . . . . . . . . . . 2

1.2 Historical remarks . . . . . . . . . . . . . . . . . 3

1.3 Motivating examples . . . . . . . . . . . . . . . 4

1.4 The structure of the paper . . . . . . . . . . . 15

$\begin{array}{ll}\text { I Analysis } & 17\end{array}$

2 Notions of interest $\quad 19$

2.1 Definitions of finite-time and fixed-time stability . . . . 20

2.2 Robust stability in the presence of disturbances . . . . . 34

2.3 Discussions . . . . . . . . . . . . . . . . . . . . 40

3 Homogeneity 42

3.1 Definitions and various concepts . . . . . . . . . . . . . 43

3.2 Properties of homogeneous systems . . . . . . . . 55

3.3 Stability Analysis . . . . . . . . . . . . . . . . 57

3.4 Robustness . . . . . . . . . . . . . . . . . . . . 61

3.5 Homogeneous approximations . . . . . . . . . . . . . . 62

3.6 Homogeneity in Hilbert spaces . . . . . . . . . . . . 66 
4 Control Design $\quad 84$

4.1 Finite/Fixed-Time stabilization of continuous affine systems 85

4.2 Homogeneous stabilization of linear plants . . . . . . . 89

4.3 Homogeneous stabilization of nonlinear plants . . . . . 96

5 State Estimation $\quad 101$

5.1 Problem statement . . . . . . . . . . . . . . . 103

5.2 Observer design for a homogeneous linear plant . . . . . . 103

5.3 On selection of observer's parameters . . . . . . . . 105

5.4 Necessary and sufficient condition of homogeneous observability . . . . . . . . . . . . . . 106

5.5 Robustness Analysis . . . . . . . . . . . . . . . . 107

5.6 Homogeneous differentiator . . . . . . . . . . . . . 108

III Discretizations and extensions to infinite dimensional systems

6 Implementation and discretization

6.1 Discretization of homogeneous dynamics . . . . . . . . . 112

6.2 Digital Implementation of ILF-based algorithms . . . . . . 142

7 Extensions

7.1 Time-delay systems . . . . . . . . . . . . . . . . . . . 153

7.2 Partial differential equations and evolution models . . . . 166

Appendices

A Notation

References 


\title{
Finite-time stability tools for control and estimation
}

Denis Efimov ${ }^{1}$ and Andrey Polyakov ${ }^{2}$

${ }^{1}$ Inria, Lille, France; denis.efimov@inria.fr

${ }^{2}$ Inria, Lille,France; andrey.polyakov@inria.fr

\begin{abstract}
This survey presents some existing and new results on analysis and design of finite-time and fixed-time converging systems. Two main groups of approaches for analysis/synthesis of this kind of convergence are considered: based on Lyapunov functions and the theory of homogeneous systems. The focus is put on the dynamics described by ordinary differential equations, time-delay models and partial differential equations. Some popular control and estimation algorithms, which posses accelerated converge rates, are reviewed. The issues of discretization of finite-/fixed-time converging systems are discussed.
\end{abstract}

Denis Efimov and Andrey Polyakov (202x), "Finite-time stability tools for control and estimation", : Vol. xx, No. xx, pp 1-xx. DOI: 10.1561/XXXXXXXXX. 


\section{Introduction and motivation}

\subsection{General terminology}

To design an estimation or a control algorithm we have to select performance criteria to be optimized. Stability is one of the main quality indexes, which is usually investigated with respect to an invariant mode (e.g., an equilibrium, desired trajectory or a set), so another important characteristics is the time of convergence of the system trajectories to this mode, which can be infinite (in linear time-invariant systems) or finite. In the latter case the limit mode has to be exactly established in a finite time (dependent on initial deviations). If such a time is independent on initial conditions, then this kind of convergence is called fixed-time. The notion of finite-time stability has been proposed in 60s by Emilo Roxin (Roxin, 1966) and it has been developed in many works later, where a particular attention is paid to the time of convergence to a steady state. The fixed-time stability concept is quite recent (Polyakov, 2012). Many results on analysis and design of control and estimation algorithms in this context have been obtained, which profit from a fine development of the Lyapunov function method to these sorts of stability. Another useful and simple method to deal with finite- or fixed-time stability is based on the theory of dynamical homogeneous systems. 
Homogeneity is a property of mathematical objects, such as functions or vector fields, to be scaled in a consistent manner with respect to a scaling operation (called a dilation) applied to their argument. Such a type of symmetry is defined in a way leading to the scaling of trajectories of corresponding dynamical systems. So homogeneous systems possess several important and useful properties: their local behavior is the same as global one, the rate of convergence to the origin can be identified by degree of homogeneity (a parameter of symmetry), the stability is robust to various perturbations. The first rise of homogeneity consists in homogeneous polynomials investigated by Euler in 18th century. In 50s and 60s more generic notions of homogeneity (weighted and coordinatefree or geometric) have been introduced by Vladimir Zubov and his group (see, Zubov, 1964; Khomenuk, 1961 and references therein). Many other studies were performed in the last decades, and these notions of homogeneity have been extended to other kinds of systems (discontinuous models, time-delay systems, partial differential equations, discrete-time models).

\subsection{Historical remarks}

The concept of asymptotic stability introduced by the famous thesis of A.M. Lyapunov (1892) is one of central notions of the modern control theory. Many problems of state estimation and control can be reduced to a stability analysis or to a stabilization of solutions of certain dynamical models. Being the very profound and fundamental concept, the classical notion of the asymptotic stability does not characterize a convergence time (known as a time response in control theory) of a nonlinear system, and in such a case it is implicitly assumed that the desired mode is reached when $t \rightarrow+\infty$. In Erugin, 1951, it was mentioned that certain asymptotically stable systems may reach a stable equilibrium in a finite time. This time can be considered as a quantitative characteristic of the time response of a control system. The notion of finite stability (also known today as finite-time stability, see Bhat and Bernstein, 2000) has been introduced in Roxin, 1966.

In control theory, finite-time convergence to a set-point was studied in the context of the so-called minimum time control problem Feld- 
baum, 1953. The corresponding optimal solution can be obtained in the feedforward form according the celebrated Feldbaum's theorem about $n$ intervals. In Fuller, 1960 it was discovered that some optimal control problems has solutions in the form of finite-time stabilizing relay feedbacks. The sliding mode control theory still uses the finite-time stabilization properties of relay feedbacks for enforcing a sliding motion Utkin, 1992, Shtessel et al., 2014. In a more systematic way, the problem of finite-time feedback stabilization has been studied in Haimo, 1986 for planar systems. In Korobov, 1979 and Korobov, 1980 the problem of a finite-time stabilization with a bounded control magnitude has been studied for linear and nonlinear plants using the so-called controllability function method. Relations of finite-time stability and controllability of nonlinear systems were also studied in Kawski, 1989 and Coron and Praly, 1991.

Being the main tool for stability analysis of nonlinear systems, the Lyapunov function method is utilized for a characterization of the finite-time stability since Roxin, 1966. However, the "modern era" of finite-time stability and stabilization in control was initiated in Bhat and Bernstein, 2000, where the Lyapunov function method for finite-time stability analysis was refined. In the last 20 years, finite-time control and estimation algorithms were developed for linear/nonlinear and finite/infinite dimensional models. The aim of this paper is to survey some existing tools for finite-time stability analysis and finite-time controllers/observers design.

\subsection{Motivating examples}

Initially, we would like to consider few motivating examples, where finite-time stability and stabilization can be discovered in system models and/or utilized for control design purposes.

\subsubsection{Torricelli's law}

This law of fluid dynamics, which was discovered by an Italian scientist Evangelista Torricelli in 1643, relates the velocity of fluid flowing from an orifice to the height of fluid above the opening. The law establishes 
that the speed of a fluid $v$ exiting through a hole, located at the bottom of the tank and filled by the liquid till the level $h$, equals

$$
v=\sqrt{2 g h},
$$

where $g$ is the acceleration due to the gravity (there are also mild assumptions on the properties of the orifice and the liquid). This expression can be obtained by equating the gained kinetic energy, $\frac{m v^{2}}{2}$, with the lost potential energy, $m g h$, and solving it for $v$.

The Torricelli's law can be further used to determine the dynamics of decay of the liquid's level $h(t)$ in the tank, which with the escaping water naturally goes in time $t \geq 0$ from the initial value $h(0)>0$ till zero. To this end, assume that the tank is cylindrical with a fixed cross-sectional area $A=\pi r^{2}$, where $r>0$ is the radius of the tank, and the cross-section of the hole located at the bottom of the tank is $a>0$, then the rate of outflow can be obtained as

$$
A \frac{d h(t)}{d t}=-a v(t)=-a \sqrt{2 g h(t)}
$$

while $h(t)>0$. Hence,

$$
\frac{d h(t)}{d t}=-\frac{a}{A} \sqrt{2 g h(t)}
$$

and the direct computations show that $h(T)=0$ in a finite time $T=\frac{A}{a} \sqrt{\frac{2}{g} h(0)}$. Such a finite-time escape of water from a volume is everybody's basic experience, which was used by humans in engineering during centuries as, for example, in clepsydra (that is a clock measuring the time using the flow of water).

\subsubsection{Mechanical models with dry and viscous frictions}

Let us consider a mechanical system consisting of a rigid body moving laterally on a contact surface and in a viscous environment (fluid). The simplest real-life example of such a mechanical system is a car moving on a flat road.

Let $z(t)$ be the position of the center of mass of the body in an inertial frame at time $t \in \mathbb{R}$. The equation describing a motion of this system has the form

$$
\dot{z}(t)=v(t), \quad m \dot{v}(t)=F(t), \quad t>0, \quad z(t) \in \mathbb{R},
$$


where $v(t)$ is the velocity, $m$ is the mass of the body, and $F$ is the sum of external forces.

Let us consider only the deceleration phase of the motion assuming that at the initial instant of time this mechanical system has some non-zero velocity $\dot{z}(0)=v(0) \neq 0$. Dissipation of the energy is caused, basically, by two external forces:

- the drag force (fluid resistance) is proportional to the velocity squared (see Falkovich, 2011)

$$
F_{\text {drag }}(t)=-k_{\text {drag }} v^{2}(t) \operatorname{sign}(v(t)),
$$

where $k_{d r a g}>0$ is the coefficient of fluid (air) resistance and the sign function is given by

$$
\operatorname{sign}(\rho)=\left\{\begin{array}{ccc}
1 & \text { if } & \rho>0 \\
0 & \text { if } & \rho=0, \\
-1 & \text { if } & \rho<0
\end{array}\right.
$$

- the dry friction force is nearly independent of the velocity and can be modeled as follows (see ,e.g., Armstrong-Helouvry, 1991)

$$
F_{d r y}(t)=-k_{d r y} \operatorname{sign}(v(t)),
$$

where $k_{d r y}>0$ is the coefficient of dry friction.

A more general friction model also may contain some linear terms (proportional to the velocity). We skip them for simplicity of analysis, since they will not change any conclusion about convergence rates of the system.

The sum of external forces $F(t)$ can be represented as follows

$$
F(t)=F_{d r a g}(t)+F_{d r y}(t)=-\left(k_{d r y}+k_{d r a g} v^{2}(t)\right) \operatorname{sign}(v(t)) .
$$

and the differential equation describing an evolution of the velocity of the body has the form:

$$
m \dot{v}(t)=-\left(k_{d r y}+k_{d r a g} v^{2}(t)\right) \operatorname{sign}(v(t)) .
$$

It is not difficult to show that $v=0$ is the equilibrium of the latter equation, which is globally asymptotically stable, $v(t) \rightarrow 0$ as $t \rightarrow+\infty$. 
The solution of this ODE can be found explicitly :

$$
v(t)=\tan \left(\arctan (|v(0)|)-\frac{\sqrt{k_{d r y} k_{d r a g}}}{m} t\right) \operatorname{sign}(v(0)) .
$$

This immediately implies $v(t)=0$ for $t \geq \frac{m \arctan (|v(0)|)}{\sqrt{k_{d r y} k_{d r a g}}}$. The function arctan is globally uniformly bounded. We conclude that independently of the initial velocity, the motion of the body terminates no later than the following instant of time

$$
T_{\max }=\frac{m \pi}{2 \sqrt{k_{d r y} k_{d r a g}}} .
$$

This property is also known as the fixed-time stability (or the fixed-time convergence).

\subsubsection{Extinction and blow-up in heat equation}

The finite-time operations are omnipresent in the complex dynamical processes described by PDE, and for an example let us consider a reaction-diffusion equation:

$$
\frac{\partial u(t)}{\partial t}=\Delta u(t)+\varphi(u(t)), \quad t>0, \quad u(0)=u_{0} \in L^{2}(\mathcal{X}, \mathbb{R}),
$$

where $\mathcal{X} \subset \mathbb{R}^{n}$ is an open connected set with a smooth boundary; $u(t) \in L^{2}(\mathcal{X}, \mathbb{R})$ is the state of the system supported on $\mathcal{X} ; t \in \mathbb{R}_{+}$is the time variable; the function $u_{0} \in L^{2}(\mathcal{X}, \mathbb{R})$ defines the initial state. The Laplace operator $\Delta=\nabla \cdot \nabla$,

$$
\Delta: \mathcal{D}(\Delta) \subset L^{2}(\mathcal{X}, \mathbb{R}) \rightarrow L^{2}(\mathcal{X}, \mathbb{R}), \quad \mathcal{D}(\Delta)=H_{0}^{1}(\mathcal{X}, \mathbb{R}) \cap H^{2}(\mathcal{X}, \mathbb{R})
$$

stands for a characterization of a diffusion, while the function

$$
\varphi: L^{2}(\mathcal{X}, \mathbb{R}) \rightarrow L^{2}(\mathcal{X}, \mathbb{R})
$$

expresses reaction/absorption. Let us consider the reaction-diffusion dynamics with a particular nonlinearity:

$$
\varphi(u)=k\|u\|_{L^{2}}^{p} u
$$

with $k \in \mathbb{R}$ and $p>-1$. In this case, the function $\phi$ is continuously differentiable on $L^{2}(\mathcal{X}, \mathbb{R}) \backslash\{\mathbf{0}\}$, so the system has a unique classical 
solution $u \in C^{1}\left([0, T), L^{2}(\mathcal{X}, \mathbb{R})\right): u(t) \in \mathcal{D}, \forall t>0$ for any initial state $\mathbf{0} \neq u_{0} \in \mathcal{D} \backslash\{\mathbf{0}\}$ (see, Pazy, 1983, page 187). For $u_{0}=\mathbf{0}$ the system has the zero solution, which may be non-unique in the general case. Depending on the selection of parameters in the heat equation, a finitetime blow-up can be observed for $k>0$ and $p>0$ : that is existence of a finite $T_{f}>0$ such that

$$
\lim _{t \rightarrow T_{f}}\|u(t)\|_{L^{2}}=+\infty,
$$

or a finite-time extinction/absorption can be obtained for $k<0$ and $p \in(-1,0)$ :

$$
\lim _{t \rightarrow T_{f}}\|u(t)\|_{L^{2}}=0
$$

for some finite $T_{f}>0$; finally, if $k<0$ and $p>1$, a uniform convergence to a ball in $L^{2}$ is recovered

$$
\lim _{t \rightarrow T_{f}}\|u(t)\|_{L^{2}} \leq 1
$$

for any $u_{0} \in \mathcal{D}$ and some $T_{f}>0$. The presented conclusions follow from the identity

$$
\frac{1}{2} \frac{d}{d t}\|u(t)\|_{L^{2}}^{2}=-\|\nabla u(t)\|_{L^{2}}^{2}+k\|u(t)\|_{L^{2}}^{p+2}, \quad t>0,
$$

which holds for any classical solution of the considered heat equation.

\subsubsection{Minimum time control problem and sliding mode algorithms}

A finite-time control algorithm appears in control theory, for example, as solution of the classical minimum time control problem

$$
T \rightarrow \min _{u}
$$

subject to

$$
\begin{cases}\dot{x}_{1}=x_{2}, & u \in L^{\infty}((0, T), \mathbb{R}) \text { such that }|u(t)| \leq 1 \\ \dot{x}_{2}=u, & x_{1}(T)=x_{2}(T)=0,\end{cases}
$$

Indeed (see, e.g., Chernous'ko et al., 2008), the optimal feedback has the form

$$
u=-\operatorname{sign}\left(\left|x_{2}\right| x_{2}+2 x_{1}\right),
$$

which is, in fact, the so-called high order sliding mode controller Levant, 2005, which stabilize the origin of the system in a finite time. 


\subsubsection{Estimation for hybrid systems}

In many engineering applications the decisions have to be taken and implemented in a finite time, which may be related with system reconfiguration (e.g., destruction, or disappearance of control/measurements) after a time period. A generic example of such a case is the problem of state estimation (similar consideration can be repeated for the control design) in hybrid systems (or system with impacts or impulses), which can be modeled as

$$
\begin{aligned}
\dot{x}(t) & =F(t, x(t)) & & x(t) \in C, \\
x\left(t^{+}\right) & =G(t, x(t)) & & x(t) \in D, \\
y(t) & =H(x(t)), & &
\end{aligned}
$$

where $x(t) \in \mathbb{R}^{n}$ is the state vector, $t \geq 0$; the map $F: \mathbb{R}_{+} \times \mathbb{R}^{n} \rightarrow \mathbb{R}^{n}$ determines the flow of the system while the movement are continuous and belong to the set $C \subseteq \mathbb{R}^{n}$, while $G: \mathbb{R}_{+} \times \mathbb{R}^{n} \rightarrow \mathbb{R}^{n}$ defines the jump or impact dynamics when $x(t)$ enters into the set of discontinuity $D \subseteq \mathbb{R}^{n} ; y(t) \in \mathbb{R}^{p}$ is the output available for measurements. Assume that the form of $C, D$ and the properties of $F, G$ are such that there is no Zeno behavior, i.e., the instants of jumps are always separated in time, and that the solution $x(t)$ of the system is unique and well-defined for any initial condition $x(0) \in \mathbb{R}^{n}$. It is required to estimate the current state value $x(t)$ using the measured information $y(t)$ and the knowledge about the maps $F, G$ and $H$, and about the sets $C$ and $D$.

Note that to design a conventional estimator, which is constituted by the copy of the original dynamics with an output injection in order to adjust the estimate $\hat{x}(t) \in \mathbb{R}^{n}$ :

$$
\begin{array}{rlrl}
\dot{\hat{x}}(t) & =F(t, \hat{x}(t))+L_{C}(y(t)-H(\hat{x}(t))) & \hat{x}(t) & \in C, \\
\hat{x}\left(t^{+}\right) & =G(t, \hat{x}(t))+L_{D}(y(t)-H(\hat{x}(t))) & \hat{x}(t) \in D,
\end{array}
$$

where $L_{C}, L_{D} \in \mathbb{R}^{n \times p}$ are the observer gains (for simplicity consider a basic linear correction), it is necessary to make the convergence analysis for the resulting hybrid (nonlinear) dynamics of the estimation error $e(t)=x(t)-\hat{x}(t)$. Such an investigation is rather sophisticated, and if the shape of set $D$ depends on unmeasured variables, then it may be hard to ensure the quality of estimation. 
Another solution consists in design of a fast observer, that always converges before the impact or reconfiguration. If the original system has an asymptotic convergence rate, then finite-time decay of $e(t)$ solves the problem. If a fixed-time convergence of $e(t)$ to zero is guaranteed, then uniformity in the uncertainty on initial conditions and the impact influence is provided.

\subsubsection{Elimination of the unbounded peaking effect}

Any controllable linear system can be stabilized at the origin by means of a static linear feedback. The time of convergence of trajectories from the unit ball into a neighborhood of the origin can be prescribed in advance by means of an appropriate tuning of the feedback gain. Such a stabilization is sufficient for many practical problems. The reasonable question in this case: Is there any advantage of a nonlinear finite-time stabilizing controller comparing with the classical linear feedback?

Let us consider the control system

$$
\dot{x}=A x+B u(x), \quad t>0, \quad A=\left(\begin{array}{ccccc}
0 & 1 & 0 & \cdots & 0 \\
0 & 0 & 1 & \cdots & 0 \\
\cdots & \cdots & \cdots & \cdots & 0 \\
0 & 0 & 0 & \cdots & 1 \\
0 & 0 & 0 & \cdots & 0
\end{array}\right), \quad B=\left(\begin{array}{c}
0 \\
0 \\
\cdots \\
0 \\
1
\end{array}\right)
$$

where $x=\left(x_{1}, x_{2}, \ldots, x_{n}\right)^{\top}$ is the state vector and $u: \mathbb{R}^{n} \rightarrow \mathbb{R}$ is the feedback control. Initial conditions of the latter system are assumed to be bounded as follows

$$
\|x(0)\| \leq 1 \text {. }
$$

The control aim is to stabilize the state vector $x(t)$ of the system into a ball of a small radius $\varepsilon>0$ in a prescribed time $T>0$ :

$$
\|x(t)\| \leq \varepsilon, \quad \forall t \geq T .
$$

Let us consider the static linear feedback

$$
u_{\ell}(x):=k x, \quad k=\left(k_{1}, k_{2}, \ldots, k_{n}\right) .
$$

The eigenvalues $\left\{\lambda_{1}, \ldots, \lambda_{n}\right\}$ of the closed-loop linear system

$$
\dot{x}=(A+b k) x
$$

can be placed in any given set of the complex plane $\mathbb{C}$ by choosing the vector $k$ (see, e.g., Wonham, 1985). Therefore, it is possible to obtain a 
closed-loop system with an arbitrary fast damping speed, i.e.

$$
\forall \varepsilon>0, \quad \exists k \in \mathbb{R}^{1 \times n}: \sup _{\left\|x_{0}\right\|=1}\|x(t)\|<\varepsilon, \quad t>T .
$$

Indeed, trajectories of this system converge to the origin exponentially fast

$$
\|x(t)\| \leq C e^{-\sigma t}, t>0
$$

where the constant $C \geq 1$ depends on $\lambda_{i}, i=1,2, . ., n$ and $\Re\left(\lambda_{i}\right)<-\sigma$. Hence, smaller $\varepsilon>0$ larger $\sigma>0$ has to be assigned to solve the control problem, i.e. $\sigma \rightarrow+\infty$ as $\varepsilon \rightarrow 0$ provided that $T$ is fixed. Therefore, we conclude that the linear state feedback is, indeed, a possible solution of the considered stabilization problem for any fixed $\varepsilon>0$.

However, the trajectories of the closed-loop linear system with fast decays have large deviations from the origin during the initial phase of the stabilization (the constant $C$ depends directly on $\sigma$ and inversely on $\varepsilon$ ). This phenomenon is called the "peaking" effect and the large deviation is referred to as an "overshoot" (see Polyak and Smirnov, 2016 for more details). In particular, it is shown (Izmailov, 1987) that there exists $\gamma>0$ independent of $\lambda_{i}$ such that

$$
\sup _{0 \leq t \leq \sigma^{-1}} \sup _{\|x(0)\|=1}\|x(t)\| \geq \gamma \sigma^{n-1} .
$$

Hence, we immediately conclude that a larger $\sigma>0$ leads to a larger "overshoot" in a shorter time.

For $n>1$ the linear closed-loop system has an infinite "overshoot" as $\varepsilon \rightarrow 0$ :

$$
\sup _{0 \leq t \leq T} \sup _{\|x(0)\|=1}\|x(t)\| \rightarrow+\infty \quad \text { as } \quad \varepsilon \rightarrow 0 .
$$

This means that for a sufficiently small $\varepsilon>0$ the linear system may have so huge "overshoot" that practical (e.g., physical) restrictions to the system states would not allow it. The static linear control needs to be somehow modified to overcome this difficulty. The simplest way is to use some input saturation, which, in fact, anyway must be taken into account in practice. However, in this case it is not clear if the saturated feedback would solve the considered stabilization problem 
with the prescribed time $T>0$ provided that the saturation would not destroy stability of the system trajectories initiated in the unit ball.

Another possible way to eliminate the infinite "peaking" effect is a transformation of the linear controller to a finite-time controller. Indeed, let us consider the following feedback law

$$
u_{h}(x)=\tilde{k} \mathbf{d}\left(-\ln \|x\|_{\mathbf{d}}\right) x,
$$

where $\mathbf{d}$ is the weighted dilation

$$
\mathbf{d}(s)=\left(\begin{array}{cccc}
e^{n s} & 0 & \ldots & 0 \\
0 & e^{(n-1) s} & \ldots & 0 \\
\cdots & \ldots & \ldots & \ldots \\
0 & 0 & \cdots & e^{s}
\end{array}\right), \quad s \in \mathbb{R}
$$

and $\|\cdot\|_{\mathbf{d}}: \mathbb{R}^{n} \rightarrow(0,+\infty)$ is the so-called canonical homogeneous norm studied in Chapter 3.

In Chapter 4 it is shown that the vector $\tilde{k}=\left(\tilde{k}_{1}, \tilde{k}_{2}, \ldots, \tilde{k}_{n}\right)^{\top}$ can be easily selected to guarantee

$$
\sup _{\|x(0)\|=1}\|x(t)\|=0, \quad t \geq T
$$

for a fixed $T>0$. In addition, one can be shown that the feedback law $u_{h}$ is globally bounded:

$$
\sup _{x \in \mathbb{R}^{n}}\left|u_{h}(x)\right| \leq M<+\infty,
$$

where $M$ depends on $T$ as follows: smaller $T$ implies larger $M$. The homogeneous control stabilizes the considered system globally and in a finite time. It solves the stabilization problem considered above independently of $\varepsilon>0$. Due to the global boundedness of the controller it does not have the unbounded "peaking" effect discovered for the linear system as $\varepsilon \rightarrow 0$.

\subsubsection{Separation principle}

For a linear system, an output dynamic feedback can be constructed by performing independently any design of observer and controller gains, which is called the separation principle. Such a concept simplifies significantly the control development and its implementation, however, it is a well-known fact that the separation principle no longer holds 
for nonlinear dynamical systems. A reason for that is insensibility of the independently designed closed-loop system to the converging estimation error, which may create an unstable behavior. In some cases the quality can be seriously damaged by an asymptotically decaying estimator, but the performance can be recovered if the error converges exactly to zero after a finite interval of time, that can be interpreted as validity of the separation principle while using faster than asymptotically (exponentially) converging observers.

For an illustration consider a nonlinear system:

$$
\begin{aligned}
\dot{z} & =-a z+b x_{2} z(1+\ln |z|)+u, \\
\dot{x}_{1} & =-k_{1} x_{1}+x_{2}, \\
\dot{x}_{2} & =-k_{2} x_{1}+\sin (z),
\end{aligned}
$$

where $z, x_{1}, x_{2} \in \mathbb{R}$ are the states, $a, b, k_{1}, k_{2}$ are positive parameters, $u \in \mathbb{R}$ is the control input, and

$$
y_{1}=z, y_{2}=x_{1}
$$

are the outputs available for measurements.

It is easy to check that the state control

$$
u=-b x_{2} z(1+\ln |z|)
$$

stabilizes the system globally with an exponential rate of convergence.

Similarly, a conventional high-gain observer can be designed to ensure the estimation of the variable $x_{2}$ :

$$
\begin{aligned}
& \dot{\hat{x}}_{1}=-k_{1} \hat{x}_{1}+\hat{x}_{2}+\ell_{1}\left(y_{2}-\hat{x}_{1}\right), \\
& \dot{\hat{x}}_{2}=-k_{2} \hat{x}_{1}+\sin \left(y_{1}\right)+\ell_{2}\left(y_{2}-\hat{x}_{1}\right),
\end{aligned}
$$

where $\hat{x}_{1}, \hat{x}_{2} \in \mathbb{R}$ are the estimates, and $\ell_{1}, \ell_{2}$ are positive observer gains. Their tuning ensures a global exponential convergence of the estimation errors $x_{i}-\hat{x}_{i}, i=1,2$ with any desired decay: there exist $M>0$ and $\sigma>0$ such that $\left|x_{i}(t)-\hat{x}_{i}(t)\right| \leq M e^{-\sigma t} E, i=1,2$ for all $t \geq 0$, where $E=\sqrt{\left|x_{1}(0)-\hat{x}_{1}(0)\right|^{2}+\left|x_{2}(0)-\hat{x}_{2}(0)\right|^{2}}$ is an evaluation of the initial deviation.

Here both, the control and the observer, are selected independently, and substituting the obtained estimates in the control

$$
u=-b \hat{x}_{2} y_{1}\left(1+\ln \left|y_{1}\right|\right),
$$


we get the closed-loop dynamics for the variable $z$ (the dynamics of the variables $x_{1}, x_{2}$ are unchanged):

$$
\dot{z}=-a z+b\left(x_{2}-\hat{x}_{2}\right) z(1+\ln |z|) .
$$

To evaluate the behavior of $z$, consider a Lyapunov function $V(z)=|z|$, whose derivative admits an estimate:

$$
\dot{V} \leq-a V+\beta E e^{-\sigma t} V(1+\ln V)
$$

for $\beta=b M$, and introducing an auxiliary variable $v=\ln V$ we get:

$$
\dot{v} \leq-a+\beta E e^{-\sigma t}(1+v),
$$

whose solutions can be easily calculated analytically:

$$
v(t) \leq(v(0)+1) e^{\frac{\beta E}{\sigma}\left(1-e^{-\sigma t}\right)}-1-a \int_{0}^{t} e^{\frac{\beta E}{\sigma}\left(e^{-\sigma s}-e^{-\sigma t}\right)} d s,
$$

or, equivalently,

$$
|z(t)| \leq e^{(\ln |z(0)|+1) e^{\frac{\beta E}{\sigma}\left(1-e^{-\sigma t}\right)}-1-a \int_{0}^{t} e^{\frac{\beta E}{\sigma}\left(e^{-\sigma s}-e^{-\sigma t}\right)} d s .}
$$

Note that the integral $\int_{0}^{t} e^{\frac{\beta E}{\sigma}\left(e^{-\sigma s}-e^{-\sigma t}\right)} d s$ is a strictly growing unbounded positive function of time $t \geq 0$, and $(\ln |z(0)|+1) e^{\frac{\beta E}{\sigma}\left(1-e^{-\sigma t}\right)}-1$ is also an exponentially growing positive function, but asymptotically attaining its maximum $(\ln |z(0)|+1) e^{\frac{\beta E}{\sigma}}-1$. The latter value characterizes the peaking overshoot admissible for the variable $z$ and, for example, for $|z(0)| \leq 10, E \leq 10, b=M=\beta=1$ and $\sigma=5$, which are reasonable values of parameters, we get this value of order $10^{10}$.

Now assume that a fixed-time converging observer is designed (see Chapter 5 for the details) such that the estimation error admits a positive definite and radially unbounded Lyapunov function $W\left(x_{1}-\hat{x}_{1}, x_{2}-\hat{x}_{2}\right)$ yielding the following estimate on time derivative:

$$
\dot{W} \leq-\gamma\left(W^{1-\rho}+W^{1+\rho}\right),
$$

where $\gamma>0$ and $\rho \in(0,1]$ are design parameters, then

$$
W(t) \leq \tan ^{\rho^{-1}}\left(\arctan \left(W^{\rho}(0)\right)-\rho \gamma t\right)
$$


for $t \in\left[0, \frac{\arctan \left(W^{\rho}(0)\right)}{\rho \gamma}\right] \subseteq\left[0, \frac{\pi}{2 \rho \gamma}\right]$, and $W(t)=0$ afterwards. Hence, for the closed-loop system with such an observer, assuming that $\rho=1$ (without losing generality) the estimate for $V$ can be presented as

$$
\dot{V} \leq-a V+b \tan (\arctan (E)-\gamma t) V(1+\ln V)
$$

for $t \in\left[0, \frac{\arctan (E)}{\gamma}\right]$, and

$$
\dot{V} \leq-a V
$$

for $t \geq \frac{\arctan (E)}{\gamma}$. Obviously, we are interested only in the behavior on the initial interval of time, where using similar steps we get (note that $\int_{\tau}^{t} \tan (\arctan (E)-\gamma s) d s=\gamma^{-1} \ln \frac{|\cos (\arctan (E)-\gamma t)|}{|\cos (\arctan (E)-\gamma \tau)|}$ for any $\left.\tau \leq t\right)$ :

$$
\begin{aligned}
\ln |z(t)| \leq & \ln |z(0)| e^{\frac{b}{\gamma} \ln \frac{|\cos (\arctan (E)-\gamma t)|}{|\cos (\arctan (E))|}} \\
& +\int_{0}^{t} e^{\frac{b}{\gamma} \ln \frac{|\cos (\arctan (E)-\gamma t)|}{|\cos (\arctan (E)-\gamma \tau)|}(b \tan (\arctan (E)-\gamma t)-a) d \tau}
\end{aligned}
$$

for $t \in\left[0, \frac{\arctan (E)}{\gamma}\right]$. On this time interval $\cos (\arctan (E)-\gamma t)$ is a growing nonnegative function of time, hence, the same is $e^{\frac{b}{\gamma} \ln \frac{|\cos (\arctan (E)-\gamma t)|}{|\cos (\arctan (E))|}}$, but $b \tan (\arctan (E)-\gamma t)-a$ changes the sign from positive to the negative one, and all these functions are bounded for bounded $E$ (unbounded value is possible for $E=+\infty$ and $t=0$ only). In this case, again the peaking is governed by the first term $\ln |z(0)| e^{\frac{b}{\gamma} \ln \frac{|\cos (\arctan (E)-\gamma t)|}{|\cos (\arctan (E))|}}=$ $\left(\frac{|\cos (\arctan (E)-\gamma t)|}{|\cos (\arctan (E))|}\right)^{\frac{b}{\gamma}} \ln |z(0)| \leq|\cos (\arctan (E))|^{-\frac{b}{\gamma}} \ln |z(0)|$ (proportional to the initial conditions), which is much smaller than the value obtained for the linear high-gain observer. For example, for the same values of parameters in fixed-time scenario the maximum peaking is less than 52 , then by tuning the observer parameters it is possible to anticipate such a peaking, while in the case with a parasitic discrepancy of magnitude $10^{10}$ the gain adjustment cannot help.

\subsection{The structure of the paper}

After providing a short historical overview and several motivation examples, the paper has three main parts dealing with analysis, design and extensions, respectively. 
In the first part, the definitions of the different finite-/fixed-time stability properties are given together with their sufficient and necessary characterizations via the Lyapunov function approach. The problem of stabilization with accelerated rates is presented, and robust stability concepts (in the input-to-state stability sense) are described. Next, a detailed presentation of the theory of homogeneous systems is added, and the links with finite-/fixed-time convergence are shown.

In the second part, several stabilization algorithms for linear and nonlinear systems are formalized, which are based on the implicit Lyapunov function approach (an advantage of this method is that the tuning of control parameters can be performed by looking for solutions of linear matrix inequalities). These results are complemented by an observer with accelerated converge rate.

In the third part, the issues of discretization of finite-/fixed-time stable systems are discussed, with a special attention to the solutions obtained with the implicit Lyapunov function method. Finally, the accelerated converge concepts are presented for systems described by time-delay and partial differential equations.

The notation is summarized in the Appendix.

Remark 1.1. The sliding mode control and estimation algorithms are well-known for finite-time convergence behavior Filippov, 1988; Shtessel et al., 2014, but in this survey we will mainly focus our attention on continuous systems. 
Part I

Analysis 
This part has two chapters, 2 and 3 , representing the basics on two main approaches for investigation of finite-time stability in dynamical systems: the Lyapunov tools and the theory of homogeneity. We will focus on the models described by ordinary differential equations, and a more complex case will be shortly introduced at the end of this chapter, while time-delay systems and evolution equations will be considered with more details in the last part (Chapter 7).

The characterizations of the conventional global asymptotic stability property of the equilibrium at zero (see, e.g., Khalil, 2002) imply that

- to any compact neighborhood of the origin, the maximal time of settling in it is infinite for initial conditions belonging the complement of the neighborhood;

- from any compact neighborhood of the origin, the maximal time of convergence to the equilibrium is infinite.

Thus, the trajectories asymptotically (with the time approaching infinity) approach either a neighborhood of the origin or the origin itself from arbitrary initial conditions or ones on a sphere, respectively. And in this work, while speaking about finite-time convergence properties, we will include into consideration all decay characteristics when at least one of these convergence times (to a compact set from infinity, or to the origin from a sphere) is bounded. Such an attraction time can be dependent on initial conditions or uniform in the deviations of the state. 


\section{Notions of interest}

In this section, the formal definitions of different stability concepts with accelerated convergence rates will be given, and several necessary and sufficient conditions are discussed given in the Lyapunov function framework. For a wide class of dynamical systems, the existence of a Lyapunov function is proven to be equivalent to asymptotic stability Khalil, 2002, and as we will show, the same results can be obtained for the systems with stronger-than-asymptotic convergence rates, but under additional hypotheses. A reason for appearing these auxiliary restrictions comes from an important observation obtained in Bhat and Bernstein, 2000, that a finite-time stable system cannot be Lipschitz continuous at the origin, so a global uniqueness of solutions in the backward time may be lost. There are other aspects of regularity dealing with the settling-time function (the time of convergence of the trajectories to the equilibrium), which influence the Lyapunov conditions of finitetime or fixed-time stability. We will also present sufficient conditions for accelerated stabilization of continuous autonomous systems that are affine in the control given in terms of control Lyapunov functions. Finally, the extensions to input-to-state stability with finite/fixed-time convergence rates are explored. 


\subsection{Definitions of finite-time and fixed-time stability}

We will start with giving the formal definitions of different stability concepts studied in the sequel.

\subsubsection{Stability definitions}

Let the domain $\Gamma \subseteq \mathbb{R}^{n}$ be an open connected set containing the origin (then $\partial \Gamma$ denotes its boundary). Consider the following autonomous system

$$
\dot{x}(t)=f(x(t)), \quad x(t) \in \Gamma, t \geq 0,
$$

where $f \in C\left(\Gamma, \mathbb{R}^{n}\right)$ and $f(0)=0$. Let us assume that $f$ is such that (2.1) has the properties of existence and uniqueness of solutions in forward time at least locally outside the origin, then $x\left(t, x_{0}\right)$ denotes the solution to system (2.1) starting from $x_{0} \in \Gamma$ at $t=0$.

The notion of finite-time stability was introduced in the middle of the previous century (Zubov, 1964; Roxin, 1966). Here, following the results presented in (Zubov, 1964; Roxin, 1966; Khalil, 2002; Bhat and Bernstein, 2000; Cruz-Zavala et al., 2011; Polyakov, 2012; Song et al., 2017), we summarize the following list of stability properties, which are differing in the rates of convergence:

Definition 2.1. The origin of the system (2.1) is said to be

Lyapunov stable if for any $x_{0} \in \Gamma$ the solution $x\left(t, x_{0}\right)$ is defined for all $t \geq 0$, and for any $\epsilon>0$ there is $\delta>0$ such that for any $x_{0} \in \Gamma$, if $\left\|x_{0}\right\| \leq \delta$ then $\left\|x\left(t, x_{0}\right)\right\| \leq \epsilon$ for all $t \geq 0$;

asymptotically stable (AS) if it is Lyapunov stable and

$$
\lim _{t \rightarrow+\infty}\left\|x\left(t, x_{0}\right)\right\|=0
$$

for any $x_{0} \in \Gamma$;

finite-time stable (FTS) if it is Lyapunov stable and finite-time converging from $\Gamma$, i.e., for any $x_{0} \in \Gamma$ there exists $0 \leq T<+\infty$ such that $x\left(t, x_{0}\right)=0$ for all $t \geq T$. The function

$$
T\left(x_{0}\right)=\inf \left\{T \geq 0: x\left(t, x_{0}\right)=0 \forall t \geq T\right\}
$$


is called the settling-time function of the system (2.1);

fixed-time stable (FxTS) if it is finite-time stable and

$$
\sup _{x_{0} \in \Gamma} T\left(x_{0}\right)<+\infty ;
$$

prescribed-time stable (PTS) if it (a non-autonomous system, see Remark 2.1) is FxTS and $T\left(x_{0}\right)=$ const for all $x_{0} \in \Gamma \backslash\{0\}$; nearly fixed-time stable if it is Lyapunov stable and for any $\rho>0$ there exists $T_{\rho} \in(0,+\infty)$ such that $\left\|x\left(t, x_{0}\right)\right\| \leq \rho$ for all $t \geq T_{\rho}$ and all $x_{0} \in \Gamma$.

The set $\Gamma$ is called the domain of attraction. If $\Gamma=\mathbb{R}^{n}$, then the corresponding properties become global.

The characteristics introduced in Definition 2.1 for the system (2.1) constitute the main subject of interest for this survey. They admit the following relations:

\begin{tabular}{|cccccc|}
\hline PTS & $\subset$ & FxTS & $\subset$ & FTS \\
& & $\cap$ & & $\cap$ \\
& & nearly FxTS & $\subset$ & AS \\
& & & \\
\end{tabular}

It could be that $\sup _{0 \leq \rho \leq \rho_{0}} T_{\rho}=+\infty$ for any $\rho_{0}>0$ for a nearly FxTS system (2.1), which is the difference between nearly FxTS and FxTS properties $\left(\sup _{\rho \geq 0} T_{\rho}<+\infty\right.$ for the latter). Note also that for investigation of FxTS and nearly FxTS properties, $\Gamma=\mathbb{R}^{n}$ is the most interesting case study (it represents an unbounded set $\Gamma$, when the boundedness of $T$ or $T_{\rho}$ is an advantageous feature).

Example 2.1. Consider a scalar system:

$$
\dot{x}(t)=-(1-x(t)) x(t)
$$

with $x(t) \in \Gamma=(-1,1)$, which is asymptotically stable at the origin, but not nearly FxTS. In the same line, let

$$
\dot{x}(t)=-(1-x(t)) x^{\frac{1}{3}}(t),
$$

then at the origin for $\Gamma=(-1,1)$ this system is FTS, but not FxTS. 
Replacing in Definition 2.1 the distance to the origin $\|x\|$ with the distance $\|x\|_{\mathcal{A}}=\inf _{y \in \mathcal{A}}\|x-y\|$ to a compact set $\mathcal{A} \subset \Gamma$ it is possible to formulate the corresponding stability notions with respect to the set $\mathcal{A}$.

Remark 2.1. The PTS property is difficult to realize in (2.1) and it is usually provided for non-autonomous dynamical systems, as $\dot{x}(t)=$ $f\left(t, x(t)\right.$ ), for $t \geq 0$ and $x(t) \in \mathbb{R}^{n}$, (Song et al., 2017; Holloway and Krstic, 2019). It is also found advantageous for accelerated stabilization of distributed-parameter systems (Espitia et al., 2019; Steeves et al., 2020). A simplest example of a PTS scalar system is

$$
\dot{x}(t)=-\frac{x(t)}{T-t}
$$

with $t \geq 0, x_{0} \in \Gamma=\mathbb{R}$ and $T>0$ being the settling-time constant. It is easy to see that for any $x_{0} \in \Gamma$ the corresponding solution reaches the origin for $t=T$. Since in this part we mainly focus our attention on autonomous systems as in (2.1), in the sequel we will not consider the PTS property.

The next lemma states important properties of the settling-time function for an FTS system:

Lemma 2.1 (Bhat and Bernstein, 2000). Suppose that the origin of (2.1) is FTS on $\Gamma$ with the settling-time function $T: \Gamma \rightarrow \mathbb{R}_{+}$, then

(i) for any $x_{0} \in \Gamma$ and $t \in \mathbb{R}_{+}$:

$$
T\left(x\left(t, x_{0}\right)\right)=\max \left\{T\left(x_{0}\right)-t, 0\right\} .
$$

(ii) $T \in C\left(\Gamma, \mathbb{R}_{+}\right)$if and only if $T$ is continuous at 0 .

Since FxTS implies FTS, this lemma remains also valid for the fixed-time case. The result of Lemma 2.1 opens the door for application of $T$ as a Lyapunov function in analysis of FTS, and if $T \in C\left(\Gamma, \mathbb{R}_{+}\right)$, then

$$
\frac{\partial T\left(x\left(t, x_{0}\right)\right)}{\partial t}=-1
$$

for all $t \in\left[0, T\left(x_{0}\right)\right)$ and all $x_{0} \in \Gamma$.

Since by assumptions the system (2.1) admits unique solutions for all initial conditions from $\Gamma \backslash\{0\}$, the solutions in backward time may also exist: 
Definition 2.2. The origin of the system (2.1) is called complete (nearly) FxTS, if it is (nearly) FxTS and for any $x_{0} \in \Gamma \backslash\{0\}$ there exists a unique solution in the backward time denoted as $x\left(-t, x_{0}\right)$ for $t \in\left[0, \tau\left(x_{0}\right)\right)$, where $\tau: \Gamma \backslash\{0\} \rightarrow \mathbb{R}_{+}$is the escape-time function such that for all $x_{0} \in \Gamma \backslash\{0\}$

$$
\limsup _{t \rightarrow \tau\left(x_{0}\right)}\left\|x\left(-t, x_{0}\right)\right\|_{\delta \Gamma}=0 \quad \text { or } \quad \limsup _{t \rightarrow \tau\left(x_{0}\right)}\left\|x\left(-t, x_{0}\right)\right\|=+\infty .
$$

The infinite limit is possible if $\Gamma$ is unbounded.

Note that by this definition, if $T\left(x_{0}\right) \leq T_{\max }$ for all $x_{0} \in \Gamma$, then $\tau\left(x_{0}\right) \leq T_{\max }$ also for all $x_{0} \in \Gamma \backslash\{0\}$, since

$$
T\left(x\left(t, x_{0}\right)\right)+\tau\left(x\left(t, x_{0}\right)\right)=\text { const }
$$

for all $t \geq 0$ such that $\left\|x\left(t, x_{0}\right)\right\| \notin\{0,+\infty\}$. Therefore, by Lemma 2.1:

$$
\tau\left(x\left(-t, x_{0}\right)\right)=\max \left\{0, \tau\left(x_{0}\right)-t\right\} .
$$

Hence, provided that $\tau \in C\left(\Gamma \backslash\{0\}, \mathbb{R}_{+}\right)$,

$$
\frac{\partial \tau\left(x\left(-t, x_{0}\right)\right)}{\partial t}=-1
$$

for all $t \in\left[0, \tau\left(x_{0}\right)\right)$ and all $x_{0} \in \Gamma \backslash\{0\}$, similarly to $T$.

\subsubsection{Lyapunov conditions}

For $V \in C(\Gamma, \mathbb{R})$, the upper-right Dini derivative along the solutions of (2.1) is given by

$$
\dot{V}\left(x_{0}\right)=D^{+}(V \circ x)\left(0, x_{0}\right)
$$

for any $x_{0} \in \Gamma$, where

$$
D^{+} g(0)=\limsup _{h \rightarrow 0^{+}} \frac{1}{h}[g(h)-g(0)]
$$

is the upper-right Dini derivative of a function $g \in C\left(\mathbb{R}_{+}, \mathbb{R}\right)$, if additionally $V \in C^{1}(\Gamma \backslash\{0\}, \mathbb{R})$, then

$$
\dot{V}(x)=\frac{\partial V(x)}{\partial x} f(x), \quad \forall x \in \Gamma \backslash\{0\} .
$$


Definition 2.3 (Krasovskii, 1963). For the system (2.1), $V \in$ $C\left(\Gamma, \mathbb{R}_{+}\right)$is called a strict Lyapunov function (LF) if it fulfills the following properties:

1) Positive definiteness: $V(x)>0$ for all $x \in \Gamma \backslash\{0\}$ and $V(0)=0$.

2) Radial unboundedness on $\Gamma: V(x) \rightarrow+\infty$ as $x \rightarrow \partial \Gamma$, and if $\Gamma$ is unbounded then, in addition, $V(x) \rightarrow+\infty$ as $\|x\| \rightarrow+\infty$.

3) Strict decrement on the trajectories of $(2.1): \dot{V}(x)<0$ for all $x \in \Gamma \backslash\{0\}$.

It is well known that existence of a strict LF is necessary and sufficient for AS of the origin for (2.1):

Theorem 2.2 (Krasovskii, 1963). The origin of (2.1) is AS on $\Gamma$ if and only if there exists a strict LF for (2.1).

Asymptotic stability includes all other properties in Definition 2.1, and it does not present a restriction on the rate of decreasing of a LF. Thus, the equivalent formulation of AS in terms of existence of a LF is the shortest one. For other types of convergence the conditions are more demanding.

\section{The equivalent conditions explicitly dependent on $\dot{V}$}

For the system (2.1), for any $x_{0} \in \Gamma$ and $t \geq 0$ define the map $\theta_{x_{0}}$ : $s \in \mathbb{R}_{+} \rightarrow t \in \mathbb{R}_{+}$by the identity $s=V\left(x\left(\theta_{x_{0}}(s), x_{0}\right)\right)$, which is welldefine since $V\left(x\left(t, x_{0}\right)\right)$ is a strictly decreasing function of time (it is parameterized by the initial condition $x_{0}$ ).

The following theorem gives necessary and sufficient conditions of various finite-time stability properties for an autonomous system:

Theorem 2.3. The following properties are equivalent for the system (2.1):

1) The origin is FTS on $\Gamma \Leftrightarrow$ There exists a strict LF $V \in$ 
$C^{\infty}\left(\Gamma, \mathbb{R}_{+}\right)$satisfying for all $x_{0} \in \Gamma$

$$
\int_{V\left(x_{0}\right)}^{0} \frac{d s}{\dot{V}\left(x\left(\theta_{x_{0}}(s), x_{0}\right)\right)}<+\infty .
$$

2) The origin is FxTS on $\Gamma \Leftrightarrow$ There exists a strict LF $V \in$ $C\left(\Gamma, \mathbb{R}_{+}\right)$satisfying for all $x_{0} \in \Gamma$

$$
\sup _{x_{0} \in \Gamma} \int_{V\left(x_{0}\right)}^{0} \frac{d s}{\dot{V}\left(x\left(\theta_{x_{0}}(s), x_{0}\right)\right)}<+\infty .
$$

3) The origin is nearly FxTS on $\Gamma \Leftrightarrow$ There exists a strict LF $V \in C\left(\Gamma, \mathbb{R}_{+}\right)$satisfying for all $x_{0} \in \Gamma$ and $\rho>0$

$$
\sup _{x_{0} \in \Gamma} \int_{V\left(x_{0}\right)}^{\rho} \frac{d s}{\dot{V}\left(x\left(\theta_{x_{0}}(s), x_{0}\right)\right)}<+\infty .
$$

Proof. The part 1) was proven in (Moulay and Perruquetti, 2006). The part 2) was given in (Lopez-Ramirez et al., 2019).

For the part 3), if the system (2.1) is nearly FxTS, then a vicinity $B\left(\rho^{\prime}\right)$ of the origin for any $\rho^{\prime}>0$ is reached in a finite time for all $x_{0} \in \Gamma$. Hence, nearly FxTS implies AS by continuity and Lyapunov stability of (2.1). According to Theorem 2.2, there exists a strict Lyapunov function $V$ for (2.1) and, therefore, there exists a well-defined mapping $\left[0, T\left(x_{0}\right)\right) \rightarrow\left(0, V\left(x_{0}\right)\right], t \mapsto V\left(x\left(t, x_{0}\right)\right)$ strictly decreasing and differentiable for all $t \in\left[0, T\left(x_{0}\right)\right)$. Hence, for any $x_{0} \in \Gamma$, there exists a differentiable inverse mapping $\left(0, V\left(x_{0}\right)\right] \rightarrow\left[0, T\left(x_{0}\right)\right)$ denoted above as $s \stackrel{\theta_{x}}{\mapsto} t$, also decreasing that satisfies for all $s \in\left(0, V\left(x_{0}\right)\right]$

$$
\theta_{x_{0}}^{\prime}(s)=\frac{1}{\dot{V}\left(x\left(\theta_{x_{0}}(s), x_{0}\right)\right)} .
$$

For any $\rho>0$ and $x_{0} \in \Gamma$ denote $T_{\rho}\left(x_{0}\right)=\inf \left\{T \geq 0: V\left(x\left(t, x_{0}\right)\right) \leq\right.$ $\rho \forall t \geq T\}$ as the time of convergence of the trajectory $x\left(t, x_{0}\right)$ to a neighborhood of the origin where $V(x) \leq \rho$ (by radial unboundedness of $V$, it is included in $B\left(\rho^{\prime}\right)$ for some $\left.\rho^{\prime}\right)$. Then the change of variables $s=V\left(x\left(t, x_{0}\right)\right)$ and the fact that $V\left(x\left(T_{\rho}\left(x_{0}\right), x_{0}\right)\right)=\rho$ for all $x_{0} \in \Gamma$ lead to

$$
T_{\rho}\left(x_{0}\right)=\int_{0}^{T_{\rho}\left(x_{0}\right)} d t=\int_{V\left(x_{0}\right)}^{\rho} \theta_{x_{0}}^{\prime}(s) d s=\int_{V\left(x_{0}\right)}^{\rho} \frac{d s}{\dot{V}\left(\Phi\left(\theta_{x_{0}}(s), x_{0}\right)\right)} .
$$


Then we have that

$$
+\infty>\sup _{x_{0} \in \Gamma} T_{\rho}\left(x_{0}\right)=\sup _{x_{0} \in \Gamma} \int_{V\left(x_{0}\right)}^{\rho} \frac{d s}{\dot{V}\left(\Phi\left(\theta_{x_{0}}(s), x_{0}\right)\right)}
$$

and the conclusion readily follows.

Inversely, according to Theorem 2.2, existence of a strict Lyapunov function $V$ for the system (2.1) guarantees that the origin is AS, and therefore, the mapping $\theta_{x_{0}}$ is well-defined. The equation (2.6) implies, furthermore, that the origin is nearly FxTS.

Remark 2.2. Moreover if 1) or 2) are verified, all strict LFs $V \in$ $C^{\infty}\left(\Gamma, \mathbb{R}_{+}\right)$for $(2.1)$ satisfy for all $x_{0} \in \Gamma$

$$
T\left(x_{0}\right)=\int_{V\left(x_{0}\right)}^{0} \frac{d s}{\dot{V}\left(x\left(\theta_{x_{0}}(s), x_{0}\right)\right)}<+\infty .
$$

The conditions (2.3), (2.4) and (2.5) are in general difficult to verify, since they require an explicit knowledge of the system's trajectories. However, they can be used to discard the kinds of stability for certain systems. The next examples, partly borrowed from (Moulay and Perruquetti, 2006), demonstrate this ability:

Example 2.2. Consider a scalar linear system

$$
\dot{x}(t)=-x(t),
$$

$x_{0} \in \Gamma=\mathbb{R}$, and the Lyapunov function candidate $V(x)=x^{2}$. Then $x\left(t, x_{0}\right)=e^{-t} x_{0}, V\left(x\left(t, x_{0}\right)\right)=e^{-2 t} x_{0}^{2}, \theta_{x_{0}}(s)=\ln \left(x_{0}\right)-\frac{1}{2} \ln (s)$ and $x\left(\theta_{x_{0}}(s), x_{0}\right)=\sqrt{s}$, where $s>0$, which leads to $\dot{V}\left(x\left(\theta_{x_{0}}(s), x_{0}\right)\right)=-2 s$. Substituting this expression in (2.7) yields

$$
T\left(x_{0}\right)=\int_{V\left(x_{0}\right)}^{0} \frac{d s}{\dot{V}\left(x\left(\theta_{x_{0}}(s), x_{0}\right)\right)}=+\infty,
$$

and according to Theorem 2.3, the system (2.8) is not FTS.

Example 2.3. Similarly, for a scalar system

$$
\dot{x}(t)=-\lfloor x(t)\rceil^{1-\nu}
$$


with $\nu \in(0,1)\left(\left\lfloor\left.\cdot\right|^{\alpha}=|\cdot|{ }^{\alpha} \operatorname{sign}(\cdot)\right.\right.$ for any $\left.\alpha \geq 0\right)$, which for any $x_{0} \in \Gamma=\mathbb{R}$ admits the solutions

$$
x\left(t, x_{0}\right)= \begin{cases}\left(\left|x_{0}\right|^{\nu}-\nu t\right)^{\nu^{-1}} \operatorname{sign}\left(x_{0}\right) & \text { if } t \in\left[0, T\left(x_{0}\right)\right) \\ 0 & \text { if } t \geq T\left(x_{0}\right)\end{cases}
$$

where the settling-time function

$$
T\left(x_{0}\right)=\frac{\left|x_{0}\right|^{\nu}}{\nu}
$$

is continuous, consider a LF candidate $V(x)=|x|^{\nu}$, which yields $V\left(x\left(t, x_{0}\right)\right)=\left|x_{0}\right|^{\nu}-\nu t$ for $t \in\left[0, T\left(x_{0}\right)\right), \theta_{x_{0}}(s)=\frac{\left|x_{0}\right|^{\nu}-s}{\nu}$ and $x\left(\theta_{x_{0}}(s), x_{0}\right)=$ $s^{\nu^{-1}} \operatorname{sign}\left(x_{0}\right)$ for $s>0$, then $\dot{V}\left(x\left(\theta_{x_{0}}(s), x_{0}\right)\right)=-\nu$. Therefore,

$$
\int_{V\left(x_{0}\right)}^{0} \frac{d s}{\dot{V}\left(\Phi\left(\theta_{x_{0}}(s), x_{0}\right)\right)}=\frac{1}{\nu} \int_{0}^{\left|x_{0}\right|^{\nu}} d s=\frac{1}{\nu}\left|x_{0}\right|^{\nu}
$$

and it becomes clear that the property (2.3) is satisfied while (2.4) is not. Hence, the system (2.9) is FTS, but not FxTS.

Example 2.4. Finally, for the system

$$
\dot{x}(t)=-\lfloor x(t)\rceil^{0.5}-\lfloor x(t)\rceil^{1.5}
$$

with the solution

$$
x\left(t, x_{0}\right)=\left\{\begin{array}{ll}
\tan \left[\arctan \left(\left|x_{0}\right|^{\frac{1}{2}}\right)-\frac{1}{2} t\right]^{2} \operatorname{sign}\left(x_{0}\right) & \text { if } 0 \leq t \leq T\left(x_{0}\right) \\
0 & \text { if } t>T\left(x_{0}\right)
\end{array},\right.
$$

also defined for any $x_{0} \in \Gamma=\mathbb{R}$, where the settling-time function $T\left(x_{0}\right)=2 \arctan \left(\left|x_{0}\right|^{\frac{1}{2}}\right)$, consider a LF candidate $V(x)=|x|$, which leads to $V\left(x\left(t, x_{0}\right)\right)=\tan \left[\arctan \left(\left|x_{0}\right|^{\frac{1}{2}}\right)-\frac{1}{2} t\right]^{2}$ for $t \in\left[0, T\left(x_{0}\right)\right), \theta_{x_{0}}(s)=$ $2\left(\arctan \left(\left|x_{0}\right|^{\frac{1}{2}}\right)-\arctan (\sqrt{s})\right)$ and $x\left(\theta_{x_{0}}(s), x_{0}\right)=s \operatorname{sign}\left(x_{0}\right)$ for $s>0$, then $\dot{V}\left(x\left(\theta_{x_{0}}(s), x_{0}\right)\right)=-s^{0.5}-s^{1.5}$ and

$$
\begin{aligned}
\sup _{x_{0} \in \Gamma} \int_{V\left(x_{0}\right)}^{0} & \frac{d s}{\dot{V}\left(\Phi\left(\theta_{x_{0}}(s), x_{0}\right)\right)}=\sup _{x_{0} \in \Gamma} \int_{0}^{\left|x_{0}\right|} \frac{d s}{s^{0.5}+s^{1.5}} \\
& =\sup _{x_{0} \in \Gamma} 2 \arctan \left(\left|x_{0}\right|^{0.5}\right) \leq \pi .
\end{aligned}
$$




\section{Sufficient conditions dependent of an upper estimate on $\dot{V}$}

In the previous subsection the properties of the function $\dot{V}(x)$ were used directly. However, usually, a sufficient condition for AS in terms of existence of a LF is formulated as follows: the system (2.1) admits a strict LF $V \in C\left(\Gamma, \mathbb{R}_{+}\right)$(for instance, if $\Gamma=\mathbb{R}^{n}, \alpha_{1}(\|x\|) \leq V(t, \phi) \leq \alpha_{2}(\|x\|)$ for some functions $\left.\alpha_{1}, \alpha_{2} \in \mathcal{K}_{\infty}\right)$ with $\alpha_{3} \in \mathcal{K}$ such that

$$
\dot{V}(x) \leq-\alpha_{3}(V(x))
$$

for all $x \in \Gamma$, then the properties of the function $\alpha_{3}$ from an upper estimate of $\dot{V}(x)$ can be related with different rates of convergence as in the next result:

Theorem 2.4. If the system (2.1) possesses a strict LF $V \in$ $C\left(\Gamma, \mathbb{R}_{+}\right)$satisfying $(2.13)$ for some $\alpha_{3} \in \mathcal{K}$, then the origin is

- FTS with a continuous settling-time function $T(x) \leq$ $\int_{0}^{V(x)} \frac{d z}{\alpha_{3}(z)}$ provided that $\int_{0}^{\epsilon} \frac{d z}{\alpha_{3}(z)}<+\infty$ for some $\epsilon>0$;

- nearly FxTS provided that $\int_{\epsilon}^{\sup _{x \in \Gamma} V(x)} \frac{d z}{\alpha_{3}(z)}<+\infty$ for some $\epsilon>0$

- FxTS with a continuous settling-time function $T(x) \leq$ $\int_{0}^{\sup _{x \in \Gamma} V(x)} \frac{d z}{\alpha_{3}(z)}$ provided that $\int_{0}^{\sup _{x \in \Gamma} V(x)} \frac{d z}{\alpha_{3}(z)}<+\infty$.

Proof. Since all conditions of Theorem 2.2 are satisfied, the system (2.1) is AS. The FTS and FxTS parts have been proven in (Moulay and Perruquetti, 2006) and (Lopez-Ramirez et al., 2019), respectively.

The nearly FxTS part can be proven using similar arguments: since $\frac{d V\left(x\left(t, x_{0}\right)\right)}{d t} \leq \dot{V}\left(x_{0}\right)$ for any $x_{0} \in \Gamma$, we get

$$
\frac{d V}{\alpha_{3}(V)} \leq-d t
$$

whose integration by parts implies

$$
\int_{V\left(x_{0}\right)}^{V\left(x\left(t, x_{0}\right)\right)} \frac{d z}{\alpha_{3}(z)} \leq-t
$$


for all $x_{0} \in \Gamma$. Since the system is AS, for a given $\epsilon>0$ there exists $t_{\epsilon} \geq 0$ such that $V\left(x\left(t, x_{0}\right)\right) \leq \epsilon$ for all $t \geq t_{\epsilon}$, then for any $x_{0} \in \Gamma$ we obtain:

$$
t_{\epsilon} \leq \int_{\epsilon}^{V\left(x_{0}\right)} \frac{d z}{\alpha_{3}(z)} \leq \int_{\epsilon}^{\sup _{x_{0} \in \Gamma} V\left(x_{0}\right)} \frac{d z}{\alpha_{3}(z)}<+\infty,
$$

and the time $t_{\epsilon}$ is globally bounded, which for a AS system implies nearly FxTS property (for any $\rho>0$ such that the ball $B(\rho)$ contains the domain where $V(x) \leq \epsilon$, the time needed for a trajectory to reach $B(\rho)$ is finite; while for any such a ball $B(\rho)$ contained into interior of the compact set $V(x) \leq \epsilon$, the time of convergence from the level $\epsilon$ to the ball is finite due to AS).

A canonical selection for such a function $\alpha_{3}$ is $\alpha_{3}(s)=c s^{\alpha}$ for some $c>0$ and $\alpha \in[0,1)$ or $\alpha>1$ for FTS or nearly FxTS, respectively; for FxTS case $\alpha_{3}(s)=c_{1} s^{\alpha}+c_{2} s^{\beta}$ with $c_{1}, c_{2}>0, \alpha \in[0,1)$ and $\beta>1$ (combination of FTS and nearly FxTS cases) (Moulay and Perruquetti, 2006; Polyakov, 2012).

\section{Equivalent conditions for the case of a continuous settling-time func- tion}

It is worth highlighting that the restrictions of Theorem 2.4 imply continuity of the settling-time function $T$ in FTS and FxTS cases, but as we will show in the next result, under such a continuity of $T$ (and some additional mild conditions) the existence of a LF $V$ with $\alpha_{3}$ in the canonical form is necessary and sufficient for (2.1) (see (Bhat and Bernstein, 2000; Moulay and Perruquetti, 2006; Lopez-Ramirez et al., 2019)):

Theorem 2.5. The following properties are equivalent for the system (2.1):

1) the origin is FTS on $\Gamma$ with a continuous settling-time function $T \Leftrightarrow$ there exist $c>0, \alpha \in(0,1)$ and a strict $\operatorname{LF} V \in C\left(\Gamma, \mathbb{R}_{+}\right)$ satisfying for all $x \in \Gamma$ :

$$
\dot{V}(x) \leq-c V(x)^{\alpha} ;
$$


2) the origin is complete FxTS on $\Gamma$ with continuous settlingtime $T$ and escape-time $\tau$ functions $\Leftrightarrow$ there exist $c_{1}>0, c_{2}>0$, $\alpha>0, \beta>0$ and a strict $\mathrm{LF} V \in C\left(\Gamma, \mathbb{R}_{+}\right)$satisfying for all $x \in \Gamma \backslash\{0\}:$

$$
\dot{V}(x) \leq-c_{1} V^{\frac{\alpha}{\alpha+1}}(x)-c_{2} V^{\frac{\beta+1}{\beta}}(x) ;
$$

3) the origin is complete nearly FxTS on $\Gamma$ with continuous escape-time function $\tau \Leftrightarrow$ there exist $c>0, \alpha>1$ and a strict $\mathrm{LF}$ $V \in C\left(\Gamma, \mathbb{R}_{+}\right)$satisfying for all $x \in \Gamma \backslash\{0\}$ :

$$
\dot{V}(x) \leq-c V^{\alpha}(x) .
$$

Proof. The parts 1) and 2) have been proven in (Bhat and Bernstein, 2000) and (Lopez-Ramirez et al., 2019), respectively.

To prove assertion 3) assume that the system is complete nearly FxTS at the origin on $\Gamma$. Since any nearly FxTS system is globally asymptotically stable at $x=0$, there is a strict $\mathrm{LF} W \in C\left(\Gamma, \mathbb{R}_{+}\right)$ satisfying for all $x \in \Gamma \backslash\{0\}$ :

$$
\dot{W}(x) \leq-\eta(W(x))
$$

for some $\eta \in \mathcal{K}$ (see, for example, Corollary 1 in (Lopez-Ramirez et al., 2019)). Define two positive constants, $r$ and $R$, such that $0<2 r<R$, and consider the system (2.1) in the backward time:

$$
\dot{x}^{*}(t)=-f\left(x^{*}(t)\right), t \geq 0,
$$

then for any $x_{0} \in \Gamma \backslash\{0\}$ there is a unique solution $x\left(-t, x_{0}\right)=x^{*}\left(t, x_{0}\right)$ (to denote a solution of this system we use the notation for the solutions of (2.1) with negative time argument since we assumed that it exists due to complete nearly FxTS of (2.1)) that is defined on some finite interval of time for $t \in\left[0, \tau\left(x_{0}\right)\right)$, and

$$
\lim _{t \rightarrow \tau\left(x_{0}\right)}\left\|x\left(-t, x_{0}\right)\right\|_{\delta \Gamma}=0 \text { or } \lim _{t \rightarrow \tau\left(x_{0}\right)}\left\|x\left(-t, x_{0}\right)\right\|=+\infty .
$$

Recall that

$$
\tau\left(x\left(-t, x_{0}\right)\right)=\max \left\{0, \tau\left(x_{0}\right)-t\right\},
$$

then for a continuous function $\tau$ :

$$
\frac{\partial \tau\left(x\left(-t, x_{0}\right)\right)}{\partial t}=-1
$$


for all $t \in\left[0, \tau\left(x_{0}\right)\right)$ and all $x_{0} \in \Gamma \backslash\{0\}$. For

$$
x \in \Omega_{r}=\{x \in \Gamma: W(x) \geq r\}
$$

(this set is not empty since $W$ is radially unbounded on $\Gamma$ ) introduce a candidate LF function

$$
V_{1}(x)=\rho \tau(x)^{\frac{1}{1-\alpha}}
$$

for some $\rho>0$ specified later. The function $V_{1}$ is also radially unbounded on $\Gamma$ due to the properties of $\tau$ and since $\alpha>1$, its time derivative computed along trajectories of (2.1) can be rewritten as follows:

$$
\dot{V}_{1}(x)=-\frac{\rho}{\alpha-1} \tau(x)^{\frac{\alpha}{1-\alpha}}=-\frac{\rho^{1-\alpha}}{\alpha-1} V_{1}^{\alpha}(x)
$$

for all $x \in \Omega_{r}$.

Moreover, consider the related to the dynamics of $W$ a scalar comparison system in the backward time:

$$
\dot{y}(t)=\eta(y(t)), \quad t \in\left[0, \tau_{\eta}(y(0))\right]
$$

with initial conditions $y(0) \in(0, R]$ the escape-time function $\tau_{\eta}$ : $(0, R] \rightarrow \mathbb{R}_{+}$to the level $y=R$ is defined as follows

$$
\tau_{\eta}(y(0))=t^{*}>0: y\left(t^{*}\right)=R .
$$

Then, by construction,

$$
\tau_{\eta}(y)=\int_{y}^{R} \frac{d s}{\eta(s)}, \quad \frac{d \tau_{\eta}(y)}{d y}=\frac{-1}{\eta(y)}<0
$$

for all $y \in(0, R]$. The latter meant $\tau_{\eta}$ is a monotone function and

$$
\lim _{y \rightarrow 0} \frac{1}{\tau_{\eta}(y)}=\lim _{y \rightarrow 0} \frac{1}{\int_{y}^{R} \frac{d s}{\eta(s)}}=b \in[0,+\infty) .
$$

Repeating the same argumentation as for the function $\tau$ we derive

$$
\frac{\partial \tau_{\eta}(y(t))}{\partial t}=\left.\frac{d \tau_{\eta}(y)}{d y}\right|_{y=y(t)} \dot{y}(t)=-1
$$

for all $t \in\left[0, \tau_{\eta}\left(y_{0}\right)\right]$. Returning to the dynamics of $W$, if $W(t)=y(t)$, while $\dot{W}(t) \leq-\dot{y}(t)$ by design, we get

$$
\left.\frac{d \tau_{\eta}(y)}{d y}\right|_{y=W(t)} \dot{W}(t) \geq 1
$$


for all instants of time such that $W(t) \in(0, R]$. Define a local Lyapunov function candidate

$$
V_{2}(x)=\left(\frac{1}{\tau_{\eta}(W(x))}-b\right)^{\frac{1}{\alpha-1}}
$$

for $W(x) \leq R$, then

$$
\frac{\partial V_{2}(x)}{\partial x} f(x) \leq-\frac{1}{\alpha-1}\left(\frac{1}{\tau_{\eta}(W(x))}-b\right)^{\frac{\alpha}{\alpha-1}}=-\frac{1}{\alpha-1} V_{2}^{\alpha}(x)
$$

for $0<W(x) \leq R$.

Finally, let us unite the LF candidates $V_{1}$ and $V_{2}$ to obtain a strict LF $V \in C\left(\Gamma, \mathbb{R}_{+}\right)$as

$$
V(x)= \begin{cases}V_{2}(x) & \text { if } W(x) \leq r, \\ V_{1}(x) & \text { if } W(x) \geq 2 r, \\ \lambda(x) V_{1}(x)+(1-\lambda(x)) V_{2}(x) & \text { otherwise, }\end{cases}
$$

where $\lambda(x)=r^{-1}(W(x)-r)$ with $\dot{\lambda}(t) \leq-r^{-1} \eta(W(x))<0$, and $\rho$ is chosen in a way to guarantee that $V_{1}(x)>V_{2}(x)$ for all $x \in \Omega_{2 r} \backslash \Omega_{r}$. Such a design of $V$ ensures all the requirements imposed on a strict LF due to the proven properties of $V_{1}, V_{2}$, and since

$$
\dot{V}(x) \leq-\frac{1}{\alpha-1} \begin{cases}V_{2}^{\alpha}(x) & \text { if } W(x) \leq r, \\ \rho^{1-\alpha} V_{1}^{\alpha}(x) & \text { if } W(x) \geq 2 r, \\ \rho^{1-\alpha} \lambda(x) V_{1}^{\alpha}(x)+(1-\lambda(x)) V_{2}^{\alpha}(x) & \text { otherwise. }\end{cases}
$$

Hence, there exists $c>0$ such that

$$
\dot{V}(x) \leq-c V^{\alpha}(x)
$$

for all $x \in \Gamma \backslash\{0\}$ as required.

Now assume that the last inequality is satisfied, and let us show that the system (2.1) is complete nearly fixed-time stable. Obviously, it is nearly FxTS. Consider the system behavior in the backward time, then we obtain that

$$
-\frac{\partial V(x)}{\partial x} f(x) \geq c V^{\alpha}(x)
$$

from which we conclude that the escape-time function is upper bounded by a continuous function, and hence it is also continuous on $\Gamma$. 
Example 2.5. To illustrate this result, for the FTS and FxTS cases we can consider the examples (2.9) and (2.11) with the corresponding strict LF $V(x)=x^{2}$, then

$$
\dot{V}(x) \leq-2 V(x)^{1-\frac{\nu}{2}}
$$

or

$$
\dot{V}(x) \leq-2 V(x)^{0.75}-2 V(x)^{1.25}
$$

for all $x \in \Gamma$, respectively. The simplest scalar example for the nearly FxTS case is

$$
\dot{x}(t)=-\lfloor x(t)\rceil^{\mu}
$$

with $\mu>1$ and $\Gamma=\mathbb{R}$, then following Theorem 2.5 for $\operatorname{LF} V(x)=x^{2}$ :

$$
\dot{V}(x) \leq-2 V(x)^{\frac{1+\mu}{2}} .
$$

Example 2.6. To highlight complexity of the problem for the case of nearly FxTS, consider another scalar dynamics:

$$
\dot{x}(t)=\phi(x(t)), \phi(x)=\left\{\begin{array}{ll}
-x^{2} & \text { if } x>1 \\
-x^{x^{-1}} & \text { if } x \in[0,1] \\
\sqrt{2|x|} & \text { if } x \in[-2,0) \\
\frac{1}{2} x^{2} & \text { if } x<-2
\end{array},\right.
$$

where $\phi \in C(\mathbb{R}, \mathbb{R})$ and $\Gamma=\mathbb{R}$. It is not straightforward to check using, e.g., a standard for scalar systems $\operatorname{LF} W(x)=x^{2}$, that close to the origin this system admits a strict LF having the estimate $\dot{W}(x) \leq-c W^{\alpha}(x)$ for some $c>0$ and $\alpha \in \mathbb{R}_{+}$. However, the system is nearly FxTS due to the quadratic term appearing in $\phi$ for $x<-2$ or $x>1$, and since $|x|^{|x|^{-1}} \geq \rho^{\rho^{-1}}$ for all $|x| \in[\rho, 2]$ for any $\rho \in(0,2)$. In addition, due to asymmetric definition of $\phi$, the escape-time function $\tau$ takes different values for negative and positive argument, hence, $\tau^{-1}$ is discontinuous at zero. However, observing that

$$
\dot{W}(x) \leq-\eta(W(x)), \eta(s)=s \begin{cases}s^{s^{-1}} & s \leq 1 \\ s^{2} & s>1\end{cases}
$$

allows a needed escape-time function close to the origin to be derived (as in the proof of Theorem 2.5). 


\subsection{Robust stability in the presence of disturbances}

Consider an extension of the nonlinear system (2.1) for $\Gamma=\mathbb{R}^{n}$ with external inputs:

$$
\dot{x}(t)=f(x(t), d(t)), t \geq 0,
$$

where $x(t) \in \mathbb{R}^{n}$ is the state, $d(t) \in \mathbb{R}^{m}$ is the input, $d \in L^{\infty}\left(\mathbb{R}_{+}, \mathbb{R}^{m}\right)$; $f \in C\left(\mathbb{R}^{n+m}, \mathbb{R}^{n}\right)$ ensures forward existence of the system solutions, at least locally, and $f(0,0)=0$. For an initial condition $x_{0} \in \mathbb{R}^{n}$ and an input $d \in L^{\infty}\left(\mathbb{R}_{+}, \mathbb{R}^{m}\right)$, define the corresponding solution of (2.14) by $x\left(t, x_{0}, d\right)$ for any $t \geq 0$ for which the solution exists. Since (2.14) might not have unique solutions and we are interested in the strong stability notions only (satisfying for all solutions), then with a slight inexactness in the notation we will assume that if a property is satisfied for all initial conditions in a set, then it implies that it also holds for all solutions issued from those initial conditions.

\subsubsection{Definitions of robust accelerated stability}

The following two concepts represent the core properties of input-tostate stability theory (Sontag, 2007; Dashkovskiy et al., 2011):

Definition 2.4. The system (2.14) is called input-to-state stable (ISS) if there exist some functions $\beta \in \mathcal{K} \mathcal{L}$ and $\vartheta \in \mathcal{K}$ such that

$$
\left\|x\left(t, x_{0}, d\right)\right\| \leq \beta\left(\left\|x_{0}\right\|, t\right)+\vartheta\left(\|d\|_{(0, t)}\right) \quad \forall t \geq 0
$$

for any input $d \in L^{\infty}\left(\mathbb{R}_{+}, \mathbb{R}^{m}\right)$ and any $x_{0} \in \mathbb{R}^{n}$. The function $\vartheta$ is called the asymptotic gain.

Definition 2.5. The system (2.14) is called integral input-to-state stable (iISS) if there exist some functions $\beta \in \mathcal{K} \mathcal{L}, \alpha \in \mathcal{K}_{\infty}$ and $\vartheta \in \mathcal{K}$ such that

$$
\alpha\left(\left\|x\left(t, x_{0}, d\right)\right\|\right) \leq \beta\left(\left\|x_{0}\right\|, t\right)+\int_{0}^{t} \vartheta(\|d(s)\|) d s \quad \forall t \geq 0
$$

for any input $d \in L^{\infty}\left(\mathbb{R}_{+}, \mathbb{R}^{m}\right)$ and any $x_{0} \in \mathbb{R}^{n}$.

In order to define the property of accelerated ISS or iISS, conventional class- $\mathcal{K} \mathcal{L}$ functions are no longer suitable (Hong et al., 2010), 
therefore, generalizations of these functions are usually utilized (Hong et al., 2010; Lopez-Ramirez et al., 2020), as the following one:

Definition 2.6 (Lopez-Ramirez et al., 2020). A continuous function $\beta: \mathbb{R}_{+} \times \mathbb{R}_{+} \rightarrow \mathbb{R}_{+}$is a generalized class- $\mathcal{K} \mathcal{L}$ function $(\mathcal{G} \mathcal{K} \mathcal{L}$ function) if

i) the mapping $s \mapsto \beta(s, 0)$ is a class- $\mathcal{K}$ function;

ii) for each fixed $s \geq 0$ the mapping $t \mapsto \beta(s, t)$ is continuous, decreases to zero and there exists some $T_{\beta}(s) \in[0,+\infty)$ such that $\beta(s, t)>0$ for all $t \in\left[0, T_{\beta}(s)\right)$ and $\beta(s, t)=0$ for all $t \geq T_{\beta}(s)$.

Compared to $\mathcal{K} \mathcal{L}$ functions, a $\mathcal{G K} \mathcal{L}$ function has to be a $\mathcal{K}$ function only for $t=0$ whereas a $\mathcal{K} \mathcal{L}$ function has to be so for any fixed $t \geq 0$. Moreover, a $\mathcal{G} \mathcal{K} \mathcal{L}$ function $\beta$ not only has to be continuous and decreasing for each fixed $s$, but also has to converge to zero in a finite amount of time $T_{\beta}(s)$. Note that the definition of a $\mathcal{G K} \mathcal{L}$ function presented here differs from the one introduced in (Hong et al., 2010).

Combining the definitions of FTS/FxTS and ISS/iISS given previously, we obtain the required accelerated robust stability notions:

Definition 2.7. System (2.14) is said to be finite-time ISS (FT-ISS) if for all $x_{0} \in \mathbb{R}^{n}$ and $d \in L^{\infty}\left(\mathbb{R}_{+}, \mathbb{R}^{m}\right)$, each solution $x\left(t, x_{0}, d\right)$ is defined for $t \geq 0$ and satisfies

$$
\left\|x\left(t, x_{0}, d\right)\right\| \leq \beta\left(\left\|x_{0}\right\|, t\right)+\vartheta\left(\|d\|_{\infty}\right),
$$

where $\vartheta \in \mathcal{K}$ and $\beta \in \mathcal{G K} \mathcal{L}$ with $T_{\beta} \in C\left(\mathbb{R}_{+}, \mathbb{R}_{+}\right)$and $T_{\beta}(0)=0$. If, furthermore, $\sup _{r \in \mathbb{R}_{+}} T_{\beta}(r)<+\infty$, the system (2.14) is said to be fixed-time ISS (FxT-ISS).

Definition 2.8. System (2.14) is said to be finite-time iISS (FTiISS) if for all $x_{0} \in \mathbb{R}^{n}$ and $d \in L^{\infty}\left(\mathbb{R}_{+}, \mathbb{R}^{m}\right)$, each solution $x\left(t, x_{0}, d\right)$ is defined for $t \geq 0$ and satisfies

$$
\alpha\left(\left\|x\left(t, x_{0}, d\right)\right\|\right) \leq \beta\left(\left\|x_{0}\right\|, t\right)+\int_{0}^{t} \vartheta(\|d(s)\|) d s,
$$

where $\alpha \in \mathcal{K}_{\infty}, \vartheta \in \mathcal{K}$ and $\beta \in \mathcal{G} \mathcal{K} \mathcal{L}$ with $T_{\beta} \in C\left(\mathbb{R}_{+}, \mathbb{R}_{+}\right)$and 
$T_{\beta}(0)=0$. If, furthermore, $\sup _{r \in \mathbb{R}_{+}} T_{\beta}(r)<+\infty$, the system (2.14) is said to be fixed-time iISS (FxT-iISS).

Remark that indeed the key difference with respect to asymptotic ISS is that $\beta$ is a $\mathcal{G} \mathcal{K} \mathcal{L}$ function and that according to Definition 2.6 this implies the existence of an estimate of the settling-time function $T_{\beta}$. Note also that only the case of continuous $T_{\beta}$ is considered.

From the definition of F(x)T-(i)ISS, it follows that if the system (2.14) possesses one of these properties, then for $d=0$ it becomes $\mathrm{F}(\mathrm{x}) \mathrm{TS}$ with a continuous settling-time function.

\subsubsection{Lyapunov characterizations}

The efficiency and popularity of ISS framework is based on availability of equivalent Lyapunov characterizations.

Definition 2.9 (Sontag, 2007). A function $V \in C^{\infty}\left(\mathbb{R}^{n}, \mathbb{R}_{+}\right)$is called $I S S-L F$ for the system (2.14) if there exist $\alpha_{1}, \alpha_{2}, \alpha \in \mathcal{K}_{\infty}$ and $\chi \in \mathcal{K}$ such that

$$
\begin{gathered}
\alpha_{1}(\|x\|) \leq V(x) \leq \alpha_{2}(\|x\|), \\
D V(x) f(x, d) \leq-\alpha(\|x\|)+\chi(\|d\|)
\end{gathered}
$$

for all $x \in \mathbb{R}^{n}$ and all $d \in \mathbb{R}^{m}$. This function is called $i I S S$-LF for the system (2.14) if the above properties hold for a positive definite $\alpha \in C\left(\mathbb{R}_{+}, \mathbb{R}_{+}\right)$.

As the next lemma states, there exists an alternative definition of an ISS-LF that, depending on the family and context of the systems under study, may be more relevant by easing the analysis of stability (Dashkovskiy et al., 2011):

Lemma 2.6 (Sontag, 2007). A $V \in C^{\infty}\left(\mathbb{R}^{n}, \mathbb{R}_{+}\right)$is an ISS-LF for (2.14) if and only if there exist $\alpha_{1}, \alpha_{2}, \delta, \zeta \in \mathcal{K}_{\infty}$ such that (2.16) holds and

$$
\|x\| \geq \delta(\|d\|) \Rightarrow D V(x) f(x, d) \leq-\zeta(\|x\|)
$$

for all $x \in \mathbb{R}^{n}$ and all $d \in \mathbb{R}^{m}$. 
The following main result in the ISS theory relates the existence of an ISS/iISS-LF function with the ISS/iISS property for a given system (the equivalence has been obtained for a locally Lipschitz continuous $f$ ):

Theorem 2.7 (Sontag, 2007). The system (2.14) is (i)ISS if and only if it admits an (i)ISS-LF.

In order to check accelerated ISS/iISS properties, as usual, the above definitions need mild modifications. To this end define a relation $\eta_{1} \sim \eta_{2}$ for $\eta_{1}, \eta_{2} \in \mathcal{K}$ meaning that there are some $\epsilon>0$ and $\kappa>0$ such that $\eta_{1}(s) \geq \kappa \eta_{2}(s)$ for all $s \in[0, \epsilon)$.

Definition 2.10. A $V \in C^{1}\left(\mathbb{R}^{n}, \mathbb{R}_{+}\right)$is called a $F T$-(i)ISS-LF for the system (2.14) if there exist some $\alpha_{1}, \alpha_{2}, \alpha \in \mathcal{K}_{\infty}$ (a positive definite $\left.\alpha \in C\left(\mathbb{R}_{+}, \mathbb{R}_{+}\right)\right), \chi \in \mathcal{K}, c>0$ and $a \in[0,1)$ such that (2.16) and (2.17) hold for all $x \in \mathbb{R}^{n}$ and all $d \in \mathbb{R}^{m}$ with $\alpha(s) \sim c s^{a}$.

In the ISS case the condition (2.17) can be replaced by (2.18) according to Lemma 2.6, then $\zeta(s) \sim c s^{a}$.

In (Hong et al., 2010), some sufficient conditions for finite-time ISS with continuous settling-time function are presented. However converse results are not obtained. The following results show that if some assumptions on the Lipschitz continuity of the system outside the origin and of the settling-time function are added, then a converse result exists.

Assumption 2.1. Let on $\left(\mathbb{R}^{n} \backslash\{0\}\right) \times \mathbb{R}^{m}$ the function $f: \mathbb{R}^{n+m} \rightarrow \mathbb{R}^{n}$ be locally Lipschitz continuous and, in addition, there exists some $L \in C\left(\mathbb{R}_{+}, \mathbb{R}_{+}\right)$such that

$$
\|f(x, d)-f(x, 0)\| \leq L(\|x\|)\|d\|
$$

for all $x \in \mathbb{R}^{n}$ and all $d \in \mathbb{R}^{m}$. 
Theorem 2.8. The system (2.14) is FT-(i)ISS if it admits a FT(i)ISS-LF. Conversely, if (2.14) is FT-ISS with a Lipschitz continuous settling-time function $T$ and Assumption 2.1 is satisfied, then there exists a FT-ISS-LF function for it.

Proof. The proof for the FT-ISS case was given in (Lopez-Ramirez et al., 2020) (we need just its mild modification related with the definition of $\alpha$ or $\zeta$ ). The FT-iISS can be proven similarly.

Assume that any of the conditions of Theorem 2.8 holds and $\zeta(s)=$ $c s^{a}$, then the settling-time function $T$ satisfies

$$
T(x) \leq \frac{V^{1-a}(x)}{c_{1}(1-a)}
$$

for $d=0$ and for all $x \in \mathbb{R}^{n}$.

Example 2.7. (Lopez-Ramirez et al., 2020) Consider a system with disturbances

$$
\begin{aligned}
& \dot{x}_{1}=-\left\lfloor x_{1}\right\rceil^{\gamma}-x_{2}+d_{1} \\
& \dot{x}_{2}=x_{1}-\left\lfloor x_{2}\right\rceil^{\gamma}+d_{2}
\end{aligned}, \quad x, d \in \mathbb{R}^{2}, \gamma \in(0,1)
$$

and an ISS-LF candidate $V(x)=\frac{1}{2}\left(x_{1}^{2}+x_{2}^{2}\right)$. We have that

$$
D V(x) f(x, d) \leq-\left|x_{1}\right|^{1+\gamma}-\left|x_{2}\right|^{1+\gamma}+\|x\|\|d\|,
$$

since

$$
\left(x_{1}^{2}+x_{2}^{2}\right)^{\frac{1+\gamma}{2}} \leq\left|x_{1}\right|^{1+\gamma}+\left|x_{2}\right|^{1+\gamma}
$$

for all $x_{1}, x_{2} \in \mathbb{R}$, then

$$
D V(x) f(x, d) \leq-\|x\|^{1+\gamma}+\|x\|\|d\|
$$

and for $\|d\| \leq \frac{1}{2}\|x\|^{\gamma}$ we obtain

$$
D V(x) f(x, d) \leq-\frac{1}{2}\|x\|^{1+\gamma}=-2^{\frac{\gamma-1}{2}} V^{\frac{1+\gamma}{2}}(x) .
$$

Then, according to Theorem 2.8, the system is FT-ISS, and the settlingtime function for $d_{1}=d_{2}=0$ can be estimated as

$$
T(x) \leq \frac{2^{\frac{3-\gamma}{2}}}{1-\gamma}\left(x_{1}^{2}+x_{2}^{2}\right)^{\frac{1-\gamma}{2}} .
$$


Definition 2.11 (Lopez-Ramirez et al., 2020). A $V \in C^{1}\left(\mathbb{R}^{n}, \mathbb{R}_{+}\right)$ is called a FxT-ISS-LF for the system (2.14) if there exist some $\alpha_{1}, \alpha_{2} \in \mathcal{K}_{\infty}, \chi \in \mathcal{K}, c_{1}, c_{2}>0, a \in[0,1)$ and $b>1$ such that $(2.16)$ and

$$
\|x\| \geq \chi(\|d\|) \Rightarrow D V(x) f(x, d) \leq-c_{1} V^{a}(x)-c_{2} V^{b}(x)
$$

hold for all $x \in \mathbb{R}^{n}$ and all $d \in \mathbb{R}^{m}$.

Theorem 2.9 (Lopez-Ramirez et al., 2020). The system (2.14) is FxT-ISS if it admits a FxT-ISS-LF.

Another condition of FxT-ISS based on local homogeneity is given in (Andrieu et al., 2008).

If the Theorem 2.9 holds and $d=0$, then the settling-time function $T$ satisfies

$$
T(x) \leq \frac{1}{c_{1}(1-a)}+\frac{1}{c_{2}(b-1)} \quad \forall x \in \mathbb{R}^{n},
$$

and this bound on the settling-time is independent of $x$.

Example 2.8. The behavior of trajectories of a scalar system

$$
\dot{x}=-a x^{\alpha}-b x^{\beta}+d
$$

with $x, d \in \mathbb{R}, a, b>0, \alpha \in(0,1)$ and $\beta>1$ can be analyzed by applying a conventional Lyapunov function $V=x^{2}$, then

$$
\begin{aligned}
\dot{V} & \leq-2 a V^{\frac{\alpha+1}{2}}-2 b V^{\frac{\beta+1}{2}}+2 \sqrt{V}|d| \\
& \leq\left\{\begin{array}{ll}
-2 a V^{\frac{\alpha+1}{2}}+2 \sqrt{V}|d| & V \in[0,1] \\
-2 b V^{\frac{\beta+1}{2}}+2 \sqrt{V}|d| & V>1
\end{array},\right.
\end{aligned}
$$

and

$$
|d| \leq \frac{\min \{a, b\}}{2} \max \left\{V^{\frac{\alpha}{2}}, V^{\frac{\beta}{2}}\right\} \Rightarrow \dot{V} \leq \begin{cases}-2 a V^{\frac{\alpha+1}{2}} & V \in[0,1] \\ -2 b V^{\frac{\beta+1}{2}} & V>1\end{cases}
$$


Solving the above relation as for the example (2.11), the inequality (2.15) with

$$
\begin{gathered}
\beta(s, t)=\max \left\{0, \min \left\{\left(s^{1-\alpha}-a \frac{1-\alpha}{2} t\right)^{\frac{1}{1-\alpha}},\left(s^{1-\beta}+b \frac{\beta-1}{2} t\right)^{\frac{1}{1-\beta}}\right\}\right\}, \\
\vartheta(s)=\max \left\{\left(\frac{2 s}{\min \{a, b\}}\right)^{\frac{1}{\alpha}},\left(\frac{2 s}{\min \{a, b\}}\right)^{\frac{1}{\beta}}\right\}
\end{gathered}
$$

can be obtained. The settling-time function admits an upper estimate:

$$
\begin{aligned}
T(x) \leq T_{\beta}(s) & \leq \begin{cases}\frac{2 s^{1-\alpha}}{a(1-\alpha)} & s \in[0,1] \\
\frac{2}{a(1-\alpha)}+\frac{2\left(1-s^{1-\beta}\right)}{b(\beta-1)} & s>1\end{cases} \\
\leq & \frac{2}{a(1-\alpha)}+\frac{2}{b(\beta-1)}
\end{aligned}
$$

as in (2.20), and the system has a fixed-time convergence rate. Note that $\beta(s, 0)=s$ is a class- $\mathcal{K}_{\infty}$ function, $\beta(s, t)=0$ for all $t \geq T_{\beta}(s)$, and $\beta(s, t)$ is decreasing for each fixed $s \in \mathbb{R}_{+}$, hence, $\beta$ is a class- $\mathcal{G K} \mathcal{L}$ function and the system (2.21) is FxT-ISS.

\subsection{Discussions}

Summarizing and complementing the existing results, this section presents the definitions of different accelerated stability notions together with their Lyapunov characterizations. Existence of respective Lyapunov functions is necessary and sufficient if the characterization is explicitly dependent on $\dot{V}$ or the settling-time function is continuous. For (nearly) fixed-time stability, the corresponding necessary and sufficient Lyapunov conditions are derived under additional assumption of existence and uniqueness of solutions in backward time out the origin. A sufficient condition for finite/fixed-time stabilization of affine systems, analogous to previous results on asymptotic stabilization via CLF, will be also discussed in Section 4.1. The robust extensions (in ISS sense) of stronger-than-asymptotic stability concepts are described at the end.

There exist some related development, for instance, to time-varying systems (Moulay and Perruquetti, 2008), which are omitted here for brevity. There are also relaxed accelerated converge rates called hyperexponential, which means an asymptotic decay with a velocity faster 
than any exponential, but they have more importance for discrete-time systems (Sanchez et al., 2019; Sanchez et al., 2020) or time-delay models (Polyakov et al., 2015b).

Despite rather intensive investigations in the domain during the last decade, a lot of open problems are still left. For example, a necessary condition for (nearly) fixed-time stability and stabilization with continuous settling-time function, without assuming completeness of these properties, is missed. The necessary Lyapunov characterizations for FxT-ISS and Lyapunov conditions for FxT-iISS do not exist yet. 


\section{Homogeneity}

The approach for analysis and design of faster-than-asymptotically convergent systems based on the use of Lyapunov functions, which is presented in the previous section, is generic and very powerful. However, it has a strong drawback related with the choice of a Lyapunov function in each particular application. Such a shortage is common for the Lyapunov function method: for linear systems and for some classes of nonlinear ones there are well-established canonical forms of Lyapunov functions (whose existence is sufficient and/or necessary for stability of the given groups of dynamical models), but it is very difficult to find a Lyapunov function for a general nonlinear system. As we have seen from the examples given above, the systems having finite/fixed-time convergence rates are strongly nonlinear and, frequently, to find a Lyapunov function for them is an issue. That is why any alternative approach for evaluation of accelerated convergence is important, and a very popular in application method is based on the theory of homogeneous systems introduced in this chapter.

After definition of homogeneity, we will present the important and useful properties of homogeneous dynamical systems. The advantages of stability and robustness analysis by applying homogeneity will be 
highlighted. Next, the concept of homogeneous approximations will be given (it enlarges the class of systems whose behavior can be investigated by applying the theory of homogeneity) together with some generalizations.

\subsection{Definitions and various concepts}

In this section we will present several definitions of homogeneity, starting from the simplest one (which is also the most restrictive), untill more sophisticated concepts (which include more general classes of dynamics). The class of homogeneous norms (a kind of distance functions possessing the homogeneity properties) will be introduced.

\subsubsection{The classical concept of homogeneity}

Symmetry is a type of invariance when some characteristics of an object do not change under a certain set of transformations. It occurs in many branches of mathematics. The simplest example of a symmetry can be found in the geometry as an invariance of geometric figures with respect to rotations, translations or dilations. It is well known that both the size and the shape of the figure are invariant with respect to rotations and translations, while the dilation does not change the shape.

The symmetry of shapes with respect to dilations can be discovered for level sets of the so-called homogeneous functions. The symmetry of a function $f$ with respect to the (uniform) dilation of its argument

$$
x \rightarrow \lambda x,
$$

where $\lambda>0$ is the scaling factor, is known as (standard) homogeneity:

$$
f(\lambda x)=\lambda f(x), \quad \forall \lambda>0, \quad \forall x .
$$

In other words, homogeneity is a dilation symmetry. All linear functions are homogeneous with respect to the uniform dilation. In this chapter it is shown that homogeneous nonlinear mappings are rather similar to linear ones. However, they have their own specific features which could be useful for an advanced control system design. 
In 18th century, a homogeneity with respect to the uniform dilation was studied by Leonhard Euler. His notion of homogeneity is well known today in the context of the so-called homogeneous polynomials.

Definition 3.1 (L. Euler, 18th century). A function $f: \mathbb{R}^{n} \rightarrow \mathbb{R}^{n}$ is said to be standard homogeneous if there exists a number $\nu \in \mathbb{R}$ such that

$$
f(\lambda x)=\lambda^{\nu} f(x), \quad \forall \lambda>0, \quad \forall x \in \mathbb{R}^{n} .
$$

The number $\nu$ is called the homogeneity degree of the function $f$.

According to this definition, any linear function has the homogeneity degree 1 , but the quadratic one

$$
x=\left(x_{1}, x_{2}\right)^{\top} \stackrel{f}{\rightarrow} x_{1}^{2}+x_{1} x_{2}+x_{2}^{2}
$$

is homogeneous of the degree 2. The level sets of $f$ are ellipsoids centered at the origin. They are symmetric with respect to the uniform dilation.

Euler's Homogeneous Function Theorem given below is one of famous results underlying the modern theory of homogeneous systems.

Theorem 3.1 (L. Euler, 18th century). A continuously differentiable function $f: \mathbb{R}^{n} \rightarrow \mathbb{R}$ is standard homogeneous of a degree $k$ if and only if $\frac{\partial f}{\partial x} x=k f(x), x \in \mathbb{R}^{n}$.

From this theorem, in particular, we conclude that the homogeneity of a function is inherited by its derivatives. In fact, the dilation symmetry can be inherited by other mathematical objects induced by homogeneous functions. For example, solutions of homogeneous differential equations and inclusions are also symmetric (homogeneous) in a certain sense. 


\section{Standard homogeneous differential equations}

Let us consider the simplest scalar ordinary differential equation (ODE) with the standard homogeneous right-hand side

$$
\dot{x}=-x^{\nu}, \quad t>0, \quad \nu=p / q,
$$

where $p$ is an odd integer and $q$ is an even natural number. Its solution with the initial condition $x(0)=x_{0} \in \mathbb{R}$ is given by

$$
x\left(t, x_{0}\right)=\frac{x_{0}}{\left(1+(\nu-1) t\left|x_{0}\right|^{\nu-1}\right)^{1 /(\nu-1)}} .
$$

Hence, we easily derive the symmetry of solutions with respect to the simultaneous dilation of the initial condition and the time variable $t$ :

$$
x\left(\lambda^{1-\nu} t, \lambda x_{0}\right)=\lambda x\left(t, x_{0}\right), \quad \lambda>0 .
$$

Notice that the time scaling factor depends on the homogeneity degree $\nu$. In fact, it is easy to check that the mentioned symmetry of solutions can be established for any standard homogeneous differential equation. This result is proven for a more general class of systems, which are homogeneous in a generalized sense (see below).

Theorem 3.2. Let $f: \mathbb{R}^{n} \rightarrow \mathbb{R}^{n}$ be a continuous standard homogeneous vector field of a degree $\nu \in \mathbb{R}$ such that the Cauchy problem

$$
\dot{x}=f(x), \quad x(0)=x_{0} \in \mathbb{R}^{n}
$$

admits a solution $x\left(t, x_{0}\right)$ defined for all $t>0$. Then

$$
x\left(\lambda^{1-\nu} t, \lambda x_{0}\right)=\lambda x\left(t, x_{0}\right), \quad \lambda>0,
$$

where $x\left(\cdot, \lambda x_{0}\right)$ is a solution to the same problem with the scaled initial condition $x(0)=\lambda x_{0}$.

Homogeneity simplifies an analysis and design of nonlinear control systems, since the homogeneous vector fields have many properties similar to linear ones. For example, $x=0$ is always an equilibrium of 


\begin{tabular}{|c|c|c|}
\hline & $\begin{array}{c}\text { Linear System } \\
\dot{x}=A x \\
A \in \mathbb{R}^{n \times n}\end{array}$ & $\begin{array}{c}\text { Homogeneous System } \\
\dot{x}=f(x) \\
f(\lambda x)=\lambda^{\nu} f(x)\end{array}$ \\
\hline Trajectory Scaling & $x\left(t, \lambda x_{0}\right)=\lambda x\left(t, x_{0}\right)$ & $x\left(t, \lambda x_{0}\right)=\lambda x\left(\lambda^{\nu-1} t, x_{0}\right)$ \\
\hline $\begin{array}{c}\text { Stability } \\
\text { Local } \Leftrightarrow \text { Global }\end{array}$ & $\checkmark$ & $\checkmark$ \\
\hline Lyapunov Function & $\begin{array}{c}\text { Quadratic } \\
\text { Stability } \Rightarrow \text { Robustness } \\
\text { Input-to-State Stability) }\end{array}$ & $\dot{x}=A x+D w$ \\
\hline Convergence Rate & $w \in L^{\infty}$ & $\begin{array}{c}\text { Homogeneous } \\
=x^{\top} \Xi^{\top}(x) P \Xi(x) x, P \succ 0 \\
\Xi(\lambda x)=\Xi(x) \in \mathbb{R}^{n \times n}\end{array}$ \\
\hline $\begin{array}{c}\dot{x}=f(x, w), \\
\tilde{f}=\left(\begin{array}{l}f \\
0\end{array}\right)-\text { homogeneous }\end{array}$ \\
\hline $\begin{array}{c}\text { Consistent Discretization } \\
\text { preserves convergence rate }\end{array}$ & Exponential & $\begin{array}{c}\text { Finite-time }(\nu<1) \\
\text { Exponential }(\nu=1) \\
\text { Nearly fixed-time }(\nu>1)\end{array}$ \\
\hline
\end{tabular}

Table 3.1: Some properties of linear and homogeneous systems

a continuous homogeneous system. If there exists another equilibrium $x^{*} \neq 0$ then the set $M=\left\{x \in \mathbb{R}^{n}: x=\lambda x^{*}, \lambda>0\right\}$ is, at least, weakly (for a part of solutions) invariant and $f(x)=0$ for all $x \in M$. In the case of linear systems, the set $M$ is a linear subspace.

From the latter theorem we immediately conclude that any local property (e.g., a local stability and the existence of solutions for small initial data) can always be expanded globally. Similarly to linear systems, the robustness (input-to-state stability) of a homogeneous system can be granted by its stability in the disturbance-free case (see Subsection 3.4 for more details). Table 3.1 compares some properties of linear and nonlinear homogeneous control systems.

Homogeneity degree allows some additional qualitative analysis of ODEs to be done easily. For instance, finite-time or nearly fixed-time stability can be derived from the homogeneity degree. Indeed, if $\nu=1 / 3$ then the solution of the scalar homogeneous system $\dot{x}=-x^{\nu}$ is given by

$$
x\left(t, x_{0}\right)=\left\{\begin{array}{cll}
\left(\left|x_{0}\right|^{\frac{2}{3}}-\frac{2}{3} t\right)^{\frac{3}{2}} & \text { if } & t \in\left[0, \frac{3}{2}\left|y_{0}\right|^{\frac{2}{3}}\right), \\
0 & \text { if } & t \geq \frac{3}{2}\left|x_{0}\right|^{\frac{2}{3}}
\end{array}\right.
$$


Obviously, it converges to zero in a finite time $T\left(x_{0}\right)=\frac{3}{2}\left|x_{0}\right|^{2 / 3}$. However, if $\nu>1$ then each trajectory of the system converges into any neighborhood of the origin in a fixed time independent of the initial condition, namely,

$$
\left|x\left(t, x_{0}\right)\right|<r, \quad \forall t>\frac{1}{r^{\nu-1}(\nu-1)}
$$

for any $x_{0} \in \mathbb{R}$ and any $r>0$. In fact, these properties can be established in a more general case. Namely, if the origin of a homogeneous system is asymptotically stable then each trajectory of the system

a) vanishes in a finite time provided that $\nu<1$;

b) converges to zero exponentially if $\nu=1$;

c) converges to a neighborhood of the origin in a fixed time independent of the initial condition if $\nu>1$.

\subsubsection{Weighted homogeneity}

The standard homogeneity has been introduced by means of the uniform dilation $x \rightarrow \lambda x, \lambda>0$. It is clear that if we change the dilation rule then another type of homogeneity can be defined. The weighted dilation (studied in Zubov, 1958) of the vector $x=\left(x_{1}, x_{2}, \ldots, x_{n}\right)^{\top} \in \mathbb{R}^{n}$ is the simplest case of the so-called generalized (non-uniform) dilation:

$$
\left(x_{1}, x_{2}, \ldots, x_{n}\right) \rightarrow\left(\lambda^{r_{1}} x_{1}, \lambda^{r_{2}} x_{2}, \ldots, \lambda^{r_{n}} x_{n}\right),
$$

where $\lambda>0$, as before, is the scaling factor and the positive numbers $r_{1}, r_{2}, \ldots, r_{n}$ are the weights, which specify dilation rates along different coordinates. If $r_{1}=r_{2}=\ldots=r_{n}=1$ then the weighted dilation becomes uniform. The introduced transformation of coordinates

$$
x \rightarrow \Lambda x
$$

is a linear mapping $\mathbb{R}^{n} \rightarrow \mathbb{R}^{n}$ defined by the dilation matrix

$$
\Lambda=\left(\begin{array}{cccc}
\lambda^{r_{1}} & 0 & \ldots & 0 \\
0 & \lambda^{r_{2}} & \ldots & 0 \\
\ldots & \ldots & \ldots & \ldots \\
0 & 0 & \ldots & \lambda^{r_{n}}
\end{array}\right)
$$


The symmetry (homogeneity) of a scalar-valued function with respect to the weighted dilation can be identified analogously to the uniform case.

Definition 3.2 (Zubov, 1958). A function $f: \mathbb{R}^{n} \rightarrow \mathbb{R}$ is homogeneous with respect to the weighted dilation $\Lambda$ if

$$
f(\Lambda x)=\lambda^{\nu} f(x), \quad x \in \mathbb{R}^{n}, \quad \lambda>0,
$$

where $\nu \in \mathbb{R}$ is the homogeneity degree.

The weighted dilation extends the class of homogeneous functions under consideration. For example, the polynomial function

$$
\left(x_{1}, x_{2}\right) \stackrel{f}{\rightarrow} x_{1}^{2}+x_{1} x_{2}^{2}+x_{2}^{4}
$$

is homogeneous with respect to the weighted dilation

$$
\left(x_{1}, x_{2}\right) \rightarrow\left(\lambda^{2} x_{1}, \lambda x_{2}\right),
$$

but it is not homogeneous with respect to the uniform one

$$
\left(x_{1}, x_{2}\right) \rightarrow\left(\lambda x_{1}, \lambda x_{2}\right) .
$$

Definition 3.3 (Zubov, 1958). A vector field $f: \mathbb{R}^{n} \rightarrow \mathbb{R}^{n}$ is said to be weighted homogeneous if

$$
f(\Lambda x)=\lambda^{\mu} \Lambda f(x), \quad x \in \mathbb{R}^{n}, \quad \lambda>0,
$$

where $\mu \in \mathbb{R}$ is a homogeneity degree of the vector field $f$.

Notice that, due to the non-uniformity of the weighted dilation, the definition of the weighted homogeneous vector field $\mathbb{R}^{n} \rightarrow \mathbb{R}^{n}$ differs from Definition 3.1. However, it can be shown (see remarks after Theorem 3.10) that any weighted (in fact, even a more generalized) 
homogeneous system is topologically equivalent (homeomorphic) to a standard homogeneous one. Consequently, it demonstrates the same properties like the symmetry of solutions, the equivalence of local and global properties, the finite-time convergence for $\mu<0$, the practical fixed-time convergence for $\mu>0$, etc.

Weighted homogeneous models frequently appear in control theory and applications. For example, the classical minimum time control problem

$$
T \rightarrow \min _{u}
$$

subject to

$$
\begin{cases}\dot{x}_{1}=x_{2}, & u \in L^{\infty}((0, T), \mathbb{R}) \text { such that }|u(t)| \leq 1, \\ \dot{x}_{2}=u, & x_{1}(T)=x_{2}(T)=0\end{cases}
$$

has the solution (see, e.g. Chernous'ko et al., 2008) in the form of the weighted homogeneous feedback

$$
u=-\operatorname{sign}\left(\left|x_{2}\right| x_{2}+2 x_{1}\right) .
$$

Indeed, $u\left(\lambda^{2} x_{1}, \lambda x_{2}\right)=u\left(x_{1}, x_{2}\right)$, i.e. $u$ is the weighted homogeneous function with the zero degree.

The weighted dilations and the weighted homogeneity in $\mathbb{R}^{n}$ allow some important results to be obtained about

- the global expansion of a local stability and the existence of homogeneous Lyapunov functions Zubov, 1958, Rosier, 1992;

- controllability, stabilizability and observability of nonlinear systems Kawski, 1990, Sepulchre and Aeyels, 1996a, Sepulchre and Aeyels, 1996b, Grune, 2000;

- controllers and observers design Andreini et al., 1988, Coron and Praly, 1991, Bhat and Bernstein, 2005, Perruquetti et al., 2008, Andrieu et al., 2008, Polyakov et al., 2015a, Lopez-Ramirez et al., 2018, Polyakov, 2018;

- robustness analysis of both delay-free Ryan, 1995, Andrieu et al., 2008, Bernuau et al., 2013 and time-delay systems Aleksandrov et al., 2012, Zimenko et al., 2017. 
- the high-order sliding mode algorithms Levant, 2005, Orlov, 2005, Bernuau et al., 2014b.

\subsubsection{Geometric homogeneity}

Let, for simplicity, the scaling factor $\lambda>0$ be denoted as follows $\lambda=e^{s}$ with $s \in \mathbb{R}$, where $e=2.71828 \ldots$ is the Euler number. In this case, any standard homogeneous $f: \mathbb{R}^{n} \rightarrow \mathbb{R}$ is invariant with respect to the scaling of its argument

$$
f\left(e^{s} x\right)=e^{\nu s} f(x), \quad s \in \mathbb{R}, \quad x \in \mathbb{R}^{n},
$$

where $\nu \in \mathbb{R}$ is, as before, the homogeneity degree. From the previous section we have understood that instead of the uniform scaling of the argument

$$
x \rightarrow e^{s} x
$$

we may consider a non-uniform one

$$
x \rightarrow \mathbf{d}(s) x
$$

where an operator $\mathbf{d}(s)$ maps $\mathbb{R}^{n}$ onto $\mathbb{R}^{n}$ and $s \in \mathbb{R}$ is a parameter of the dilation. In this case, the weighted dilation $x \rightarrow \Lambda x$ becomes $x \rightarrow \mathbf{d}(s) x, s \in \mathbb{R}$, where

$$
\mathbf{d}(s)=\left(\begin{array}{cccc}
e^{r_{1} s} & 0 & \cdots & 0 \\
0 & e^{r_{2}} & \cdots & 0 \\
\cdots & \cdots & \cdots & 0 \\
0 & 0 & \cdots & e^{r_{n}}
\end{array}\right), r_{i}>0 .
$$

In the general case, to be a dilation, the family of operators $\mathbf{d}(s)$ must satisfy some conventional restrictions (see Husch, 1970).

Definition 3.4 (Kawski, 1991). A family of operators $\mathbf{d}(s): \mathbb{R}^{n} \rightarrow \mathbb{R}^{n}$, $s \in \mathbb{R}$ is said to be a dilation group (or, simply, dilation) in $\mathbb{R}^{n}$ if

- $\mathbf{d}(0) x=x, \mathbf{d}(s+t) x=\mathbf{d}(s) \mathbf{d}(t) x, \forall t, s \in \mathbb{R}, \forall x \in \mathbb{R}^{n} ;$

- $\|\mathbf{d}(s) x\| \rightarrow 0$ as $s \rightarrow-\infty$;

- $\|\mathbf{d}(s) x\| \rightarrow+\infty$ as $s \rightarrow+\infty$ for $x \neq \mathbf{0}$. 
In Khomenuk, 1961, Kawski, 1991, Rosier, 1993, the dilation d is suggested to be generated as a flow of a $C^{1}$ vector field $g: \mathbb{R}^{n} \rightarrow \mathbb{R}^{n}$. Namely, let us consider

$$
\dot{\phi}(s)=g(\phi(s)), \quad \phi(0)=x, \quad s \in \mathbb{R} .
$$

and assume that the origin of $\dot{z}=-g(z)$ is globally uniformly asymptotically stable. The latter means that the origin of (3.1) is unstable and any solution $\phi_{x}(s)$ with the initial condition $\phi(0)=x \neq \mathbf{0}$ is unique and can be treated as a dilation of $x$, i.e. $\mathbf{d}(s): \mathbb{R}^{n} \rightarrow \mathbb{R}^{n}$ is defined as

$$
\mathbf{d}(s) x:=\phi_{x}(s), \quad x \in \mathbb{R}^{n} .
$$

Indeed, $\mathbf{d}(0) x=x$ and $\|\mathbf{d}(s) x\| \rightarrow+\infty$ as $s \rightarrow+\infty$ for $x \neq 0$ due to instability of (3.1) in the forward time. Moreover, $\|\mathbf{d}(s) x\| \rightarrow 0$ as $s \rightarrow-\infty$ due to global asymptotic stability of (3.1) in the backward time.

Definition 3.5 (Kawski, 1991, Rosier, 1993). A dilation $\mathbf{d}(s)$ is said to be geometric if it is generated by an Euler vector field (a $C^{1}$ vector field $g: \mathbb{R}^{n} \rightarrow \mathbb{R}^{n}$ is said to be Euler vector field if it is complete and $\dot{\phi}=-g(\phi)$ is globally asymptotically stable).

\section{Linear dilations}

In this chapter we deal also with the so-called linear geometric dilations (or, simply, linear dilation), which require the vector fields $g$ to be linear:

$$
\dot{\phi}(s)=G_{\mathbf{d}} \phi(s),
$$

where $G_{\mathbf{d}} \in \mathbb{R}^{n \times n}$ is an anti-Hurwitz matrix known as the generator of the linear dilation group.

In this case, the dilation $\mathbf{d}$ is given by the matrix exponential

$$
\mathbf{d}(s):=e^{G_{\mathbf{d}} s}=\sum_{i=0}^{\infty} \frac{s^{i} G_{\mathbf{d}}^{i}}{i !} .
$$


The dilation (3.2) is studied in the literature in the context of the so-called homogeneous Lie groups (see Fischer and Ruzhanky, 2016 and references therein).

Definitions of $\mathbf{d}$-homogeneous functions and vector fields are given similarly to the case of the weighted homogeneity.

Definition 3.6 (Kawski, 1991). A function $h: \mathbb{R}^{n} \rightarrow \mathbb{R}$ is said to be d-homogeneous of a degree $\nu \in \mathbb{R}$ if

$$
h(\mathbf{d}(s) u)=e^{\nu s} h(u) \quad \text { for } \quad s \in \mathbb{R}, \quad u \in \mathcal{D}(h),
$$

where $\mathbf{d}$ is a geometric dilation in $\mathbb{R}^{n}$.

The homogeneous norm studied in the previous section is an example of the so-called homogeneous functional.

Definition 3.7 (Kawski, 1991). A vector field $f: \mathbb{R}^{n} \rightarrow \mathbb{R}^{n}$ is said to be $\mathbf{d}$-homogeneous of a degree $\nu \in \mathbb{R}$ if

$$
f(\mathbf{d}(s) x)=e^{\nu s} \mathbf{d}(s) f(x) \quad \text { for } \quad s \in \mathbb{R}, \quad x \in \mathbb{R}^{n},
$$

where $\mathbf{d}$ is a geometric dilation in $\mathbb{R}^{n}$.

\section{Monotone dilations}

The dilation is an a operation, which has a clear geometric interpretation. For analysis and design of homogeneous control systems it is useful to know how the dilation interlaces with the topology of the space (e.g. with a norm). The monotonicity of linear dilations has been introduced originally for Banach spaces (see Polyakov et al., 2018).

Definition 3.8. A geometric dilation $\mathbf{d}$ in $\mathbb{R}^{n}$ is said to be 
- monotone if the function $s \rightarrow\|\mathbf{d}(s) x\|$ is monotone, $\forall x \in$ $\mathbb{R}^{n}$

- strictly monotone if the function $s \rightarrow \sup _{\|x\|=1}\|\mathbf{d}(s) x\|$ is strictly monotone.

Notice that in the case of linear dilations the strict monotonicity equivalently defined as follows: there exists $\beta>0$ such that $\sup _{\|x\|=1}\|\mathbf{d}(s) x\| \leq$ $e^{\beta s}, \forall s \leq 0$.

Notice that the monotonicity of a dilation implies the uniqueness of the homogeneous projection of a point to the unit sphere (see Proposition 3.3) and depends on the norm in $\mathbb{R}^{n}$.

Corollary 3.3 (Polyakov, 2018). Let $\mathbf{d}$ be a linear dilation in the Euclidean space $\mathbb{R}^{n}$ with the norm $\|x\|_{P}=\sqrt{x^{\top} P x}, x \in \mathbb{R}^{n}$, where $0 \prec P=P^{\top} \in \mathbb{R}^{n \times n}$ is a positive definite symmetric matrix. The dilation $\mathbf{d}$ is strictly monotone if and only if the following linear matrix inequality holds

$$
P G_{\mathbf{d}}+G_{\mathbf{d}}^{\top} P \succ 0,
$$

where $G_{\mathbf{d}} \in \mathbb{R}^{n}$ is the generator of the dilation $\mathbf{d}$. Moreover, one has

$$
\begin{aligned}
& e^{\alpha s} \leq\lfloor\mathbf{d}(s)\rfloor \leq\|\mathbf{d}(s)\| \leq e^{\beta s} \quad \text { if } \quad s \leq 0, \\
& e^{\beta s} \leq\lfloor\mathbf{d}(s)\rfloor \leq\|\mathbf{d}(s)\| \leq e^{\alpha s} \quad \text { if } \quad s \geq 0
\end{aligned}
$$

where

$$
\alpha=\frac{1}{2} \lambda_{\max }\left(P^{\frac{1}{2}} G_{\mathbf{d}} P^{-\frac{1}{2}}+P^{-\frac{1}{2}} G_{\mathbf{d}}^{\top} P^{\frac{1}{2}}\right)>0
$$

and

$$
\beta=\frac{1}{2} \lambda_{\min }\left(P^{\frac{1}{2}} G_{\mathbf{d}} P^{-\frac{1}{2}}+P^{-\frac{1}{2}} G_{\mathbf{d}}^{\top} P^{\frac{1}{2}}\right)>0 .
$$

Notice that any linear dilation in $\mathbb{R}^{n}$ is strictly monotone in $\mathbb{R}^{n}$ provided that a norm in $\mathbb{R}^{n}$ is selected according to the latter corollary. For shortness, below we omit the word "strictly" when we talk about monotone linear dilations $\mathbb{R}^{n}$. 


\subsubsection{Homogeneous norms}

Further, we denote by $\mathbb{H}$ a real Hilbert space.

Definition 3.9. A continuous function $p: \mathbb{H} \rightarrow[0,+\infty)$ is said to be a $\mathbf{d}$-homogeneous norm in $\mathbb{R}^{n}$ if

- $p(x) \rightarrow 0$ as $x \rightarrow \mathbf{0}$;

- $p( \pm \mathbf{d}(s) x)=e^{s} p(x)>0$ for $x \in \mathbb{H} \backslash\{\mathbf{0}\}, s \in \mathbb{R}$;

where $\mathbf{d}$ is a dilation group.

Obviously, the homogeneous norm is not even a semi-norm in the classical sense, since the triangle inequality does nor hold in the general case. However, many authors (Kawski, 1990, Grune, 2000, Nakamura et al., 2002, Efimov and Perruquetti, 2011, Polyakov et al., 2018) call the functions satisfying the latter definition by homogeneous norms. We follow this tradition accepted in the control theory.

For the weighted dilation $\mathbf{d}(s)=\operatorname{diag}\left\{e^{r_{1} s}, e^{r_{2} s}, \ldots, e^{r_{n} s}\right\}$ a homogeneous norm $p: \mathbb{R}^{n} \rightarrow[0,+\infty)$ can be defined as follows (see Kawski, 1990)

$$
p(u)=\sum_{i=1}^{n}\left|u_{i}\right|^{\frac{1}{r_{i}}}, \quad u=\left(u_{1}, u_{2}, \ldots, u_{n}\right)^{\top} \in \mathbb{R}^{n} .
$$

Obviously, the given function $p$ is continuous and $p(0)=0$. Moreover, $p(\mathbf{d}(s) u)=e^{s} p(u)$ for any $u \in \mathbb{R}^{n}$.

Definition 3.10 (Polyakov, 2018). The continuous function $\|\cdot\|_{\mathbf{d}}$ : $\mathbb{R}^{n} \rightarrow[0,+\infty)$ defined as

$$
\|u\|_{\mathbf{d}}=e^{s_{u}}, \text { where } s_{u} \in \mathbb{R}:\left\|\mathbf{d}\left(-s_{u}\right) u\right\|=1,
$$

is called the canonical homogeneous norm in $\mathbb{R}^{n}$, where $\mathbf{d}$ is a monotone dilation on $\mathbb{R}^{n}$ and $\|\cdot\|$ is a (canonical) norm in $\mathbb{R}^{n}$. 
Obviously, the canonical homogeneous norm is well-defined provided that the dilation $\mathbf{d}$ is monotone. For linear monotone dilations it is shown that $\|\cdot\|_{\mathbf{d}}$ is continuous on $\mathbb{R}^{n}$ and continuously differentiable on $\mathbb{R}^{n} \backslash\{\mathbf{0}\}$ provided the original norm $\|\cdot\|$ is differentiable on $\mathbb{R}^{n} \backslash\{\mathbf{0}\}$ (see Lemma 3.19). Moreover, it defines a norm in a linear vector space $\tilde{\mathbb{R}}^{n}$ homeomorphic to $\mathbb{R}^{n}$ (see Theorem 3.20 for more details).

\subsection{Properties of homogeneous systems}

Let $\nu$ be an Euler vector field and $\mathbf{d}$ be a geometric dilation (probably nonlinear) generated by $\nu$. In this case the classical Euler homogeneous function theorem takes the following form.

Theorem 3.4 (Kawski, 1991). A $C^{1}$ function $h: \mathbb{R}^{n} \rightarrow \mathbb{R}$ is dhomogeneous of a degree $\nu$ if and only if

$$
\mathcal{L}_{g} h=\nu h,
$$

where $\mathcal{L}_{g} h:=\frac{\partial h}{\partial x} g$ denotes the Lie derivative of the function $h$ along the vector field $g$.

A $C^{1}$ vector field $f: \mathbb{R}^{n} \rightarrow \mathbb{R}^{n}$ is $\mathbf{d}$-homogeneous of a degree $\nu$ if and only if

$$
[g, f]=\nu f,
$$

where $[g, f]:=\frac{\partial f}{\partial x} g-\frac{\partial g}{\partial x} f$ denotes Lie brackets of two vector fields.

If $\mathbf{d}$ is a linear geometric dilation then $g(x)=G_{\mathbf{d}} x$ and the above identity becomes $\frac{\partial f}{\partial x} G_{\mathbf{d}} z=\left(\nu I+G_{\mathbf{d}}\right) f(x)$, which generalizes the Euler's homogenenous function theorem.

One of main features of homogeneous functions and vector field is that any local property of a homogeneous vector field in $\mathbb{R}^{n}$ can be expanded globally. 
Proposition 3.1 (Polyakov, 2018). Let $\mathbf{d}$ be a linear geometric dilation. A d-homogeneous vector field $f: \mathbb{R}^{n} \rightarrow \mathbb{R}^{n}$ is continuous (locally Lipschitz continuous or differentiable) on $\mathbb{R}^{n} \backslash\{\mathbf{0}\}$ if and only if it is continuous (Lipschitz continuous or differentiable) on $S$.

The properties of homogeneous functions at zero can be established using the homogeneity degree and the so-called spectral abscissa (at $x=0$ ) of the vector field $g$ generating the geometric dilation $\mathbf{d}$.

Theorem 3.5 (Bhat and Bernstein, 2005). Let $\mathbf{d}$ be a geometric dilation generated by an Euler vector field $g: \mathbb{R}^{n} \rightarrow \mathbb{R}^{n}$.

Let $h: \mathbb{R}^{n} \rightarrow \mathbb{R}$ be a $\mathbf{d}$-homogeneous function of a degree $\nu \in \mathbb{R}$ and $h \in C\left(\mathbb{R}^{n} \backslash\{\mathbf{0}\}, \mathbb{R}\right)$.

- If $\nu<0$, then $h$ is continuous on $\mathbb{R}^{n}$ if and only if $h \equiv 0$.

- If $\nu=0$, then $h$ is continuous on $\mathbb{R}^{n}$ if and only if $h \equiv h(0)$.

- If $\nu>0$, then $h$ is continuous on $\mathbb{R}^{n}$.

- If $\nu>0$, then $h$ is Hölder continuous at zero.

- If $\nu>\bar{\sigma}$ then $h$ is Frechét differentiable at $x=0$ with the zero Frechét derivative, where $\bar{\sigma}$ is the maximal of the real parts of the eigenvalues of the matrix $\left.\frac{\partial g(x)}{\partial x}\right|_{x=0}$.

Euler function theorem, in particular, implies that derivatives of smooth $\mathbf{d}$-homogeneous vector fields inherit the $\mathbf{d}$-homogeneity. The same hold for flows of $\mathbf{d}$-homogeneous vector fields (i.e., for solutions of $\mathbf{d}$-homogeneous systems). 
Theorem 3.6 (Kawski, 1991). Let $x\left(\cdot, x_{0}\right):[0, T) \rightarrow \mathbb{R}^{n}$ be a solution of ODE

$$
\dot{x}=f(x),
$$

with the initial condition $x(0)=x_{0} \in \mathbb{R}^{n}$, where $f: \in \mathbb{R}^{n \times n}$ is a d-homogeneous vector field of a degree $\nu \in \mathbb{R}$ and $T \leq+\infty$. Then for any $s \in \mathbb{R}$ one has

$$
x\left(t, \mathbf{d}(s) x_{0}\right)=\mathbf{d}(s) x\left(e^{\nu s} t, x_{0}\right), \quad \forall t \in\left[0, e^{-\nu s} T\right),
$$

where $x\left(\cdot, \mathbf{d}(s) x_{0}\right)$ denotes a solution of the same ODE with the initial condition $x(0)=\mathbf{d}(s) x_{0}$.

Moreover, if the ODE has a unique solution for $x(0)=x_{0}$ then it has a unique solution for $x(0)=\mathbf{d}(s) x_{0}$ for any $s \in \mathbb{R}$.

The latter theorem immediately implies that any local property ODE can be expanded globally, e.g., local (on the initial data) existence/uniqueness of solutions guarantees the global existence of solutions; local stability always implies the global one, etc.

\subsection{Stability Analysis}

First, let us notice that the homogeneity degree specifies a convergence rate of asymptotically stable homogeneous system (see Zubov, 1964).

Theorem 3.7. Let $\mathbf{d}$ be a geometric dilation in $\mathbb{R}^{n}$ and let $f$ be a d-homogeneous vector field of a degree $\nu$. If the origin of the system (3.8) is asymptotically stable then

- for $\nu<0$ it is globally uniformly finite-time stable;

- for $\nu=0$ it is globally uniformly exponentially stable provided that $\mathbf{d}$ is a linear geometric dilation ;

- for $\nu>0$ it is globally uniformly nearly fixed-time stable. 
Proof. This result was proven originally in Nakamura et al., 2002 for the weighted dilation. Theorem 3.26 extends it to linear geometric dilations. The case of an arbitrary geometric dilation with $\nu<0$ was studied in Bhat and Bernstein, 2005 using Theorem 3.9. The case $\nu>0$ can be treated similarly.

Notice that the restriction on the geometric dilation to be linear is important in the case $\nu=0$, since in the general case $\mathbf{d}$-homogeneity with the zero degree does not imply exponential convergence of a stable homogeneous system. Indeed, the scalar system $\dot{x}=-x^{3} /\left(1+x^{2}\right)$ is globally uniformly asymptotically (but not exponentially) stable and d-homogeneous of the zero degree with respect to a dilation $\mathbf{d}$ generated by the Euler vector field $g(x)=x^{3} /\left(1+x^{2}\right)$ (see Theorem 3.4).

Similar to linear systems, an invariance property implies stability for homogeneous ODEs.

Theorem 3.8 (Bhat and Bernstein, 2005). Let $f$ be a d-homogeneous continuous vector field. The origin of the system (3.8) is globally uniformly asymptotically stable if and only if this system has a strictly positively invariant compact set $\Omega \subset \mathbb{R}^{n}$ such that $\mathbf{0} \in \Omega$.

Since solutions of homogeneous ODEs inherit the dilation symmetry, it is expectable that Lyapunov functions of stable homogeneous ODEs can be homogeneous as well.

Theorem 3.9 (Rosier, 1993, Bhat and Bernstein, 2005). Let $\mathbf{d}$ be a geometric dilation in $\mathbb{R}^{n}$. Let the vector field $f: \mathbb{R}^{n} \rightarrow \mathbb{R}^{n}$ be continuous and $\mathbf{d}$-homogeneous of a degree $\nu \in \mathbb{R}$. Then the following two claims are equivalent:

- the origin of the system (3.8) is asymptotically stable; 
- there exists a d-homogeneous Lyapunov function $V \in$ $C^{1}\left(\mathbb{R}^{n}, \mathbb{R}_{+}\right)$of a degree $l>0$ such that $\frac{\partial V}{\partial x} f(x)$ is $\mathbf{d}-$ homogeneous of the degree $l+\nu$, continuous and negative definite on $\mathbb{R}^{n}$.

The following corollary of the latter theorem is important for some further constructions.

Theorem 3.10 (Polyakov, 2018). Let $\mathbf{d}$ be a linear dilation on $\mathbb{R}^{n}$ and let $f: \mathbb{R}^{n} \backslash\{\mathbf{0}\} \rightarrow \mathbb{R}^{n}$ be a continuous $\mathbf{d}$-homogeneous vector field. Then the following claims are equivalent:

1) The origin of the system (3.8) is asymptotically stable.

2) The origin of the system

$$
\dot{z}=\|z\|_{P}^{1+\mu}\left(\frac{\left(I_{n}-G_{\mathbf{d}}\right) z^{\top} z P}{z^{\top} P G_{\mathbf{d}} z}+I_{n}\right) f\left(\frac{z}{\|z\|_{P}}\right)
$$

is asymptotically stable with $\|z\|_{P}=\sqrt{z^{\top} P z}$ and $P$ satisfying

$$
P G_{\mathbf{d}}+G_{\mathbf{d}}^{\top} P>0, \quad 0<P=P^{\top} \in \mathbb{R}^{n \times n},
$$

where $G_{\mathbf{d}} \in \mathbb{R}^{n \times n}$ is a generator of the dilation $\mathbf{d}$.

3) For any matrix $P \in \mathbb{R}^{n \times n}$ satisfying (3.10) there exists a d-homogeneous vector field $\Psi: \mathbb{R}^{n} \rightarrow \mathbb{R}^{n}$ of the degree $\mathbf{0}$ such that $\Psi \in C^{\infty}\left(\mathbb{R}^{n} \backslash\{\mathbf{0}\}, \mathbb{R}^{n}\right) \cap C\left(\mathbb{R}^{n}, \mathbb{R}\right)$ is diffemorphism on $\mathbb{R}^{n} \backslash\{\mathbf{0}\}$, homeomorphism on $\mathbb{R}^{n}, \Psi(\mathbf{0})=\mathbf{0}$ and

$$
\frac{\partial\left(\Psi^{\top}(x) P \Psi(x)\right)}{\partial x} f(x)<0 \quad \text { if } \quad \Psi^{\top}(x) P \Psi(x)=1 .
$$

Notice that the latter means that $\|\Psi\|_{\mathbf{d}} \in C^{\infty}\left(\mathbb{R}^{n} \backslash\{\mathbf{0}\}\right) \cap$ $C\left(\mathbb{R}^{n}, \mathbb{R}\right)$ is a $\mathbf{d}$-homogeneous Lyapunov function of the degree 1 for the system (3.8), where $\|\cdot\|_{\mathbf{d}}$ is the canonical homogeneous norm induced by $\|x\|_{P}=\sqrt{x^{\top} P x}$. 
4) For any matrix $P \in \mathbb{R}^{n \times n}$ satisfying (3.10) there exists a mapping

$$
\Xi \in C^{\infty}\left(\mathbb{R}^{n} \backslash\{\mathbf{0}\}, \mathbb{R}^{n \times n}\right)
$$

such that

$$
\begin{gathered}
\operatorname{det}(\Xi(z)) \neq 0, \quad \frac{\partial \Xi(z)}{\partial z_{i}} z=0, \quad \Xi\left(e^{s} z\right)=\Xi(z) \\
\text { for } z=\left(z_{1}, \ldots, z_{n}\right)^{\top} \in \mathbb{R}^{n} \backslash\{\mathbf{0}\}, s \in \mathbb{R}, i=1, \ldots, n \text { and } \\
z^{\top} \Xi^{\top}(z) P \Xi(z)\left(\frac{\left(I_{n}-G_{\mathbf{d}}\right) z z^{\top} P}{z^{\top} P G_{\mathbf{d}} z}+I_{n}\right) f\left(\frac{z}{\sqrt{z^{\top} P z}}\right)<0 .
\end{gathered}
$$

This theorem proves two important facts:

- Any d-homogeneous system is diffeomorphic on $\mathbb{R}^{n} \backslash\{\mathbf{0}\}$ to a standard homogeneous one.

- Any asymptotically stable $\mathbf{d}$-homogeneous system is homeomorphic on $\mathbb{R}^{n}$ and diffeomorphic on $\mathbb{R}^{n} \backslash\{\boldsymbol{0}\}$ to a quadratically stable one. Recall that an ODE system is said to be quadratically stable if it admits a quadratic Lyapunov function $V(z)=$ $z^{\top} P z, z \in \mathbb{R}^{n}, 0 \prec P=P^{\top} \in \mathbb{R}^{n \times n}$. Indeed, making the change of variables $\xi=\Psi(x)$ in (3.8) we derive

$$
\dot{\xi}=f_{\text {new }}(\xi),
$$

where

$$
\tilde{f}_{\text {new }}(\xi)=\left.\frac{\partial \Psi(x)}{\partial x} f(x)\right|_{x=\Psi^{-1}(\xi)} .
$$

The criterion (3.11) implies that $\xi^{\top} P \dot{\xi}<0$ if $\xi^{\top} P \xi=1$, i.e. the canonical homogeneous norm $\|\cdot\|_{\mathbf{d}}$ is the Lyapunov function of the latter system. Finally, the change of variable $z=$ $\|x\|_{\mathbf{d}} \mathbf{d}\left(-\ln \|x\|_{\mathbf{d}}\right) x$ gives $\|x\|_{\mathbf{d}}=\|z\|_{P}$, i.e., the transformed (homeomorphic) system

$$
\dot{z}=\tilde{f}(z):=\|z\|_{P}^{1+\mu}\left(\frac{\left(I_{n}-G_{\mathbf{d}}\right) z^{\top} z P}{z^{\top} P G_{\mathbf{d}} z}+I_{n}\right) f_{\text {new }}\left(\frac{z}{\|z\|_{P}}\right)
$$

is quadratically stable provided that the system (3.8) is asymptotically stable and vice versa. 


\subsection{Robustness}

Let us consider the following nonlinear system

$$
\dot{x}=f(x, q), \quad q \in L^{\infty}\left(\mathbb{R}_{+}, \mathbb{R}^{m}\right),
$$

where $x(t) \in \mathbb{R}^{n}$, as before, is a system state and $q(t) \in \mathbb{R}^{m}$ is a uniformly essentially bounded exogenous input. The ISS property Sontag, 2007 was defined in the previous section.

The following theorem was originally proven for standard and weighted dilations (see Ryan, 1995, Andrieu et al., 2008, Bernuau et al., 2013). It holds also for both linear and nonlinear geometric dilations (see Bernuau et al., 2014a):

Theorem 3.11. Let the vector field $\tilde{f}: \mathbb{R}^{n+m} \rightarrow \mathbb{R}^{n+m}$ given by

$$
\tilde{f}(x, q)=\left(\begin{array}{c}
f(x, q) \\
\mathbf{0}
\end{array}\right), \quad x \in \mathbb{R}^{n}, \quad q \in \mathbb{R}^{m}
$$

be continuous and $\mathbf{d}$-homogeneous of a degree $\mu \in \mathbb{R}$ with respect to a geometric dilation $\mathbf{d}(s)(x, q):=\left(\mathbf{d}_{1}(s) x, \mathbf{d}_{2}(s) q\right)$ in $\mathbb{R}^{n+m}$, where $\mathbf{d}_{1}$ and $\mathbf{d}_{2}$ are geometric dilations in $\mathbb{R}^{n}$ and $\mathbb{R}^{m}$, respectively. If the origin of the system

$$
\dot{x}(t)=f(x(t), \mathbf{0}), \quad x(t) \in \mathbb{R}^{m}
$$

is asymptotically stable then the system

$$
\dot{x}=f(x, q), \quad q \in L^{\infty}\left(\mathbb{R}_{+}, \mathbb{R}^{m}\right)
$$

is ISS.

Apart of robustness with respect to exogenous perturbations, the homogeneous systems also demonstrate a remarkable insensitivity feature with respect to the delays Zimenko et al., 2017. 


\subsection{Homogeneous approximations}

The class of geometric homogeneous systems is rather large. For example, any globally asymptotically stable at the origin system $\dot{x}=f(x)$ with $f \in C^{1}\left(\mathbb{R}^{n}, \mathbb{R}^{n}\right)$ is homogeneous of zero degree with respect to dilation generated by the Euler vector field $g(x)=-f(x)$, i.e., with respect to negation of itself. However, much more inclusive class of dynamical systems is composed by ones, which admit a local approximation by homogeneous models (Zubov, 1958; Hermes, 1991). Then advantage of having a homogeneous approximation is that stability properties of the approximation can be projected on the behavior of the original dynamics, and to analyze the stability of the approximating model all the methods of the theory of homogeneous systems can be used.

Following (Zubov, 1958; Andrieu et al., 2008), we may now define the local homogeneous approximations as follows.

Definition 3.11. Let $\phi, \eta: \mathbb{R}^{n} \rightarrow \mathbb{R}$ be functions and let $f, h: \mathbb{R}^{n} \rightarrow$ $\mathbb{R}^{n}$ be vector fields.

- The function $\eta$ is the $\mathbf{d}$-homogeneous approximation of degree $\kappa$ at 0 of the function $\phi$ if:

$$
\left|e^{-\kappa s} \phi(\mathbf{d}(s) x)-\eta(x)\right| \rightarrow 0 \text { as } s \rightarrow-\infty
$$

uniformly on $x$ from the unit sphere.

- The vector field $h$ is the $\mathbf{d}$-homogeneous approximation of degree $\kappa$ at 0 of the vector field $f$ if:

$$
\left\|e^{-\kappa s} \mathbf{d}(-s) f(\mathbf{d}(s) x)-h(x)\right\| \rightarrow 0 \text { as } s \rightarrow-\infty
$$

uniformly on $x$ from the unit sphere.

If the uniform convergence is taken when $s \rightarrow+\infty$, we get the approximation at $\infty$.

Similarly, local homogeneity can be defined for $s \rightarrow s^{\star}$ with any 
$s^{\star} \in[-\infty,+\infty]$ (Efimov and Perruquetti, 2016) (for a finite value of $s^{\star}$ the uniformity on the unit sphere is not needed).

It is worth highlighting that the class of systems having homogeneous approximations can be further extended to ones admitting homogeneous or sub-/sup-homogeneous extensions (Braidiz et al., 2019; Braidiz et al., 2020).

Results presented below in this section can be found in (Bernuau, 2013). And the first observation is that the homogeneous approximations are continuous.

Proposition 3.2. $\quad$ Let $\phi$ be a function. Assume that the $\mathbf{d}-$ homogeneous approximation of degree $\kappa$ at 0 or at $\infty$ of $\phi$ exists and is denoted by $\eta$. Then $\eta$ is a continuous $\mathbf{d}-$ homogeneous function of degree $\kappa$.

- Let $f$ be a vector field. Assume that the d-homogeneous approximation of degree $\kappa$ at 0 or at $\infty$ of $f$ exists and is denoted by $h$. Then $h$ is a continuous $\mathbf{d}$-homogeneous vector field of degree $\kappa$.

Let us show that qualitative properties of a vector field are encoded in its homogeneous approximations.

Theorem 3.12. Let a vector field $f$ have $\mathbf{d}$-homogeneous approximation $h_{0}$ at 0 with the degree $\kappa_{0}$. If the origin is an asymptotically stable equilibrium for $h_{0}$, then it is a locally asymptotically stable equilibrium for $f$.

Moreover, if the degree of the homogenization in negative, finite-time stability is achieved locally. 
Corollary 3.13. Under the assumptions of Theorem 3.12, if $\kappa_{0}<0$ then the origin is a locally FTS equilibrium of $f$.

A similar result exists for local approximation at $\infty$.

Theorem 3.14. Let a vector field $f$ have $\mathbf{d}$-homogeneous approximation $h_{\infty}$ at $\infty$ with the degree $\kappa^{\infty}$. If the origin is an asymptotically stable equilibrium of $h_{\infty}$, then $\dot{x}=f(x)$ has a globally finite-time stable strictly positive invariant compact neighborhood of the origin.

Moreover, if the degree of the homogenization is positive, the convergence to this compact set is achieved in a time, which is uniform in the initial condition.

Corollary 3.15. Under the assumptions of Theorem 3.14, if $\kappa^{\infty}>0$ then $\dot{x}=f(x)$ has a globally fixed-time stable strictly positive invariant compact neighborhood of the origin.

Existence of homogeneous approximations at 0 and $\infty$ with proper degrees results in a uniform attractivity of the origin.

Corollary 3.16. If the origin of $f$ is globally asymptotically stable and $f$ satisfies the conditions of both corollaries, 3.13 and 3.15, then the origin of $f$ is globally fixed-time stable.

The simplest example of locally homogeneous system is considered in Section 1.3.2. 
Example 3.1. To illustrate these results consider a simple example:

$$
\dot{x}=\sum_{i=1}^{N} a_{i} x^{\alpha_{i}},
$$

where $x, a_{1}, \ldots, a_{N} \in \mathbb{R}, 0<\alpha_{1}<\alpha_{2}<\cdots<\alpha_{N}<+\infty$ and an integer $N \geq 1$. Taking the standard dilation, i.e., $\mathbf{d}(s)=e^{s}$, for $f(x)=$ $\sum_{i=1}^{N} a_{i} x^{\alpha_{i}}$ and choosing for approximation at zero $h_{0}(x)=a_{1} x^{\alpha_{1}}$ with degree $\kappa_{0}=\alpha_{1}-1$ and for approximation at infinity $h_{\infty}(x)=a_{N} x^{\alpha_{N}}$ with $\kappa_{\infty}=\alpha_{N}-1$ we validate Definition 3.11:

$$
\begin{gathered}
\limsup _{s \rightarrow-\infty}\left\|e^{-\kappa_{0} s} \mathbf{d}(-s) f(\mathbf{d}(s) x)-h_{0}(x)\right\| \\
=\limsup _{s \rightarrow-\infty}\left\|e^{-\left(\alpha_{1}-1\right) s} e^{-s} \sum_{i=1}^{N} a_{i} e^{\alpha_{i} s} x^{\alpha_{i}}-a_{1} x^{\alpha_{1}}\right\| \\
=\limsup _{s \rightarrow-\infty}\left\|\sum_{i=2}^{N} a_{i} e^{\left(\alpha_{i}-\alpha_{1}\right) s} x^{\alpha_{i}}\right\|=0
\end{gathered}
$$

since $\alpha_{i}-\alpha_{1}>0$ for all $i=2, \ldots, N$, and

$$
\begin{gathered}
\limsup _{s \rightarrow+\infty}\left\|e^{-\kappa_{\infty} s} \mathbf{d}(-s) f(\mathbf{d}(s) x)-h_{\infty}(x)\right\| \\
=\limsup _{s \rightarrow+\infty}\left\|e^{-\left(\alpha_{N}-1\right) s} e^{-s} \sum_{i=1}^{N} a_{i} e^{\alpha_{i} s} x^{\alpha_{i}}-a_{N} x^{\alpha_{N}}\right\| \\
=\limsup _{s \rightarrow+\infty}\left\|\sum_{i=1}^{N-1} a_{i} e^{\left(\alpha_{i}-\alpha_{N}\right) s} x^{\alpha_{i}}\right\|=0
\end{gathered}
$$

since $\alpha_{i}-\alpha_{N}<0$ for all $i=1, \ldots, N-1$, respectively. Therefore, $h_{0}$ and $h_{\infty}$ are indeed homogeneous approximations for $f$, and the systems

$$
\dot{x}=a_{j} x^{\alpha_{j}}, j \in\{1, N\}
$$

are local approximating dynamics for (3.16). Hence, this system is locally asymptotically stable at the origin if $a_{1}<0$ and globally asymptotically/finite-time stable with respect to a ball containing the origin if $a_{N}<0$ according to theorems 3.12 and 3.14, respectively. Under these restrictions, the system (3.16) demonstrates a finite-time convergence rate if $\alpha_{1}<1$ or a uniform convergence time to a ball if 
$\alpha_{N}>1$ due to corollaries 3.13 and 3.15, respectively. Note that under the hypotheses of theorems 3.12 and 3.14, in general case, we cannot make a conclusion about global stability properties of the system (3.16) due to undefined signs of the gains $a_{i}$ with $i=2, \ldots, N-1$. However, if $N=2$, and the conditions of corollaries 3.13 and 3.15 are verified, hence it is the case of Corollary 3.16, then the system (3.16) is globally fixed-time stable.

As we have shown, asymptotic stability of a homogeneous system implies its robustness (in ISS or iISS sense) against properly located exogenous inputs, then from (i)ISS of the homogeneous approximations the respective conclusions can be done about robustness of the original dynamics (Bernuau et al., 2013).

\subsection{Homogeneity in Hilbert spaces}

The linear geometric homogeneity of evolution systems in Banach and Hilbert spaces can be developed similarly to the finite dimensional case using the strongly continuous groups of linear bounded operators.

Note that, for time-delay systems there is another way to define homogeneity (Efimov et al., 2014a; Efimov et al., 2016), which can be similarly extended to time-varying dynamics (Ríos et al., 2016; Ríos et $a l ., 2017)$. However, the development of homogeneity theory to discretetime models requires a complete revisiting of all procedures (Sanchez et al., 2019; Sanchez et al., 2020).

\section{Dilations in abstract spaces}

Let $\mathcal{L}:=\mathcal{L}(\mathbb{H}, \mathbb{H})$ denote the space of linear bounded operators $\mathbb{H} \rightarrow \mathbb{H}$, where $\mathbb{H}$ is a real Hilbert space with the norm $\|\cdot\|=\sqrt{\langle\cdot, \cdot\rangle}$ by an inner product. A topological characterization of a dilations in an abstract space can be found in Husch, 1970. In this paper, we deal only with the so-called linear dilations in Hilbert spaces (Polyakov et al., 2016a; Polyakov et al., 2018). 
Definition 3.12. A mapping $\mathbf{d}: \mathbb{R} \rightarrow \mathcal{L}$ is said to be a linear dilation in $\mathbb{H}$ if

- (Group property)

$$
\mathbf{d}(0)=I \in \mathcal{L} \text { and } \mathbf{d}(t+s)=\mathbf{d}(t) \mathbf{d}(s)=\mathbf{d}(s) \mathbf{d}(t) \text { for } t, s \in \mathbb{R} ;
$$

- (Limit property)

$$
\lim _{s \rightarrow-\infty}\|\mathbf{d}(s)\|=0 \text { and } \lim _{s \rightarrow+\infty}\|\mathbf{d}(s)\|=\infty
$$

Obviously that $\mathbf{d}$ is a group of linear bounded invertible operators

$$
\mathbf{d}(-s)=(\mathbf{d}(s))^{-1} .
$$

The limit property specifies groups being dilations in normed spaces.

Recall (see Pazy, 1983, page 4) that a dilation $\mathbf{d}$ is

- strongly continuous if $\mathbf{d}(\cdot) z: \mathbb{R} \rightarrow \mathbb{H}$ is continuous for any $z \in \mathbb{H}$

- uniformly continuous if $\mathbf{d}(\cdot): \mathbb{R} \rightarrow \mathcal{L}$ is continuous in the operator norm.

For $\mathbb{H}=\mathbb{R}^{n}$ there is no reason to deal with two types of continuity, since any strongly continuous group of linear bounded operators in a finite-dimensional space is uniformly continuous. Dilations in $\mathbb{H}=\mathbb{R}^{n}$ we studied in the previous subsections.

Lemma 3.17 (Polyakov, 2020). Let the operator $\mathbf{d}(s)$ be defined as

$$
(\mathbf{d}(s) z)(y)=e^{s G_{\alpha}} z\left(e^{s G_{\beta}} y\right), \quad G_{\alpha} \in \mathbb{R}^{m \times m}, \quad G_{\beta} \in \mathbb{R}^{n \times n},
$$

where $s \in \mathbb{R}$ is a parameter, $z: \mathbb{R}^{n} \rightarrow \mathbb{R}^{m}, y \in \mathbb{R}^{n}$. Then

- $\mathbf{d}$ is a group of linear invertible operators on $C_{c}^{\infty}\left(\mathbb{R}^{n}, \mathbb{R}^{m}\right)$, $C_{0}^{\infty}\left(\mathbb{R}^{n}, \mathbb{R}^{m}\right)$ 
- $\mathbf{d}$ is a group of linear bounded invertible operators on $L^{p}\left(\mathbb{R}^{n}, \mathbb{R}^{m}\right)$ and

$$
\|\mathbf{d}(s) z\|_{p}=e^{-s \operatorname{tr}\left(G_{\beta}\right) / p}\left\|e^{s G_{\alpha}} z\right\|_{p},
$$

where $\|z\|_{p}:=\left(\int_{\mathbb{R}^{n}}|z(y)|^{p} d y\right)^{1 / p}$;

- $\mathbf{d}$ is a dilation on $L^{p}\left(\mathbb{R}^{n}, \mathbb{R}^{m}\right)$ provided that the matrix

$$
G_{\alpha}-\frac{\operatorname{tr}\left(G_{\beta}\right)}{p} I_{m} \in \mathbb{R}^{m \times m}
$$

is anti-Hurwitz.

- $\mathbf{d}$ is a strongly continuous group in $L^{2}\left(\mathbb{R}^{n}, \mathbb{R}^{m}\right)$.

Generators of dilations groups play an important role for analysis and design of homogeneous control systems.

Definition 3.13 (Pazy, 1983). An operator $G_{\mathbf{d}}: \mathcal{D}\left(G_{\mathbf{d}}\right) \subset \mathbb{H} \rightarrow \mathbb{H}$ defined as

$$
G_{\mathbf{d}} z=\lim _{s \rightarrow 0^{+}} \frac{\mathbf{d}(s) z-z}{s}
$$

is called an infinitesimal generator of a strongly continuous group d. The domain of the generator $G_{\mathbf{d}}$ is given by

$$
\mathcal{D}\left(G_{\mathbf{d}}\right)=\left\{z \in \mathbb{H}: \lim _{s \rightarrow 0^{+}} \frac{\mathbf{d}(s) z-z}{s} \text { exists }\right\} .
$$

The next lemma specifies a generator of the dilation (3.17) in $L^{2}$.

Lemma 3.18 (Polyakov, 2020). Let a group of linear bounded operators $\mathbf{d}$ in $L^{2}\left(\mathbb{R}^{n}, \mathbb{R}^{m}\right)$ be given by (3.17). Then the generator of $\mathbf{d}$ is defined as follows

$$
\left(G_{\mathbf{d}} z\right)(y)=G_{\alpha} z(y)+\left(G_{\beta} y\right) \cdot \nabla z(y), \quad y \in \mathbb{R}^{n}, G_{\alpha} \in \mathbb{R}^{m \times m}, G_{\beta} \in \mathbb{R}^{n \times n}
$$


with the domain

$$
\mathcal{D}\left(G_{\mathbf{d}}\right)=\left\{z \in L^{2}\left(\mathbb{R}^{n}, \mathbb{R}^{m}\right): G_{\alpha} z+\left(G_{\beta} y\right) \cdot \nabla z \in L^{2}\left(\mathbb{R}^{n}, \mathbb{R}^{m}\right)\right\} .
$$

Moreover,

$$
\left\langle G_{\mathbf{d}} z, z\right\rangle=\left\langle\left(G_{\alpha}-0.5 \operatorname{tr}\left(G_{\beta}\right) I_{m}\right) z, z\right\rangle
$$

for any $z \in \mathcal{D}\left(G_{\mathbf{d}}\right)$.

A dilation in $\mathbb{H}$ introduces a new norm-topology on $\mathbb{H}$ by means of the so-called canonical homogeneous norm (studied below) with d-homogeneous spheres

$$
S_{\mathbf{d}}\left(r, z_{0}\right)=\left\{z \in \mathbb{H}:\left\|\mathbf{d}(-\ln (r))\left(z-z_{0}\right)\right\|=1\right\}
$$

and balls

$$
B_{\mathbf{d}}\left(r, z_{0}\right)=\left\{z \in \mathbb{H}:\left\|\mathbf{d}(-\ln (r))\left(z-z_{0}\right)\right\|<1\right\},
$$

where $r>0$ denotes the radius and $z_{0} \in \mathbb{H}$ is a center. The notations $S_{\mathbf{d}}(r)$ and $B_{\mathbf{d}}(r)$ are utilized for $z_{0}=\mathbf{0 .}$

Definition 3.14 (Polyakov et al., 2018). A nonempty set $\mathcal{D} \subseteq \mathbb{H}$ is said to be a $\mathbf{d}$-homogeneous cone in $\mathbb{H}$ if $\mathcal{D}$ is invariant with respect to a dilation $\mathbf{d}$ :

$$
\mathbf{d}(s) z \in \mathcal{D} \text { for } z \in \mathcal{D} \text { and } s \in \mathbb{R} \text {. }
$$

Differential operators in the space $L^{2}\left(\mathbb{R}^{n}, \mathbb{R}^{m}\right)$ are defined on $\mathbf{d}$ homogeneous cones (see examples below).

Monotonicity of linear dilations in a Hilbert space can be introduced similarly to the Definition 3.8.

Definition 3.15 (Polyakov et al., 2018). A linear dilation $\mathbf{d}$ in $\mathbb{H}$ is strictly monotone if there exists $\beta>0$ such that $\sup _{\|x\|=1}\|\mathbf{d}(s) x\| \leq$ $e^{\beta s}, \forall s \leq 0$. 
Monotone dilations have several important properties.

Proposition 3.3 (Polyakov et al., 2018). If $\mathbf{d}$ is a strongly continuous strictly monotone dilation then

1) the homogeneous projection is unique, i.e. for any $u \in \mathbb{H}$ there exists a unique pair $\left(s_{0}, u_{0}\right) \in \mathbb{R} \times S$ such that $u=\mathbf{d}\left(s_{0}\right) u_{0}$;

2) the function $\|\mathbf{d}(\cdot) u\|: \mathbb{R} \rightarrow \mathbb{R}_{+}$(with $u \in \mathbb{H} \backslash\{\mathbf{0}\}$ ) is continuous and strictly increasing on $\mathbb{R}$;

3) $\|\mathbf{d}(s) u\|>1$ for all $s>0$ and all $u \in S$.

In the Hilbert space monotone dilations characterized by means of the following lemma.

Proposition 3.4 (Polyakov, 2020). A strongly continuous dilation group $\mathbf{d}$ in a real Hilbert space $\mathbb{H}$ is strictly monotone if and only if there exists $\beta>0$ and a $\mathbf{d}$-homogeneous cone $\mathcal{D}$ dense in $\mathcal{D}\left(G_{\mathbf{d}}\right)$ such that

$$
\left\langle G_{\mathbf{d}} z, z\right\rangle \geq \beta \quad \text { for any } \quad z \in S \cap \mathcal{D},
$$

where $G_{\mathbf{d}}$ is the generator of $\mathbf{d}$.

Similarly to $\mathbb{R}^{n}$, it can be shown that any dilation in $\mathbb{H}$ is strictly monotone provided that a inner product in $\mathbb{H}$ is properly selected.

\section{Canonical homogeneous norm in $\mathbb{H}$}

The canonical homogeneous norm considered above in $\mathbb{R}^{n}$ was originally introduced for Banach and Hilbert spaces (see Polyakov et al., 2018). 
The functional $\|\cdot\|_{\mathbf{d}}: \mathbb{H} \rightarrow[0,+\infty)$ defined as

$$
\|u\|_{\mathbf{d}}=e^{s_{u}}, \text { where } s_{u} \in \mathbb{R}:\left\|\mathbf{d}\left(-s_{u}\right) u\right\|=1,
$$

is called the canonical homogeneous norm in $\mathbb{H}$, where $\mathbf{d}$ is a strictly monotone strongly continuous dilation on $\mathbb{H}$.

The canonical homogeneous norm is well-defined (single-valued and continuous) on $\mathbb{H}$ and $\|\mathbf{0}\|_{\mathbf{d}}=0$ by continuity.

Lemma 3.19 (Polyakov et al., 2018). If $\mathbf{d}$ is a strictly monotone strongly continuous dilation on $\mathbb{H}$ then

- there exist constants $\alpha>\beta>0$ and $M \geq 1$ such that

$$
\frac{1}{M}\|u\|_{\mathbf{d}}^{\alpha} \leq\|u\| \leq\|u\|_{\mathbf{d}}^{\beta}, \quad u \in B_{\mathbf{d}}(1)
$$

and

$$
\|u\|_{\mathbf{d}}^{\beta} \leq\|u\| \leq M\|u\|_{\mathbf{d}}^{\alpha}, \quad u \in \mathbb{H} \backslash B_{\mathbf{d}}(1) ;
$$

- $\|\cdot\|_{\mathbf{d}}$ is locally Lipschitz continuous on $\mathbb{H} \backslash\{\mathbf{0}\}$ and differentiable on $\mathcal{D}\left(G_{\mathbf{d}}\right) \backslash\{\mathbf{0}\}$ with the Fréchet derivative of $\|\cdot\|_{\mathbf{d}}$ at $u \in \mathcal{D}\left(G_{\mathbf{d}}\right) \backslash\{\mathbf{0}\}$ given by

$$
\left(D\|u\|_{\mathbf{d}}\right)(\cdot)=\frac{\left\langle\mathbf{d}\left(-\ln \|u\|_{\mathbf{d}}\right) \cdot, \mathbf{d}\left(-\ln \|u\|_{\mathbf{d}}\right) u\right\rangle}{\left\langle G_{\mathbf{d}} \mathbf{d}\left(-\ln \|u\|_{\mathbf{d}}\right) u, \mathbf{d}\left(-\ln \|u\|_{\mathbf{d}}\right) u\right\rangle}\|u\|_{\mathbf{d}} .
$$

Homogeneous norm is not a norm in the general case, since the triangle inequality does not hold. However, it is a norm in a Banach space homeomorphic to $\mathbb{H}$.

Let us denote

$$
\Phi(x)=\|x\|_{\mathbf{d}} \mathbf{d}\left(-\ln \|x\|_{\mathbf{d}}\right) x, \quad x \in \mathbb{H} .
$$

One can be shown that $\Phi$ is homeomorphism on $\mathbb{H}$ and its inverse is 
given by

$$
\Phi^{-1}(z)=\|z\|^{-1} \mathbf{d}(\ln \|z\|) z, \quad z \in \mathbb{H} .
$$

Theorem 3.20 (Polyakov, 2020). Let $\mathbf{d}$ be a strongly continuous strictly monotone dilation in a real Hilbert space $\mathbb{H}$. Let an addition operation $\tilde{+}: \mathbb{H} \times \mathbb{H} \rightarrow \mathbb{H}$ and a multiplication by a scalar $\tilde{~: ~}$ $\mathbb{R} \times \mathbb{H} \rightarrow \mathbb{H}$ be defined as follows

- $x \tilde{+} y:=\Phi^{-1}(\Phi(x)+\Phi(y))$, where $x, y \in \mathbb{H}$,

- $\lambda \approx x:=\operatorname{sign}(\lambda) \mathbf{d}(\ln |\lambda|) x$, where $\lambda \in \mathbb{R}, x \in \mathbb{H}$.

Then the set $\mathbb{H}$ together with the operations $\tilde{+}$ and $\tilde{r}$ is a linear normed vector space $\tilde{\mathbb{H}}$ with the norm given by $\|\cdot\|_{\mathbf{d}}$. Moreover, $\tilde{\mathbb{H}}$ is a real Banach space.

The theorem justifies the name "norm" for the functional $\|\cdot\|_{\mathbf{d}}$, which now can be utilized for a convergence analysis in $\mathbb{H}$ on a pair with $\|\cdot\|$.

\section{Homogeneous functionals and operators}

Definition 3.16 (Polyakov et al., 2016a). A functional $h: \mathcal{D}(h) \subset$ $\mathbb{H} \rightarrow \mathbb{R}$ is said to be d-homogeneous of a degree $\nu \in \mathbb{R}$ if its domain $\mathcal{D}(h)$ is a $\mathbf{d}$-homogeneous cone and

$$
h(\mathbf{d}(s) u)=e^{\nu s} h(u) \quad \text { for } \quad s \in \mathbb{R}, \quad u \in \mathcal{D}(h),
$$

where $\mathbf{d}$ is a group of linear bounded invertible operators in $\mathbb{H}$.

The homogeneous norm studied in the previous section is an example of the so-called homogeneous functional. 
Definition 3.17 (Polyakov et al., 2016a). An operator $f: \mathcal{D}(f) \subset$ $\mathbb{H} \rightarrow \mathbb{H}$ is said to be $\mathbf{d}$-homogeneous of a degree $\nu \in \mathbb{R}$ if its domain $\mathcal{D}(f)$ is a d-homogeneous cone and

$$
f(\mathbf{d}(s) u)=e^{\nu s} \mathbf{d}(s) f(u) \quad \text { for } \quad s \in \mathbb{R}, \quad u \in \mathcal{D}(f),
$$

where $\mathbf{d}$ is a group of linear bounded operators in $\mathbb{H}$.

Notice that the identity (3.24) can be understood in the week sense as shown in the following example.

Example 3.2 (Polyakov, 2020). Let us consider the Laplace operator

$$
\Delta: \mathcal{D}(\Delta) \subset L^{2} \rightarrow L^{2},
$$

with the domain

$$
\mathcal{D}(A)=\left\{u \in L^{2}: \exists f \in L_{l o c}^{1} \text { such that } \int u \cdot \Delta \phi=\int f \cdot \phi, \quad \forall \phi \in C_{c}^{\infty}\right\},
$$

where, for shortness, we denote $L^{2}=L^{2}\left(\mathbb{R}^{n}, \mathbb{R}^{m}\right)$ in this example.

Let us show that the operator $\Delta$ is $\mathbf{d}$-homogeneous of degree $2 \beta$ provided that the dilation $\mathbf{d}$ is given by (3.17) with $G_{\alpha}=\alpha I_{m}, G_{\beta}=$ $\beta I_{n}$.

By Lemma 3.17, $\mathbf{d}$ is a group of linear invertible operators on $C_{c}^{\infty}$ and, consequently, $\mathbf{d}(s)$ maps $C_{c}^{\infty}$ onto $C_{c}^{\infty}$. Notice that if $\phi \in C_{c}^{\infty}$ then, obviously,

$$
(\Delta \circ \mathbf{d}(s)) \phi)(x)=e^{(\alpha+2 \beta) s}(\Delta \phi)\left(e^{\beta s} x\right)=e^{2 \beta s}((\mathbf{d}(s) \circ \Delta) \phi)(x)
$$

for all $s \in \mathbb{R}, x \in \mathbb{R}^{n}$. In other words, the Laplace operator is dhomogeneous as an operator $C_{c}^{\infty} \rightarrow C_{c}^{\infty}$. Since $C_{c}^{\infty}$ is dense in $L^{2}$, $H^{1}$ and $H^{2}$ then, it is expectable, the $\Delta$ is $\mathbf{d}$-homogeneous as an operator in $\mathcal{D}(\Delta) \subset L^{2} \rightarrow L^{2}$ as well. Let us prove this claim rigorously.

Let $u \in \mathcal{D}(\Delta)$ and $\Delta u=f \in L_{l o c}^{1}$ in the weak sense, i.e.,

$$
\int_{\mathbb{R}^{n}} u \Delta \phi=\int_{\mathbb{R}^{n}} f \phi, \quad \forall \phi \in C_{c}^{\infty} .
$$


Since $\mathbf{d}(s) f \in L_{l o c}^{1}$ then using the change-of-variable theorem (see ,e.g., Netuka, 2011) in the Lebesgue integral we derive

$$
\begin{gathered}
e^{2 \beta s} \int(\mathbf{d}(s) f) \cdot \phi=e^{(\alpha+2 \beta) s} \int f\left(e^{\beta s} x\right) \cdot \phi(x) d x \\
=e^{(\alpha+(2-n) \beta) s} \int f(x) \cdot \phi\left(e^{-\beta s} x\right) d x=e^{(2 \alpha+(2-n) \beta) s} \int f \cdot \tilde{\phi} \\
=e^{(2 \alpha+(2-n) \beta) s} \int u \cdot \Delta \tilde{\phi}=e^{(2 \alpha+(2-n) \beta) s} \int u \cdot(\Delta \circ \mathbf{d}(-s)) \phi \\
=e^{(\alpha-n \beta) s} \int u(x) \cdot \Delta \phi\left(e^{-\beta s} x\right) d x=e^{\alpha s} \int u\left(e^{\beta s} x\right) \cdot \Delta \phi(x) d x \\
=\int(\mathbf{d}(s) u) \cdot \Delta \phi,
\end{gathered}
$$

where $\tilde{\phi}=\mathbf{d}(-s) \phi \in C_{c}^{\infty}$. Hence, $\mathbf{d}(s) u \in \mathcal{D}(\Delta)$ and $(\Delta \circ \mathbf{d}(s)) u=$ $e^{2 \beta s} \mathbf{d}(s) f=e^{2 \beta s}(\mathbf{d}(s) \circ \Delta) u$ in the weak sense for any $s \in \mathbb{R}$ and any $u \in \mathcal{D}(\Delta)$.

In the similar way one can be shown that most of nonlinear models of mathematical physics (like Burgers, Navier-Stokes, KdV, SaintVenant equations) are $\mathbf{d}$-homogeneous in the considered generalized sense. For more results about homogeneous differential operators and homogeneous Lie groups we refer the reader to Folland, 1975 and Fischer and Ruzhanky, 2016.

\subsubsection{Homogeneous evolution equations}

Let us consider the nonlinear system (evolution equation)

$$
\dot{x}=A x+f(x), \quad t>0,
$$

where $x(t) \in \mathbb{H}$ is the system state, $A: \mathcal{D}(A) \subset \mathbb{H} \rightarrow \mathbb{H}$ is a linear (possibly) unbounded closed densely defined operator which generates a strongly continuous semi-group $\Phi$ of linear bounded operators on a real Hilbert space $\mathbb{H}$ (see, e.g., Pazy, 1983, Chapter 1) and $f: \mathbb{H} \rightarrow \mathbb{H}$ is a non-linear mapping such that $f(\mathbf{0})=\mathbf{0}$. The latter means that the evolution equation always has the zero solution.

If $A$ is a bounded operator then $\Phi(t-\tau)=e^{(t-\tau) A}=\sum_{i=0}^{\infty} \frac{(t-\tau)^{i} A^{i}}{i !}$ is a uniformly continuous (i.e. continuous in the operator norm) semigroup 
of linear bounded operators on $\mathbb{H}$ and the latter series converges in the operator topology.

Definition 3.18. A continuous function $x:[0, T) \rightarrow \mathbb{H}$ is said to be a mild solution to (3.25) if

$$
x(t)=\Phi(t) x_{0}+\int_{0}^{t} \Phi(t-\tau) f(x(\tau)) d \tau, \quad \forall t \in[0, T) .
$$

If this mild solution satisfies (3.25) for (almost) all $t \in(0, T)$ and and $x(t) \in \mathcal{D}(A)$ ( resp. $x(t) \stackrel{\text { a.e. }}{\in} \mathcal{D}(A))$ then $x$ is called classical (resp. strong) solution of (3.25).

The integral in the above definition is understood in the sense of Bochner (see ,e.g., Driver, 2003, page 187).

The problem of existence of solutions for evolution models like (3.25) is studied in literature (see, e.g., Pazy, 1983; Engel and Nagel, 2000). Notice that if $\mathbb{H}=\mathbb{R}^{n}$ then, to guarantee existence of solutions, the operator $A$ must be bounded and, without lost of generality, we may assume $A=\mathbf{0}$, i.e. (3.25) becomes an ordinary differential equation.

Theorem 3.21. Let $A$ be a generator of a strongly continuous semigroup of linear bounded operators on a Hilbert space $\mathbb{H}$. If $f: \mathbb{R} \times \mathbb{H} \rightarrow \mathbb{H}$ is locally Lipschitz continuous on $\mathbb{R} \times(\mathbb{H} \backslash\{\mathbf{0}\})$ then the system (3.25) has a unique mild (a locally Lipschitz continuous strong) solution $x \in C\left(\left[t_{0}, t_{1}\right), \mathbb{H}\right)$ for any $x(0) \in \mathbb{H} \backslash\{\mathbf{0}\}$ (resp. $x(0) \in \mathcal{D}(A) \backslash\{\mathbf{0}\})$, where $t_{1}=+\infty$ or $t_{1}<+\infty: \lim _{t \rightarrow t_{1}}\|x(t)\|=$ $+\infty$ (or 0 ). Moreover, any mild solution of the system (3.25) with $x\left(t_{0}\right)=x_{0} \in \mathbb{H} \backslash\{\mathbf{0}\}$ is a uniform limit (on compacts intervals of time) of strong solutions with $x\left(t_{0}\right)=x_{i} \in \mathcal{D}(A)$, such that $x_{i} \rightarrow x_{0} \in \mathbb{H}$ as $i \rightarrow+\infty$.

The proof of the theorem for $f$ Lipschitz continuous on $\mathbb{R} \times \mathbb{H}$ can be found in Pazy, 1983, page 184. The case of the local Lipschitz continuity 
on $\mathbb{R} \times(\mathbb{H} \backslash\{\mathbf{0}\})$ can be treated considering $f_{\sigma}(t, x)=a_{\sigma}(\|x\|) f(t, x)$, where $a$ is the so-called "cut-off" function: $a \in C^{\infty}\left(\mathbb{R}_{+}, \mathbb{R}_{+}\right)$such that $a(\rho)=1$ for $\rho \in[2 \delta, 1 /(2 \delta)], \delta \in(0,1 / 2)$ and $a(\rho)=0$ for $\rho \notin[2 \delta, 1 /(2 \delta)]$. Since $f_{\sigma}$ is Lipschitz continuous on $\mathbb{R} \times \mathbb{H}$ then the system (3.25) with $f$ replaced by $f_{\sigma}$ has solutions on $\left[t_{0},+\infty\right)$ and tending $\delta \rightarrow 0$ we prove the claim. Finally, uniform convergence of strong solutions to mild solutions comes from continuous dependence of mild solutions on the initial data (see, Pazy, 1983, page 184). Examples PDEs admitting the representation (3.25) can be found in the literature, see, e.g. Tucsnak and Weiss, 2009, Engel and Nagel, 2000. Notice that the operator $f$ can be unbounded in the general case.

Example 3.3. Let us consider the nonlinear partial differential equation

$$
\frac{\partial x}{\partial t}=\Delta x+f(x)
$$

where $x(t, z) \in \mathbb{R}^{n}, z \in \mathbb{R}^{n}$ and $\Delta=\nabla \cdot \nabla$ is the Laplace operator. The system can be represented in the form (3.25) with $\mathbb{H}=L^{2}\left(\mathbb{R}^{n}, \mathbb{R}^{n}\right)$, $A=\Delta, \mathcal{D}(A)=H^{2}\left(\mathbb{R}^{n}, \mathbb{R}^{n}\right)$. A semigroup $\Phi$ generated by $\Delta$ is given by (see ,e.g., Engel and Nagel, 2000, page 69)

$$
(\Phi(t) x)(z)=\frac{1}{\sqrt{(4 \pi t)^{n}}} \int_{\mathbb{R}^{n}} e^{-\frac{\|z-y\|^{2}}{4 t}} x(y) d y, \quad z \in \mathbb{R}^{n} .
$$

The operator $f: \mathbb{H} \rightarrow \mathbb{H}$ defined as $f(x)=-\|x\|^{\gamma} x, \gamma \geq-1$ is, obviously, locally Lipschitz continuous on $\mathbb{H} \backslash\{\mathbf{0}\}$, so the system has mild solutions in this case. To prove the existence of mild solutions of (3.26) with $f: H^{1}\left(\mathbb{R}^{n}, \mathbb{R}^{n}\right) \subset \mathbb{H} \rightarrow \mathbb{H}$ given by

$$
f(x)=(x \cdot \nabla) x
$$

some more difficult constructions are needed (Pazy, 1983, page 238).

Example 3.4. Let us consider the functional differential equation

$$
\dot{y}(t)=g\left(t, y_{h}(t)\right), \quad t>0
$$

that is equivalent to the integral equation

$$
y(t)=y(0)+\int_{0}^{t} g\left(s, y_{h}(s)\right) d s,
$$


where $y(t) \in \mathbb{R}^{n}$ and $y_{h}(t) \in L^{2}\left((-h, 0), \mathbb{R}^{n}\right)$ is defined as follows $\left(y_{h}(t)\right)(\theta)=y(t+\theta), \theta \in[-h, 0], g:(0,+\infty) \times L^{2}\left((-h, 0), \mathbb{R}^{n}\right) \rightarrow \mathbb{R}^{n}$ is a non-linear operator that satisfies in $L^{2}\left([-h, 0], \mathbb{R}^{n}\right)$ the Lipschitz condition uniformly on $t$. The initial condition of the latter system is given by

$$
y(t)=y^{0}(t) \text { for } t \in[-h, 0] \quad\left(\text { i.e. } y_{h}(0)=y^{0} \in L^{2}\left((-h, 0), \mathbb{R}^{n}\right)\right) .
$$

Let us also consider the non-linear evolution system (3.25) in the Hilbert space

$$
\mathbb{H}=\mathbb{R} \times L^{2}\left([-h, 0], \mathbb{R}^{n}\right)
$$

with the operator $A=\left(\begin{array}{cc}\mathbf{0} & \mathbf{0} \\ \mathbf{0} & \frac{\partial}{\partial z}\end{array}\right)$ having the domain

$$
\mathcal{D}(A)=\left\{x=\left(x_{1}, x_{2}\right) \in \mathbb{H}: x_{2} \in H^{1}\left([-h, 0], \mathbb{R}^{n}\right) \text { and } x_{1}=x_{2}(0)\right\},
$$

and $f(t, x)=\left(\begin{array}{ll}g\left(t, x_{2}\right) & 0\end{array}\right)$. The operator $A$ generates the strongly continuous semigroup of linear bounded operators in $\mathbb{H}$ given by

$$
\begin{aligned}
& (\Phi(t) x)(z)=\left\{\begin{array}{ccc}
\left(x_{1}, x_{2}(t+z)\right) & \text { if } & -h \leq t+z \leq 0, \\
\left(x_{1}, x_{1}\right) & \text { if } & t+z>0,
\end{array}\right. \\
& t \geq 0, \quad z \in[-h, 0], \quad x=\left(x_{1}, x_{2}\right) \in \mathbb{H} .
\end{aligned}
$$

From Theorem 3.21 we conclude that the evolution system has a mild solution for any $u(0) \in \mathbb{H}$, in particular, for $u(0)=\left(x^{0}(0), x^{0}\right)$. Hence, we derive

$$
\left(x_{2}(t)\right)(z)=\left(\Phi(t) x_{0}\right)(z)+\int_{0}^{t} g\left(s, x_{2}(s)\right) d s, \quad z \in(-h, 0)
$$

and for $z \rightarrow 0$ the latter coincides with (3.27). Thus the original functional differential equation has a solution. Taking into account a continuity of the function $t \rightarrow \int_{0}^{t} g\left(s, x_{2}(s)\right) d s$ we conclude that the solution is continuous for $t>0$.

Lemma 3.22 (Polyakov, 2020). Let a linear closed densely defined operator $A: \mathcal{D}(A) \subset \mathbb{H} \rightarrow \mathbb{H}$ generate a strongly continuous semigroup $\Phi$ of linear bounded operators on $\mathbb{H}$ and $\mathbf{d}$ be a group of linear bounded invertible operators on $\mathbb{H}$. If the operator $A$ is 
d-homogeneous of a degree $\mu \in \mathbb{R}$ then

$$
\Phi(t) \mathbf{d}(s)=\mathbf{d}(s) \Phi\left(e^{\mu s} t\right), \quad \forall t \geq 0, \quad \forall s \in \mathbb{R} .
$$

Example 3.5. The Laplace operator $\Delta: H^{2} \subset L^{2} \rightarrow L^{2}$ is proven to be d-homogeneous of th degree $2 \beta$ with respect to the dilation $(\mathbf{d}(s) x)(z)=$ $e^{\alpha s} x\left(e^{\beta s} z\right), z \in \mathbb{R}^{n}, x \in L^{2}\left(\mathbb{R}^{n}, \mathbb{R}^{m}\right)$. The strongly continuous semigroup $\Phi$ generated by $\Delta$ is given by (see Example 3.2)

$$
(\Phi(t) x)(z)=\frac{1}{\sqrt{(4 \pi t)^{n}}} \int_{\mathbb{R}^{n}} e^{-\frac{\|z-y\|^{2}}{4 t}} x(y) d y, \quad z \in \mathbb{R}^{n} .
$$

Lemma 3.22 guarantees that the dilation symmetry of $\Delta$ implies the dilation symmetry of $\Phi$. Alternatively, this can also be seen making a change of variable in the latter integral.

It is well-known (see Pazy, 1983) that

$$
x\left(t, x_{0}\right)=\Phi(t) x_{0}, \quad t \geq 0
$$

is a unique solution of the linear evolution equation

$$
\dot{x}=A x, \quad t>0,
$$

with the initial condition $x(0)=x_{0} \in \mathbb{B}$. Lemma 3.22 proves the symmetry of these solutions:

$$
x\left(t, \mathbf{d}(s) x_{0}\right)=\mathbf{d}(s) x\left(e^{\mu s} t, x_{0}\right), \quad s \in \mathbb{R}, \quad t \geq 0,
$$

where $x(t, z)$ denotes a solution with the initial condition $x(0)=z$.

The latter identity holds for solutions of non-linear $\mathbf{d}$-homogeneous evolution equations as well.

Theorem 3.23 (Polyakov et al., 2016a; Polyakov, 2020, page 228). Let $\mathbf{d}$ be a group of linear bounded invertible operators on $\mathbb{H}$ and let $A$ and $f$ be $\mathbf{d}$-homogeneous operators of a degree $\mu \in \mathbb{R}$.

If $x:[0, T) \rightarrow \mathbb{H}$ is a mild (strong or classical) solution of the evolution equation (3.25) then the function $x^{s}:\left[0, e^{-\mu s} T\right) \rightarrow \mathbb{B}$ 
defined as

$$
x^{s}(t):=\mathbf{d}(s) x\left(e^{\mu s} t\right), \quad t \in\left[0, e^{-\mu s} T\right)
$$

is also a mild (resp. strong or classical) solution of the evolution equation (3.25).

The latter theorem allows many local properties of homogeneous systems to extend globally. Namely, if solutions of the system (3.25) is well defined for small initial data $x(0)=x_{0} \in \mathbb{H}:\left\|x_{0}\right\|<\varepsilon$ then this system has well-defined solutions for large initial data. Such a property can be utilized for global expansion of regularity of homogeneous evolution systems. Notice that uniques of solutions can also be expanded globally using dilation symmetry.

Corollary 3.24 (Polyakov et al., 2016a). Let $\mathbf{d}$ be a dilation on $\mathbb{H}$. If an evolution system is $\mathbf{d}$-homogeneous and its origin is locally uniformly Lyapunov (asymptotically or finite-time) stable then it is globally uniformly Lyapunov (resp. asymptotically or finite-time) stable.

Since the $\mathbf{d}$-homogeneity of an evolution system is inherited by solutions of this system then it is inherited by a Lyapunov function as well. The following result expands Theorem 3.9 to the abstract evolution equation (3.25).

Theorem 3.25 (Polyakov, 2021b). Let $\mathbf{d}$ be a strongly continuous linear dilation in $\mathbb{H}$ and the system (3.25) be $\mathbf{d}$-homogeneous of degree $\mu \in \mathbb{R}$. Let $m>-\mu$ be an arbitrary number. The origin of (3.25) is uniformly asymptotically stable if and only if there exists a continuous positive definite functional $V: \mathbb{B} \rightarrow \mathbb{R}_{+}$such that

1) $V$ is $\mathbf{d}$-homogeneous of degree $m$ and locally Lipschitz continuous on $\mathbb{B} \backslash\{\mathbf{0}\}$; 
2) $\exists \underline{k}, \bar{k}>0$ satisfying

$$
\underline{k}\|x\|_{\mathbf{d}}^{m} \leq V(x) \leq \bar{k}\|x\|_{\mathbf{d}}^{m}, \quad \forall x \in \mathbb{B} ;
$$

3) for any mild solution $x_{x_{0}}$ of (3.25) with $x(0)=x_{0} \in \mathcal{D}(A) \backslash\{\mathbf{0}\}$ the inequality

$$
\bar{D}^{+} V\left(x_{x_{0}}(t)\right) \stackrel{\text { a.e. }}{\leq}-W\left(x_{x_{0}}(t)\right)
$$

holds as long as $x_{x_{0}}(t) \neq \mathbf{0}$, where $W: \mathbb{B} \backslash\{\mathbf{0}\} \rightarrow \mathbb{R}$ is a positive definite $\mathbf{d}$-homogeneous functional of degree $m+\mu$ such that

$$
\exists \underline{c}>0 \quad: \quad W(x) \geq \underline{c}\|x\|_{\mathbf{d}}^{m+\mu} \quad \forall x \in \mathbb{B} \backslash\{\mathbf{0}\} .
$$

The norm $\|\cdot\|:=\sqrt{\langle\cdot, \cdot\rangle}$ is a usual Lyapunov function for the stability analysis of linear systems (see Lyapunov, 1992, Bacciotti and Rosier, 2001, Curtain and Zwart, 1995, Fridman, 2014) in a Hilbert space $\mathbb{H}$. Indeed, in $\mathbb{R}^{n}$ an inner product can be defined as

$$
\langle x, y\rangle=x^{\top} P y, \quad x, y \in \mathbb{R}^{n}, \quad 0 \prec P=P^{\top} \in \mathbb{R}^{n \times n},
$$

and an existence of a quadratic Lyapunov function $x \rightarrow x^{\top} P x$ is a criterion (necessary and sufficient condition) of stability of any linear system $\dot{x}=A x, A \in \mathbb{R}^{n \times n}$ (see, Lyapunov, 1992). Theorem 3.25 means that a Lyapunov function for a homogeneous system is a kind of homogeneous norm. In particular, the canonical homogeneous induced by the norm $\|\cdot\|:=\sqrt{\langle\cdot, \cdot\rangle}$ is a Lyapunov function for some homogeneous PDEs (Polyakov et al., 2018, Polyakov, 2021b, Polyakov, 2021a).

If is well-known Zubov, 1964, Nakamura et al., 2002 that the homogeneity degree of an asymptotically stable ODE specifies its converge rate.

Corollary 3.26. Let $\mathbf{d}$ be a dilation in $\mathbb{H}$. If the system (3.25) is d-homogeneous of a degree $\nu \in \mathbb{R}$ and its origin is locally uniformly asymptotically stable then 
- for $\nu<0$ it is globally uniformly finite-time stable;

- for $\nu=0$ it is globally uniformly exponentially stable;

- for $\nu>0$ it is globally uniformly nearly fixed-time stable:

$$
\forall r>0, \quad \exists T=T(r)>0:\left\|x\left(t, x_{0}\right)\right\|<r, \forall t \geq T, \forall x_{0} \in \mathbb{H} .
$$

Examples of finite-time stable homogeneous evolution equations in Hilbert spaces are given in Chapter 4. 
Part II

\section{Synthesis of control and estimation algorithms}


As we explained above, the finite-time and fixed-time stability properties have many advantages and are usually accompanied by interesting robustness properties, while many real-world control and estimation applications have a strong need for these convergence characteristics. In this part we will present several constructive solutions for finite-/fixedtime stabilization and observation based on the use of the theory of homogeneity.

In Chapter 4, several control algorithms are presented, which provide accelerated decay rates, and whose design is based on the use of the Implicit Lyapunov function method. In Chapter 5, finite-time and fixedtime observers for linear systems are considered. 


\section{Control Design}

Some motivations for providing in applications the finite/fixed-time convergence rates by a feedback design have been given in Chapter 1. To recall, these properties can be needed in the systems possessing structural reconfigurations or state jumps (e.g., impact dynamics and hybrid systems, when reaching the goal mode is required before the commutation), or they can be deduced as feedback approximations of certain optimization problems (e.g., to avoid undesirable peaking of linear high-gain controls). The system can lose observability in the mode of interest, hence, in such a case the estimation problem has to be solved before approaching the ill-conditioned domain. As it has been discussed in Chapter 2, the resulted kinds of nonlinear dynamics demonstrating faster-than-asymptotic convergence rates also yield remarkable robustness abilities with respect to external disturbances, which can also be demanded in practice.

Homogeneity is a useful tool for finite-time and fixed-time stabilization of a linear or nonlinear system. Indeed, if the closed-loop system is asymptotically stable and homogeneous of a negative degree in $\mathbf{0}$-limit (see Definition 3.11) then it is finite-time stable. If, additionally, it is homogeneous of a positive degree at infinity limit (recall again Defi- 
nition 3.11), then the fixed-time stability is guaranteed as well. The problem of homogeneous stabilization for linear and nonlinear plants is studied in this chapter. However, initially, we extend the scheme of the universal controller design (see Sontag, 1989) to the case of finite-time and fixed-time stabilization.

\subsection{Finite/Fixed-Time stabilization of continuous affine systems}

In this section, we present conditions for accelerated stabilization of continuous autonomous systems (and for brevity only the case of continuous settling-time functions is discussed). To this end, consider the following affine in the control system:

$$
\dot{x}(t)=f_{0}(x(t))+\sum_{i=1}^{m} f_{i}(x(t)) u_{i}(t), \quad x(t) \in \mathbb{R}^{n} \text { and } u(t) \in \mathbb{R}^{m},
$$

where $f_{0}(0)=0, f_{i}$ is continuous for all $0 \leq i \leq m$ and such that (4.1) has uniqueness of solutions in forward time for any $u \in L^{\infty}\left(\mathbb{R}_{+}, \mathbb{R}^{m}\right)$.

Definition 4.1. The system (4.1) is asymptotic/finite/(nearly) fixedtime stabilizable if there exists a feedback control law $u \in C\left(\Gamma, \mathbb{R}^{m}\right)$ such that $u(0)=0$ and the origin of the system (4.1) with this $u=u(x)$ is $\mathrm{AS}$, or FTS/FxTS with a continuous settling-time function, or nearly FxTS, respectively.

Such a feedback law $u(x)$ is called an asymptotic/finite/(nearly) fixed-time stabilizer for the system (4.1).

Following (Sontag, 1989), let us call a positive definite and radially unbounded function $V \in C^{1}\left(\Gamma, \mathbb{R}_{+}\right)$as a control Lyapunov function (CLF) for the system (4.1) if for all $x \in \Gamma \backslash\{0\}$,

$$
\inf _{u \in \mathbb{R}^{m}}\{a(x)+B(x) u\}<0,
$$

where $a(x)=\frac{\partial V(x)}{\partial x} f_{0}(x), B(x)=\left[B_{1}(x) \ldots B_{m}(x)\right]$ with $B_{i}(x)=$ $\frac{\partial V(x)}{\partial x} f_{i}(x)$ for $1 \leq i \leq m$; or equivalently

$$
B(x)=0 \Rightarrow a(x)<0 \quad \forall x \in \Gamma \backslash\{0\} .
$$


Such a CLF satisfies the small control property (SCP) if for each $\epsilon>0$ there exists $\tau>0$ such that: if $\|x\| \leq \tau$, then there exists some $\|u\| \leq \epsilon$ with

$$
a(x)+B(x) u<0 .
$$

The small control property is equivalent to

$$
\limsup _{\|x\| \rightarrow 0} \frac{a(x)}{\|B(x)\|} \leq 0
$$

and this limit may be $-\infty$. It is well-known fact, that (4.1) is asymptotically stabilizable if and only if (4.1) admits a CLF with the SCP (Sontag, 1989). Moreover, the feedback law $u=w(x)$,

$$
w_{i}(x):= \begin{cases}-\frac{a(x)+\sqrt{a(x)^{2}+\|B(x)\|^{4}}}{\|B(x)\|^{2}} B_{i}(x) & \text { if } x \in \Gamma \backslash\{0\} \\ 0 & \text { if } x=0\end{cases}
$$

known as Sontag's universal formula, is a stabilizer for (4.1) (this feedback algorithm is continuous on $\Gamma \backslash\{0\}$, and if $V$ further satisfies the SCP, then $w$ is continuous on $\Gamma$ (Isidori, 1995, Chapter 9)). The following theorems presents an analogous formulation for finite- and (nearly) fixed-time stabilization.

Theorem 4.1 (Moulay and Perruquetti, 2006). The system (4.1) is finite-time stabilizable if there exists a $\operatorname{CLF} V \in C^{1}\left(\Gamma, \mathbb{R}_{+}\right)$that satisfies the SCP and

$$
\sqrt{a(x)^{2}+\|B(x)\|^{4}} \geq c V(x)^{\alpha} \quad \forall x \in \Gamma,
$$

for some $c>0$ and $\alpha \in(0,1)$.

The proof of this theorem utilizes the universal control formula $w(x)$ given above. Another approach, which is based on utilization of the backstepping or the adding power integrator tool, is proposed in the seminal paper (Huang et al., 2005), and its output counterpart in (Qian and $\mathrm{Li}, 2005)$. 
Theorem 4.2 (Lopez-Ramirez et al., 2019). The system (4.1) is fixed-time stabilizable if there exists a CLF $V \in C^{1}\left(\Gamma, \mathbb{R}_{+}\right)$that satisfies the SCP and

$$
\sqrt{a(x)^{2}+\|B(x)\|^{4} \rho(x)^{2}} \geq c_{1} V(x)^{\alpha}+c_{2} V(x)^{\beta} \quad \forall x \in \Gamma,
$$

for some $c_{1}, c_{2}>0, \alpha \in(0,1)$ and $\beta>1$, where $\rho: \Gamma \rightarrow \mathbb{R}$, $\rho \in C(\Gamma \backslash\{0\}, \mathbb{R})$ is such that

$$
\limsup _{x \rightarrow 0}|\rho(x)|\|B(x)\|=0 .
$$

This result is based on a variant of Sontag's universal formula with $u=v(x)$, where for $1 \leq i \leq m$ :

$$
v_{i}(x):=\left\{\begin{array}{ll}
-\frac{a(x)+\sqrt{a(x)^{2}+\|B(x)\|^{4}\left(1+\rho^{2}(x)\right)}}{\|B(x)\|^{2}} & \text { if } x \in \Gamma \backslash\{0\} \\
0 & \text { if } x=0
\end{array} .\right.
$$

Theorem 4.3. The system (4.1) is nearly fixed-time stabilizable if there exists a CLF $V \in C^{1}\left(\Gamma, \mathbb{R}_{+}\right)$that satisfies the SCP and

$$
\sqrt{a(x)^{2}+\|B(x)\|^{4} \rho(x)^{2}} \geq c V(x)^{\beta} \quad \forall x \in \Gamma,
$$

for some $c>0$ and $\beta>1$, where $\rho: \Gamma \rightarrow \mathbb{R}, \rho \in C(\Gamma \backslash\{0\}, \mathbb{R})$ is such that

$$
\limsup _{x \rightarrow 0}|\rho(x)|\|B(x)\|=0 .
$$

Proof. Let us define the feedback law

$$
q_{i}(x):=\left\{\begin{array}{ll}
-\frac{a(x)+\sqrt{a(x)^{2}+b(x)^{2} \tilde{\rho}(x)}}{b(x)} B_{i}(x) & \text { if } x \in \Gamma \backslash\{0\} \\
0 & \text { if } x=0
\end{array},\right.
$$

where $b(x)=\|B(x)\|^{2}$ and $\tilde{\rho}(s):=1+\rho^{2}(x)$ and $1 \leq i \leq m$.

I The derivative of $V$ along the trajectories of (4.1), (4.7) is given 
by

$$
\begin{gathered}
\dot{V}(x)=\left\langle\nabla V(x), f_{0}(x)+\sum_{i=1}^{m} f_{i}(x) q_{i}(x)\right\rangle \\
=-\sqrt{a(x)^{2}+b(x)^{2} \tilde{\rho}(x)}<0 \quad \forall x \in \Gamma \backslash\{0\} .
\end{gathered}
$$

Thus, the control (4.7) is a stabilizer for (4.1) and $V$ is a strict Lyapunov function for the closed-loop system.

II Since $\tilde{\rho}(x) \geq 1$ for all $x \in \Gamma$, using the change of variables $\tilde{B}_{i}(x)=B_{i}(x) \sqrt[4]{\tilde{\rho}(x)}, \tilde{b}(x)=b(x) \sqrt{\tilde{\rho}(x)}$ in (4.7) we obtain

$$
q_{i}(x)= \begin{cases}-\sqrt[4]{1+\rho^{2}(x)} \frac{a(x)+\sqrt{a(x)^{2}+\tilde{b}(x)^{2}}}{\tilde{b}(x)} \tilde{B}_{i}(x) & \text { if } x \in \Gamma \backslash\{0\}, \\ 0 & \text { if } x=0\end{cases}
$$

so that Sontag's universal formula is recovered multiplied by a gain $\sqrt[4]{1+\rho^{2}(x)}$, which is continuous for all $x \in \Gamma \backslash\{0\}$. In addition, this observation also implies that $q(x)=0$ whenever $b(x)=0$. Therefore, for further analysis assume that $b(x) \neq 0$. Since by assumption $V$ satisfies the SCP, $f_{i}: \Gamma \rightarrow \mathbb{R}^{n}$ for $0 \leq i \leq m$ are continuous and $\frac{\partial V}{\partial x}(0)=0$, then for any $\epsilon>0$ there exists $\tau>0$ such that $\sqrt{b(x)} \leq \epsilon$ and

$$
|a(x)| \leq \sqrt{b(x)} \epsilon \leq \epsilon^{2}
$$

for all $0<\|x\| \leq \tau$. Reducing $\tau$ and due to the restrictions imposed on the product $\rho(x) \sqrt{b(x)}$ we also have:

$$
|\rho(x)| \sqrt{b(x)} \leq \epsilon
$$

for all $0<\|x\| \leq \tau$. Recall that

$$
|q(x)|=\frac{\left|a(x)+\sqrt{a(x)^{2}+b(x)^{2} \tilde{\rho}(x)}\right|}{\sqrt{b(x)}},
$$

then if $a(x)>0$ we obtain:

$$
\begin{aligned}
|q(x)| & \leq \frac{2 a(x)+b(x) \sqrt{\tilde{\rho}(x)}}{\sqrt{b(x)}} \\
& \leq 2 \frac{a(x)}{\sqrt{b(x)}}+\sqrt{b(x)}(1+|\rho(x)|) \leq 4 \epsilon .
\end{aligned}
$$


For $a(x) \leq 0$,

$$
a(x)+\sqrt{a(x)^{2}+b(x)^{2} \tilde{\rho}(x)} \leq b(x) \sqrt{\tilde{\rho}(x)}
$$

and

$$
|q(x)| \leq \sqrt{b(x)} \sqrt{\tilde{\rho}(x)} \leq \sqrt{b(x)}(1+|\rho(x)|) \leq 2 \epsilon .
$$

Hence, due to an arbitrary selection of $\epsilon,(4.7)$ is a continuous feedback stabilizer for (4.1).

III Now let us show that the control (4.7) is a fixed-time stabilizer for (4.1). For $\varphi(s):=c s^{\beta}$, we have from (4.9) that

$$
\begin{aligned}
\dot{V}(x) & =-\left(\left(\frac{a(x)}{\varphi(V(x))}\right)^{2}+\left(1+\rho^{2}(x)\right)\left(\frac{b(x)}{\varphi(V(x))}\right)^{2}\right)^{\frac{1}{2}} \varphi(V(x)) \\
& \leq-\left(\frac{a(x)^{2}+\rho(x)^{2} b(x)^{2}}{\varphi(V(x))^{2}}\right)^{\frac{1}{2}} \varphi(V(x)) \\
& \leq-\varphi(V(x)),
\end{aligned}
$$

which implies nearly FxTS for all $x \in \Gamma$.

\subsection{Homogeneous stabilization of linear plants}

\subsubsection{On homogeneity-based finite-time and fixed-time stabiliza- tion}

Let us start with the definition of a kind of stabilizability problem, whose solution will be investigated in this section.

Definition 4.2. A system

$$
\dot{x}=f(x, u), \quad t>0, \quad f: \mathbb{R}^{n} \times \mathbb{R}^{m} \rightarrow \mathbb{R}^{n}
$$

is said to be $\mathbf{d}$-homogeneously stabilizable with a degree $\mu \neq 0$ if there exists a (locally or a globally bounded) feedback law $u: \mathbb{B} \rightarrow \mathbb{X}$ such that the closed-loop system is globally uniformly asymptotically stable and $\mathbf{d}$-homogeneous of the degree $\mu$, where $\mathbf{d}$ is a dilation in $\mathbb{R}^{n}$. 
There are several methods proposing a solution to this stabilization problem (see, for example, Bhat and Bernstein, 2005, Coron and Praly, 1991 and Huang et al., 2005 to mention a few), and below we will present the approach based on implicit Lyapunov function.

\subsubsection{Implicit Lyapunov function-based control design}

Let us consider the linear control system

$$
\dot{x}(t)=A x(t)+B u(x(t)), \quad t>0,
$$

where $x(t) \in \mathbb{R}^{n}$ is the system state, $u: \mathbb{R}^{n} \rightarrow \mathbb{R}^{m}$ is the feedback control, $A \in \mathbb{R}^{n \times n}$ and $B \in \mathbb{R}^{n \times m}$ are system matrices.

In this section we solve the problem of homogeneous stabilization for linear plants in $\mathbb{R}^{n}$ using the canonical homogeneous norm as an implicit Lyapunov function of the closed-loop system. The following result is a corollary of Theorem 7.7 proven below for a Hilbert space $\mathbb{H}$. It uses the canonical homogeneous norm as a Lyapunov function of the closed-loop system and defines a homogeneous stabilizing controller in the implicit form. We also refer the reader to Polyakov et al., 2015a, Polyakov et al., 2016b, Zimenko et al., 2020 for more details about the corresponding analysis in $\mathbb{R}^{n}$.

Theorem 4.4 (Polyakov, 2020). Let $\mathbb{B} \in \mathbb{R}^{n \times m}$ be of a full column rank, i.e.,

$$
\operatorname{rank}(B)=m \leq n .
$$

The linear control system (4.10) is $\mathbf{d}$-homogeneously stabilizable with a degree $\mu \neq 0$ if and only if the pair $\{A, B\}$ is controllable.

Moreover, for $\mu \in\left[-1, k^{-1}\right]$, where $k \leq n: \operatorname{rank}\left(B, A B, \ldots, A^{k-1} B\right)=$ $n$, a homogeneously stabilizing control can always be selected in the form

$$
u(x)=K_{0} x+\|x\|_{\mathbf{d}}^{1+\mu} Y X^{-1} \mathbf{d}\left(-\ln \|x\|_{\mathbf{d}}\right) x
$$

with $K_{0} \in \mathbb{R}^{n \times m}$ such that $A_{0}=A+B K_{0}$ is nilpotent, a linear 
dilation $\mathbf{d}$ generated by $G_{\mathbf{d}} \in \mathbb{R}^{n \times n}$ satisfying

$$
A_{0} G_{\mathbf{d}}=\left(G_{\mathbf{d}}+\mu I\right) A_{0}, \quad G_{\mathbf{d}} B=B
$$

and $X \in \mathbb{R}^{n \times n}, Y \in \mathbb{R}^{m \times n}$ solving the following linear matrix inequalities

$$
\left\{\begin{array}{l}
X A_{0}^{\top}+A_{0} X+Y^{\top} B^{\top}+B Y+\rho\left(X G_{\mathbf{d}}^{\top}+G_{\mathbf{d}} X\right) \preceq 0, \\
X G_{\mathbf{d}}^{\top}+G_{\mathbf{d}} X \succ 0, \quad X \succ 0,
\end{array}\right.
$$

where $\rho>0$ and the canonical homogeneous norm $\|\cdot\|_{\mathbf{d}}$ is induced by the norm $\|x\|_{X^{-1}}=\sqrt{x^{\top} X^{-1} x}$. The canonical homogeneous norm is a Lyapunov function of the closed-loop system (4.10), (4.11) and

$$
\frac{d}{d t}\|x(t)\|_{\mathbf{d}} \leq-\rho\|x(t)\|_{\mathbf{d}}^{1+\mu}
$$

Obviously, the matrix $A_{0}=A+B K_{0}$ is nilpotent if $K_{0}$ solves the zero pole placement problem for the pair $\{A, B\}$ (see Wonham, 1985). There exists several ways to select a matrices $K_{0}$ and $G_{\mathbf{d}}$ (see ,e.g., Misrikhanov and Ryabchenko, 2011, Polyakov et al., 2016b and Zimenko et al., 2020) to fulfill (4.12). If $A$ is nilpotent and $m=1$ then $K_{0}=\mathbf{0 .}$

Remark 4.1. A gain of a linear (already well-tuned) stabilizing control $u_{\text {lin }}(x)=K_{\text {lin }} x$ can be utilized in the design of the homogeneous control (4.11) such that $u(x)=K_{\text {lin }} x$ for $\|x\|_{\mathbf{d}}=1$. A scheme for upgrading a linear feedback to a nonlinear (homogeneous) one is suggested in Polyakov, 2020, page 316. Special tuning rules, which guarantee only an improvement of the control quality without any degradation, are developed in Wang et al., 2020a and demonstrated on a quadrotor control.

The main difficulty of a practical implementation of the control (4.11) is the implicit definition of the canonical homogeneous norm $\|\cdot\|_{\mathbf{d}}$. There are two possible approaches to treat this problem. The first one is based on a development of an appropriate computational scheme for digital implementation of the proposed controller (see Chapter 6). An alternative approach was introduced for the weighted dilation in Zimenko et al., 2018 where $\|\cdot\|_{\mathbf{d}}$ was replaced with an explicitly defined 
homogeneous norm. For linear geometric dilation the suggested scheme is formalized by the following proposition.

Proposition 4.1 (Polyakov, 2018). Let $K_{0} \in \mathbb{R}^{n \times m}$ be such that $A_{0}=A+B K_{0}$ is nilpotent and a dilation $\mathbf{d}$ be generated by an anti-Hurwitz matrix $G_{\mathbf{d}} \in \mathbb{R}^{n \times n}$ satisfying (4.12).

Let for some $0<\delta_{\min } \leq \delta_{\max }$ the system of linear matrix inequalities

$$
\left(\begin{array}{ccc}
A_{0} X+X A_{0}^{\top}+B Y+Y^{\top} B^{\top}-X & \sqrt{\ln \left(\frac{\delta_{\max }}{\delta_{\min }}\right)} B Y & X \mathbf{d}\left(\ln \frac{\delta_{\max }}{\delta_{\min }}\right) \\
\sqrt{\ln \left(\frac{\delta_{\max }}{\delta_{\min }}\right)} Y^{T} B^{T} & -\left(G_{\mathbf{d}}^{-1} X+X G_{\mathbf{d}}^{-\top}\right) & \mathbf{0} \\
\mathbf{d}\left(\ln \frac{\delta_{\max }}{\delta_{\min }}\right) X & \mathbf{0} & -X
\end{array}\right) \prec 0, X \succ 0,
$$

be feasible with respect to $X \in \mathbb{R}^{n \times n}, Y \in \mathbb{R}^{m \times n}$.

If a function $p: \mathbb{R}^{n} \rightarrow \mathbb{R}$ is continuous, positive definite, dhomogeneous of the degree 1 and satisfies the inequalities

$$
0<\delta_{\min } \leq p\left(\frac{z}{\sqrt{z^{\top} X^{-1} z}}\right) \leq \delta_{\max }, \quad \forall z \in \mathbb{R}^{n} \backslash\{\mathbf{0}\},
$$

then the closed-loop system (4.10) with

$$
u(x)=K_{0} x+K \mathbf{d}(-\ln p(x)) x, \quad K=Y X^{-1} \mathbf{d}\left(\ln \delta_{\max }\right)
$$

is globally uniformly finite-time stable and the canonical homogeneous norm $\|\cdot\|_{\mathbf{d}}$ is a Lyapunov function of the closed-loop system.

A possible selection of the homogeneous function $p$ for the controller (4.16) is suggested in the following proposition.

Proposition 4.2 (Polyakov, 2020). Let $\mathbf{d}$ be a strictly monotone continuous dilation in $\mathbb{R}^{n}$ and $\Psi \in C\left(\mathbb{R}^{n}, \mathbb{R}^{n}\right) \cap C^{1}\left(\mathbb{R}^{n} \backslash\{\mathbf{0}\}, \mathbb{R}^{n}\right)$ be 
a homeomorphism on $\mathbb{R}^{n}$ such that

$$
\Psi(\mathbf{d}(s) x)=e^{\rho s} \Psi(x), \quad \forall x \in \mathbb{R}^{n}, s \in \mathbb{R},
$$

where $\rho \in \mathbb{N}$ is a natural number. Then the functional $p: \mathbb{R}^{n} \rightarrow \mathbb{R}$

$$
p(z)=\left(\Psi^{\top}(x) Q \Psi(x)\right)^{\frac{1}{2 \rho}}, \quad 0 \prec Q=Q^{\top} \in \mathbb{R}^{n \times n},
$$

is continuously differentiable outside of the origin, positive definite and $\mathbf{d}$-homogeneous of the degree 1 , i.e., $p(\mathbf{d}(s) x)=e^{s} p(x)$ for all $s \in \mathbb{R}, \forall x \in \mathbb{R}^{n}$.

Moreover, if $\Delta=\left\{z_{i}\right\}_{i=1}^{N}$ is a sufficiently dense grid on the unit sphere $S=\left\{x \in \mathbb{R}^{n}:\|x\|=1\right\}$ such that

$$
\inf _{i}\left\|z-z_{i}\right\|_{P} \leq \varepsilon<1, \quad \forall z \in S
$$

and

$$
\sup _{i}\left|p\left(z_{i}\right)-1\right| \leq \delta_{0}
$$

then

$$
0<1-\delta \leq p(z) \leq 1+\delta, \quad \forall z \in S
$$

where

$$
\delta=\delta_{0}+\left.\varepsilon \sup _{y, z \in S, 0 \leq \theta<\varepsilon} \frac{\partial p(x)}{\partial x}\right|_{x=y+\theta z} z
$$

for $\|x\|_{P}=\sqrt{x^{\top} P x}$ with $P$ satisfying (3.10).

Since $|p(x)-1|=\left|p(x)-\|x\|_{\mathbf{d}}\right|$ if $\|x\|_{P}=1$ then for $\delta=0$ we have $p(x)=\|x\|_{\mathbf{d}}$ due to homogeneity, i.e., the latter proposition suggests a numerical scheme for an approximation of the canonical $\mathbf{d}$-homogeneous norm.

\section{Algorithm 4.1.}

Initialization. Select a small $\varepsilon \in(0,1)$, a natural $\rho \in \mathbb{N}$ and $\Psi$ satisfying conditions of Proposition 4.2. Find $P$ satisfying (3.10).

Step 1. Generate randomly (uniformly distributed) non-zero vectors $\tilde{z}_{i} \in[-1,1]^{n}, i=1,2, \ldots, N$ and project them to the unit sphere $z_{i}:=\frac{\tilde{z}_{i}}{\left\|\tilde{z}_{i}\right\|_{P}}$, where $\left\|\tilde{z}_{i}\right\|_{P}=\sqrt{z_{i}^{\top} P z_{i}}$. 
Step 2. If the inequality (4.18) does not hold for the given $\varepsilon>0$ then repeat Step 1 for $N=2 N$.

Step 3. Solve the following semi-definite programming (SDP) problem (see ,e.g., Boyd et al., 1994 for more details about SDP)

$$
\operatorname{minimize} \sum_{i=1}^{N}\left(\Psi^{\top}\left(z_{i}\right) Q \Psi\left(z_{i}\right)\right)^{2}-2\left(\Psi^{\top}\left(z_{i}\right) Q \Psi\left(z_{i}\right)\right)
$$

subject to $0 \prec Q=Q^{\top} \in \mathbb{R}^{n \times n}$.

It is easy to see that

$$
\begin{gathered}
\left(\Psi^{\top}\left(z_{i}\right) Q \Psi\left(z_{i}\right)\right)^{2}-2\left(\Psi^{\top}\left(z_{i}\right) Q \Psi\left(z_{i}\right)\right)= \\
p^{4 \rho}\left(z_{i}\right)-2 p^{2 \rho}\left(z_{i}\right)=\left(p_{2 \rho}\left(z_{i}\right)-1\right)^{2}-1,
\end{gathered}
$$

i.e., the above minimization problem is aimed at minimization of the difference $\left|p\left(z_{i}\right)-1\right|$ for all $z_{i} \in S$.

Example 4.1. To illustrate the above theoretical results we consider the system

$$
\dot{x}=\left(\begin{array}{ll}
0 & 1 \\
0 & 0
\end{array}\right) x+\left(\begin{array}{l}
0 \\
1
\end{array}\right) u(x), \quad u: \mathbb{R}^{2} \rightarrow \mathbb{R}
$$

and the weighted dilation

$$
\mathbf{d}(s)=e^{s G_{\mathbf{d}}}, \quad G_{\mathbf{d}}=\left(\begin{array}{ll}
5 & 0 \\
0 & 4
\end{array}\right), \quad s \in \mathbb{R} .
$$

Selecting $\delta_{\min }=0.99, \delta_{\max }=1.01$ we solve the linear matrix inequality (4.14) and obtain:

$$
P=X^{-1}=\left(\begin{array}{ll}
0.1157 & 0.0194 \\
0.0194 & 0.1186
\end{array}\right)
$$

and

$$
K=Y X^{-1} \mathbf{d}\left(\ln \delta_{\max }\right)=-\left(\begin{array}{ll}
-1.1096 & -0.6944
\end{array}\right) .
$$

Next, we select $\rho=5, \Psi(x)=\left(\begin{array}{c}x_{1} \\ \left|x_{2}\right|^{5 / 4} \operatorname{sign}\left(x_{2}\right)\end{array}\right)$ and select

$$
Q=\left(\begin{array}{ll}
0.1140 & 0.0164 \\
0.0164 & 0.0689
\end{array}\right) \text {. }
$$


One can be checked numerically that $\left|p(x)-\|x\|_{\mathbf{d}}\right| \leq 0.01$ for $x \in S$, where $p$ is given by (4.17).

Theorem 4.4 has the following straightforward corollary.

Corollary 4.5. Let $K_{0} \in \mathbb{R}^{m \times n}$ be such that $A+B K_{0}$ is nilpotent. Let anti-Hurwitz matrices $G_{\mathbf{d}_{1}} \in \mathbb{R}^{n \times n}$ and $G_{\mathbf{d}_{2}} \in \mathbb{R}^{n \times n}$ satisfy the following identities:

$$
A_{0} G_{\mathbf{d}_{i}}=\left(G_{\mathbf{d}_{i}}+\mu_{i} I_{n}\right) A_{0}, \quad G_{\mathbf{d}_{i}} B=B, \quad i=1,2
$$

for some $\mu_{1} \in[-1,0)$ and some $\mu_{2}>0$. If $X \in \mathbb{R}^{n \times n}$ and $Y \in \mathbb{R}^{m \times n}$ satisfy the following system of linear matrix inequalities

$$
\left\{\begin{array}{l}
X A_{0}^{\top}+A_{0} X+Y^{\top} B^{\top}+B Y+\rho_{i}\left(X G_{\mathbf{d}}^{\top}+G_{\mathbf{d}} X\right) \preceq \mathbf{0}, \\
X G_{\mathbf{d}_{i}}^{\top}+G_{\mathbf{d}_{i}} X \succ 0, \quad X \succ 0, \quad i=1,2
\end{array}\right.
$$

for some $\rho_{1}>0$ and $\rho_{2}>0$, then the control

$$
u(x)=K_{0} x+Y X^{-1}\left\{\begin{array}{lll}
\|x\|_{\mathbf{d}_{1}}^{1+\mu_{1}} \mathbf{d}_{1}\left(-\ln \|x\|_{\mathbf{d}_{1}}\right) x & \text { if } & \|x\|_{X^{-1}} \leq 1, \\
\|x\|_{\mathbf{d}_{2}}^{1+\mu_{2}} \mathbf{d}_{2}\left(-\ln \|x\|_{\mathbf{d}_{2}}\right) x & \text { if } & \|x\|_{X^{-1}}>1
\end{array}\right.
$$

steers any trajectory of the system (4.10) to zero in a fixed time

$$
T\left(x_{0}\right) \leq \frac{1}{-\mu_{1} \rho_{1}}+\frac{1}{\mu_{2} \rho_{2}}
$$

independently of the initial condition, where $T: \mathbb{R}^{n} \rightarrow \mathbb{R}$ is a settling-time function and the canonical homogeneous norms $\|\cdot\|_{\mathbf{d}_{1}}$ and $\|\cdot\|_{\mathbf{d}_{2}}$ are induced by the norm $\|x\|_{X^{-1}}=\sqrt{x^{\top} X^{-1} x}$.

The following corollary treats the case of finite-time stabilization under exogenous perturbations. 
Corollary 4.6 (Polyakov, 2020). Let all conditions of Corollary 4.4 hold and $f \in C\left(\mathbb{R} \times \mathbb{R}^{n}, \mathbb{R}^{n}\right)$ satisfy the following inequality

$$
\frac{\left\|\mathbf{d}\left(-\ln \|x\|_{\mathbf{d}}\right) f(t, x)\right\|_{P}}{x^{\top} \mathbf{d}^{\top}\left(-\ln \|x\|_{\mathbf{d}}\right) P G_{\mathbf{d}} \mathbf{d}\left(-\ln \|x\|_{\mathbf{d}}\right) x} \leq \kappa\|x\|_{\mathbf{d}}^{\mu}, \quad \forall x \in \mathbb{R}^{n} \backslash\{\mathbf{0}\}, \forall t \geq 0
$$

for some $\kappa>0$, where the canonical homogeneous norm $\|\cdot\|_{\mathbf{d}}$ is induced by $\|x\|_{P}=\sqrt{x^{\top} P x}, P=X^{-1}$. If, additionally,

$$
\left(A_{0}+B K\right)^{\top} P+P\left(A_{0}+B K\right)+(\rho+\kappa)\left(G_{\mathbf{d}}^{\top} P+P G_{\mathbf{d}}\right) \preceq 0, \quad \rho>0,
$$

then Corollary 4.4 remains true for the system

$$
\dot{x}=A x+B u(x)+f(t, x), \quad t>0 .
$$

Notice that, in particular, if $\mu=-1$, and $f(t, x)=B \Gamma(t, x)$ for some $\Gamma: \mathbb{R} \times \mathbb{R}^{n} \rightarrow \mathbb{R}^{m}$ then $\mathbf{d}(s) B=e^{s} B$ and the inequality (4.23) becomes

$$
\frac{\|B \Gamma(t, x)\|_{P}}{x^{\top} \mathbf{d}^{\top}\left(-\ln \|x\|_{\mathbf{d}}\right) P G_{\mathbf{d}} \mathbf{d}\left(-\ln \|x\|_{\mathbf{d}}\right) x} \leq \kappa, \quad \forall x \in \mathbb{R}^{n} \backslash\{\mathbf{0}\}, \forall t \geq 0 .
$$

Since $\mathbf{d}\left(-\ln \|x\|_{\mathbf{d}}\right) x \in S$ and $G_{\mathbf{d}}^{\top} P+P G_{\mathbf{d}} \succ 0$ then

$$
c:=\inf x^{\top} \mathbf{d}^{\top}\left(-\ln \|x\|_{\mathbf{d}}\right) P G_{\mathbf{d}} \mathbf{d}\left(-\ln \|x\|_{\mathbf{d}}\right) x>0,
$$

i.e., for $\mu=-1$ and $G_{\mathbf{d}} B=B$ the homogeneous control (4.11) rejects uniformly bounded perturbations $B \Gamma$ of a magnitude $\kappa c>0$ provided that the LMI (4.24) holds. For $\mu \geq-1$ the controller (4.11) rejects some additive perturbation vanishing at $x=0$ (see Polyakov et al., 2016b and Zimenko et al., 2020 for more details).

The numerical simulation results for the implicit homogeneous finitetime controller are presented in Chapter 6 .

\subsection{Homogeneous stabilization of nonlinear plants}

In the previous section, the Control Lyapunov function-based approach for affine systems was presented for accelerated stabilization. In this section we will briefly describe other useful tools solving the same problem based on homogeneity. 


\subsubsection{Dynamic feedback design for homogeneous stabilization of nonlinear plants}

In this subsection we consider the problem of homogeneous stabilizability of a system

$$
\dot{\sigma}(t)=g(\sigma(t), u(t)), \quad g: \mathbb{R}^{n} \times \mathbb{R}^{m} \rightarrow \mathbb{R}^{n}
$$

by means of a dynamical feedback

$$
\dot{u}(t)=k(\sigma(t), u(t)), \quad k: \mathbb{R}^{n} \times \mathbb{R}^{m} \rightarrow \mathbb{R}^{m} .
$$

In this case, the system can be treated as affine in control and based on the scheme of universal stabilizing control design proposed in (Sontag, 1989) we derive the following corollary of Theorem 3.10.

Theorem 4.7 (Polyakov, 2018). Let $\mathbf{d}_{x}$ and $\mathbf{d}_{u}$ be linear dilations in $\mathbb{R}^{n}$ and $\mathbb{R}^{m}$, respectively,

$$
\mathbf{d}(s):=\left(\begin{array}{cc}
\mathbf{d}_{x}(s) & \mathbf{0} \\
\mathbf{0} & \mathbf{d}_{u}(s)
\end{array}\right)
$$

and the vector field $\tilde{f}: \mathbb{R}^{n} \times \mathbb{R}^{m} \rightarrow \mathbb{R}^{n} \times \mathbb{R}^{m}, \tilde{f}=\left(\begin{array}{c}g \\ \mathbf{0}\end{array}\right)$ be $\mathbf{d}$ homogeneous of a degree $\mu \in \mathbb{R}$. The origin of the system (4.26) is globally uniformly $\mathbf{d}$-homogeneously stabilizable with the degree $\mu$ if and only if there exist a number $\gamma \geq 0$, a symmetric matrix $P \in \mathbb{R}^{(n+m) \times(n+m)}$ satisfying (3.10) and a mapping

$$
\Xi \in C^{\infty}\left(\mathbb{R}^{n+m} \backslash\{\mathbf{0}\}, \mathbb{R}^{(n+m) \times(n+m)}\right)
$$

such that

$$
\operatorname{det}(\Xi(z)) \neq 0, \frac{\partial \Xi(z)}{\partial z_{i}} z=0, \Xi\left(e^{s} z\right)=\Xi(z)
$$

for all $z=\left(z_{1}, . ., z_{n+m}\right) \in \mathbb{R}^{n+m} \backslash\{\mathbf{0}\}, s \in \mathbb{R}, i=1, \ldots, n+m$ and

$$
a(z)<\gamma \sqrt{b^{\top}(z) b(z)} \quad \text { for } \quad z \in S,
$$


where

$$
\begin{gathered}
a(z)=z^{\top} W(z) \tilde{f}(z), \quad b^{\top}(z)=z^{\top} W(z)\left(\begin{array}{c}
\mathbf{0} \\
I_{m}
\end{array}\right), \\
W(z)=\Xi^{\top}(z) P \Xi(z)\left(\frac{\left(I_{n}-G_{\mathbf{d}}\right) z^{\top} z P}{z^{\top} P G_{\mathbf{d}} z}+I_{n}\right)
\end{gathered}
$$

and $S$ is the unit sphere in $\mathbb{R}^{n+m}$ with $\|z\|_{P}=\sqrt{z^{\top} P z}$. Moreover, the corresponding stabilizing homogeneous feedback law can be designed as follows

$k(\xi)=\|\xi\|_{\mathbf{d}}^{\mu} \mathbf{d}_{u}\left(\ln \|\xi\|_{\mathbf{d}}\right) k_{0}\left(\mathbf{d}\left(-\ln \|\xi\|_{\mathbf{d}}\right) \xi\right) \quad$ with $\quad \xi=\left(\begin{array}{c}\sigma \\ u\end{array}\right)$

where

$$
k_{0}(\cdot)=\left\{\begin{array}{cl}
-\frac{a(\cdot)+\sqrt{a^{2}(\cdot)+\left(b^{\top}(\cdot) b(\cdot)\right)^{2}}}{b^{\top}(\cdot) b(\cdot)} b(\cdot) & \text { if } b(\cdot) \neq \mathbf{0} \\
\mathbf{0} & \text { if } b(\cdot)=\mathbf{0}
\end{array}\right.
$$

and $\|\cdot\|_{\mathbf{d}}$ is the canonical homogeneous norm induced by $\|\cdot\|_{P}$.

Notice that the matrix valued function $\Xi$ is constant along any ray $\lambda \rightarrow \lambda z$. It can be assumed constant in many practical cases.

Example 4.2. Let us consider the stabilization problem for the system

$$
\dot{\sigma}_{1}=\left|\sigma_{1}\right|^{\frac{1}{3}} u+\sigma_{2}, \quad \dot{\sigma}_{2}=u,
$$

where $\sigma_{1}, \sigma_{2}, u \in \mathbb{R}$. The vector field $\tilde{f}: \mathbb{R}^{3} \rightarrow \mathbb{R}^{3}$ defined by

$$
\tilde{f}(\xi)=\left(\begin{array}{c}
\left|\xi_{1}\right|^{\frac{1}{3}} \xi_{3}+\xi_{2} \\
\xi_{3} \\
0
\end{array}\right),
$$

$\xi=\left(\xi_{1}, \xi_{2}, \xi_{3}\right)^{\top}=\left(\sigma_{1}, \sigma_{2}, u\right)^{\top}$ is $\mathbf{d}$-homogeneous of degree -1 with respect to the dilation $\mathbf{d}$ in $\mathbb{R}^{3}$ generated by $G_{\mathbf{d}}=\operatorname{diag}\{3,2,1\}$. Hence, the stabilizability condition (4.28) with $\Xi=$ const becomes

$$
z^{\top} P A(z) z<\gamma \sqrt{z^{\top} P z} \sqrt{z^{\top} P e_{3} e_{3}^{\top} P z},
$$

$P>0, P G_{\mathbf{d}}+G_{\mathbf{d}}^{\top} P>0$, where $z=\left(z_{1}, z_{2}, z_{3}\right)^{\top} \in \mathbb{R}^{3}$ and

$$
A(z)=\left(\begin{array}{ccc}
0 & 1 & \left(\frac{\left|z_{1}\right|}{\sqrt{z^{\top} P z}}\right)^{\frac{1}{3}} \\
0 & 0 & 1 \\
0 & 0 & 0
\end{array}\right) .
$$


Let $X \in \mathbb{R}^{3 \times 3}$ and $\gamma_{0}>0$ satisfy the linear matrix inequalities

$$
X A_{i}+A_{i}^{\top} X-2 \gamma e_{3} e_{3}^{\top}<0, \quad X G_{\mathbf{d}}+G_{\mathbf{d}} X>0, \quad X>0
$$

$A_{i}=\left(\begin{array}{lll}0 & 1 & i \\ 0 & 0 & 1 \\ 0 & 0 & 0\end{array}\right), i=0,1, e_{3}=(0,0,1)^{\top} \in \mathbb{R}^{3}$. Since $A(z)=\alpha(z) A_{1}+$ $(1-\alpha(z)) A_{0}$, where $\alpha(z)=\left(\frac{\left|z_{1}\right|}{\sqrt{z^{\top} P z}}\right)^{\frac{1}{3}}$ and $\alpha(z) \in[0,1]$ provided that $p_{11}=1$, then for $P=\tilde{P} /\left(\tilde{p}_{11}\right), \tilde{P}=X^{-1}$ the stabilizability condition (4.28) holds. So, the considered system can be stabilized in a finite time (due to $\operatorname{deg}_{\mathbf{d}}(\tilde{f})=-1$ ) to zero by means of the $\mathbf{d}$-homogeneous dynamical feedback

$$
\dot{u}=k_{0}\left(\mathbf{d}\left(-\ln \|\xi\|_{\mathbf{d}}\right) \xi\right),
$$

where $k_{0}$ is given by (4.30) with $a(z)=z^{\top} P \tilde{f}(z)$ and $b(z)=p_{13} z_{1}+p_{23} z_{2}+$ $p_{33} z_{3}$. For example, the appropriate matrix $P$ obtained using the above LMIs is

$$
P=\left(\begin{array}{lll}
1.0000 & 0.8930 & 0.8846 \\
0.8930 & 1.8679 & 1.3659 \\
0.8846 & 1.3659 & 1.7523
\end{array}\right)
$$

\subsubsection{Finite-time stabilization in sliding mode control theory}

The sliding mode control theory uses the finite-time stabilization properties of relay feedbacks (Utkin, 1992), (Shtessel et al., 2014). For example, let us consider a nonlinear affine system

$$
\begin{gathered}
\dot{x}(t)=a(x(t))+b(x(t)) u(t), t \geq 0, \\
a: \mathbb{R}^{n} \rightarrow \mathbb{R}^{n}, b: \mathbb{R}^{n} \rightarrow \mathbb{R}^{n \times m}, x(t) \in \mathbb{R}^{n}, u(t) \in \mathbb{R}^{m} .
\end{gathered}
$$

The aim of the sliding mode controller it to steer all trajectories of the system to an $m$-dimensional surface $s(x)=0), s: \mathbb{R}^{n} \rightarrow \mathbb{R}^{m}$ in a finite time. If $\frac{\partial s}{\partial x} b(x)$ is invertible for all $x \in \mathbb{R}^{n}$ then the required controller can be selected as follows (Utkin, 1992)

$$
u=-M(x)\left[\frac{\partial s}{\partial x} b(x)\right]^{-1} \operatorname{sign}(s),
$$

where the scalar-valued function $M: \mathbb{R}^{n} \rightarrow \mathbb{R}_{+}$can be defined as follows $M(x)=\left\|\frac{\partial s}{\partial x} a(x)\right\|+\delta$ with an arbitrary $\delta>0$. Indeed, the closed-loop 
dynamics of $s$ is given by

$$
\dot{s}=\frac{\partial s}{\partial x} a(x)+\frac{\partial s}{\partial x} b(x) u=\frac{\partial s}{\partial x} a(x)-\left(\left\|\frac{\partial s}{\partial x} a(x)\right\|+\delta\right) \operatorname{sign}(s(x)) .
$$

The state $s=0$ of the latter equation is, obviously, finite time stable with the settling time estimate $T(s(x(0))) \leq \delta\|s(x(0))\|$. Solutions of closed-loop (discontinuous) system are defined using some regularization approach. The most famous one is Filippov's theory (see Filippov, 1988 for more details). 


\section{5}

\section{State Estimation}

Usually the state estimation is needed for the output control design or for construction of a monitoring system.

Assume in this section that the controlled plant is described by a linear differential equation:

$$
\left\{\begin{array}{l}
\dot{x}=A x+B u, \\
y=C x,
\end{array}\right.
$$

where $x \in \mathbb{R}^{n}$ is the state variable, $y \in \mathbb{R}^{k}$ is the measured output, $u: \mathbb{R} \rightarrow \mathbb{R}^{m}$ is an input, $A \in \mathbb{R}^{n \times n}$ is the system matrix, $B \in \mathbb{R}^{n \times m}$ is the matrix of input gains and the matrix $C \in \mathbb{R}^{k \times n}$ models the measurements of the state variables. In such a case the output feedback synthesis benefits the separation principle (Khalil, 2002): the observer $\dot{z}=A z+B u+L(y-C z)$, where $z \in \mathbb{R}^{n}$ is the estimate of $x$ with the observer gain $L \in \mathbb{R}^{n \times k}$, and the feedback control $u=K x$ with the control gain $K \in \mathbb{R}^{m \times n}$, can be designed independently. In other words, by a choice of the gains $L$ and $K$ it is sufficient to ensure that the matrices $A-L C$ and $A+B K$ are Hurwitz, then the system (5.1) closed by the observer-based feedback $u=K z$ is globally asymptotically stable. However, the nominal quality of the control can be recovered only when the estimation error $e=x-z$ between the estimates and the 
real value of the state becomes sufficiently small. Obviously, the control gain $K$ is not selected just to provide convergence of the state $x$, but usually it serves to optimize a functional on the system trajectories, then minimization of the discrepancy $e$ as fast as possible becomes important, especially if the initial deviation in $e$ may be significantly large. As it has been explained in Chapter 1 by considering the stabilization problem (it is the same issue with the observers), augmentation of the gain $L$ may accelerate the time of convergence, but at the price of the peaking, which is a huge and unwanted overshoot in the transients of $e$. Consequently, other solutions have to be found, and design of a finite-time or fixed-time converging observer is a possible direction of research.

The situation becomes even more complicated if the controlled plant's model is nonlinear, when the separation principle does not work (Khalil, 2002). In such a case, the observer may ensure asymptotic estimation of the state, the controller guarantees a respective regulation of the plant by a state feedback, but substitution of the state estimation in the control leads to serious degradation of the performances, or even finite-time escape of the trajectories. Hence, the common solution is a simultaneous design of the controller and the observer. Another way of avoiding such an instability can be a separate their design (which is more simple), but with the observer converging uniformly and faster than extinction of the plant trajectories with the observer-based feedback. Therefore, finite/fixed-time estimation may recover a kind of separation principle in the nonlinear context (an example is provided in Chapter 1). In general, having a fixed-time observer with a globally bounded convergence time also simplifies forthcoming analysis of the system (monitor design, fault detection), since the observer dynamics after convergence can be just neglected.

Finally, if we would like to stabilize a system in finite/fixed-time using an observer-based feedback, then as usual the observer has to converge faster than the controller. Indeed, if the control is synthesized to ensure a finite-time convergence to the origin, while the state estimation error converges asymptotically to zero, the total rate of decay for the observer-based stabilization control will follow the weaker element, it will be also asymptotic. 


\subsection{Problem statement}

Similarly to the homogeneous stabilization problem we can consider the problem of a homogeneous observation (state estimation) for the system (5.1).

Definition 5.1. The system (5.1) is said to be $\mathbf{d}$-homogeneously observable of a degree $\nu \in \mathbb{R}$ if there exists a dynamic observer of the form

$$
\dot{z}=A z+B u+g(C z-y), \quad g: \mathbb{R}^{k} \rightarrow \mathbb{R}^{n}
$$

such that the error equation

$$
\dot{e}=A e+g(C e), \quad e=z-x
$$

is globally uniformly asymptotically stable and $\mathbf{d}$-homogeneous of the degree $\nu \in \mathbb{R}$.

Notice that the uniform asymptotic stability and the $\mathbf{d}$-homogeneity of the error equation (5.3) yield its finite-time stability if $\nu<0$ and the nearly fixed-time stability if $\nu>0$ (see Theorem 3.26).

Our goals are

- to find necessary and sufficient conditions of the $\mathbf{d}$-homogeneous observability of (5.1);

- to design a d-homogeneous observer of the form 5.2 for $\nu \in \mathbb{R}$.

Since, in this chapter, $e$ denotes the estimation error then to avoid confusing notations the exponential function $\left(s \rightarrow e^{s}\right.$, where $e$ is the Euler number) is denoted $s \rightarrow \exp (s)$.

\subsection{Observer design for a homogeneous linear plant}

In Section 4.2.2 a homogeneous controller for a linear plant has been designed by means of a nonlinear (norm-dependent) dilation of a linear 
feedback. The paper Lopez-Ramirez et al., 2018 follows the same idea in order to design a homogeneous observer using weighted homogeneity. The constructions presented in the mentioned paper can be simplified using the linear geometric homogeneity.

Theorem 5.1 (Polyakov, 2020). Let $C \in \mathbb{R}^{k}$ be a full row rank matrix and $G_{0} \in \mathbb{R}^{n \times n}$ satisfy the identities

$$
A G_{0}=\left(G_{0}+I_{n}\right) A, \quad C G_{0}=\mathbf{0} .
$$

Let $\nu \in \mathbb{R}$ be such that real parts of the eigenvalues of the matrix $I_{n}+\nu\left(I_{n}+G_{0}\right)$ are non-negative.

Let $P \in \mathbb{R}^{n \times n}, \rho>1, \gamma>0$ and $\nu \in \mathbb{R}$ satisfy the system of matrix inequalities

$$
\begin{gathered}
P A+A^{\top} P+\rho P-\gamma C^{\top} C \prec 0, \quad\left(\begin{array}{cc}
P & \gamma C^{\top} \\
\gamma C & I_{k}
\end{array}\right) \succ 0, \\
P\left(I_{n}+\nu G_{0}\right)+\left(I_{n}+\nu G_{0}\right)^{\top} P \succ 0, \quad(5) \\
P^{-1} \succ \Xi(\lambda) P^{-1} C^{\top} C P^{-1} \Xi^{\top}(\lambda), \quad \forall \lambda \in[0,1],
\end{gathered}
$$

where the matrix-valued function $\lambda \rightarrow \Xi(\lambda)$ is given by

$$
\Xi(\lambda)=\lambda\left(\exp \left(\ln \lambda^{\nu}\left(G_{0}+I_{n}\right)\right)-I_{n}\right) .
$$

Then the dynamic observer (5.2) with the locally bounded function $g \in C\left(\mathbb{R}^{k} \backslash\{\mathbf{0}\}, \mathbb{R}^{n}\right)$ given by

$$
g(\sigma)=-\frac{\gamma}{2}\|\gamma \sigma\|_{\mathbb{R}_{k}}^{\nu} \exp \left(\ln \|\gamma \sigma\|_{\mathbb{R}^{k}}^{\nu} G_{0}\right) P^{-1} C^{\top} \sigma, \quad \sigma \in \mathbb{R}^{k}
$$

has a globally uniformly asymptotically stable $\mathbf{d}$-homogeneous error equation (5.3) of the degree $\nu \in \mathbb{R}$ and

$$
\frac{d}{d t}\|e(t)\|_{\mathbf{d}} \leq-\frac{(\rho-1)\|e(t)\|_{\mathbf{d}}^{1+\nu}}{\lambda_{\max }\left(P^{-\frac{1}{2}} G_{\mathbf{d}}^{\top} P^{\frac{1}{2}}+P^{\frac{1}{2}} G_{\mathbf{d}} P^{-\frac{1}{2}}\right)},
$$

where the linear dilation $\mathbf{d}$ is generated by $G_{\mathbf{d}}=I_{n}+\nu G_{0}$ and $\|\cdot\|_{\mathbb{R}^{k}}$ is the standard Euclidean norm in $\mathbb{R}^{k}$. Moreover, $g$ is continuous at zero provided that the matrix $I_{n}+\nu\left(I_{n}+G_{0}\right)$ is anti-Hurwitz. 
The next proposition studies the feasibility of the matrix inequalities (5.5), (5.6).

Proposition 5.1. If the pair $\{A, C\}$ is observable, then the system of the matrix inequalities $(5.5),(5.6)$ is always feasible for a sufficiently small $|\nu|$.

Notice that for $\nu=0$ we have $\Xi \equiv \mathbf{0}, g(\sigma)=-\frac{\gamma}{2} P^{-1} C^{\top} \sigma$ and the observer (5.2) becomes a linear Luenberger observer. For $\nu<0$ we derive a finite-time observer. If $\nu>0$ the homogeneous observer guarantees a nearly fixed-time stability of the error equation. Another homogeneity-based solution is presented in (Andrieu et al., 2008).

\subsection{On selection of observer's parameters}

In order to apply Theorem 5.1 we need to solve the parametrized system of matrix inequalities (5.5), (5.6) with respect to variables $P$ and $\gamma$ for a given $\nu \in \mathbb{R}$. By fixing $\lambda \in[0,1]$, the system (5.5), (5.6) becomes a system of LMIs, which can be solved using any appropriate mathematical software (e.g., MATLAB ${ }^{T M}$ ). However, the mentioned LMIs must be fulfilled for all $\lambda \in[0,1]$. This can be checked on a proper grid on the interval $[0,1]$ due to the smoothness of the matrix-valued function $\Xi$. The next corollary presents sufficient feasibility conditions for the parametrized matrix inequality (5.5).

Proposition 5.2. The parametric inequality (5.6) holds if

$$
\frac{1}{|\nu|} \Xi^{\top}\left(q_{i}\right) P \Xi\left(q_{i}\right) \prec\left(1-\frac{1}{2}\left(q_{i}^{2}-q_{i-1}^{2}\right)\right) P, \quad i=1, \ldots, N,
$$

$2 P+\nu P\left(G_{0}^{\top}+I_{n}\right)+\nu\left(G_{0}+I_{n}\right) P \succeq|\nu|\left(G_{0}^{\top}+I_{n}\right) P\left(G_{0}+I_{n}\right), P \succeq|\nu| C^{\top} C$

where $0=q_{0}<q_{1}<\ldots<q_{N}=1$. 
Notice that the system of inequalities (5.5), (5.9), (5.10) is linear with respect to $P$ and $\gamma$. Based on Proposition 5.2 the following simple algorithm for a selection of the observer's parameters can be proposed.

Algorithm 5.1. Initialization: $N=1, q_{0}=0, q_{N}=1, \Sigma=\left\{q_{0}, q_{N}\right\}$.

Loop: While the system of LMIs (5.5), (5.9), (5.10) is not feasible, do $\Sigma \leftarrow \Sigma \cup\left\{\frac{q_{i-1}+q_{i}}{2}\right\}_{i=1}^{N}$ and $N \leftarrow 2 N$.

Since $\sup _{\lambda \in[0,1]} \sqrt{|\nu|^{-1}}\|\Xi(\lambda)\| \rightarrow 0$ as $\nu \rightarrow 0$ (see the proof of Proposition 5.1 for more details) then the presented algorithm always finds a solution of the system (5.5), (5.6) provided that $|\nu|$ is sufficiently small and the pair $\{A, C\}$ is observable.

\subsection{Necessary and sufficient condition of homogeneous observabil- ity}

According to the Kalman duality principle a state estimation problem for a linear system corresponds to a stabilization problem for an adjoint system. In the view of the results of Chapter 4, the necessary and sufficient condition of the $\mathbf{d}$-homogeneous observability is expected to be the classical observability condition of linear plants.

Theorem 5.2 (Polyakov, 2020). The system (5.1) is d-homogeneously observable of a degree $\nu \neq 0$ if and only if the pair $\{A, C\}$ is observable, i.e.,

$$
\operatorname{rank}\left[\begin{array}{c}
C \\
C A \\
C A^{2} \\
\cdots \\
C A^{n-1}
\end{array}\right]=n .
$$

Moreover, a homogeneous observer can always be designed in 
the form (5.2) with

$$
g(\sigma)=L_{0} \sigma-\frac{\gamma}{2}\|\sigma\|_{\mathbb{R}_{k}}^{\nu} \exp \left(\ln \|\sigma\|_{\mathbb{R}^{k}}^{\nu} G_{0}\right) P^{-1} C^{\top} \sigma, \quad \sigma \in \mathbb{R}^{k},
$$

where $L_{0} \in \mathbb{R}^{n \times k}$ is such that $A_{0}=A+L_{0} C$ is nilpotent, $G_{0} \in \mathbb{R}^{n \times n}$ fulfills

$$
A_{0} G_{0}=\left(G_{0}+I_{n}\right) A_{0}, \quad C G_{0}=\mathbf{0},
$$

$P \in \mathbb{R}^{n \times n}$ and $\gamma>0$ satisfy the matrix inequalities (5.5), (5.6), and $\nu \in \mathbb{R}$ is such that real parts of the eigenvalues of the matrix $I_{n}+\nu\left(I_{n}+G_{0}\right)$ are non-negative.

\subsection{Robustness Analysis}

Let us consider a linear control system with unknown perturbations and measurement noises:

$$
\left\{\begin{array}{l}
\dot{x}=A x+B u+q_{x}, \\
y=C x+q_{y},
\end{array}\right.
$$

where, as before, $x$ is the state variable, $y$ is the measured output, $u: \mathbb{R} \rightarrow \mathbb{R}^{m}$ is an input, $A \in \mathbb{R}^{n \times n}$ is the system matrix, $B \in \mathbb{R}^{n \times s}$ is the matrix of input gains, the matrix $C \in \mathbb{R}^{k \times n}$ specifies the measurements of the state variables, $q_{x} \in L^{\infty}\left(\mathbb{R}, \mathbb{R}^{n}\right)$ is a system perturbation and the function $q_{y} \in L^{\infty}\left(\mathbb{R}, \mathbb{R}^{n}\right)$ models the measurement noise.

Corollary 5.3. Let the pair $\{A, C\}$ be observable then the homogeneous observer designed in Theorem 5.2 is robust in the ISS sense with respect to $q_{x}$ and $q_{y}$ provided that the matrix $I_{n}+\nu\left(G_{0}+I_{n}\right)$ is anti-Hurwitz. In other words, $\exists \xi \in \mathcal{K} \mathcal{L}$ and $\exists \theta \in \mathcal{K}$ such that

$$
\|e(t)\| \leq \xi(\|e(0)\|, t)+\theta\left(\max \left\{\left\|q_{x}\right\|_{L^{\infty}\left((0, t), \mathbb{R}^{n}\right)},\left\|q_{y}\right\|_{L^{\infty}\left((0, t), \mathbb{R}^{k}\right)}\right\}\right) \text {. }
$$

This result immediately follows from Theorem 3.11. 


\subsection{Homogeneous differentiator}

Consider a nonlinear system in the observable canonical form:

$$
\begin{aligned}
\dot{x} & =A x+f(y, u), \\
y & =C x,
\end{aligned}
$$

where $x \in \mathbb{R}^{n}$ is the state, $u \in \mathbb{R}^{m}$ is the control input, $y \in \mathbb{R}$ is the measured output, $f \in \mathbb{R}^{m+1} \rightarrow \mathbb{R}^{n}$ is a known nonlinear function ensuring existence and uniqueness of solutions at least locally in time, and

$$
A=\left(\begin{array}{ccccc}
0 & 1 & 0 & 0 & 0 \\
0 & 0 & 1 & 0 & 0 \\
\vdots & \vdots & \vdots & \ddots & \vdots \\
0 & 0 & 0 & 0 & 1 \\
0 & 0 & 0 & 0 & 0
\end{array}\right), C=\left(\begin{array}{llll}
1 & 0 & \ldots & 0
\end{array}\right) .
$$

The homogeneous observer for this system (Perruquetti et al., 2008) is well-known (recall $\lceil x\rfloor^{\gamma}=|x|^{\gamma} \operatorname{sign}(x)$ for any $x \in \mathbb{R}$ and $\gamma \in \mathbb{R}_{+}$):

$$
\dot{\hat{x}}=A \hat{x}+f(y, u)+\left(\begin{array}{c}
k_{1}\left\lceil y-\hat{x}_{1}\right\rfloor^{\alpha_{1}} \\
k_{2}\left\lceil y-\hat{x}_{1}\right\rfloor^{\alpha_{2}} \\
\vdots \\
k_{n}\left\lceil y-\hat{x}_{1}\right\rfloor^{\alpha_{n}}
\end{array}\right),
$$

where $\hat{x} \in \mathbb{R}^{n}$ is the state $x$ estimate, $k_{1}, \ldots, k_{n} \in \mathbb{R}$ are observer gains, and the powers $\alpha_{i}=1+i \nu, 1 \leq i \leq n$ for some $\nu \in\left(-\frac{1}{n}, 0\right)$ are selected in a way to guarantee that the estimation error $e=x-\hat{x}$ dynamics,

$$
\begin{aligned}
\dot{e}_{1} & =e_{2}-k_{1}\left\lceil e_{1}\right\rfloor^{1+\nu}, \\
\dot{e}_{2} & =e_{3}-k_{2}\left\lceil e_{1}\right\rfloor^{1+2 \nu}, \\
& \vdots \\
\dot{e}_{n} & =-k_{n}\left\lceil e_{1}\right\rfloor^{1+n \nu},
\end{aligned}
$$

is homogeneous of degree $\nu$ with respect to the weights $r_{i}=(i-1) \nu+1$, $1 \leq i \leq n$. 
Theorem 5.4 (Perruquetti et al., 2008). For any coefficients $k_{1}, \ldots, k_{n}$ forming a Hurwitz polynomial there exists $\nu \in\left(-\frac{1}{n}, 0\right)$ (sufficiently close to 0 ) such that (5.14) is globally finite-time stable.

Using the properties of homogeneous systems it is possible to show that (5.14) is input-to-state stable with respect to additive perturbations and output measurable noises (Bernuau et al., 2013), and for any delay in the measurement channel the estimation error boundedness is ensured (Zimenko et al., 2017).

Using similar arguments the case of $\nu>0$ can be considered:

Corollary 5.5. For any coefficients $k_{1}, \ldots, k_{n}$ forming a Hurwitz polynomial there exists $\nu>0$ (sufficiently close to 0 ) such that (5.14) is globally nearly fixed-time stable.

For $f(y, u)=0$, the homogeneous finite-time observer (5.13) is frequently used as a differentiator, whose aim is to estimate the derivatives of the signal $y$. In such a case, $\hat{x}_{i}$ can be utilized as an estimate of $y^{(i-1)}$ derivative for $1 \leq i \leq n$ (here $y^{(0)}=y$ ). If $\nu=-\frac{1}{n}$, then this differentiator has a discontinuity in the last line, i.e., $\left\lceil e_{1}\right\rfloor^{1+n \nu}=\operatorname{sign}\left(e_{1}\right)$, and it is a very popular differentiation algorithm called High Order Sliding Mode (HOSM) differentiator of Levant, 2005. If $\nu=0$, then (5.13) becomes a well-known linear high-gain observer (Khalil, 2002). 
Part III

\section{Discretizations and extensions to infinite dimensional systems}


All previously presented results about analysis of finite-time and fixed-time stability and convergence properties, or about design of control and estimation algorithms possessing accelerated convergence rates, have been given for continuous-time models. However, if these algorithms have to be realized in digital controllers, for simulations, or in the presence of communication networks, then the considered models should include discrete-time components, time delays or eventbased procedures. As we are going to show in this part, appearance of delays (their can be used to represent all mentioned previously phenomena) drastically changes the abilities of the systems with nonasymptotic convergence. This observation admits a simple intuition: finite-/fixed-time convergences are related with non-Lipschitz behavior and high-gains, and these kinds of dynamics are highly sensitive to any kinds of lags.

This part has two chapters. In Chapter 6, various aspects of discretization of considered finite-time and fixed-time converging homogeneous systems are investigated, and it is demonstrated that the methods of approximation of solutions of this kind of dynamics have to be carefully selected and developed in order to recover the established convergence rates in the discrete time. In Chapter 7, this discussion is extended by analysis of time-delay systems, and it is concluded by the study of partial differential equations. 


\section{Implementation and discretization}

Usually, for a continuous-time system, after analysis or design have been performed, for verification or implementation, the system solutions have to be calculated in a computer or in a digital controller (e.g., for a state observer). For these purposes, different numerical approximation methods and discretization schemes are used (Allen and Isaacson, 1998; Butcher, 2008). For example, the Euler method is a first-order numerical routine for solving ordinary differential equations with a given initial value and time step, which represents the most basic explicit/implicit method of numerical integration and it is the simplest Runge-Kutta method.

\subsection{Discretization of homogeneous dynamics}

The applicability conditions of the most discretization approaches are obtained for locally Lipschitz systems having frequently a local nature. Considering finite-time or fixed-time stable dynamics we are oblige to deal with non-Lipschitz cases and global comportment. Since homogeneous systems represent a useful case study for finite-time or fixed-time convergences, in this section the implementation issues of stronger-thanasymptotically converging systems and derivation of their solutions will 
be analyzed for this class of models. First, the applicability of the most popular Euler method will be analyzed. Next, improvements will be presented, which can be obtained by developing consistent discretization tools or state-dependent step methods. Finally, the features of discretization of ILF control and estimation algorithms will be highlighted.

\subsubsection{Explicit and implicit Euler methods}

The conditions of convergence and stability of the explicit and implicit Euler methods have been studied for linear systems (the notion of A-stability (Butcher, 2008; Dahlquist, 1963)), or for some classes of nonlinear ones. For homogeneous systems it has been shown that application of the explicit Euler method for the global approximation of solutions of homogeneous systems with non-zero degree is problematic (Levant, 2013; Efimov et al., 2017), and the implicit Euler scheme has a better perspective (Acary and Brogliato, 2010; Brogliato and Polyakov, 2015; Huber et al., 2016; Miranda-Villatoro et al., 2017). It is worth stressing that the implicit Euler method has higher computational complexity than the explicit one. Let us present the main statements of these results.

Consider the following nonlinear system:

$$
\dot{x}(t)=f(x(t)), t \geq 0,
$$

where $x(t) \in \mathbb{R}^{n}$ is the state, $f: \mathbb{R}^{n} \rightarrow \mathbb{R}^{n}$ ensures forward existence and uniqueness of the system solutions at least locally (if $f$ is discontinuous, then the solutions are understood in the Filippov's sense (Filippov, 1988)), $f(0)=0$. For an initial condition $x_{0} \in \mathbb{R}^{n}$ define the corresponding solution by $X\left(t, x_{0}\right)$ for any $t \geq 0$ for which the solution exists.

In order to approximate solution $X\left(t, x_{0}\right)$ of the system (6.1) for some initial state $x_{0} \in \mathbb{R}^{n}$, select a discretization step $h>0$, define a sequence of time instants $t_{i}=i h$ for $i=0,1, \ldots$, and denote by $x_{i}$ an approximation of the solution $X\left(t_{i}, x_{0}\right)$ at the corresponding time instant (i.e., $x_{i} \simeq X\left(t_{i}, x_{0}\right)$ and $x_{0}=x\left(t_{0}\right)=x(0)$ ), then the approximation $x_{i+1}$ calculated in accordance with the explicit Euler 
method is given by (Butcher, 2008):

$$
x_{i+1}=x_{i}+h f\left(x_{i}\right)
$$

for $i=0,1, \ldots$, while the approximation calculated by the implicit Euler method comes from (Butcher, 2008):

$$
x_{i+1}=x_{i}+h f\left(x_{i+1}\right)
$$

for $i=0,1, \ldots$ In the sequel, the problem of convergence to zero of the approximations $\left\{x_{i}\right\}_{i=0}^{\infty}$ derived in (6.2) and (6.3) is studied for the system (6.1) admitting the following hypothesis:

Assumption 6.1. Let (6.1) be $\mathbf{d}$-homogeneous with a degree $\nu \in \mathbb{R}$ and asymptotically stable.

To proceed we need to establish some properties of solutions in (6.2) and (6.3).

\section{Existence of approximations and their relations}

Existence of some $x_{i+1} \in \mathbb{R}^{n}$ for any $x_{i} \in \mathbb{R}^{n}$ in the explicit case (6.2) is straightforward, but it is not the case of (6.3). From homogeneity property we can obtain the following result:

Proposition 6.1. (Efimov et al., 2017) Let system (6.1) be $\mathbf{d}$-homogeneous with a degree $\nu \neq 0$. Let for any $x_{0} \in S$ and all $h>0$ there exist a sequence $\left\{x_{i}\right\}_{i=0}^{\infty}$ obtained by (6.2) or (6.3) with initial state $x_{0}$. Then for any discretization step $h^{\prime}>0$ and for any $y_{0} \in \mathbb{R}^{n}$ there exist a sequence $\left\{y_{i}\right\}_{i=0}^{\infty}$ generated by (6.2) or (6.3) with the step $h^{\prime}$ and the initial state $y_{0}$.

Note that the above result does not provide a conclusion about boundedness or convergence of the obtained sequences.

In the general case, it is difficult to provide some simple conditions for existence and uniqueness of solution of the implicit Euler method, but homogeneity may simplify the analysis as usual: 
Proposition 6.2. (Efimov et al., 2017) If the function $f: \mathbb{R}^{n} \rightarrow \mathbb{R}^{n}$ is continuously differentiable outside the origin, $\mathbf{d}$-homogeneous of degree $\nu \neq 0$ and there exists $h_{0}>0$ such that

$$
\operatorname{det}\left(I_{n}-h_{0} \frac{\partial f(x)}{\partial x}\right) \neq 0 \quad \forall x \in \mathbb{R}^{n} \backslash\{0\},
$$

then for $n \geq 2$ the equation (6.3) has a solution with respect to $x_{i+1} \in \mathbb{R}^{n}$ for any $x_{i} \in \mathbb{R}^{n}$ and for any $h>0$, additionally, for $n \geq 3$ the solution is unique.

Due to homogeneity there are relations between the approximations obtained for different initial conditions and discretization steps:

Proposition 6.3. (Efimov et al., 2017) Let system (6.1) be $\mathbf{d}$-homogeneous with a degree $\nu \in \mathbb{R}$. If $\left\{x_{i}\right\}_{i=0}^{\infty}$ is a sequence generated by (6.2) or (6.3) with the step $h$ and the initial state $x_{0}$, then for any $s \in \mathbb{R}$, $y_{i}=\mathbf{d}(s) x_{i}$ is a sequence obtained by (6.2) or (6.3), respectively, with the step $e^{-\nu s} h$ and the initial state $y_{0}=\mathbf{d}(s) x_{0}$.

Note that $y_{i}$ is an approximation of $X\left(e^{-\nu s} h i, y_{0}\right)$ for shifted instants of time. The following corollaries can be established.

Corollary 6.1. (Efimov et al., 2017) Let system (6.1) be d-homogeneous with a degree $\nu=0$. Let for all $x_{0} \in S$ there exist sequences $\left\{x_{i}\right\}_{i=0}^{\infty}$ obtained by (6.2) or (6.3) with the step $h>0$ and the initial state $x_{0}$ possessing one of the following properties:

$$
\begin{gathered}
\sup _{i \geq 0}\left\|x_{i}\right\|<+\infty ; \\
\lim _{i \rightarrow+\infty} x_{i}=0 .
\end{gathered}
$$

Then for any $y_{0} \in \mathbb{R}^{n}$ there exist sequences $\left\{y_{i}\right\}_{i=0}^{\infty}$ generated by (6.2) or (6.3) with the step $h$ and the initial state $y_{0}$ possessing the same property. 
Corollary 6.2. (Efimov et al., 2017) Let system (6.1) be d-homogeneous with a degree $\nu \neq 0$. Let there exist $h>0$ such that for any $x_{0} \in S$ the sequences $\left\{x_{i}\right\}_{i=0}^{\infty}$ obtained by (6.2) or (6.3) with the step $h$ and the initial state $x$ possess one of the properties (6.5), (6.6). Then for any $y_{0} \in \mathbb{R}^{n}$ the sequences $\left\{y_{i}\right\}_{i=0}^{\infty}$ obtained by (6.2) or (6.3) with the step $h\left\|y_{0}\right\|_{\mathbf{d}}^{-\nu}$ and the initial state $y_{0}$ possess the same property.

The results of corollaries 6.1 and 6.2 show advantages and limitations of the Euler method application for calculation of solutions of homogeneous systems with different degrees. For the case $\nu=0$ the properties of approximation $x_{i}$ depend on size of the step $h$, while for $\nu \neq 0$ if a scheme provides approximation of solutions for some $h$, then similar properties can be obtained for any initial condition with a properly scaled step $h^{\prime}$.

Corollary 6.3. (Efimov et al., 2017) Let system (6.1) be d-homogeneous with a degree $\nu \neq 0$. Let for any $x_{0} \in \mathbb{R}^{n}$ and some $h>0$ there exist sequences $\left\{x_{i}\right\}_{i=0}^{\infty}$ obtained by (6.2) or (6.3) with initial state $x_{0}$ possessing one of the properties (6.5), (6.6). Then for any discretization step $h^{\prime}>0$ and for any $y_{0} \in \mathbb{R}^{n}$ there exist sequences $\left\{y_{i}\right\}_{i=0}^{\infty}$ generated by (6.2) or (6.3) with the step $h^{\prime}$ and the initial state $y_{0}$ possessing the same property.

Thus, for $\nu \neq 0$ if a scheme provides approximation of solutions globally for some $h$, then similar properties can be obtained for any step $h^{\prime}$. The latter characteristic is unlikely in general, thus using only homogeneity the global result for the case $\nu \neq 0$ cannot be obtained for (6.2) or (6.3).

\section{Convergence of sequences $\left\{x_{i}\right\}_{i=0}^{\infty}$ generated by Euler methods}

Since (6.1) is homogeneous and asymptotically stable under Assumption 6.1 , there is a twice continuously differentiable and $\mathbf{d}$-homogeneous 
Lyapunov function $V: \mathbb{R}^{n} \rightarrow \mathbb{R}_{+}$of positive degree $\mu>-\nu$ such that

$$
\begin{gathered}
a=-\sup _{\xi \in S} L_{f} V(\xi)>0, \\
0<b=\sup _{\|\xi\|_{\mathbf{d}} \leq 1}\left\|\frac{\partial V(\xi)}{\partial \xi}\right\|<+\infty, \\
c_{1}=\inf _{\xi \in S} V(\xi), c_{2}=\sup _{\xi \in S} V(\xi), \\
c_{1}\|x\|_{\mathbf{d}}^{\mu} \leq V(x) \leq c_{2}\|x\|_{\mathbf{d}}^{\mu} \quad \forall x \in \mathbb{R}^{n} .
\end{gathered}
$$

Let us take the discretization step $h>0$ and consider the behavior of $V$ from (6.7) on a sequence generated by (6.2). For this purpose define $x_{i}=\mathbf{d}(s) y_{i}$ with $y_{i} \in S$ and $s=\ln \left\|x_{i}\right\|_{\mathbf{d}}$ :

$$
\begin{gathered}
V\left(x_{i+1}\right)-V\left(x_{i}\right)=V\left(x_{i}+h f\left(x_{i}\right)\right)-V\left(x_{i}\right) \\
=e^{\mu s}\left[V\left(y_{i}+e^{\nu s} h f\left(y_{i}\right)\right)-V\left(y_{i}\right)\right]=e^{(\nu+\mu) s} h \frac{\partial V(\xi)}{\partial \xi} f\left(y_{i}\right)
\end{gathered}
$$

for $\xi=y_{i}+e^{\nu s} \varrho f\left(y_{i}\right)$ with $\varrho \in[0, h]$ and the Mean Value Theorem has been used on the last step. Note that

$$
\underline{\sigma}\left(\|\xi\|_{\mathbf{d}}\right) \leq\|\xi\| \leq\left\|y_{i}\right\|+\left\|x_{i}\right\|_{\mathbf{d}}^{\nu} \varrho\left\|f\left(y_{i}\right)\right\| \leq \bar{\sigma}(1)+g\left\|x_{i}\right\|_{\mathbf{d}}^{\nu} h
$$

for $g=\sup _{y \in S}\|f(y)\|$ and some $\underline{\sigma}, \bar{\sigma} \in \mathcal{K}_{\infty}$. Next,

$$
\begin{gathered}
V\left(x_{i+1}\right)-V\left(x_{i}\right)=e^{(\nu+\mu) s} h\left\{\frac{\partial V\left(y_{i}\right)}{\partial y_{i}} f\left(y_{i}\right)\right. \\
\left.+\frac{\partial V(\xi)}{\partial \xi} f\left(y_{i}\right)-\frac{\partial V\left(y_{i}\right)}{\partial y_{i}} f\left(y_{i}\right)\right\} \\
\leq h e^{(\nu+\mu) s}\left(-a+g\left\|\frac{\partial V(\xi)}{\partial \xi}-\frac{\partial V\left(y_{i}\right)}{\partial y_{i}}\right\|\right) .
\end{gathered}
$$

Since $\left\|\frac{\partial V(\xi)}{\partial \xi}-\frac{\partial V\left(y_{i}\right)}{\partial y_{i}}\right\| \leq k\left\|\xi-y_{i}\right\|$ where $k>0$ is the Lipschitz constant of $\frac{\partial V(\xi)}{\partial \xi}$ on the set $\xi \in\left\{\xi \in \mathbb{R}^{n}:\|\xi\|_{\mathbf{d}} \leq \underline{\sigma}^{-1}\left(\bar{\sigma}(1)+g\left\|x_{i}\right\|_{\mathbf{d}}^{\nu} h\right)\right\}$, then

$$
\begin{gathered}
V\left(x_{i+1}\right)-V\left(x_{i}\right) \leq h e^{(\nu+\mu) s}\left\{-a+g k\left\|\xi-y_{i}\right\|\right\} \\
\leq h e^{(\nu+\mu) s}\left\{-a+g k e^{\nu s} \varrho\left\|f\left(y_{i}\right)\right\|\right\} \leq h e^{(\nu+\mu) s}\left\{-a+g^{2} k e^{\nu s} h\right\} .
\end{gathered}
$$

Therefore, the condition of convergence for (6.2) is

$$
e^{\nu s} h<\frac{a}{g^{2} k},
$$


where in the right-hand side all constants are independent on the discretization approach. If (6.8) is satisfied, then $V\left(x_{i+1}\right)<V\left(x_{i}\right)$, or $\left\|x_{i+1}\right\|_{\mathbf{d}}<\left(c_{1}^{-1} c_{2}\right)^{1 / \mu}\left\|x_{i}\right\|_{\mathbf{d}}$.

For the implicit scheme (6.3) exactly the same calculations can be repeated showing that $V\left(x_{i+1}\right)<V\left(x_{i}\right)$ under (6.8), then the following results are obtained:

Theorem 6.4. (Efimov et al., 2017) Let Assumption 6.1 hold for $\nu=0$, then there exists the discretization step $h>0$ such that the sequences $\left\{x_{i}\right\}_{i=0}^{\infty}$ obtained by (6.2) or (6.3) for any initial state $x_{0} \in \mathbb{R}^{n}$ and the step $h$ possess the following properties:

(a) $\left\|x_{i}\right\|_{\mathbf{d}}<\gamma\left\|x_{0}\right\|_{\mathbf{d}}$ for all $i \geq 0$ for some $\gamma \geq 1$;

(b) $\lim _{i \rightarrow+\infty} x_{i}=0$.

Note that for $\nu=0$ the discrete-time systems are homogeneous in the sense of (Tuna and Teel, 2004), (Sanchez et al., 2019).

Theorem 6.5. (Efimov et al., 2017) Let Assumption 6.1 hold for $\nu<0$, then for any $\rho>0$ there exists a discretization step $h_{\rho}>0$ such that the sequences $\left\{x_{i}\right\}_{i=0}^{\infty}$ obtained by (6.2) or (6.3) for any initial state $x_{0} \in\left\{x \in \mathbb{R}^{n}:\|x\|_{\mathbf{d}}>\rho\right\}$ with a step $h \leq h_{\rho}$ possess the following properties:

(a) $\left\|x_{i}\right\|_{\mathbf{d}}<\gamma\left\|x_{0}\right\|_{\mathbf{d}}$ for all $i \geq 0$ for some $\gamma \geq 1$;

(b) there exists $i_{x_{0}}>0$ such that $x_{i_{x_{0}}} \in\left\{x \in \mathbb{R}^{n}:\|x\|_{\mathbf{d}} \leq \rho\right\}$.

As follows from Theorem 6.5, in the case $\nu<0$, for any $h>0$ the Euler schemes provide the global convergence into some vicinity of the origin, and this vicinity is shrinking as $h \rightarrow 0$ (the radius of the vicinity is proportional to $h^{-1 / \nu}$ (Levant, 2005)).

Theorem 6.6. (Efimov et al., 2017) Let Assumption 6.1 hold for $\nu>0$, then for any $\rho>0$ there exists a discretization step $h_{\rho}>0$ such that the sequences $\left\{x_{i}\right\}_{i=0}^{\infty}$ obtained by (6.2) or (6.3) for any initial state $x_{0} \in\left\{x \in \mathbb{R}^{n}:\|x\|_{\mathbf{d}} \leq \rho\right\}$ with a step $h \leq h_{\rho}$ possess the following properties: 
(a) $\left\|x_{i}\right\|_{\mathbf{d}}<\gamma\left\|x_{0}\right\|_{\mathbf{d}}$ for all $i \geq 0$ for some $\gamma \geq 1$;

(b) $\lim _{i \rightarrow+\infty} x_{i}=0$.

According to Theorem 6.6, in the case $\nu>0$, for any $h>0$ the Euler schemes preserve the asymptotic convergence to zero locally, and the domain of convergence goes global as $h \rightarrow 0$ (it can be shown that the radius $\rho$ is proportional to $h^{-1 / \nu}$ ).

More advantageous conditions for (6.3) can be obtained by imposing some additional but mild restrictions (we also assume that solutions exists, i.e., the conditions of Proposition 6.5 are satisfied):

Theorem 6.7. (Efimov et al., 2017) Let Assumption 6.1 hold and $V: \mathbb{R}^{n} \rightarrow \mathbb{R}_{+}$be a continuously differentiable $\mathbf{d}$-homogeneous Lyapunov function of degree $\mu$ for the system (6.1). Then for the sequence $\left\{x_{i}\right\}_{i=0}^{\infty}$ generated by the implicit scheme (6.3) with any step $h>0$ and any $x_{0} \in \mathbb{R}^{n}$, the sequence $\left\{V\left(x_{i}\right)\right\}_{i=1}^{+\infty}$ is monotonously decreasing to zero provided that

$$
\langle x-y, f(x)\rangle \neq\|x-y\| \cdot\|f(x)\|
$$

for all $x \neq y$ such that $x, y \in\left\{z \in \mathbb{R}^{n}: V(z)=1\right\}$.

It is easy to show that if the level set of the Lyapunov function $V$ is convex, then the condition (6.9) of this theorem holds. Note that a time-varying step can be used in the conditions of Theorem 6.7.

It was also proven in (Efimov et al., 2017) that the explicit Euler scheme (6.2) is divergent for small or big enough initial conditions for the cases $\nu<0$ or $\nu>0$, respectively.

\section{Absolute and Relative Errors of Discretized Homogeneous Systems}

Standard characteristics of any discretization routine include its precision: an error between the obtained solution approximation $x_{i}$ and the solution itself $X\left(i h, x_{0}\right)$. In order to evaluate precision of Euler schemes for homogeneous systems, denote by $x_{i+1}\left(h, x_{i}\right)$ the value derived by (6.2) or (6.3) for $x_{i}$ and $h>0$, then (Dahlquist and Björck, 2008) 
- absolute error is the magnitude of the difference between the exact value and its approximation:

$$
\Delta\left(h, x_{i}\right)=\left\|X\left(h, x_{i}\right)-x_{i+1}\left(h, x_{i}\right)\right\|_{\mathbf{d}} ;
$$

- relative error expresses how large the absolute error is compared with the exact value:

$$
\delta\left(h, x_{i}\right)=\frac{\Delta\left(h, x_{i}\right)}{\left\|X\left(h, x_{i}\right)\right\|_{\mathbf{d}}} .
$$

The errors are defined in the homogeneous norm $\|\cdot\|_{\mathbf{d}}$, equivalence of such a formulation and the one based on the conventional norm $\|\cdot\|$ was demonstrated in (Efimov et al., 2017). The proposed quantities with $\|\cdot\|_{\mathbf{d}}$ suit better for analysis of homogeneous systems:

Theorem 6.8. (Efimov et al., 2017) Let the system (6.1) be $\mathbf{d}$-homogeneous of degree $\nu$ and $x_{i+1}\left(h, x_{i}\right)$ be calculated by the explicit (6.2) or implicit (6.3) Euler scheme for $x_{i} \in \mathbb{R}^{n}$ and $h>0$. Then

1) $\Delta\left(h, \mathbf{d}(s) x_{i}\right)=e^{s} \Delta\left(h e^{\nu s}, x_{i}\right)$ and $\delta\left(h, \mathbf{d}(s) x_{i}\right)=\delta\left(h e^{\nu s}, x_{i}\right)$ for any $h>0$ and $x_{i} \neq 0$;

2) $\delta\left(h, x_{i}\right) \rightarrow 0$ as $x_{i} \rightarrow \infty$ if $\nu<0$

3) $\delta\left(h, x_{i}\right) \rightarrow 0$ as $x_{i} \rightarrow 0$ if $\nu>0$.

If, in addition, the system (6.1) is asymptotically stable then

4) $\delta\left(h, x_{i}\right) \rightarrow \infty$ as $x_{i} \rightarrow 0$ if $\nu<0$;

5) $\delta\left(h, x_{i}\right) \rightarrow \infty$ as $x_{i} \rightarrow \infty$ if $\nu>0$.

Therefore, for any value of the discretization step, the explicit and implicit Euler schemes provide a good approximation (i.e., small relative error $\delta$ ) of the system solutions if $\nu<0$ for big values of initial conditions, and if $\nu>0$ in a vicinity of the origin. Roughly speaking, if a homogeneous system has a slower rate of convergence than a linear one (far outside of the origin for $\nu<0$ or in a neighborhood of the origin for $\nu>0$ ), then the Euler methods ensure a good precision. 
Example 6.1. Consider a scalar stable linear system

$$
\dot{x}=-x,
$$

which is homogeneous of degree $\nu=0$ for $\mathbf{d}(s)=e^{s}$. Its discretizations (6.2) and (6.3) can be written as follows:

$$
\begin{gathered}
x_{i+1}=(1-h) x_{i}, \\
x_{i+1}=(1+h)^{-1} x_{i} .
\end{gathered}
$$

Thus, the scheme (6.3) is always converging in this example (by Theorem 6.7 , since the system has a Lyapunov function $V=x^{2}$ for which the condition (6.9) is satisfied), but (6.2) is diverging for any $h>2$ (an illustration for Corollary 6.1).

Example 6.2. Consider a scalar stable nonlinear system

$$
\dot{x}=-|x| x,
$$

which is homogeneous of degree $\nu=1$ for $\mathbf{d}(s)=e^{s}$, with its corresponding discretizations (6.2) and (6.3):

$$
\begin{gathered}
x_{i+1}=\left(1-h\left|x_{i}\right|\right) x_{i}, \\
x_{i+1}=\frac{1}{2 h}\left(\sqrt{4 h\left|x_{i}\right|+1}-1\right) \operatorname{sign}\left(x_{i}\right) .
\end{gathered}
$$

Then the explicit scheme (6.2) is converging for any $h>0$ with $\left|x_{i}\right|<$ $2 h^{-1}$ (Theorem 6.6), while the implicit one (6.3) is converging globally (by Theorem 6.7 with $V=x^{2}$ ).

Example 6.3. Consider another scalar stable nonlinear system

$$
\dot{x}=-|x|^{0.5} \operatorname{sign}(x),
$$

which is homogeneous of degree $\nu=-0.5$ for $\mathbf{d}(s)=e^{s}$, with its corresponding discretizations (6.2) and (6.3):

$$
\begin{gathered}
x_{i+1}=\left(1-h\left|x_{i}\right|^{-0.5}\right) x_{i}, \\
x_{i+1}=\frac{1}{4}\left(\sqrt{4\left|x_{i}\right|+h^{2}}-h\right)^{2} \operatorname{sign}\left(x_{i}\right) .
\end{gathered}
$$

Then the explicit scheme (6.2) is converging for any $h>0$ with $\left|x_{i}\right|>$ $0.25 h^{2}$ (Theorem 6.5), while the implicit one (6.3) is converging globally (again, by Theorem 6.7 with $V=x^{2}$ ). 


\section{Discussion}

For the case of $\nu=0$ the properties of approximations are dependent on the discretization step $h$, and convergence to zero of the Euler schemes for one value of the step does not imply the same property for another one (Theorem 6.4). However, for the case $\nu \neq 0$, convergence to the origin or boundedness of approximations obtained for some step on a sphere implies the same property for a properly selected discretization step for any initial condition (Corollary 6.2). In the case of $\nu<0$ it has been proven that the approximations globally converge to some vicinity of the origin (Theorem 6.5). For the case $\nu>0$, it has been proven that for sufficiently small steps the approximations locally converge in some vicinity of the origin (Theorem 6.6). The attracting neighborhood of the origin for $\nu<0$ or the domain of attraction for $\nu>0$ can be contracted to 0 or enlarged to infinity, respectively, as the step tends to 0 .

For the implicit Euler scheme, under additional mild conditions, it has been proven that for any initial conditions and discretization steps the solutions always exist and asymptotically converge to zero (Proposition 6.5 and Theorem 6.7). However, the implicit Euler method has a higher computational complexity than the explicit one. For $\nu<0$ the explicit Euler method can be used outside of a vicinity of the origin and next switching to the implicit Euler methods is reasonable, in order to demonstrate convergence to the origin (initial application of the explicit method is motivated by its lower computational complexity).

To conclude, the conventional Euler methods do not keep the accelerated convergence rates in discrete time, then other discretization approaches have to be developed for homogeneous systems with nonzero degrees.

\subsubsection{Consistent discretization}

\section{Motivating examples}

Example 6.4. Inspired by (Polyakov et al., 2019) let us consider the following homogeneous systems:

$$
\dot{x}=-2 \sqrt{|x|} \overline{\operatorname{sign}}(x), \quad y=\sqrt{|x|} \stackrel{\overline{\operatorname{sign}}}{\Leftrightarrow}(x) \quad \dot{y} \in-\overline{\operatorname{sign}}(y),
$$


where

$$
\overline{\operatorname{sign}}(\rho)=\left\{\begin{array}{ccc}
1 & \text { if } & \rho>0 \\
{[-1,1]} & \text { if } & \rho=0 \\
-1 & \text { if } & \rho<0
\end{array}\right.
$$

These systems are standard homogeneous, finite-time stable and topologically equivalent (homeomorphic on $\mathbb{R}$ and diffeomorphic on $\mathbb{R} \backslash\{\mathbf{0}\}$ ). Indeed, if $x\left(\cdot, x_{0}\right)$ is the solution of the first system with $x(0)=x_{0} \in \mathbb{R}$ then $y\left(\cdot, y_{0}\right)=\sqrt{\left|x\left(\cdot, x_{0}\right)\right|} \operatorname{sign}\left(x\left(\cdot, x_{0}\right)\right)$ is the solution of the second system with $y(0)=y_{0}=\sqrt{\left|x_{0}\right|} \operatorname{sign}\left(x_{0}\right)$, and vice versa.

The implicit Euler discretizations of these systems are given by:

$$
x_{i+1}=x_{i}-2 h \sqrt{\left|x_{i+1}\right|} \operatorname{sign}\left(x_{i+1}\right) \quad \Leftrightarrow \quad \begin{aligned}
& y_{i+1}=y_{i}+h \tilde{u}_{i} \\
& \tilde{u}_{i} \in-\overline{\operatorname{sign}}\left(y_{i+1}\right)
\end{aligned}
$$

where $h>0$ is the sampling period, $x_{i}=x\left(i h, x_{0}\right), y_{i}=y\left(i h, y_{0}\right)$ for $t \in[i h,(i+1) h)$, and $\tilde{u}_{i}$ is an auxiliary variable, $i=0,1,2 \ldots$ We refer the reader to (Acary et al., 2012) for more details about the implicit discretization of the discontinuous system.

The discretization destroys the topological equivalence between systems, since

$$
x_{i+1}=\left(\sqrt{h^{2}+\left|x_{i}\right|}-h\right)^{2} \operatorname{sign}\left(x_{i}\right), \Leftrightarrow y_{i+1}=\left\{\begin{array}{cl}
y_{i}-h \operatorname{sign}\left(y_{i}\right) & \text { if }\left|y_{i}\right|>h, \\
0 & \text { if }\left|y_{i}\right| \leq h,
\end{array}\right.
$$

Indeed, the discrete-time approximation of the first system is just asymptotically stable $\left(x_{0} \neq 0 \Rightarrow x_{i} \neq 0, \forall i\right)$, but the discretization of the second equation remains finite-time stable $\left(\exists i^{*}=i^{*}\left(y_{0}\right): y_{i}=\right.$ $\left.0, \forall i \geq i^{*}\right)$. A continuous invertible coordinate transformation, which transforms a solution set of the first discrete-time system to a solution set of the second one, does not exist.

Moreover, the discrete-time approximation of the first (continuous) homogeneous system is inconsistent with its continuous-time counterpart in the context of convergence rates. A reasonable way to discretize it consistently is to use the equivalence with the second system. Using solutions of the consistently discretized (second) system we can recover the finite-time convergent solutions of the first dynamics by means of the posterior coordinate transformation $\hat{x}_{i}=y_{i}^{2} \operatorname{sign}\left(y_{i}\right)$. The suggested 
approach gives the following approximation

$$
\hat{x}_{i+1}=\left\{\begin{array}{cll}
\left(\sqrt{\left|\hat{x}_{i}\right|}-h\right)^{2} \operatorname{sign}\left(\hat{x}_{i}\right) & \text { if } & \left|\hat{x}_{i}\right|>h^{2}, \\
0 & \text { if } & \left|\hat{x}_{i}\right| \leq h^{2},
\end{array}, \quad \tilde{x}_{0}=x_{0}\right.
$$

which is, obviously, finite-time stable. Any stable continuous homogeneous system of a degree $\mu<0$ admits a consistent discrete-time approximation that preserves the finite-time convergence of all trajectories to the origin.

Example 6.5. Again inspired by (Polyakov et al., 2019) let us consider the scalar system

$$
\dot{x}=-|x| x,
$$

which is globally nearly fixed-time stable: $\left|x\left(t, x_{0}\right)\right|<\varepsilon$ for $t>\frac{1}{\varepsilon}$ independently of the initial state $x_{0}$.

The explicit Euler method applied to the system (6.10) gives the discrete-time model

$$
x_{i+1}=x_{i}-h\left|x_{i}\right| x_{i}
$$

It has solutions which blow up if $h>2 /\left|x_{0}\right|$, i.e. the discrete-time approximation is not globally stable.

The implicit Euler discretization yields the globally asymptotically stable system

$$
x_{i+1}=\frac{\sqrt{1+4 h\left|x_{i}\right|}-1}{2 h} \operatorname{sign}\left(x_{i}\right),
$$

which does not preserve near fixed-time stability. Thus, it is also inconsistent with the original continuous-time model in the context of the decay rate.

Using a semi-implicit Euler discretization we derive

$$
\frac{x_{i+1}-x_{i}}{h}=-\left|x_{i}\right| x_{i+1}
$$

or, equivalently,

$$
x_{i+1}=\frac{x_{i}}{1+h\left|x_{i}\right|} .
$$

It is easy see that $\left|x_{1}\right| \leq(h)^{-1}$ independently of $x_{0}$, and

$$
\begin{gathered}
\left|x_{2}\right|=\frac{1}{\left|x_{1}\right|^{-1}+h} \leq \frac{1}{h+h}=(2 h)^{-1} \\
\left|x_{3}\right|=\frac{1}{\left|x_{2}\right|^{-1}+h} \leq \frac{1}{2 h+h}=(3 h)^{-1},
\end{gathered}
$$




$$
\left|x_{i}\right| \leq(i h)^{-1}
$$

i.e., the obtained discrete-time approximation remains nearly fixedtime stable. In fact, this approach works for any stable $\mathbf{d}$-homogeneous system with a positive degree.

\section{Finite-time and Fixed-time stable consistent discretizations}

Let us consider a nonlinear system

$$
\dot{x}=f(x), \quad t>0, \quad x(0)=x_{0},
$$

where $x(t) \in \mathbb{R}^{n}$ is the system state and the nonlinear function $f$ : $\mathbb{R}^{n} \rightarrow \mathbb{R}^{n}$ is continuous on $\mathbb{R}^{n} \backslash\{\mathbf{0}\}$. Its solutions are understood in the sense of Filippov (see (Filippov, 1988)):

$$
\dot{x} \in F(\mathbf{0})=\bigcap_{\varepsilon>0} \overline{\operatorname{co}} f(B(\varepsilon) \backslash\{\mathbf{0}\}),
$$

where $\overline{c o}$ denotes a closed convex hull. In our case, $F(x)=\{f(x)\}$ is a singleton for $x \in \mathbb{R}^{n} \backslash\{\mathbf{0}\}$.

Definition 6.1 (Polyakov et al., 2019). Let $Q: \mathbb{R}_{+} \times \mathbb{R}^{n} \times \mathbb{R}^{n} \rightrightarrows \mathbb{R}^{n}$ be a (possibly) set-valued mapping. The discrete-time inclusion

$$
\mathbf{0} \in Q\left(h, x_{i}, x_{i+1}\right), \quad h>0, \quad i=0,1,2, \ldots
$$

is said to be a consistent discretization (discrete-time approximation) of the globally uniformly finite-time stable system (6.11) if

1. for any $\tilde{x} \in \mathbb{R}^{n}$ and any $h>0$, there exists $\tilde{x}_{h} \in \mathbb{R}^{n}$ :

$$
\mathbf{0} \in Q\left(h, \tilde{x}, \tilde{x}_{h}\right),
$$

and $\tilde{x}_{h}=\mathbf{0}$ is the unique solution to $\mathbf{0} \in Q\left(h, \mathbf{0}, \tilde{x}_{h}\right)$.

2. for any $h>0$ each sequence

$$
\left\{x_{i}\right\}_{i=0}^{+\infty}
$$


generated by (6.13) converges to zero in a finite number of steps, i.e., for any $x_{0} \in \mathbb{R}^{n} \backslash\{\mathbf{0}\}$ there exists $i^{*}>0$ such that

$$
x_{i}=\mathbf{0} \quad \text { for } \quad i \geq i^{*}
$$

and $x_{i^{*}-1} \neq \mathbf{0}$.

3. for any $\varepsilon>0$ and any $R>\varepsilon$, there exists $\omega \in \mathcal{K}$ such that any sequence (6.15) generated by (6.13) satisfies

$$
\left\|\phi\left(h, x_{i}\right)-x_{i+1}\right\| \leq h \omega(h),
$$

provided that $\left\|x_{i+1}\right\|,\left\|x_{i}\right\| \in[\varepsilon, R]$, where $\phi\left(\cdot, x_{i}\right)$ is a solution to (6.11) with the initial condition $x(0)=x_{i}$.

The condition 6.16 guarantees that the discrete-time model (6.13) is an approximation of (6.11). Indeed, it defines the one-step discretization error and an approximation error on a time interval $[0, T]$ is $O(\omega(h))$ provided that $h=\frac{T}{N}, N \in \mathbb{N}$. This error tends to zero as $h \rightarrow 0$ (or, equivalently, $N \rightarrow+\infty$ ). Notice that the approximation errors are defined only on any compact set from $\left\{x \in \mathbb{R}^{n}: 0<\varepsilon \leq\|x\| \leq R<\right.$ $+\infty\}$ due to the singularity of the vector field $f$ at zero.

Definition 6.2 (Polyakov et al., 2019). Let $q: \mathbb{R}_{+} \times \mathbb{R}^{n} \times \mathbb{R}^{n} \rightarrow \mathbb{R}^{n}$. The equation

$$
q\left(h, x_{i}, x_{i+1}\right)=0, \quad i=0,1,2, \ldots
$$

is said to be a consistent discretization (discrete-time approximation) of the globally nearly fixed-time stable system (6.11) if it satisfies conditions 1 and 3 of Definition 6.1 and for any $r>0$ there exists $N(r)>0$ such that any sequence

$$
\left\{x_{i}\right\}_{i=0}^{+\infty}, \quad x_{0} \neq \mathbf{0}
$$

generated by the equation (6.17) satisfies

$$
\left\|x_{i}\right\| \leq r \quad \text { for } \quad i \geq N(r)
$$


independently of $x_{0}$.

\section{Results on consistent discretization}

To design a discrete-time approximation for the $\mathbf{d}$-homogeneous ODE (6.11) we use the coordinate transformation (3.22). If $f$ is $\mathbf{d}$-homogeneous of the degree -1 , then the right-hand side of the transformed system (3.9) is globally bounded. The following theorem refines the result of (Polyakov et al., 2019) allowing the condition $f(-x)=-f(x)$ to be omitted. The proof is based on Kakutani fixed-point theorem and can be found in (Polyakov, 2020).

Theorem 6.9. Let a vector field $f: \mathbb{R}^{n} \rightarrow \mathbb{R}^{n}$ be continuous on $\mathbb{R}^{n} \backslash\{\mathbf{0}\}$, d-homogeneous of the degree -1 . Let $G_{\mathbf{d}} \in \mathbb{R}^{n \times n}$ be the generator of the dilation $\mathbf{d}$ and a symmetric matrix $P \in \mathbb{R}^{n \times n}$ satisfies (3.10).

If the condition (3.12) holds with $\Xi=I_{n}$, then the mapping $Q: \mathbb{R}_{+} \times \mathbb{R}^{n} \times \mathbb{R}^{n} \rightrightarrows \mathbb{R}^{n}$ given by

$$
\begin{gathered}
Q\left(h, x_{i}, x_{i+1}\right)=\tilde{Q}\left(h, \Phi\left(x_{i}\right), \Phi\left(x_{i+1}\right)\right), \\
\Phi(x)=\|x\|_{\mathbf{d}} \mathbf{d}\left(-\ln \|x\|_{\mathbf{d}}\right) x
\end{gathered}
$$

where $h>0$ and

$$
\begin{gathered}
\tilde{Q}\left(h, y_{i}, y_{i+1}\right)=y_{i+1}-y_{i}-h \tilde{F}\left(y_{i+1}\right), \\
\tilde{F}(y)=\bigcap_{\varepsilon>0} \operatorname{co} \tilde{f}(y \dot{+} B(\varepsilon) \backslash\{\mathbf{0}\}), \\
\tilde{f}(y):=\left(\frac{\left(I-G_{\mathbf{d}}\right) y y^{\top} P}{y^{\top} G_{\mathbf{d}} P y}+I_{n}\right) f\left(\frac{y}{\sqrt{y^{\top} P y}}\right), \quad y \in \mathbb{R}^{n} \backslash\{\mathbf{0}\},
\end{gathered}
$$

defines a consistent discrete-time approximation of the system (6.11) in the sense of Definition 6.1.

The latter theorem is based on the fact that the system $\dot{y}=\tilde{f}(y)$ admits a quadratic Lyapunov function (the condition (3.12) with $\Xi=$ const). However, as it was shown in Theorem 3.10, any stable homogeneous 
system is equivalent to a quadratically stable one. If $f$ in Theorem 6.9 is replaced with the equivalent one:

$$
f^{n e w}(x)=\left.\frac{\partial \Psi(\xi)}{\partial \xi} f(\xi)\right|_{\xi=\Psi^{-1}(x)}, \quad x \in \mathbb{R}^{n}, \quad \xi \in \mathbb{R}^{n},
$$

where $\Psi \in \mathbb{F}_{\mathbf{d}}\left(\mathbb{R}^{n}\right)$ is a diffeomorphism on $\mathbb{R}^{n} \backslash\{\mathbf{0}\}$ given in Theorem 3.10 , then the condition $\Xi=I_{n}$ is fulfilled.

According to Theorem 3.10 a d-homogeneous Lyapunov function $V \in C\left(\mathbb{R}^{n}\right) \cap C^{\infty}\left(\mathbb{R}^{n} \backslash\{\mathbf{0}\}\right)$ with the degree 1 can always be found for any asymptotically stable system with a $\mathbf{d}$-homogeneous vector field $f: \mathbb{R}^{n} \rightarrow \mathbb{R}^{n}$. In this case, the required transformation $\Psi$ can be defined as follows

$$
\Psi(\xi)=\mathbf{d}\left(\ln \frac{V(\xi)}{\|\xi\|_{\mathbf{d}}}\right) \xi .
$$

Therefore, we obtain the following result.

Corollary 6.10. Any continuous d-homogeneous finite-time stable system

$$
\dot{\xi}=f(\xi)
$$

with a possible discontinuity at the origin, admits a consistent implicit approximation.

For a positive degree of homogeneity corresponding to the case of nearly fixed-time stability of the origin, we can restrict ourselves to the case of the homogeneity degree 1 without loss of generality. Notice also that the homogeneous vector field with positive degree is always continuous at the origin (see Proposition 3.1).

Theorem 6.11 (Polyakov et al., 2019). Let a vector field $f: \mathbb{R}^{n} \rightarrow$ $\mathbb{R}^{n}$ be uniformly continuous on $S, \mathbf{d}$-homogeneous of the degree 1 and, as before,

$$
\tilde{f}(z)=\left(\frac{\left(I-G_{\mathbf{d}}\right) z z^{\top} P}{z^{\top} G_{\mathbf{d}} P z}+I_{n}\right) f\left(\frac{z}{\|z\|}\right), \quad z \in \mathbb{R}^{n} \backslash\{\mathbf{0}\},
$$

where $G_{\mathbf{d}}$ is the generator of the dilation $\mathbf{d},\|z\|=\sqrt{z^{\top} P z}$ and the positive definite matrix $P \in \mathbb{R}^{n \times n}$ satisfies (3.10). If the condition 
(3.12) holds with $\Xi=I_{n}$ and

$$
S \subset W_{\alpha}\left(\mathbb{R}^{n}\right) \quad \text { for any } \quad \alpha \in(0,+\infty),
$$

where $W_{\alpha}(y):=y-\alpha\|y\| \tilde{f}(y)$, then the function $q: \mathbb{R}_{+} \times \mathbb{R}^{n} \times \mathbb{R}^{n} \rightarrow$ $\mathbb{R}^{n}$ given by

$$
q\left(h, x_{i}, x_{i+1}\right)=\tilde{q}\left(h, \Phi\left(x_{i}\right), \Phi\left(x_{i+1}\right)\right),
$$

where $h>0, \Phi(x)=\|x\|_{\mathbf{d}} \mathbf{d}\left(-\ln \|x\|_{\mathbf{d}}\right) x$ for $x \in \mathbb{R}^{n}$, and

$$
\tilde{q}\left(h, y_{i}, y_{i+1}\right)=y_{i+1}-y_{i}-h\left\|y_{i}\right\|\left\|y_{i+1}\right\| \tilde{f}\left(y_{i+1}\right)
$$

defines a consistent discrete-time approximation of the practically fixed-time stable system (6.11) in the sense of Definition 6.2.

Example 6.6 (Consistent Discretization of Fixed-Time Control in $\mathbb{R}$ ). Let us consider the scalar sliding mode control system

$$
\dot{x}=u, \quad x \in \mathbb{R}
$$

with

$$
u=\left\{\begin{array}{ccc}
-|x| x & \text { if } & |x|>1 \\
-\operatorname{sign}(x) & \text { if } & |x| \leq 1
\end{array}\right.
$$

The control is designed by means of combination of two homogeneous systems with positive and negative degrees. The closed-loop system is globally fixed-time stable, i.e., it is finite-time stable with the globally bounded settling time $T\left(x_{0}\right) \leq T^{\max }$. We have $T^{\max }=2$ for the considered scalar control system.

Theorems 6.9 and 6.11 can be directly applied to subsystems

$$
\dot{x}=-x|x|,|x|>1 \quad \text { and } \quad \dot{x}=-\operatorname{sign}(x), \quad|x| \leq 1,
$$

which are $\mathbf{d}$-homogeneous of degree -1 and 1 , respectively, with $\mathbf{d}(s)=$ $e^{G_{\mathbf{d}} s}, s \in \mathbb{R}$ and $G_{\mathbf{d}}=1$. Applying the consistent discretization schemes to these subsystems we derive

$$
x_{i+1}=\left\{\begin{array}{ccc}
\frac{x_{i}}{1+h\left|x_{i}\right|} & \text { if } & \left|x_{i}\right| \geq 1, \\
x_{i}-h \operatorname{sign}\left(x_{i}\right) & \text { if } & h<\left|x_{i}\right|<1, \\
0 & \text { if } & \left|x_{i}\right| \leq h
\end{array}\right\} \text { - the semi-implicit method }
$$


where $h>0$ is the sampling period, $i=0,1, \ldots$ The obtained discretized model is fixed-time stable, i.e., its trajectories converge to zero in $N\left(x_{0}\right)$ samplings steps, and $N\left(x_{0}\right)$ is globally bounded. Since $N_{1}\left(x_{0}\right)$ steps $\left(N_{1} \leq 1 / h\right)$ are needed to guarantee $|x(t h)|=\left|x_{i}\right| \leq 1$ for all $i \geq N_{1}$ provided that $\left|x_{0}\right|>1$, and $N_{2}\left(x_{0}\right)$ steps $\left(N_{2} \leq 1 / h\right)$ are needed to reach the origin if $\left|x_{0}\right| \leq 1$, then $N \leq N_{1}+N_{2}$ and

$$
N\left(x_{0}\right) \leq \frac{2}{h} .
$$

Notice that if the control law is implemented in a sampled way as

$$
u(t)=u_{i}, \quad t \in[i h,(i+1) h),
$$

then

$$
x(t)=x_{i}+(t-h) u_{i} \quad t \in[i h,(i+1) h) .
$$

In order to guarantee $x((i+1) h)=x_{i+1}$ the control values $u_{i}$ should be selected as follows

$$
u_{i}=\left\{\begin{array}{ccc}
-\frac{\left|x_{i}\right| x_{i}}{1+h \mid x_{i}} & \text { if } & \left|x_{i}\right| \geq 1, \\
-\operatorname{sign}\left(x_{i}\right) & \text { if } & h<\left|x_{i}\right|<1, \\
-\frac{x_{i}}{h} & \text { if } & \left|x_{i}\right| \leq h,
\end{array}\right\} \text { - the semi-implicit method }
$$

which correspond to the semi-implicit $u_{i}=-\left|x_{i}\right| x_{i+1}$ and the implicit $u_{i} \in-\operatorname{sign}\left(x_{i+1}\right)$ discretizations of the control law, respectively. Therefore, the closed-loop system with the sampled control is also fixed-time stable, i.e., $x(t)=0$ for $t \geq h N\left(x_{0}\right)$. This perfectly corresponds to the continuous-time case. Since $h N\left(x_{0}\right) \rightarrow T\left(x_{0}\right)$ as $h \rightarrow 0^{+}$, we conclude that the same settling time estimate $h N\left(x_{0}\right) \leq T_{\max }=2$ holds even for the system with the sampled control actions.

An example of the consistent discretization in $\mathbb{R}^{n}$ is considered in the Section 6.2.

\section{Discussion}

A big advantage of the consistent discretization algorithms is that the obtained discrete-time system with a constant sampling step inherits the convergence rates of the original continuous-time dynamics (e.g., 
the origin can be reached in a finite number of steps globally in the case with discretization of an FxTS system). This framework can also be used for sampling-time implementation of the controllers providing accelerated convergence to continuous-time plants. The drawback of this method is its computational complexity, since it requires derivation of homogeneous norms on each step, and the consistent discretization always includes implicit methodology, which implies that a nonlinear vector equation has to be solved to calculate $x_{i+1}$.

\subsubsection{Variable-step methods}

Following (Efimov et al., 2019), a state-dependent scaling of the time discretization step is considered in this subsection for explicit and implicit Euler discretization schemes. It is shown that such an approach allows the finite- or fixed-time rates of convergence to be recovered by the discrete-time approximations of solutions, but for an infinite number of steps (in a finite number of steps the convergence to a vicinity of the origin is obtained). It is also demonstrated that relative discretization errors are globally bounded and by decreasing the initial discretization step it is possible to make them arbitrary small, hence, the proposed modification of the Euler method can be indeed used for calculation of solutions of homogeneous dynamics. Comparing to other methods discussed above, utilization of state-dependent discretization steps provides a good compromise between low computational complexity of this approach and good approximation characteristics.

\section{Euler schemes}

As before, consider again a nonlinear system (6.1), where $f: \mathbb{R}^{n} \rightarrow \mathbb{R}^{n}$ ensures forward existence and uniqueness of the system solutions at least locally, $f(0)=0$. For an initial condition $x_{0} \in \mathbb{R}^{n}$ define the corresponding solution by $X\left(t, x_{0}\right)$ for any $t \geq 0$ for which the solution exists. If $f$ is discontinuous, then the solutions are understood in the Filippov's sense (Filippov, 1988).

Let

$$
\sup _{\xi \in S}\|f(\xi)\|<+\infty
$$


and Assumption 6.1 be satisfied.

To introduce modified Euler schemes with state-dependent step, select a basic discretization step $h>0$, define a sequence of time instants $t_{i}$ for $i=0,1, \ldots$ such that $t_{0}=0$ and $t_{i+1}-t_{i}>0$, and denote by $x_{i}$ an approximation of the solution $X\left(t_{i}, x_{0}\right)$ at the corresponding time instant (i.e. $x_{i} \simeq X\left(t_{i}, x_{0}\right)$ and $x_{0}=X\left(0, x_{0}\right)$ ), then the approximation $x_{i+1}$ calculated in accordance with the explicit Euler method is given by:

$$
\begin{aligned}
x_{i+1} & =x_{i}+\frac{h}{\left\|x_{i}\right\|_{\mathbf{d}}^{\nu}} f\left(x_{i}\right), \\
t_{i+1} & =t_{i}+\frac{h}{\left\|x_{i}\right\|_{\mathbf{d}}^{\nu}}
\end{aligned}
$$

for $i=0,1, \ldots$, while the approximation calculated by the implicit Euler method comes from:

$$
\begin{aligned}
x_{i+1} & =x_{i}+\frac{h}{\left\|x_{i+1}\right\|_{\mathbf{d}}^{\nu}} f\left(x_{i+1}\right), \\
t_{i+1} & =t_{i}+\frac{h}{\left\|x_{i+1}\right\|_{\mathbf{d}}^{\nu}}
\end{aligned}
$$

for $i=0,1, \ldots$ In the algorithms (6.24) and (6.25) it is assumed that $x_{i} \neq 0$ or $x_{i+1} \neq 0$ since in the opposite case the discretization stops at the equilibrium due to $f(0)=0$.

Remark 6.1. In order to clarify the relations of these schemes with the ones given previously, and also to motivate the selected design, let us consider a function

$$
\psi_{x_{0}}(t)=\int_{0}^{t}\left\|X\left(s, x_{0}\right)\right\|_{\mathbf{d}}^{\nu} d s
$$

for any $x_{0} \in \mathbb{R}^{n}$, which is well-defined and invertible when the trajectory stays out of the origin, i.e., for all $t \in\left[0, T_{0}\left(x_{0}\right)\right)$ where $T_{0}: \mathbb{R}^{n} \rightarrow \mathbb{R}_{+} \cup\{+\infty\}$ is possibly infinite time of convergence to the origin. Denote $Y\left(\tau, x_{0}\right)=X\left(\psi_{x_{0}}^{-1}(\tau), x_{0}\right)$ for $\tau \in\left[0, \psi_{x_{0}}\left(T_{0}\left(x_{0}\right)\right)\right)$, 
then

$$
\begin{gathered}
\frac{d}{d \tau} Y\left(\tau, x_{0}\right)=\left.\frac{d}{d s} X\left(s, x_{0}\right)\right|_{s=\psi_{x_{0}}^{-1}(\tau)} \frac{1}{\left\|X\left(\psi_{x_{0}}^{-1}(\tau), x_{0}\right)\right\|_{\mathbf{d}}^{\nu}} \\
=\frac{f\left(X\left(\psi_{x_{0}}^{-1}(\tau), x_{0}\right)\right)}{\left\|X\left(\psi_{x_{0}}^{-1}(\tau), x_{0}\right)\right\|_{\mathbf{d}}^{\nu}}=\frac{f\left(Y\left(\tau, x_{0}\right)\right)}{\left\|Y\left(\tau, x_{0}\right)\right\|_{\mathbf{d}}^{\nu}} .
\end{gathered}
$$

Obviously, for any $x_{0} \in \mathbb{R}^{n}$ the asymptotic stability properties of the corresponding solutions $X\left(t, x_{0}\right)$ of the system (6.1) for $t \in\left[0, T_{0}\left(x_{0}\right)\right)$ and $Y\left(\tau, x_{0}\right)$ of $(6.26)$ for $\tau \in\left[0, \psi_{x_{0}}\left(T_{0}\left(x_{0}\right)\right)\right)$ are interrelated, and the system (6.26) is $\mathbf{d}$-homogeneous with degree 0 :

$$
\frac{f(\mathbf{d}(s) y)}{\|\mathbf{d}(s) y\|_{\mathbf{d}}^{\nu}}=\mathbf{d}(s) \frac{f(y)}{\|y\|_{\mathbf{d}}^{\nu}}
$$

for any $y \in \mathbb{R}^{n}$ and $s \in \mathbb{R}$. As it has been discussed in the first part of this section (Efimov et al., 2017), for the case $\nu=0$ and a properly established discretization step $h>0$ the explicit and implicit Euler methods provide an admissible approximation of the solution $y_{i} \simeq Y\left(\tau_{i}, x_{0}\right)$ with $\tau_{i}=i h$ for $i=0,1, \ldots$ :

$$
\begin{aligned}
y_{i+1} & =y_{i}+\frac{h}{\left\|y_{i}\right\|_{\mathbf{d}}^{\nu}} f\left(y_{i}\right), \\
y_{i+1} & =y_{i}+\frac{h}{\left\|y_{i+1}\right\|_{\mathbf{d}}^{\nu}} f\left(y_{i+1}\right) .
\end{aligned}
$$

Hence,

$$
\tau_{i+1}-\tau_{i}=h=\int_{t_{i}}^{t_{i+1}}\left\|X\left(s, x_{0}\right)\right\|_{\mathbf{d}}^{\nu} d s
$$

where $t_{i}$ are the corresponding instants of discretization in the original time $t$, and under an assumption that $\left\|X\left(s, x_{0}\right)\right\|_{\mathbf{d}}^{\nu} \simeq$ const for $s \in\left[t_{i}, t_{i+1}\right]$, i.e., the discretization interval is sufficiently small relative to the velocity of the system, we obtain $t_{i+1}-t_{i} \simeq \frac{h}{\left\|X\left(t_{i}, x_{0}\right)\right\|_{\mathbf{d}}^{\nu}}$ or $t_{i+1}-t_{i} \simeq \frac{h}{\left\|X\left(t_{i+1}, x_{0}\right)\right\|_{\mathbf{d}}^{\nu}}$ for (6.24) and (6.25), respectively. Above such a scheme is used directly for the system (6.1) to approximate the solution $X\left(t_{i}, x_{0}\right)$, which is a significant difference from the 
conventional Euler method. This intuition also highlights the motivation to reduce an Euler computation scheme to a system of degree zero.

One of the main features of (6.24) and (6.25), that is a consequence of this homogeneity property, is as follows:

Proposition 6.4. Let the system (6.1) be $\mathbf{d}$-homogeneous with a degree $\nu \in \mathbb{R}$. If $\left\{x_{i}\right\}_{i=0}^{\infty}$ is a sequence generated by (6.24) or (6.25) for the time instants $\left\{t_{i}\right\}_{i=0}^{\infty}$ with the step $h$ and the initial state $x_{0} \in \mathbb{R}$, then for any $s \in \mathbb{R}, y_{i}=\mathbf{d}(s) x_{i}$ is a sequence obtained by (6.24) or (6.25), respectively, for the instants $e^{-\nu s}\left\{t_{i}\right\}_{i=0}^{\infty}$ with the step $h$ and the initial state $y_{0}=\mathbf{d}(s) x_{0}$.

Note that $y_{i}$ is an approximation of $X\left(e^{-\nu s} t_{i}, y_{0}\right)$ for scaled instants of time.

Corollary 6.12. Let the system (6.1) be $\mathbf{d}$-homogeneous with a degree $\nu \in \mathbb{R}$, and for all $x_{0} \in S$ there exist sequences $\left\{x_{i}\right\}_{i=0}^{\infty}$ obtained by (6.24) or (6.25) with the step $h>0$ possessing one of the following properties:

$$
\begin{gathered}
\sup _{i \geq 0}\left\|x_{i}\right\|<+\infty ; \\
\lim _{i \rightarrow+\infty} x_{i}=0 .
\end{gathered}
$$

Then for any $y_{0} \in \mathbb{R}^{n}$ there exist sequences $\left\{y_{i}\right\}_{i=0}^{\infty}$ generated by (6.24) or (6.25) with the step $h$ and the initial state $y_{0}$ possessing the same property.

In the sequel, the problem of global convergence to zero of the approximations $\left\{x_{i}\right\}_{i=0}^{\infty}$ derived in (6.24) and (6.25) is studied for system in (6.1) satisfying Assumption 6.1 with $\nu \neq 0$ (the case $\nu=0$, or without scaling of the discretization step, has been analyzed in the first part of this section (Efimov et al., 2017) since it is reduced to the conventional Euler schemes), i.e., we will look for conditions providing the properties (6.27) and (6.28) assumed in Corollary 6.12. 


\section{Convergence of sequences $\left\{x_{i}\right\}_{i=0}^{\infty}$ generated by Euler methods}

Existence of some $x_{i+1} \in \mathbb{R}^{n}$ for any $x_{i} \in \mathbb{R}^{n}$ in the explicit case (6.24) is straightforward, but it is not the case of (6.25). According to the result of Corollary 6.12, it is enough to find the conditions of existence of $x_{i+1}$ for all $x_{i} \in S$ in (6.25). Note that in a general case, it is difficult to provide simple conditions for existence and uniqueness of $x_{i+1}$ in the equation (6.25) for any $x_{0} \in S$, but as before, the homogeneity may further simplify the solution under additional mild restrictions on $f$.

Proposition 6.5. Let $f$ be $\mathbf{d}$-homogeneous of degree $\nu \in \mathbb{R}$ and continuous on $S$. Then there is $h_{0}>0$ such that for all $h \in\left(0, h_{0}\right)$ the equation (6.25) for any $x_{i} \in \mathbb{R}^{n}$ has a solution $x_{i+1} \in \mathbb{R}^{n}$.

Thus, for the proposed implicit Euler method with state-dependent discretization step (6.25), the global existence of solutions may be guaranteed by selecting $h$ sufficiently small provided that $f$ is homogeneous and continuous. Then the principal statement of this subsection is as follows:

Theorem 6.13. Let Assumption 6.1 be satisfied, then there exists $h_{0}>0$ such that for any discretization step $h \in\left(0, h_{0}\right]$ the sequences $\left\{x_{i}\right\}_{i=0}^{\infty}$ obtained by (6.24) or (6.25) for any initial state $x_{0} \in \mathbb{R}^{n}$ and the step $h$ possess the following properties:

(a) $\sup _{i=0,1, \ldots}\left\|x_{i}\right\|_{r}<\gamma\left\|x_{0}\right\|_{r}$ for some $\gamma \in(0,+\infty)$;

(b) $\lim _{i \rightarrow+\infty}\left\|x_{i}\right\|_{r}=0$;

(c) for $\nu=0$ the sequence $\left\{x_{i}\right\}_{i=0}^{\infty}$ has an exponential convergence rate; for $\nu<0$ for any $x_{0} \in \mathbb{R}^{n}$ the time of convergence to the origin $t_{+\infty}^{x_{0}}=\lim _{i \rightarrow+\infty} t_{i}$ is finite; and for $\nu>0$ the time of convergence from any initial conditions $x_{0} \in \mathbb{R}^{n}$ to $B(\rho)$ with any $\rho>0$ is also finite independently of $x_{0}$.

If the matrix $\frac{\partial^{2} V(\xi)}{\partial \xi^{2}} \geq 0$ for all $\xi \in \mathbb{R}^{n}$, where $V: \mathbb{R}^{n} \rightarrow \mathbb{R}_{+}$is a $\mathbf{d}$-homogeneous Lyapunov function for (6.1), then $h_{0}>0$ can be selected arbitrarily for (6.25).

The requirement on nonnegative definiteness of the second derivative of 
$V$ is related with the condition of convexity level set of $V$ imposed also in the first part of this section (Efimov et al., 2017).

\section{Absolute and Relative Errors}

Denote $\Xi_{h}\left(x_{0}\right)=X\left(h\left\|x_{0}\right\|_{r}^{-\nu}, x_{0}\right)$ as the value of the solution of (6.1) with the initial condition $x_{0} \in R^{n}$ evaluated in (6.24) or (6.25) after one iteration with the discretization step $h>0$ (at $t_{1}=h\left\|x_{0}\right\|_{r}^{-\nu}$ with $\left.t_{0}=0\right)$. Denote by $\hat{\Xi}_{h}\left(x_{0}\right)$ the estimated value derived by (6.24) or (6.25) for the same $x_{0}$ and $h>0$ (note that $\hat{\Xi}_{h}\left(x_{0}\right)=x_{0}+\frac{h}{\left\|x_{0}\right\|_{r}^{\nu}} f\left(x_{0}\right)$ in the case of (6.24)), then recall the definitions of the approximation errors in this case:

- the absolute error is the magnitude of the difference between the exact value and its approximation:

$$
\Delta^{h}(x)=\left\|\Xi_{h}(x)-\hat{\Xi}_{h}(x)\right\|_{\mathbf{d}}
$$

- the relative error expresses how large the absolute error is compared with the exact value:

$$
\delta^{h}(x)=\frac{\Delta^{h}(x)}{\left\|\Xi_{h}(x)\right\|_{\mathbf{d}}} .
$$

These error functions admit the following useful properties:

Theorem 6.14. Let the system (6.1) be $\mathbf{d}$-homogeneous of degree $\nu \in \mathbb{R}$ and $\hat{\Xi}_{h}(x)$ be calculated by the explicit (6.24) or implicit (6.25) Euler scheme for $x \in \mathbb{R}^{n}$ and $h>0$. Then the functions $\Delta^{h}(x)$ and $\delta^{h}(x)$ are $\mathbf{d}$-homogeneous of degree 1 and 0 , respectively.

Any homogeneous function of degree 0 is globally bounded (it may be discontinuous) if its maximal amplitude is finite being evaluated on $S$. Therefore, if for any initial conditions $x \in S$ the error $\delta^{h}(x)$ stays sufficiently small for a reasonable selection of $h$ (i.e., the one step error of usual Euler discretization approaches is small on the sphere), then the explicit (6.24) and the implicit (6.25) Euler schemes provide a uniformly bounded relative error $\delta^{h}$ globally. Boundedness of $\delta^{h}$ implies that the difference between $\Xi_{h}(x)$ and $\hat{\Xi}_{h}(x)$ stays of the order 
$\Xi_{h}(x)$ (roughly speaking proportional to $x$ ). Indeed, assume that $f$ is continuously differentiable on $S$. In this scenario, the second derivative of the solution $X\left(t, x_{0}\right)$ exists and continuous, and for all $x_{0} \in S$ :

$$
\begin{gathered}
\Xi_{h}\left(x_{0}\right)=X\left(h\left\|x_{0}\right\|_{\mathbf{d}}^{-\nu}, x_{0}\right)=x_{0}+h \dot{X}\left(0, x_{0}\right)+\frac{h^{2}}{2} \ddot{X}\left(\theta, x_{0}\right) \\
=x_{0}+h f\left(x_{0}\right)+\frac{h^{2}}{2} \ddot{X}\left(\theta, x_{0}\right),
\end{gathered}
$$

where $\theta \in[0, h]$, and the Lagrange reminder of Taylor series expansion was used. Then for $x_{0} \in S$ and the explicit discretization algorithm (6.24):

$$
\begin{gathered}
\Delta^{h}\left(x_{0}\right)=\left\|\Xi_{h}\left(x_{0}\right)-\hat{\Xi}_{h}\left(x_{0}\right)\right\|_{\mathbf{d}} \\
=\left\|x_{0}+h f\left(x_{0}\right)+\frac{h^{2}}{2} \ddot{X}\left(\theta, x_{0}\right)-x_{0}-h f\left(x_{0}\right)\right\|_{\mathbf{d}} \\
=\frac{h^{2}}{2}\left\|\ddot{X}\left(\theta, x_{0}\right)\right\|_{\mathbf{d}}
\end{gathered}
$$

for $h \leq 1$ and $\lambda_{\max }\left(G_{\mathbf{d}}\right) \leq 1$ (always can be assumed without loosing generality). By differentiability of $f$, there exists a constant $\varsigma>0$ such that

$$
\sup _{\theta \in[0,1], x_{0} \in S}\left\|\ddot{X}\left(\theta, x_{0}\right)\right\|_{\mathbf{d}} \leq \varsigma,
$$

hence,

$$
\Delta^{h}\left(x_{0}\right) \leq \frac{\varsigma}{2} h^{2}
$$

for all $x_{0} \in S$ and $h \in(0,1]$. The quadratic in $h$ convergence of the absolute error $\Delta^{h}\left(x_{0}\right)$ (the same can be shown for the relative error $\left.\delta^{h}\left(x_{0}\right)\right)$ implies approaching of the real solution $X\left(h, x_{0}\right)$ by its approximation given in (6.24) (Dahlquist and Björck, 2008). This is an interesting and important observation motivating the use of (6.24) and (6.25) in the applications.

Example 6.7. Consider a scalar stable nonlinear system

$$
\dot{x}=-|x| x,
$$


which is homogeneous of degree $\nu=1$ for $\mathbf{d}(s)=e^{s}$, with its corresponding discretizations (6.24) and (6.25):

$$
\begin{gathered}
x_{i+1}=(1-h) x_{i}, t_{i+1}=t_{i}+\frac{h}{\left|x_{i}\right|} ; \\
x_{i+1}=\frac{x_{i}}{1+h}, t_{i+1}=t_{i}+\frac{h}{\left|x_{i+1}\right|},
\end{gathered}
$$

which have a similar form and properties for this case. Both schemes are converging, (6.24) for $h \in(0,1)$ and $(6.25)$ for any $h>0$ (Theorem 6.13 , and the function $V(x)=x^{2}$ can be used with positive second derivative).

Example 6.8. Consider another scalar stable nonlinear system

$$
\dot{x}=-|x|^{0.5} \operatorname{sign}(x),
$$

which is homogeneous of degree $\nu=-0.5$ for $\mathbf{d}(s)=e^{s}$, with its corresponding discretizations (6.24) and (6.25):

$$
\begin{aligned}
& x_{i+1}=(1-h) x_{i}, t_{i+1}=t_{i}+\sqrt{\left|x_{i}\right|} h \\
& x_{i+1}=\frac{x_{i}}{1+h}, t_{i+1}=t_{i}+\sqrt{\left|x_{i+1}\right|} h,
\end{aligned}
$$

where the update law for $x_{i}$ has the same form as in the previous example, and only the time instants $t_{i}$ are scheduled differently (it is not a surprising observation, since the discrete-time dynamics (6.24) and (6.25) are homogeneous of zero degree, then for scalar homogeneous systems (6.1) they have to produce similar expressions). The same convergence properties follow.

Remark 6.2. Note that the time step in (6.24) and (6.25) is stateand degree-dependent. In particular, if $\left\|x_{i}\right\|_{\mathbf{d}} \gg 1$ (it is sufficiently big) and $\nu<0$, then $t_{i+1}-t_{i}=\left\|x_{i}\right\|_{\mathbf{d}}^{-\nu} h \gg h$ in (6.24) and the time step can be too large, which is also related with the obtained accuracy estimates in Theorem 6.14. It is worth stressing that the convergence of these algorithms is not influenced, and this observation deals only with the sampling of discretization. Therefore, for big amplitudes of $x_{i}$ in the case of $\nu<0$ it is admissible to 
use the conventional Euler methods (without a scaling of the time discretization step), which may provide a reasonable accuracy of approximation of the solutions under a more regular sampling (Efimov et al., 2017; Levant et al., 2016).

\section{Robustness with respect to external inputs}

Let us consider a version of the system (6.1) extended by external inputs:

$$
\dot{x}(t)=F(x(t), u(t)), t \geq 0,
$$

with $F: \mathbb{R}^{n+m} \rightarrow \mathbb{R}^{n}$ and $F(x, 0)=f(x)$, where $u: \mathbb{R}_{+} \rightarrow \mathbb{R}^{m}$ is a measurable and essentially bounded function of time. For $x_{0} \in \mathbb{R}^{n}$ and an input $u: \mathbb{R}_{+} \rightarrow \mathbb{R}^{m}$ denote a corresponding solution of the system (6.29) as $X\left(t, x_{0}, u\right)$. The following hypothesis will be imposed on (6.29):

Assumption 6.2. There exist monotone dilations $\mathbf{d}$, $\tilde{\mathbf{d}}$ and $\nu \in \mathbb{R}$ such that

$$
F(\mathbf{d}(s) x, \tilde{\mathbf{d}}(s) u)=e^{\nu s} \mathbf{d}(s) F(x, u)
$$

for all $x \in \mathbb{R}^{n}, u \in \mathbb{R}^{m}$ and $s \in \mathbb{R}$, and there exists $\sigma \in \mathcal{K}_{\infty}$ such that

$$
\sup _{y \in S}\|F(y, u)-F(y, 0)\| \leq \sigma\left(\|u\|_{\tilde{\mathbf{d}}}\right)
$$

for all $u \in \mathbb{R}^{m}$.

If Assumption 6.1 is also satisfied, then since $F(x, 0)=f(x)$, the degrees $\nu$ in these assumptions coincide. Moreover, if assumptions 6.1 and 6.2 are both verified, then the system (6.29) possesses the input-tostate stability (ISS) property with respect to u (Bernuau et al., 2013), and the zone of asymptotic convergence (the asymptotic gain) can be evaluated as

$$
\|x\|_{\mathbf{d}, \infty} \leq \mu\|u\|_{\tilde{\mathbf{d}}, \infty}
$$

where the gain $\mu>0$ and

$$
\|u\|_{\tilde{\mathbf{d}}, \infty}=\operatorname{ess} \sup _{t \geq 0}\|u(t)\|_{\tilde{\mathbf{d}}} .
$$

Let us show that (6.24) and (6.25) preserve the same stability performance for (6.29). Note that being applied to (6.29) these algorithms 
can be formulated as follows:

$$
\begin{aligned}
x_{i+1} & =x_{i}+\frac{h}{\left\|x_{i}\right\|_{\mathbf{d}}^{\nu}} F\left(x_{i}, u_{i}\right), \\
t_{i+1} & =t_{i}+\frac{h}{\left\|x_{i}\right\|_{\mathbf{d}}^{\nu}}
\end{aligned}
$$

or

$$
\begin{aligned}
x_{i+1} & =x_{i}+\frac{h}{\left\|x_{i+1}\right\|_{\mathbf{d}}^{\nu}} F\left(x_{i+1}, u_{i+1}\right), \\
t_{i+1} & =t_{i}+\frac{h}{\left\|x_{i+1}\right\|_{\mathbf{d}}^{\nu}}
\end{aligned}
$$

for $i=0,1, \ldots$, respectively, where $x_{i}$ is an estimate of $X\left(t_{i}, x_{0}, u\right)$ as before and $u_{i}=u\left(t_{i}\right)$.

Theorem 6.15. Let assumptions 6.1 and 6.2 be satisfied, then there exists $h_{0}>0$ such that for any discretization step $h \in\left(0, h_{0}\right]$ the discrete-time systems (6.30) or (6.31) are ISS (see (Jiang and Wang, 2001) for the definition of this property and also for its equivalent Lyapunov characterizations for discrete-time systems), and for any initial state $x_{0} \in \mathbb{R}^{n}$ and any bounded input $u: \mathbb{R}_{+} \rightarrow \mathbb{R}^{m}$ the corresponding sequences $\left\{x_{i}\right\}_{i=0}^{\infty}$ enter in the set where

$$
\sup _{i \geq 0}\left\|x_{i}\right\|_{\mathbf{d}} \leq \mu \sup _{i \geq 0}\left\|u_{i}\right\|_{\tilde{\mathbf{d}}}
$$

Therefore, the proposed schemes (6.24) and (6.25) (or (6.30) and (6.31)) for approximation of solutions of a homogeneous system (6.29) keep unchanged after discretization the convergence rates and stability margins in the presence of perturbations.

Remark 6.3. Note that for an input $u(t) \neq 0$ the corresponding trajectory may visit the origin, but does not stay there due to disturbance presence. In such a case the algorithms (6.30) and (6.31) will decrease to zero the amplitude of the time step for the systems with negative degree or augment it till infinity for the systems with positive degree following their construction. In order 
to avoid this issue, the upper and lower limits $0<\underline{h}<\bar{h}<+\infty$ on $h\left\|x_{i}\right\|_{\mathbf{d}}^{-\nu}$ or $h\left\|x_{i+1}\right\|_{\mathbf{d}}^{-\nu}$ must be imposed in (6.30) and (6.31), respectively.

\section{Discussion}

For a suffisiently small value of $h$, the Euler methods (6.24) and (6.25) provide a good approximation of the system solutions (Theorem 6.14). For the implicit Euler scheme, under a mild constraint, it has been proven that solutions always exist and converge globally to zero for any $h$ if the Hessian of the Lyapunov function of the system is nonnegative definite (Theorem 6.13). Both approaches, (6.24) and (6.25) ((6.30) and (6.31)), preserve the input-to-state stability of the homogeneous dynamics (6.29) with the asymptotic gain of the continuous-time counterpart (Theorem 6.15). The drawback of these approaches is that finite/fixed-time convergence is recovered in infinite number of steps.

\subsubsection{Conclusion and comparison of given discretization tools}

For Euler algorithms (with constant (6.2), (6.3) or state-dependent (6.24), (6.25) steps), homogeneity simplifies analysis of properties of the obtained discrete approximations of solutions. For example, a certain scalability between approximations calculated for different initial conditions and discretization steps is established in propositions 6.3 or 6.4 .

Application of the conventional explicit Euler method (6.2) leads to local approximations of solutions of homogeneous systems with nonzero degrees (having finite-time or fixed-time rates of convergence). To calculate global approximations, the implicit method (6.3) can be used, but still asymptotic convergence to the origin is guaranteed in infinite number of steps.

Introducing the state-dependent step in (6.24), (6.25), the obtained approximations can be made globally converging for asymptotically stable homogeneous systems provided that $h$ is selected sufficiently small. In addition, the finite/fixed-time rates of convergence can be guaranteed in infinite number of iterations (Theorem 6.13). In comparison with the 


\begin{tabular}{|c|c|c|c|c|c|}
\hline & $(6.2)$ & $(6.3)$ & $(6.24)$ & $(6.25)$ & Consistent \\
\hline \hline Domain & Local & Global & Global & Global & Global \\
\hline Rates & Asymptotic & Asymptotic & Finite/Fixed & Finite/Fixed & Finite/Fixed \\
\hline Number of steps & Infinite & Infinite & Infinite & Infinite & Finite \\
\hline Complexity & + & ++ & + & ++ & +++ \\
\hline
\end{tabular}

Table 6.1: Comparison of different discretization approaches

standard implicit Euler method (6.3) (having a similar performance), the advantages are that the computationally simpler explicit method (6.24) can be used, and that due to time scaling the approximations have indeed accelerated convergence rates.

The consistent discretization approach gets its name since it provides the finite/fixed-time convergence rates in discrete-time for the solution approximations, and it can also be used for control implementation. It is also the most demanding approach in computations.

Summarizing this discussion, the mentioned properties are briefly described in Table 6.1.

\subsection{Digital Implementation of ILF-based algorithms}

\subsubsection{Practical realization in the form of a linear switched feedback}

As discussed in Section 4.2, in order to implement an implicit homogeneous control in practice, a numerical algorithm for a computation of the canonical homogeneous norm is required. This norm can be computed explicitly for $n \leq 2$ approximated by an explicit homogeneous norm for $n \geq 3$. However, even for the second order case the analytical representation of the canonical homogeneous norm is rather cumbersome, so a digital realization of the homogeneous control law requires more computational power than the linear algorithm. Therefore, a sufficiently simple computational algorithm is required for its successful digital realization. Some additional properties of the implicit homogeneous controller are established below for this purpose.

Recall that a non-empty compact set $\Omega \subset \mathbb{R}^{n}$ is said to be a strictly positively invariant for a dynamical system if $x\left(t_{0}\right) \in \Omega \Rightarrow x(t) \in$ int $\Omega, t \geq t_{0}$, where $x$ denotes a trajectory of the dynamical system and int $\Omega$ denotes the interior of $\Omega$. 
Theorem 6.16 (Polyakov, 2020). If all conditions of Corollary 4.6 hold then for any fixed $r>0$ the closed $\mathbf{d}$-homogeneous ball $\bar{B}_{\mathbf{d}}(r)$ is a strictly positively invariant compact set of the closed-loop system (4.25) with the linear control

$$
u_{r}(x)=K_{0} x+r^{1+\mu} K \mathbf{d}(-\ln r) x .
$$

Now we assume that the value $\|x(t)\|_{\mathbf{d}}$ in the implicit homogeneous controller can be changed only in some sampled instances of time. Let us show that the corresponding linear switched feedback robustly stabilize the perturbed linear system.

Corollary 6.17 (Polyakov et al., 2016b, Polyakov, 2020). If

1) the conditions of Corollary 4.6 hold;

2) $\left\{t_{i}\right\}_{i=0}^{+\infty}$ is an arbitrary sequence of time instances such that

$$
0=t_{0}<t_{1}<t_{2}<\ldots \quad \text { and } \quad \lim _{i \rightarrow+\infty} t_{i}=+\infty ;
$$

3) the linear switched control $u$ has the form

$$
u(x(t))=K_{0} x(t)+\left\|x\left(t_{i}\right)\right\|_{\mathbf{d}}^{1+\mu} K \mathbf{d}\left(-\ln \left\|x\left(t_{i}\right)\right\|_{\mathbf{d}}\right) x(t), \quad t \in\left[t_{i}, t_{i+1}\right)
$$

then the closed-loop system (4.25), (6.33) is globally asymptotically stable.

The linear switched control (6.33) is obtained from the nonlinear homogeneous one. It can be utilized, for example, in the case when the control system is already equipped with a linear (e.g. analog) controller allowing a change of feedback gains with some sampling period.

According to the proven corollary, the proposed sampled-time realization of the implicit homogeneous controller guarantees asymptotic stabilization of the closed-loop system independently of the dwell time (a time between two sampling instants). Such property is not usual 
for sampled and switched control systems with additive disturbances (Liberzon, 2003). However, without an assumption on the dwell-time we cannot estimate the convergence rate of this system. Obviously, if the dwell time tends to zero the convergence rate tends to the rate of the original continuous system.

To implement the obtained switched linear feedback through these algorithms, an on-line computation of the canonical homogeneous is required. Fortunately, rather simple numerical procedures can be utilized for this purpose.

Let $0=t_{0}<t_{1}<t_{2}<\ldots$ be an arbitrary sequence of time instants, $\lim t_{i}=+\infty$, and $a>0, b>0$ be the tuning parameters.

Algorithm 6.1 (Polyakov et al., 2015a, Polyakov, 2020).

Initialization $\underline{V}=a ; \bar{V}=b ; N_{\max } \in \mathbb{N}$;

Step:

if $x^{\top}\left(t_{i}\right) \mathbf{d}^{\top}(-\ln \bar{V}) P \mathbf{d}(-\ln \bar{V}) x\left(t_{i}\right)>1$ then

$$
\underline{V}=\bar{V} ; \bar{V}=\min (b, 2 \bar{V}) ;
$$

elseif $x^{\top}\left(t_{i}\right) \mathbf{d}^{\top}(-\ln \underline{V}) P \mathbf{d}(-\ln \underline{V}) x\left(t_{i}\right)<1$ then

$$
\bar{V}=\underline{V} ; \underline{V}=\max (0.5 \underline{V}, a) ;
$$

else

$$
\begin{aligned}
& \text { for } i=1: N_{\max } \\
& \quad V=\frac{V+\bar{V}}{2} ; \\
& \quad \text { if } x^{\top}\left(t_{i}\right) \mathbf{d}^{\top}(-\ln \underline{V}) P \mathbf{d}(-\ln \underline{V}) x\left(t_{i}\right)<1 \text { then } \\
& \quad \bar{V}=V ; \\
& \quad \text { else } \underline{V}=V ; \\
& \quad \text { endif; } \\
& \text { endfor; }
\end{aligned}
$$

endif;

$\left\|x\left(t_{i}\right)\right\|_{\mathbf{d}} \approx \bar{V}$

Let $x\left(t_{i}\right) \in \mathbb{R}^{n} \backslash\{\mathbf{0}\}$ be a given vector and $a=0, b=+\infty$. If the Step of the presented algorithm is applied recurrently many times to the same $x\left(t_{i}\right)$ then Algorithm 6.1 guarantees: 
1) a localization of the unique positive root of the equation

$$
\left\|\mathbf{d}(-\ln V) x\left(t_{i}\right)\right\|=0
$$

with respect to $V>0$, i.e. $V \in[\underline{V}, \bar{V}]$;

2) improvement of the obtained localization by means of the bisection method, i.e. $(\bar{V}-\underline{V}) \rightarrow 0$ as number of steps tends to infinity.

Such an application of Algorithm 6.1 allows us to calculate $V \approx$ $\left\|x\left(t_{i}\right)\right\|$ with rather high precision, but it requires a high computational capability of a digital device. If the computational power is very restricted, then the Step of Algorithm 6.1 may be realized just once at each sampled instant of time. The practical stability of the closed-loop system can be guaranteed in this case. Indeed, Theorem 6.16 proves that the d-homogeneous ball $\bar{B}_{\mathbf{d}}(\bar{V})$ is a strictly positively invariant set of the the closed-loop system with the control $\left.u(x)=\bar{V}^{\mu} K \mathbf{d}(-\ln \bar{V})\right)$. If the root of the equation $\left\|\mathbf{d}(-\ln V) x\left(t_{i}\right)\right\|=0$ is localized (i.e. $\left\|x\left(t_{i}\right)\right\|_{\mathbf{d}} \leq \bar{V}$ ), Algorithm 6.1 always selects an upper estimate of $V$ to guarantee $x\left(t_{i}\right) \in \bar{B}_{\mathbf{d}}(\bar{V})$. This means that $\left\|x\left(t_{i}\right)\right\|_{\mathbf{d}}$ never leaves the ball $\bar{B}_{\mathbf{d}}(\bar{V})$ even when $x(t)$ varies in time.

\subsubsection{Consistent discretization of ILF-based algorithms}

Let us consider the linear system with the implicit homogeneous control (4.11) recalled here as follows

$$
\begin{gathered}
\dot{x}=f(x):=A x+B u, \\
u_{\nu}(x):=K_{0} x+\|x\|_{\mathbf{d}}^{1+\nu} K \mathbf{d}\left(-\ln \|x\|_{\mathbf{d}}\right) x,
\end{gathered}
$$

where $x=\left(x_{1}, x_{2}, \ldots, x_{n}\right)^{\top}, A \in \mathbb{R}^{n \times n}$ is a $\mathbf{d}$-homogeneous matrix of a degree $\nu \in\{-1,1\}$, the matrix $B \in \mathbb{R}^{n \times m}$ is such that the pair $\{A, B\}$ is controllable, $K \in \mathbb{R}^{m \times n}$ is the matrix of control gains, $\mathbf{d}$ is a dilation in $\mathbb{R}^{n}$ and $K_{0} \in \mathbb{R}^{m \times n}$ is such that the matrix $A_{0}=A+B K_{0}$ is nilpotent.

The closed-loop system is homogeneous of the degree $\nu$ :

$$
f(\mathbf{d}(s) x)=A \mathbf{d}(s) x+B u_{\nu}(\mathbf{d}(s) x)=e^{\nu s} \mathbf{d}(s)\left(A x+B u_{\nu}(x)\right)=e^{\nu s} \mathbf{d}(s) f(x) .
$$

The equivalent transformed homogeneous system with

$$
y=\|x\|_{\mathbf{d}} \mathbf{d}\left(-\ln \|x\|_{\mathbf{d}}\right) x
$$


has the form

$$
\dot{y}=\|y\|^{1+\nu}\left(\frac{\left(I_{n}-G_{\mathbf{d}}\right) y y^{\top} P}{y^{\top} P G_{\mathbf{d}} y}+I_{n}\right) f\left(\frac{y}{\|y\|}\right),
$$

where

$$
f\left(\frac{y}{\|y\|}\right)=(A+B K) \frac{y}{\|y\|},
$$

and $\|y\|=\sqrt{y^{\top} P y}$ with $P$ satisfying (3.10).

Let the gain vector $K$ and a positive definite matrix $P \succ 0$ be selected as follows:

$$
\left(A_{0}+B K+G_{\mathbf{d}}\right)^{\top} P+P\left(A_{0}+B K+G_{\mathbf{d}}\right)=0, \quad P G_{\mathbf{d}}+G_{\mathbf{d}}^{\top} P \succ 0,
$$

where $G_{\mathbf{d}} \in \mathbb{R}^{n \times n}$ is the generator of the dilation $\mathbf{d}(s)=e^{s G_{\mathbf{d}}}, s \in$ $\mathbb{R}$. Such a selection is always possible (see Polyakov et al., 2016b or Polyakov, 2020). In this case, we derive

$$
\begin{gathered}
\tilde{f}(y):=\left(\frac{\left(I_{n}-G_{\mathbf{d}}\right) y y^{\top} P}{y^{\top} P G_{\mathbf{d}} y}+I_{n}\right)(A+B K) \frac{y}{\|y\|}= \\
\frac{1}{\|y\|} \frac{\left(I_{n}-G_{\mathbf{d}}\right) y y^{\top} P(A+B K) y}{y^{\top} P G_{\mathbf{d}} y}+(A+B K) \frac{y}{\|y\|}= \\
\frac{1}{\|y\|} \frac{\left(I_{n}-G_{\mathbf{d}}\right) y\left(-y^{\top} P G_{\mathbf{d}} y\right)}{y^{\top} P G_{\mathbf{d}} y}+(A+B K) \frac{y}{\|y\|}= \\
\left(A+B K+G_{\mathbf{d}}-I_{n}\right) \frac{y}{\|y\|} .
\end{gathered}
$$

Case $\nu=-1$.

For the homogeneous system with negative degree we apply Theorem 6.9. The consistent discretization (6.20) has the following representation

$$
y_{i} \in y_{i+1}+h\left(I_{n}-\tilde{A}\right) \tilde{F}\left(y_{i+1}\right), \quad h>0, i=0,1,2, \ldots
$$

where $\tilde{A}=A+B K+G_{\mathbf{d}}$ such that $\tilde{A}^{\top} P+P \tilde{A}=0$ and

$$
\tilde{F}(y)=\left\{\begin{array}{ccc}
\left\{\frac{y}{\|y\|}\right\} & \text { if } & y \neq 0 \\
B(1) & \text { if } & y=0
\end{array}\right.
$$

where $B(1)$ is the unit ball in $\mathbb{R}^{n}$ with the norm $\|y\|=\sqrt{y^{\top} P y}$. Notice that the condition (6.36) implies that $I_{n}-\tilde{A}$ is invertible.

Let us denote $q_{i+1}=\left\|y_{i+1}\right\|$ and $z_{i+1}=\frac{y_{i+1}}{\left\|y_{i+1}\right\|}$. Then the inclusion (6.37) has the following solution 
- if $y_{i}^{\top}\left(I_{n}-\tilde{A}\right)^{-\top} P\left(I_{n}-\tilde{A}\right)^{-1} y_{i} \leq h^{2}$ then

$$
q_{i+1}=0 \quad \text { and } \quad z_{i+1}=h^{-1}\left(I_{n}-h \tilde{A}\right)^{-1} y_{i} ;
$$

- otherwise, $q_{i+1}$ and $z_{i+1}$ are derived as the solution to

$$
\left(\left(q_{i+1}+h\right) I_{n}-h \tilde{A}\right) z_{i+1}=y_{i}, \quad z_{i+1}^{\top} P z_{i+1}=1,
$$

where $y_{i}=\left\|x_{i}\right\|_{\mathbf{d}} \mathbf{d}\left(-\ln \left\|x_{i}\right\|_{\mathbf{d}}\right) x_{i}$. Solution to (6.39) always exists due to Theorem 6.9. To find it the equation

$$
y_{i}^{\top}\left(\left(q_{i+1}+h\right) I_{n}-h \tilde{A}\right)^{-\top} P\left(\left(q_{i+1}+h\right) I_{n}-h \tilde{A}\right)^{-1} y_{i}=1,
$$

that is polynomial with respect to $q_{i+1}$, must be initially solved. For $n=2$ the system (6.39) implies a quartic equation with respect to $q_{i+1}$, so it can be solved explicitly using Ferrari formulas. In other cases some proper computational procedure can be utilized. In all numerical experiments we consider the model of the controlled double integrator:

$$
A=\left(\begin{array}{ll}
0 & 1 \\
0 & 0
\end{array}\right), \quad B=\left(\begin{array}{l}
0 \\
1
\end{array}\right) .
$$

The simulation results for $\nu=-1, x_{0}=\left(\begin{array}{ll}0.2247 & 0.4494\end{array}\right)^{\top}$ and

$$
\begin{gathered}
\mathbf{d}(s)=\left(\begin{array}{cc}
e^{2 s} & 0 \\
0 & e^{s}
\end{array}\right), \quad P=\left(\begin{array}{ll}
9.1050 & 1.7829 \\
1.7829 & 0.8914
\end{array}\right), \\
K=\left(\begin{array}{ll}
-10.2139 & -3.0000
\end{array}\right),
\end{gathered}
$$

are given in Fig. 6.1, where the developed discretization scheme is compared with the explicit Euler scheme. The simulations confirm finite-time convergence of $\left\{x_{i}\right\}$ to zero in a finite number of steps for the consistent discrete-time model, where

$$
x_{i}=\mathbf{d}\left(\ln \left\|y_{i}\right\|\right) \frac{y_{i}}{\left\|y_{i}\right\|}
$$

and $\left\{y_{i}\right\}$ is the solution to (6.37), while the system obtained using the explicit Euler discretization is not even asymptotically stable (see Fig. 6.1).

Case $\nu=1$. 

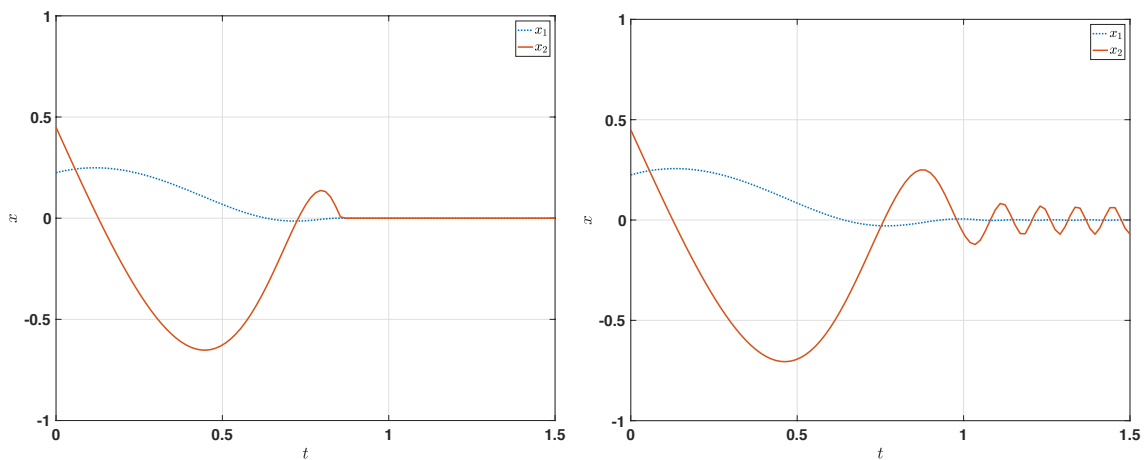

Figure 6.1: Comparison of the discrete-time models obtained by means of the consistent discretization (left) and explicit Euler method (right) for $h=0.015$ and $\nu=-1$.

In this case we use Theorem 6.11. The semi-implicit discretization (6.23) gives

$$
\left(I_{n}+h\left\|y_{i}\right\|\left(I_{n}-\tilde{A}\right)\right) y_{i+1}=y_{i}, \quad i=0,1,2, \ldots
$$

The matrix $\left(I_{n}+h\left\|y_{i}\right\|\left(I_{n}-\tilde{A}\right)\right)$ has only positive eigenvalues for any $h>0$ and any $\left\|y_{i}\right\|$ (since $\tilde{A}^{\top} P+P \tilde{A}=0$ ), then it is invertible, so

$$
y_{i+1}=\left(I_{n}+h\left\|y_{i}\right\|\left(I_{n}-\tilde{A}\right)\right)^{-1} y_{i} .
$$

The results of the numerical simulation for $\nu=1, n=2, x_{0}=$ $\left(\begin{array}{ll}13.255 & 0\end{array}\right)^{\top}$ and

$$
\begin{gathered}
\mathbf{d}(s)=\left(\begin{array}{cc}
e^{s} & 0 \\
0 & e^{2 s}
\end{array}\right), \quad P=\left(\begin{array}{ll}
3.6173 & 2.6173 \\
2.6173 & 2.6173
\end{array}\right), \\
K=\left(\begin{array}{ll}
-1.3821 & -3.0000
\end{array}\right)
\end{gathered}
$$

are presented in Fig. 6.2, where the developed consistent discretization scheme is compared with the explicit Euler scheme.

The simulations show an oscillatory behavior of the discrete-time model obtained using the explicit Euler scheme for $h=0.04$ (see Fig. 6.2 ). For $h>0.05$ the explicit scheme was found to be unstable (solution blows up for the given $x_{0}$ ). 

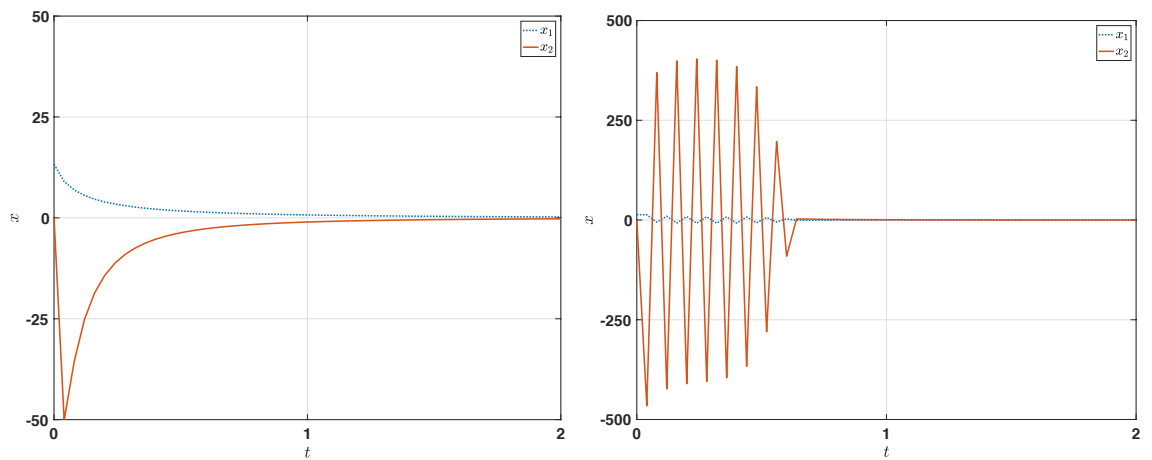

Figure 6.2: Comparison of the discrete-time models obtained by means of the consistent discretization (left) and explicit Euler method (right) for $h=0.04$ and $\nu=1$.

The consistency of the discrete-time model (6.41), (6.40), obtained using the discretization scheme (6.23), is confirmed by numerical experiments. The nearly fixed-time stability is observed in simulations even for large sampling periods $(h=1)$ and large initial conditions $\left(\left\|x_{0}\right\|\right.$ is of the order $\left.10^{20}\right)$.

\section{On digital implementation of a homogeneous control using the con- sistent discretization}

The discretization schemes given above are developed for a numerical simulation of finite-time and fixed-time stable homogeneous ODEs. However, they can also be utilized for a digital (sampled-time) implementation of finite-time or fixed-time controllers. Indeed, let us consider again the example given in the previous section (Case $\nu=-1$ ).

The control (6.35) for $\nu=-1$ is proven to be robust with respect to bounded disturbances (see Corollary 4.6), i.e. the origin of the continuous-time closed-loop system

$$
\dot{x}=A x+B\left(u_{\nu}(x)+\gamma(t, x)\right), \quad t>0
$$

remains globally uniformly finite-time stable provided that $|\gamma|<\gamma_{0}$ and $\gamma_{0}$ is sufficiently small.

Taking into account $x=\mathbf{d}(\ln \|y\|) \frac{y}{\|y\|}$ and $u_{\nu}(\mathbf{d}(s) x)=u_{\nu}(x)$ for $\nu=-1$, we derive that the consistent discretization of the control law 
(6.35) can be defined as follows

$$
u_{\nu}\left(x_{i+1}\right)=u_{\nu}\left(\frac{y_{i+1}}{\left\|y_{i+1}\right\|}\right)=u_{\nu}\left(z_{i+1}\right)=K z_{i+1},
$$

where $z_{i+1}$ is given by (6.38) or (6.39). According to the conventional implicit discretization technique Huber et al., 2016, Acary et al., 2012, Miranda-Villatoro et al., 2017, Miranda-Villatoro et al., 2018 this value is suggested to be selected for the time interval $\left[t_{i}, t_{i+1}\right)$ during a digital implementation of the control law (6.35) in the system (6.34):

$$
u(t)=u_{i}:=K z_{i+1}, \quad t \in[i h,(i+1) h) .
$$

The solution to the perturbed system (6.42) in this case is given by $x(t)=e^{A t} x_{i}+\int_{0}^{t} e^{A(t-s)} B\left(u_{i}+\gamma(s, x(s)) d s, t \in[i h,(i+1) h), x_{i}:=x(i h)\right.$.

Case $\gamma \equiv 0$. The implicit sampled control (6.43) obtained using the consistent discretization completely rejects the numerical chattering (the numerical chattering is unmodeled oscillations in a control system caused by discretization errors of a continuous-time control algorithm, see Huber et al., 2016 for more details and Figure 6.4) in both input and state of the system, while the explicit scheme $u_{i}=u_{\nu}\left(x_{i}\right)$ always generates the chattering (see Figure 6.3). The simulation results for $\gamma=0$ and another sampling periods can be also found in Polyakov et al., 2019.

Case $\gamma \neq 0$. The implicit homogeneous control remains efficient for rejection of the perturbation $\gamma=0.4 \cos (2 t)$ (see Figure 6.5). Noised measurements imply an expectable degradation of the control precision (Figure 6.6). However, the chattering magnitude of the consistently discretized controller in the noised and perturbed case is still less than the chattering magnitude of the explicitly discretized controller in the disturbance-free case.

Notice that the consistently discretized implicit homogeneous control tracks (rejects) the matched perturbation $\gamma=0.4 \cos (2 t)$ since $u \approx-\gamma$ when $x \approx \mathbf{0}$ (see the right figure 6.5). This happens without any knowledge about $\gamma$. Such a behavior is observed only for "slowly-varying" perturbations. To reject a "faster" disturbance the sampling period has 

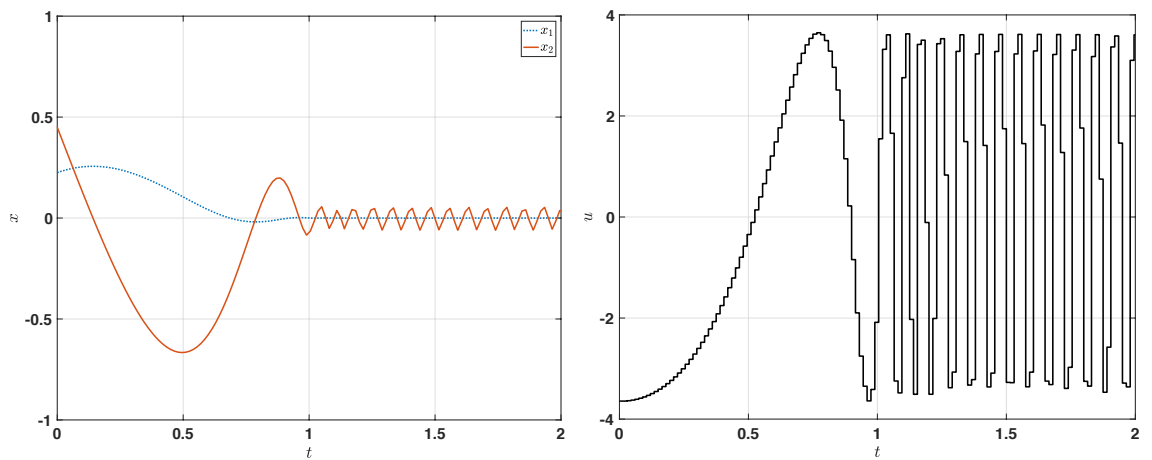

Figure 6.3: Evolution of the system (6.44) with the explicitly discretized control: $u_{i}=u_{\nu}\left(x_{i}\right), h=0.015, \nu=-1$ and $\gamma=0$.
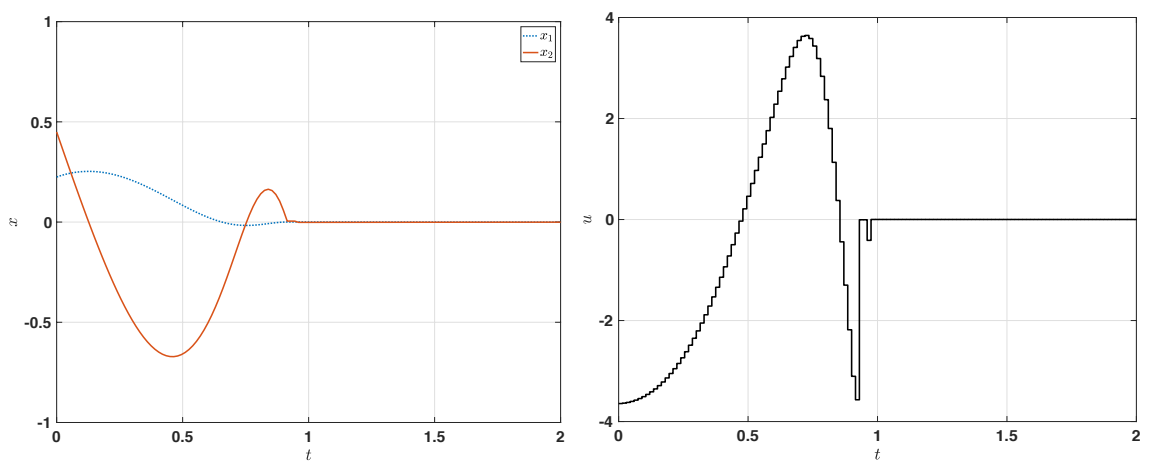

Figure 6.4: Evolution of the system (6.44) with the consistently discretized control: $u_{i}=K z_{i+1}, h=0.015, \nu=-1$ and $\gamma=0$.

to be decreased. In the view of Corollary 4.6, a set of perturbations to be rejected should "tend" to a ball in $L^{\infty}$ as $h \rightarrow 0$. 

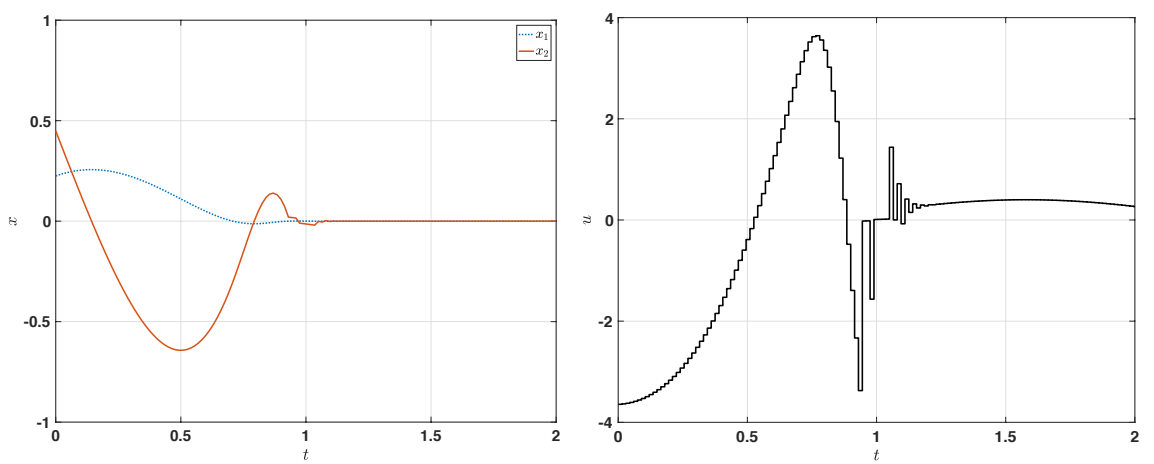

Figure 6.5: Evolution of the system (6.44) with the consistently discretized control $u_{i}=K z_{i+1}, h=0.015$ and $\gamma=0.4 \cos (2 t)$.
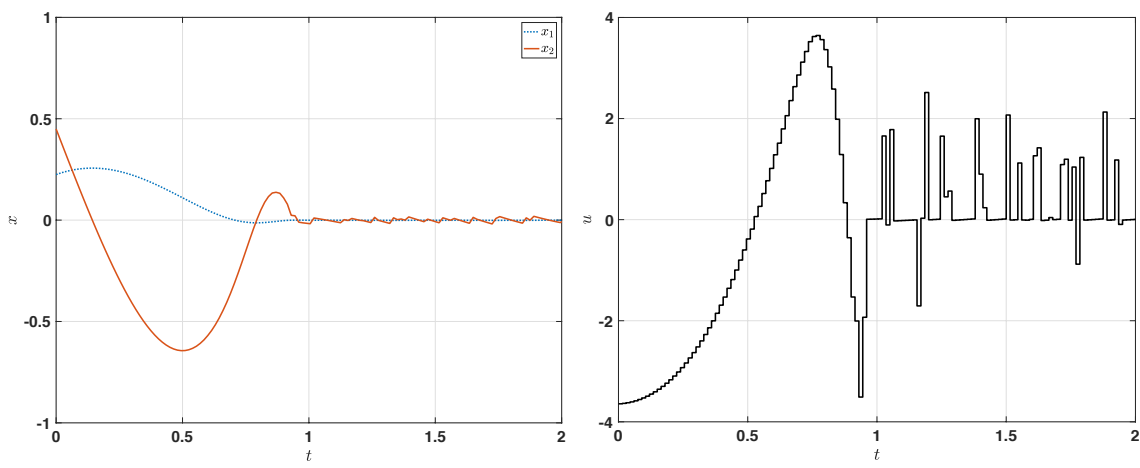

Figure 6.6: Evolution of the system (6.44) with the consistently discretized control $u_{i}=K z_{i+1}, h=0.015, \gamma=0.4 \cos (2 t)$ and with a uniformly distributed measurement noise of the magnitude $10^{-3}$ 


\section{Extensions}

\subsection{Time-delay systems}

The definitions of FTS/FxTS can be extended to time-delay systems with mild modifications. The differences appear in the our methods to verify these stability properties. For ODEs two methods can be applied based on Lyapunov functions and the theory of homogeneous systems.

The homogeneity concept can also be adapted to time-delay systems, either using the fact that any retarded system can be embedded in an evolution equation (Polyakov et al., 2016a) or developing directly the notion of weighted homogeneity to this kind of dynamics (Efimov et al., 2014a; Efimov et al., 2016). In the former case all results previously obtained for ODE systems can be recovered in the time-delay scenario, however, at the price that in such a homogeneous system the delay has to be scaled with the state. The latter approach leads to the solutions admitting a constant delay, but the stability of these homogeneous systems with negative/positive degree does not imply FTS/FxTS (Efimov et al., 2016) (some additional restrictions related with uniformity of convergence have to be introduced).

Returning to Lyapunov's ideas, there exist two generic frameworks assessing stability of time-delay systems, which are based on analysis of 
a Lyapunov-Razumikhin function or a Lyapunov-Krasovskii functional (Kolmanovskii and Myshkis, 1999; Fridman, 2014). The latter method has been proven to be equivalent to the asymptotic stability property for some particular classes of the time-delay systems (Pepe and Karafyllis, 2013; Pepe et al., 2017; Efimov and Fridman, 2020), and it can also be used to establish finite-time stability (Moulay et al., 2008). The former approach is only sufficient for the asymptotic stability (Kolmanovskii and Myshkis, 1999; Fridman, 2014), and it is less intuitive while obtaining the rate of solution convergence (Myshkis, 1995; Aleksandrov and Zhabko, 2012; Efimov et al., 2014b; Efimov and Aleksandrov, 2020). An advantage of Lyapunov-Razumikhin approach with respect to LyapunovKrasovskii one is that in many nonlinear cases it is simpler to find a Lyapunov-Razumikhin function than a Lyapunov-Krasovskii functional (Efimov et al., 2014a; Efimov et al., 2016) (e.g., a Lyapunov function for the delay-free case can be tested).

In this subsection, the definitions of accelerated convergence rates are described for time-delay systems. Next, the necessary and sufficient conditions are formulated using Lyapunov-Krasovskii approach. The Lyapunov-Razumikhin approach is finally recalled.

\subsubsection{Preliminaries}

We denote by $C_{[a, b]}=C\left([a, b], \mathbb{R}^{n}\right),-\infty<a<b<+\infty$ the Banach space of continuous functions $\phi:[a, b] \rightarrow \mathbb{R}^{n}$ with the uniform norm $\|\phi\|=\sup _{a \leq \varsigma \leq b}\|\phi(\varsigma)\|_{\mathbb{R}^{n}}$.

Consider an autonomous functional differential equation of retarded type (Kolmanovsky and Nosov, 1986):

$$
\frac{d x(t)}{d t}=f\left(x_{t}\right), t \geq 0,
$$

where $x(t) \in \mathbb{R}^{n}$ and $x_{t} \in C_{[-\tau, 0]}$ is the state function, $x_{t}(s)=x(t+s)$, $-\tau \leq s \leq 0$ and $\tau>0$ is a finite delay; $f \in C\left(C_{[-\tau, 0]}, \mathbb{R}^{n}\right)$ with $f(\mathbf{0})=0$ and it is such that the solutions in forward time for the system (7.1) exist and are unique (Kolmanovsky and Nosov, 1986). Let $x\left(t, x_{0}\right)$ denote such a unique solution satisfying the initial condition $x\left(s, x_{0}\right)=x_{0}(s)$ for $-\tau \leq s \leq 0$ and $x_{0} \in C_{[-\tau, 0]}$, which is defined on some finite time interval $[-\tau, T)$ with $0<T \leq+\infty$. 
For a locally Lipschitz continuous function $V: \mathbb{R}^{n} \rightarrow \mathbb{R}_{+}$the upper directional Dini derivative is defined as follows:

$$
D^{+} V(x) v=\limsup _{h \rightarrow 0^{+}} \frac{V(x+h v)-V(x)}{h}
$$

for any $x \in \mathbb{R}^{n}$ and $v \in \mathbb{R}^{n}$. The upper right-hand Dini derivative of a continuous functional $V: C_{[-\tau, 0]} \rightarrow \mathbb{R}$ along the system (7.1) solutions is defined as

$$
D^{+} V(\phi)=\limsup _{h \rightarrow 0^{+}} \frac{V\left(\phi_{h}\right)-V(\phi)}{h}
$$

for any $\phi \in C_{[-\tau, 0]}$, where $\phi_{h} \in C_{[-\tau, 0]}$ for $0<h<\tau$ is given by

$$
\phi_{h}= \begin{cases}\phi(\theta+h), & \theta \in[-\tau,-h) \\ \phi(0)+f(\phi)(\theta+h), & \theta \in[-h, 0] .\end{cases}
$$

\subsubsection{Stability definitions}

Let $\Omega$ be a neighborhood of zero in $C_{[-\tau, 0]}$.

Definition 7.1. (Moulay et al., 2008; Kolmanovskii and Myshkis, 1999; Fridman, 2014; Efimov and Aleksandrov, 2020) The origin of the system (7.1) is said to be

(a) stable if there is $\sigma \in \mathcal{K}$ such that for any $x_{0} \in \Omega$, the solutions are defined and $\left\|x\left(t, x_{0}\right)\right\| \leq \sigma\left(\left\|x_{0}\right\|\right)$ for all $t \geq 0$;

(b) asymptotically stable if it is stable and $\lim _{t \rightarrow+\infty}\left\|x\left(t, x_{0}\right)\right\|=0$ for any $x_{0} \in \Omega$;

(c) FTS if it is stable and for any $x_{0} \in \Omega$ there exists $0 \leq$ $T^{x_{0}}<+\infty$ such that $x\left(t, x_{0}\right)=0$ for all $t \geq T^{x_{0}}$. The functional $T\left(x_{0}\right)=\inf \left\{T^{x_{0}} \geq 0: x\left(t, x_{0}\right)=0 \forall t \geq T^{x_{0}}\right\}$ defines the settling time of the system (7.1);

(d) nearly FxTS if it is stable and for any $\varrho>0$ there exists $0<T_{\varrho}<+\infty$ such that $\left\|x\left(t, x_{0}\right)\right\| \leq \varrho$ for all $t \geq T_{\varrho}$ and all $x_{0} \in \Omega$;

(e) FxTS if it is FTS and $\sup _{x_{0} \in \Omega} T\left(x_{0}\right)<+\infty$.

If $\Omega=C_{[-\tau, 0]}$, then the corresponding properties are called global. 
Remark 7.1. The definition of global asymptotic stability can be given in terms of existence of a function $\beta \in \mathcal{K} \mathcal{L}$ such that $\left\|x\left(t, x_{0}\right)\right\| \leq$ $\beta\left(\left\|x_{0}\right\|, t\right)$ for all $x_{0} \in C_{[-\tau, 0]}$ and $t \geq 0$. In such a case, if $\beta(s, t)=c s e^{-a t}$ for some $c \geq 1$ and $a>0$, then the system (7.1) is called globally exponentially stable.

Similarly as in Section 2, the property of nearly FxTS is conceptually different from FxTS notion, since in the former case $\sup _{\varrho>0} T_{\varrho} \leq+\infty$, i.e., at the origin a nearly FxTS system (7.1) may be just asymptotically stable. On other words, a FxTS system has to be FTS and nearly FxTS at the origin simultaneously. The difference between nearly FxTS and asymptotically stable systems becomes important for an unbounded set $\Omega$ only.

Note that due to uniqueness of solutions of (7.1), for any $x_{0} \in \Omega$ and $t \in \mathbb{R}_{+}$:

$$
T\left(x_{t}\right)=\max \left\{T\left(x_{0}\right)-t, 0\right\},
$$

which can be shown by repeating the arguments of (Bhat and Bernstein, 2000).

In (Efimov et al., 2014b) a necessary condition of finite-time stability of (7.1) is discussed, which is based on the observation that

$$
x\left(t, x_{0}\right)=0 \quad \forall t \geq T\left(x_{0}\right) \Rightarrow f\left(x_{t}\right)=0 \quad \forall t \geq T\left(x_{0}\right),
$$

but $x_{T\left(x_{0}\right)} \neq \mathbf{0}$ according to Definition 7.1 (we need also continuity of $f$ here). Recall that initially it has been assumed only $f(\mathbf{0})=0$, but the observation above necessary leads to a more strong restriction: there exists a nonempty set $\Omega_{0} \subseteq \Omega \cap C_{[-\tau, 0]}^{0}$, where

$$
C_{[-\tau, 0]}^{0}=\left\{\phi \in C_{[-\tau, 0]}: \phi(0)=0\right\},
$$

such that

$$
f(\phi)=0 \quad \forall \phi \in \Omega_{0} .
$$

If we will consider the limit case with

$$
\Omega_{0}=\Omega \cap C_{[-\tau, 0]}^{0},
$$

which is assumed in the sequel, then several results discovered in FTS systems described by ODEs can be recovered for (7.1). Note that in such a case we have

$$
T\left(x_{0}\right)=0 \quad \forall x_{0} \in \Omega_{0}
$$


provided that solutions are unique in the forward time.

Proposition 7.1. If solutions of the FTS system (7.1) depend continuously of initial conditions uniformly on compact intervals of time, i.e., for any finite $\delta>0$

$$
\sup _{t \in[0, \delta]}\left\|x\left(t, x^{*}\right)-x\left(t, x_{0}\right)\right\| \rightarrow 0 \text { as } x^{*} \rightarrow x_{0} \in \Omega \backslash \Omega_{0}
$$

then $T \in C\left(\Omega, \mathbb{R}_{+}\right)$provided that $T$ is continuous at any point of the set $\Omega_{0}$.

Proof. Take $x_{0} \in \Omega \backslash \Omega_{0}$ and an arbitrary sequence $x_{i} \in \Omega, i \in \mathbb{N}$ converging to $x_{0}$. For the solutions $x\left(t, x_{i}\right)$, denote by $x_{t}^{i}$ the state of the system (7.1) at time instant $t$ with the initial condition $x_{i}\left(x_{t}=x_{t}^{0}\right.$ as before).

On the one hand, from the definition of the settling-time functional we derive that $x_{T\left(x_{0}\right)} \in \Omega_{0}$. On the other hand, the continuous dependence of solutions on initial conditions implies

$\lim _{i \rightarrow+\infty} x\left(T\left(x_{0}\right), x_{i}\right)=x\left(T\left(x_{0}\right), x_{0}\right)=0$ and $\lim _{i \rightarrow+\infty}\left\|x_{T\left(x_{0}\right)}^{i}-x_{T\left(x_{0}\right)}\right\|=0$.

Hence, the continuity of $T$ at $x_{T\left(x_{0}\right)}$ implies

$$
\lim _{i \rightarrow+\infty} T\left(x_{T\left(x_{0}\right)}^{i}\right)=T\left(x_{T\left(x_{0}\right)}\right)=0 .
$$

Then due to (7.2) we have

$$
0=\lim _{i \rightarrow+\infty} T\left(x_{T\left(x_{0}\right)}^{i}\right)=\lim _{i \rightarrow+\infty} \max \left\{T\left(x_{i}\right)-T\left(x_{0}\right), 0\right\}
$$

i.e., $\lim \sup _{i \rightarrow+\infty} T\left(x_{i}\right) \leq T\left(x_{0}\right)$.

Let $T^{-}=\liminf _{i \rightarrow+\infty} T\left(x_{i}\right)$. Then there exists a sub-sequence $x_{i_{j}}$ such that $\lim _{j \rightarrow+\infty} T\left(x_{i_{j}}\right)=T^{-}$. Since due to continuous dependence of solutions on initial conditions and on the time argument we have $\left|x\left(T\left(x_{i_{j}}\right), x_{i_{j}}\right)-x\left(T^{-}, x_{0}\right)\right| \leq\left|x\left(T\left(x_{i_{j}}\right), x_{i_{j}}\right)-x\left(T^{-}, x_{i_{j}}\right)\right|+\mid x\left(T^{-}, x_{i_{j}}\right)-$ $x\left(T^{-}, x_{0}\right) \mid \rightarrow 0$ as $j \rightarrow+\infty$ then

$$
0=\lim _{j \rightarrow+\infty} x\left(T\left(x_{i_{j}}\right), x_{i_{j}}\right)=x\left(T^{-}, x_{0}\right) .
$$


The latter means $x_{T^{-}} \in \Omega_{0}$ and $T^{-} \geq T\left(x_{0}\right)$ by the definition of $T$, i.e., $\liminf _{i \rightarrow+\infty} T\left(x_{i}\right)=T^{-} \geq T\left(x_{0}\right)$.

Combining the estimates for lim inf and lim sup we conclude $T\left(x_{i}\right) \rightarrow$ $T\left(x_{0}\right)$ as $i \rightarrow+\infty$.

Note that the requirement on continuity of $T$ at any point of the set $\Omega_{0}$ imposed in the last proposition means a local stability of the set $\Omega_{0}$ for the solutions of (7.1). Indeed, in such a case the properties of $T$ imply existence of some $\varrho_{1}, \varrho_{2} \in \mathcal{K}_{\infty}$ such that $\varrho_{1}\left(\|x\|_{\Omega_{0}}\right) \leq T(x) \leq \varrho_{2}\left(\|x\|_{\Omega_{0}}\right)$ for all $x$ in a vicinity $\mathcal{O}$ of $\Omega_{0}$, where $\|x\|_{\Omega_{0}}=\inf _{\phi \in \Omega_{0}}\|x-\phi\|$. Hence, take any $x_{0} \in \mathcal{O}$, from (7.2) we have:

$$
\varrho_{1}\left(\left\|x_{t}\right\|_{\Omega_{0}}\right) \leq T\left(x_{t}\right) \leq T\left(x_{0}\right) \leq \varrho_{2}\left(\left\|x_{0}\right\|_{\Omega_{0}}\right)
$$

for all $t \in\left[0, T\left(x_{0}\right)\right.$ ), which implies the stability of $\Omega_{0}$ (Kolmanovsky and Nosov, 1986).

Corollary 7.1. Let $f$ in the FTS system (7.1) satisfy the property (7.3) and for any bounded set $X \in \Omega \backslash \Omega_{0}$, there exist $L>0$ such that $\|f(\phi)-f(\varphi)\| \leq L\|\phi-\varphi\|$ for all $\phi, \varphi \in X$, then $T \in C\left(\Omega, \mathbb{R}_{+}\right)$ provided that $T$ is continuous at any point of the set $\Omega_{0}$.

Proof. It is enough to show that under introduced hypotheses the solutions of (7.1) depend continuously in initial conditions uniformly on compact intervals of time $t \geq 0$. Integrating (7.1) for any $x_{1}, x_{2} \in \Omega \backslash \Omega_{0}$ on the interval $t \in\left[0, T_{12}\right)$, where $T_{12}=\min \left\{T\left(x_{1}\right), T\left(x_{2}\right)\right\}$, for the respective solutions $x_{t}^{x_{1}}, x_{t}^{x_{2}}$ we obtain:

$$
x\left(t, x_{i}\right)=x_{i}(0)+\int_{0}^{t} f\left(x_{s}^{x_{i}}\right) d s, i=1,2 .
$$

Since the solutions $x_{t}^{x_{1}}, x_{t}^{x_{2}}$ belong to a bounded set $\mathcal{X} \subset \Omega$ due to the FTS, and using the Lipschitz property of $f$, there exists $L>0$ on $\mathcal{X} \backslash \Omega_{0}$ such that

$$
\begin{aligned}
\left\|x_{t}^{x_{1}}-x_{t}^{x_{2}}\right\| & \leq\left\|x_{1}-x_{2}\right\|+\int_{0}^{s}\left\|f\left(x_{s}^{x_{1}}\right)-f\left(x_{s}^{x_{2}}\right)\right\| d s \\
& \leq\left\|x_{1}-x_{2}\right\|+L \int_{0}^{t}\left\|x_{s}^{x_{1}}-x_{s}^{x_{2}}\right\| d s
\end{aligned}
$$


for any $t \in\left[0, T_{12}\right)$. Denote

$$
m(t)=\left\|x_{1}-x_{2}\right\|+L \int_{0}^{t}\left\|x_{s}^{x_{1}}-x_{s}^{x_{2}}\right\| d s,
$$

then

$$
\frac{d m(t)}{d t}=L\left\|x_{t}^{1}-x_{t}^{2}\right\| \leq L m(t)
$$

from which we derive

$$
\left\|x_{t}^{x_{1}}-x_{t}^{x_{2}}\right\| \leq m(t) \leq e^{L t} m(0)=e^{L t}\left\|x_{1}-x_{2}\right\|
$$

for all $t \in\left[0, T_{12}\right)$, which implies the desired continuity in initial conditions uniformly on $\left[0, T_{12}\right]$. For $t \in\left[T_{12}, T^{12}\right)$, where $T^{12}=\max \left\{T\left(x_{1}\right), T\left(x_{2}\right)\right\}$, we get:

$$
\begin{aligned}
\left\|x_{t}^{x_{T_{12}}^{x_{1}}}-x_{t}^{x_{T_{12}}^{x_{2}}}\right\| \leq & \left\|x_{T_{12}}^{x_{1}}-x_{T_{12}}^{x_{2}}\right\|+\int_{T_{12}}^{T^{12}}\left\|f\left(x_{s}^{x_{T_{12}}^{x_{1}}}\right)-f\left(x_{s}^{x_{T_{12}}^{x_{2}}}\right)\right\| d s \\
& \leq\left\|x_{T_{12}}^{x_{1}}-x_{T_{12}}^{x_{2}}\right\|+\int_{T_{12}}^{T^{12}} M d s,
\end{aligned}
$$

where $M=\sup _{t \in\left[T_{12}, T^{12}\right)}\left\|f\left(x_{t}^{x_{T_{12}}^{x_{1}}}\right)-f\left(x_{t}^{x_{T_{12}}^{x_{2}}}\right)\right\|$. Note that $T_{12}, T^{12}$ and $M$ admit uniform upper bounds on $\mathcal{X}$. Finally,

$$
\begin{gathered}
\left\|x_{t}^{x_{T_{12}}^{x_{1}}}-x_{t}^{x_{T_{12}}^{x_{2}}}\right\| \leq\left\|x_{T_{12}}^{x_{1}}-x_{T_{12}}^{x_{2}}\right\|+M\left|T\left(x_{1}\right)-T\left(x_{2}\right)\right| \\
\leq e^{L T_{12}}\left\|x_{1}-x_{2}\right\|+M \max \left\{T\left(x_{T_{12}}^{x_{1}}\right), T\left(x_{T_{12}}^{x_{2}}\right)\right\},
\end{gathered}
$$

and recall that $\inf _{\phi \in \Omega_{0}}\left\|x_{T_{12}}^{x_{i}}-\phi\right\| \rightarrow 0$ as $x_{1} \rightarrow x_{2}$ for $i=1,2$, i.e., $x_{T_{12}}^{x_{i}}$ approaching $\Omega_{0}$ if the distance between $x_{1}$ and $x_{2}$ is decreasing, then $\max \left\{T\left(x_{T_{12}}^{x_{1}}\right), T\left(x_{T_{12}}^{x_{2}}\right)\right\} \rightarrow 0$ as $x_{1} \rightarrow x_{2}$ due to assumed continuity of $T$ on $\Omega_{0}$. Obviously,

$$
\left\|x_{t}^{x^{x_{1}}}-x_{t}^{x^{x_{2}}} T^{12}\right\|=0
$$

for all $t \geq T^{12}$, and the required continuity of solutions of (7.1) in the initial conditions uniformly on compact intervals of time is established.

Next, the proof repeats the substantiation of Proposition 7.1.

It has been also observed that for time-delay systems the convergence rates, which are usual for ODEs, can be rare, while a more natural accelerated convergence is hyperexponential (Polyakov et al., 2015b) (that is faster than any exponential estimate). 


\subsubsection{Application of Lyapunov-Krasovskii approach}

For introduced stability notions of (7.1) there exist the following sufficient conditions:

Proposition 7.2. Let the system (7.1) admit $V \in C\left(\Omega, \mathbb{R}_{+}\right), \eta_{1}, \eta_{2} \in$ $\mathcal{K}_{\infty}, \rho \in \mathcal{K}$ and $\epsilon>0$ such that $\dot{z}(t)=-\rho(z(t))$ has a flow for all $z(0) \in \mathbb{R}_{+}$and $t \in \mathbb{R}_{+}$, with

$$
\eta_{1}(\|\phi(0)\|) \leq V(\phi) \leq \eta_{2}(\|\phi\|), D^{+} V(\phi) \leq-\rho(V(\phi))
$$

for all $\phi \in \Omega$.

If

$$
\int_{0}^{\epsilon} \frac{d z}{\rho(z)}<+\infty
$$

then the system (7.1) is FTS at the origin with the settling time satisfying an upper estimate:

$$
T(\phi) \leq \int_{0}^{V(\phi)} \frac{d z}{\rho(z)} .
$$

If

$$
\int_{\epsilon}^{\sup _{\phi \in \Omega} V(\phi)} \frac{d z}{\rho(z)}<+\infty
$$

then the system (7.1) is nearly FxTS, and it is FxTS at the origin if $\epsilon=0$.

Proof. The FTS and nearly FxTS cases were proven in (Moulay et al., 2008) and (Efimov and Aleksandrov, 2020), respectively. The FxTS result follows from their combination.

A usual example of such a function $\rho$ providing FTS property includes

$$
\rho(z)=a z^{\alpha}
$$

for $a>0$ and $\alpha \in[0,1)$. Then it is straightforward to check that under the conditions of Proposition 7.2, the solutions of system (7.1) admit 
an upper estimate for $x_{0} \in \Omega$ and all $t \geq 0$ :

$$
\left\|x\left(t, x_{0}\right)\right\| \leq \max \left\{0, \eta_{1}^{-1} \circ\left(\eta_{2}^{1-\alpha}\left(\left\|x_{0}\right\|\right)-a(1-\alpha) t\right)^{\frac{1}{1-\alpha}}\right\},
$$

hence, the system is FTS with

$$
T\left(x_{0}\right) \leq \frac{\eta_{2}^{1-\alpha}\left(\left\|x_{0}\right\|\right)}{a(1-\alpha)} .
$$

If $\alpha=1$, then the system is asymptotically stable:

$$
\left\|x\left(t, x_{0}\right)\right\| \leq \eta_{1}^{-1}\left(\eta_{2}\left(\left\|x_{0}\right\|\right) \exp (-a t)\right)
$$

for all $t \geq 0$ and $x_{0} \in \Omega$. Finally, if $\alpha>1$, then (7.1) is nearly FxTS:

$$
\left\|x\left(t, x_{0}\right)\right\| \leq \eta_{1}^{-1}\left(\frac{1}{\left(\eta_{2}^{1-\alpha}\left(\left\|x_{0}\right\|\right)+a(\alpha-1) t\right)^{\frac{1}{\alpha-1}}}\right)
$$

for all $t \geq 0$ and $x_{0} \in \Omega$. A choice

$$
\rho(z)=a\left(z^{\alpha}+z^{\beta}\right)
$$

with $a>0, \alpha \in[0,1)$ and $\beta>1$ is conventional for FxTS as before.

Note that comparing with the results for ODEs, the conditions of Proposition 7.2 do not imply continuity of the settling-time function (since there is no statement that continuity at the origin implies this property globally for $T$ ), however recalling the conditions of Proposition 7.1 that results can be recovered for time-delay systems:

Theorem 7.2. Let solutions of the system (7.1) depend continuously of initial conditions uniformly on compact intervals of time, then the origin of (7.1) is FTS with a continuous settling-time functional $T$ if and only if there exist $V \in C\left(\Omega, \mathbb{R}_{+}\right), \eta_{1}, \eta_{2} \in \mathcal{K}_{\infty}, c>0$ and $\alpha \in(0,1)$ such that

$$
\eta_{1}(\|\phi(0)\|) \leq V(\phi) \leq \eta_{2}(\|\phi\|), D^{+} V(\phi) \leq-c V^{\alpha}(\phi)
$$

for all $\phi \in \Omega$. 
Proof. For the FTS case, existence of such a Lyapunov-Krasovskii functional $V$ implies this stability property ( $V$ is positive definite with respect to the set $\Omega^{0}$ ) and continuity of $T$ follows from the inequality $T(\phi) \leq \frac{V^{1-\alpha}(\phi)}{(1-\alpha) c}$ for all $\phi \in \Omega$. Conversely, if the system is FTS, take $V(\phi)=\frac{T^{1+\gamma}(\phi)}{1+\gamma}$ for some $\gamma>0$, then $\eta_{1}(s)=\inf _{\|\phi(0)\|=s} V(\phi), \eta_{2}(s)=$ $\sup _{\|\phi\| \leq s} V(\phi)$ and

$$
D^{+} V(\phi)=T^{\gamma}(\phi) D^{+} T(\phi)
$$

for any $\phi \in \Omega$. Recall (7.2), then $D^{+} T(\phi)=-1$ for $\phi \in \Omega \backslash \Omega^{0}$ and $D^{+} T(\phi)=0$ for $\phi \in \Omega^{0}$, hence

$$
D^{+} V(\phi) \leq-c V^{\alpha}(\phi)
$$

for all $\phi \in \Omega$, where $c=(1+\gamma)^{\alpha}$ and $\alpha=\frac{\gamma}{1+\gamma} \in(0,1)$ as needed.

Example 7.1. Let us show that the restriction $\Omega_{0}=\Omega \cap C_{[-\tau, 0]}^{0}$ used in Proposition 7.1 and Theorem 7.2 is indeed important. Consider

$$
\dot{x}(t)=-2 x^{\frac{1}{3}}(t)-\left(\max _{\theta \in[-\tau, 0]} x(t+\theta)\right)^{\frac{1}{3}},
$$

where $x(t) \in \mathbb{R}$. It is easy to check that the system is globally asymptotically stable using the Lyapunov-Razumikhin function $V(x)=\|x\|$. Moreover, it is also globally FTS. Indeed, if $x(\theta) \leq 0$ for all $\theta \in$ $[-\tau, 0]$, then at the instant $t^{\prime} \geq 0$ such that $x\left(t^{\prime}\right)=0$ for the first time (such an instant exists since $\dot{V}(t) \leq-2 V^{\frac{1}{3}}(t)$ for all $t \in\left[0, t^{\prime}\right]$ ) we have that $\max _{\theta \in[-\tau, 0]} x\left(t^{\prime}+\theta\right)=0$ (i.e., $\dot{x}\left(t^{\prime}\right)=0$ ) and, hence, $\max _{\theta \in[-\tau, 0]} x(t+\theta)=0$ for all $t \in\left[t^{\prime}, t^{\prime}+\tau\right]$ and actually $T\left(x_{0}\right)=t^{\prime}$. If $\max _{\theta \in[-\tau, 0]} x(\theta)>0$, then there exists $t^{\prime} \geq 0$ such that $x\left(t^{\prime}\right)=0$ for the first time (such an instant exists by the same argument), $\dot{x}\left(t^{\prime}\right)<0$ due to the equation of the system, and $x(t) \leq 0$ for all $t \geq t^{\prime}$, which implies next the finite-time convergence by the reasoning given for the previous case.

Note that this system is continuous and homogeneous of a negative degree in the sens of (Efimov et al., 2014a), the property (7.3) holds for $\Omega_{0}=\left\{\phi \in C_{[-\tau, 0]}^{0}: \phi(\theta) \leq 0, \theta \in[-\tau, 0]\right\} \subset \Omega \cap C_{[-\tau, 0]}^{0}$ (the conditions of Proposition 7.1 are not satisfied), and the settling-time functional is 
discontinuous at $\Omega_{0}$. To check this we can consider any arbitrary small $x_{0} \in \Omega \backslash \Omega^{0}$ with $x(\theta)>0$ for all $\theta \in[-\tau, 0]$ whose settling time cannot be smaller than $\tau$ (the trajectory $x(t)$ has to become negative for all $[t-\tau, t]$ before a convergence till the origin can emerge).

An extension of the converse results for (nearly) FxTS requires additional investigations, since an application of the arguments used in the ODE case requires the concept of solutions in the inverse time to be defined, which is not developed for time-delay systems.

\subsubsection{Application of Lyapunov-Razumikhin approach}

For the Lyapunov-Krasovskii approach there are converse results for asymptotic stability (Pepe and Karafyllis, 2013; Pepe et al., 2017; Efimov and Fridman, 2020), while the Lyapunov-Razumikhin method is only sufficient (Kolmanovsky and Nosov, 1986). In addition, evaluations by the Lyapunov-Razumikhin approach of the convergence rates requires substantial modifications of its formulation:

Proposition 7.3. (Myshkis, 1995) Let there exist a locally Lipschitz continuous Lyapunov-Razumikhin function $V: \mathbb{R}^{n} \rightarrow \mathbb{R}_{+}$such that

(i) for some $\alpha_{1}, \alpha_{2} \in \mathcal{K}_{\infty}$ and all $x \in \mathbb{R}^{n}$ :

$$
\alpha_{1}(|x|) \leq V(x) \leq \alpha_{2}(|x|) ;
$$

(ii) for some $\gamma^{\prime}>1, \alpha^{\prime}>0$ and all $\varphi \in C_{[-\tau, 0]}$ :

$$
\begin{gathered}
\max _{\theta \in[-\tau, 0]} V(\varphi(\theta)) \leq \gamma^{\prime} V(\varphi(0)) \Rightarrow \\
D^{+} V(\varphi(0)) f(\varphi) \leq-\alpha^{\prime} V(\varphi(0)) .
\end{gathered}
$$

Then the origin is globally asymptotically stable for the system (7.1) with exponential rate of convergence, and for all $x_{0} \in C_{[-\tau, 0]}$ and $t \geq 0$ :

$$
\left|x\left(t, x_{0}\right)\right| \leq \alpha_{1}^{-1}\left(\exp \left(-\min \left\{\alpha^{\prime}, \frac{\ln \gamma^{\prime}}{\tau}\right\} t\right) \alpha_{2}\left(\left\|x_{0}\right\|\right)\right) .
$$


A similar estimate can be derived from Halanay's inequality (Halanay, 1966).

The above result is given to recall the formulation of the LyapunovRazumikhin approach: if we replace constants $\gamma^{\prime}, \alpha^{\prime}$ with function $\gamma^{\prime}, \alpha^{\prime} \in \mathcal{K}$ of $V$, such that $\gamma^{\prime}(s)>s$ for all $s>0$, then the conventional sufficient Lyapunov-Razumikhin condition for asymptotic stability of (7.1) is recovered (Kolmanovsky and Nosov, 1986). The formulations for accelerated convergence rates are more complex:

Theorem 7.3. (Efimov and Aleksandrov, 2020) Let there exist a locally Lipschitz continuous Lyapunov-Razumikhin function $V$ : $\mathbb{R}^{n} \rightarrow \mathbb{R}_{+}$with (7.5) for some $\alpha_{1}, \alpha_{2} \in \mathcal{K}_{\infty}$ and all $x \in \mathbb{R}^{n}$, and one of the following properties is satisfied:

(i) for some $\mu \in(0,1), c>0, \alpha^{\prime}>0$ and all $\varphi \in C_{[-\tau, 0]}$ :

$$
\begin{gathered}
\max _{\theta \in[-\tau, 0]} V^{1-\mu}(\varphi(\theta)) \leq V^{1-\mu}(\varphi(0))+c \tau \Rightarrow \\
D^{+} V(\varphi(0)) f(\varphi) \leq-\alpha^{\prime} V^{\mu}(\varphi(0)),
\end{gathered}
$$

then the origin is globally FTS for the system (7.1), and for all $x_{0} \in C_{[-\tau, 0]}$ and $t \geq 0$ :

$$
\begin{gathered}
\left|x\left(t, x_{0}\right)\right| \leq \max \left\{0, \alpha_{1}^{-1} \circ\left(\alpha_{2}^{1-\mu}\left(\left\|x_{0}\right\|\right)\right.\right. \\
\left.\left.-\min \left\{\alpha^{\prime}(1-\mu), c\right\} t\right)^{\frac{1}{1-\mu}}\right\} ;
\end{gathered}
$$

(ii) for some $\mu>1, c>0, \alpha^{\prime}>0$ and all $\varphi \in C_{[-\tau, 0]}$ :

$$
\begin{gathered}
\frac{1}{\left(\max _{\theta \in[-\tau, 0]} V(\varphi(\theta))\right)^{1-\mu}+c \tau} \leq V^{\mu-1}(\varphi(0)) \Rightarrow \\
D^{+} V(\varphi(0)) f(\varphi) \leq-\alpha^{\prime} V^{\mu}(\varphi(0)),
\end{gathered}
$$

then the system (7.1) is globally nearly FxTS at the origin, and for all $x_{0} \in C_{[-\tau, 0]}$ and $t \geq 0$ :

$$
\left|x\left(t, x_{0}\right)\right| \leq \alpha_{1}^{-1}\left(\frac{1}{\left(\alpha_{2}^{1-\mu}\left(\left\|x_{0}\right\|\right)+\min \left\{\alpha^{\prime}(\mu-1), c\right\} t\right)^{\frac{1}{\mu-1}}}\right) .
$$


As we can conclude from the results of Proposition 7.3 and Theorem 7.3, the Lyapunov-Razumikhin approach can be used for estimation of the rate of convergence, but the conditions have to be formulated differently in accordance with the kind of decay.

Example 7.2. (Efimov and Aleksandrov, 2020) Consider a scalar example with $V(t) \in \mathbb{R}_{+}$for all $t \geq 0$, and $V_{0} \in C_{[-\tau, 0]}$ with $\left\|V_{0}\right\| \leq 1$ :

$$
\dot{V}(t) \leq-a V^{\mu}(t)+b V^{\eta}(t) V^{\rho}(t-\tau),
$$

where $a>0, b>0, \mu \in(0,1), \rho>0$ and $\eta \geq \mu$ are parameters. For $c>0$ the Lyapunov-Razumikhin relation is leads to:

$$
\begin{gathered}
V^{1-\mu}(t-\tau)<V^{1-\mu}(t)+c \tau \Rightarrow \\
\dot{V}(t) \leq-a V^{\mu}(t)+b V^{\eta}(t)\left(V^{1-\mu}(t)+c \tau\right)^{\frac{\rho}{1-\mu}} .
\end{gathered}
$$

Applying Jensen's inequality,

$$
\begin{gathered}
\left(V^{1-\mu}(t)+c \tau\right)^{\frac{\rho}{1-\mu}} \leq\left(V^{\rho}(t)+(c \tau)^{\frac{\rho}{1-\mu}}\right) \\
\quad \times \begin{cases}1 & \rho \in(0,1-\mu] \\
2^{\frac{\rho}{1-\mu}-1} & \rho>1-\mu\end{cases}
\end{gathered}
$$

we obtain

$$
\begin{gathered}
V^{1-\mu}(t-\tau)<V^{1-\mu}(t)+c \tau \Rightarrow \\
\dot{V}(t) \leq-a V^{\mu}(t)+\max \left\{1,2^{\frac{\rho}{1-\mu}-1}\right\} b \\
\times\left[V^{\eta+\rho}(t)+V^{\eta}(t)(c \tau)^{\frac{\rho}{1-\mu}}\right] \\
\leq-\left[a-\max \left\{1,2^{\frac{\rho}{1-\mu}-1}\right\} b\left(1+(c \tau)^{\frac{\rho}{1-\mu}}\right)\right] V^{\mu}(t),
\end{gathered}
$$

and the conditions for FTS of Theorem 7.3 are verified locally for

$$
a>\max \left\{1,2^{\frac{\rho}{1-\mu}-1}\right\} b\left(1+(c \tau)^{\frac{\rho}{1-\mu}}\right) .
$$

Let $V_{0} \in C_{[-\tau, 0]}$ in

$$
\dot{V}(t) \leq-a V^{\mu}(t)+\frac{b V(t) V^{\mu-1}(t-\tau)}{1+c \tau V^{\mu-1}(t-\tau)},
$$


where $a>0, b>0, \mu>1, c>0$ are parameters. The LyapunovRazumikhin relation implies:

$$
\begin{gathered}
\frac{1}{V^{1-\mu}(t-\tau)+c \tau}<V^{\mu-1}(t) \Rightarrow \\
\dot{V}(t) \leq-(a-b) V^{\mu}(t),
\end{gathered}
$$

and the conditions of Theorem 7.3 are verified for

$$
a>b .
$$

Example 7.3. Note that an FTS system (7.4) does not satisfy the conditions of Theorem 7.3, which is not a contradiction since it admits a discontinuous settling-time functional, while the estimate given in Theorem 7.3 implies its continuity.

\subsubsection{Discussion}

As we can conclude from the results of this section, FTS is not a natural type of behavior of time-delay systems, since the influence of past values of the state may block a finite-time settling of the trajectories at the origin, then additional structural conditions are needed. Control design approaches for finite-time stabilization of time-delay systems can be found in (Moulay et al., 2008; Polyakov et al., 2015b; Nekhoroshikh et al., 2020), and the simplest ways of obtaining accelerated regulation in this class of systems is by using prediction techniques to compensate the delays as in (Karafyllis, 2006), the theory of homogeneity (Zimenko et al., 2017; Zimenko et al., 2019) or the domination approach (Wang et al., 2020b).

\subsection{Partial differential equations and evolution models}

The proofs of all claims given in this section can be found in Polyakov, 2020 .

\subsubsection{Stability of evolution systems}

The notions of finite-time and fixed-time stability of evolution systems are similar to the case of ODEs. Below we denote $x\left(t, x_{0}\right)$ a solution of 
the system (3.25), i.e., $\dot{x}=A x+f(x)$, where $x_{0}$ indicates the initial condition of the system and means

$$
x\left(0, x_{0}\right)=x_{0} .
$$

Definition 7.2 (Lyapunov stability). The origin of a dynamical system is said to be locally (globally) Lyapunov stable if there exists $\varepsilon \in \mathcal{K}\left(\right.$ resp. $\left.\varepsilon \in \mathcal{K}_{\infty}\right)$ such that

$$
\left\|x\left(t, x_{0}\right)\right\| \leq \varepsilon\left(\left\|x_{0}\right\|\right), \quad t>t_{0}
$$

for any solution $x\left(t, x_{0}\right)$ of $(3.25)$ and any $x_{0} \in U$, where $U \subset \mathbb{H}$ is a neighborhood of the origin (resp. $U=\mathbb{H})$.

If the origin of the system is unstable if it does not satisfy Definition 7.2. The following result is well-known for ODE Bhat and Bernstein, 2000 and holds for evolution systems as well.

Proposition 7.4. If the origin of a dynamical system is Lyapunov stable then $x(t, \mathbf{0}) \equiv \mathbf{0}$ is the unique solution of the system (3.25) with the initial condition $x(0)=\mathbf{0}$.

Definition 7.3 (Finite-time stability). The origin of a dynamical system is said to be locally (globally) finite-time stable if it is locally (globally) Lyapunov stable and finite-time attractive in an open neighborhood $U$ of the origin (resp. $U=\mathbb{H}$ ), i.e., $\exists T: \mathbb{H} \rightarrow \mathbb{R}_{+}$ such that

$$
\forall x_{0} \in U \quad \Rightarrow \quad x\left(t, x_{0}\right)=\mathbf{0}, \quad \forall t \geq T\left(x_{0}\right)
$$

for any solution $x\left(t, x_{0}\right)$ of the system. The set $U$ is called a domain of finite-time attraction.

Obviously, if $T$ satisfies the latter definition then the function $T+T^{+}$ also does for any nonnegative $T^{+}$. Therefore, it is reasonable to consider a minimal functional $T$. 
Definition 7.4. A functional $T: \mathbb{H} \rightarrow \mathbb{R}_{+}$is called the settling-time function of the finite-time stable system, if $T$ satisfies Definition 7.3 and the functional $T-\tilde{T}$ does not satisfy Definition 7.3 (with the same $U$ ) for any $\tilde{T}: \mathbb{H} \rightarrow \mathbb{R}_{+}: \tilde{T} \neq \mathbf{0}$.

Finite-time stability always implies asymptotic stability. The settlingtime function $T$ of time-invariant finite-time stable system is independent of $t_{0}$. However, in contrast to asymptotic and Lyapunov stability, finitetime stability of time-invariant system, in general, does not imply uniform finite-time stability, which requires at least boundedness of the settling-time function in a neighborhood of the origin (see Bhat and Bernstein, 2000, page 756).

Definition 7.5 (Uniform finite-time stability). The origin of a dynamical system is said to be locally (globally) uniformly finite-time stable if it is finite-time stable with $U \subset \mathbb{H}$ (resp. $U=\mathbb{H}$ ) and the settling time function $T: U \rightarrow \mathbb{R}$ is locally bounded, i.e.,

$$
\forall y \in U, \quad \exists \varepsilon>0 \quad \text { such that } \sup _{\left\|x_{0}-y\right\|<\varepsilon} T\left(x_{0}\right)<+\infty .
$$

Definition 7.6 (Fixed-time stability). The origin of a dynamical system is said to be locally (globally) fixed-time stable if it is locally (globally) uniformly finite-time stable with $U \subset \mathbb{H}$ (resp. $U=\mathbb{H}$ ) and the settling time function $T$ is bounded on $U$, i.e.,

$$
\exists T_{\max }>0: x\left(t, x_{0}\right)=0, \quad t>t_{0}+T_{\max }, \quad \forall t_{0} \in \mathbb{R}, \quad \forall x_{0} \in U .
$$

It is worth stressing that in the infinite-dimensional case, the fixed-time stability can be discovered even for linear evolution systems.

Example 7.4. Let us consider the wave equation

$$
u_{t t}=u_{x x}, \quad t>0, \quad x \in[0,1], \quad u: \mathbb{R}_{+} \times[0,1] \rightarrow \mathbb{R},
$$

with the so-called "transparent" boundary condition (see, e.g., Perrollaz and Rosier, 2014)

$$
u_{x}(t, 0)=u_{t}(t, 0), \quad u_{x}(t, 1)=-u_{t}(t, 1)
$$


and the initial conditions

$$
u(0, x)=\phi(x), \quad u_{t}(0, x)=\psi
$$

from

$$
\left\{(\phi, \psi) \in H^{1}((0,1), \mathbb{R}) \times L^{2}((0,1), \mathbb{R}): \phi(0)+\phi(1)+\int_{0}^{1} \psi(s) d s=0\right\},
$$

where $L^{2}$ and $H^{1}$ are Lebesgue and Sobolev spaces, respectively. The boundary conditions are transparent in the sense that any wave $u(t, x)=$ $f_{1}(x-t)$ traveling to the right leaves the domain at $x=1$ and does not generate any reflected wave. Any wave $u(t, x)=f_{2}(x+t)$ traveling to the left leave the domain at $x=0$ similarly. Since any solution of the wave system is given by $u(t, x)=f_{1}(x-t)+f_{2}(x+t)$ then it vanishes after the time instant $t=1$ independently of the initial condition.

\subsubsection{Lyapunov characterization of finite-time stability of evolution equations}

Let

$$
\bar{D}^{+} \phi(t)=\limsup _{h \rightarrow 0^{+}} \frac{\phi(t+h)-\phi(t)}{h}
$$

denotes the right-hand upper Dini derivative of the function $\phi: \mathbb{R} \rightarrow \mathbb{R}$ at the point $t \in \mathbb{R}$. Using Bolzano-Weierstrass Theorem it can be shown that a finite or infinite Dini derivative exists for any function $\phi: \mathbb{R} \rightarrow \mathbb{R}$ and any point $t \in \mathbb{R}$.

Recall that a function $\varphi:(a, b) \rightarrow \mathbb{R}$ is decreasing on $(a, b), a<b$ if

$$
\forall t_{1}, t_{2} \in(a, b): t_{1} \leq t_{2} \Rightarrow \varphi\left(t_{1}\right) \geq \varphi\left(t_{2}\right) .
$$

From the above definitions we conclude

$$
\phi \text { is decreasing on }(a, b) \quad \Leftrightarrow \quad \bar{D}^{+} \phi(t) \leq 0, \quad \forall t \in(a, b)
$$

Lyapunov function candidates in $\mathbb{R}^{n}$ are positive definite and proper (see, e.g., Clarke et al., 1998). Recall that a mapping $f: \mathbb{R}^{n} \rightarrow \mathbb{R}$ is proper if an inverse image of any compact set is a compact set. In the general case, closedness and boundedness is not sufficient for compactness in Hilbert spaces, and the properness in the classical sense 
may be too strong condition for Lyapunov function candidate. For "generalized" proper functions introduced in Definition 7.7 an inverse image of any compact set belongs to a closed bounded set (which may be not compact in the general case). Below the word "generalized" is omitted for shortness.

Definition 7.7. For a positive definite functional $V: \Omega \subset \mathbb{H} \rightarrow$ $[0,+\infty)$ :

- $V$ is said to be proper at $\mathbf{0}$ (locally proper) if there exists $\underline{V}, \bar{V} \in \mathcal{K}$ such that

$$
\underline{V}(\|x\|) \leq V(x) \leq \bar{V}(\|x\|) \quad \text { for } \quad x \in \Omega \backslash\{\mathbf{0}\},
$$

where $\Omega$ is a neighborhood of $\mathbf{0}$.

- $V$ is said to be globally proper if $\underline{V}, \bar{V} \in \mathcal{K}^{\infty}$.

Theorem 7.4. The origin of the system (3.25) is locally (globally) uniformly finite-time stable with a continuous at the origin settlingtime function if and only if there exists a locally (globally) proper positive definite functional $V: \Omega \subset \mathbb{H} \rightarrow[0,+\infty)$ such that the inequality

$$
\bar{D}^{+} V\left(x\left(t, x_{0}\right)\right) \leq-1, \quad \forall t>0
$$

holds for any solution $x\left(t, x_{0}\right)$ of $(3.25)$ as long as

$$
x\left(t, x_{0}\right) \in \Omega \backslash\{\mathbf{0}\},
$$

where $\Omega$ is a neighborhood of the origin (resp. $\Omega=\mathbb{H}$ ). Moreover, the settling-time of the finite-time stable system (3.25) admits the estimate $T\left(x_{0}\right) \leq V\left(x_{0}\right)$ for all $x_{0} \in U$, where $U \subset \Omega$ is a neighborhood of the origin (resp. $U=\Omega=\mathbb{H}$ ).

To prove the necessity, the following Lyapunov function $V=V_{0}+T$ can be utilized, where $T$ is the settling-time function and $V_{0}\left(x_{0}\right)=$ $\sup _{\forall t>0}\left\|x\left(t, x_{0}\right)\right\|$. If a solution with $x\left(0, x_{0}\right)=x_{0}$ is not unique then 
the latter supremum has to be taken over all solutions with such initial condition. The proof of sufficiency is rather straightforward.

Under additional restrictions to $V$, the stability analysis can be done using only the operators $A$ and $f$ in the right-hand side of (3.25).

Corollary 7.5. Let the system (3.25) have a classical solution for any $x_{0} \in \mathcal{D}(A)$ and all mild solutions of the system depend continuously of initial conditions on compact intervals of time. If there exists a locally (globally) proper locally Lipschitz continuous positive definite functional $V: \mathbb{H} \backslash\{\mathbf{0}\} \rightarrow \mathbb{R}$ such that $V$ is Frechét differentiable on $\mathcal{D}(A) \cap \Omega \backslash\{\mathbf{0}\}$ and

$$
D V(x)(A x+f(x)) \leq-1, \quad \forall x \in \Omega \cap \mathcal{D}(A) \backslash\{\mathbf{0}\},
$$

then the origin of the system (3.25) is locally (globally) uniformly finite-time stable with some finite-time attraction domain $U \subset \Omega$, and the settling time $T\left(x_{0}\right)$ admits the estimate

$$
T\left(x_{0}\right) \leq V\left(x_{0}\right), \quad \forall x_{0} \in U,
$$

where $\Omega$ and $U \subset \Omega$ are some neighborhoods of the origin (resp. $U=\Omega=\mathbb{H})$ and $D V(x) \in \mathcal{L}(\mathbb{H}, \mathbb{H})$ denotes the Frechét derivative of $V$ at the point $x \in \mathcal{D}(A)$.

This result follows from Theorem 7.4 and the identity

$$
\frac{d}{d t} V\left(x\left(t, x_{0}\right)\right)=\left.D V(x)(A x+f(x))\right|_{x=x\left(t, x_{0}\right)},
$$

which holds for any classical solution of (3.25).

Example 7.5. Let us consider the evolution equation

$$
\dot{x}=A x+f(x), \quad t>0, \quad x(0)=x_{0} \in \mathbb{H},
$$

where

$$
f(x)=-\frac{x}{\|x\|^{\alpha}}, \quad x \in \mathbb{H}, \quad \alpha \in(0,1), \quad\|x\|=\sqrt{\langle x, x\rangle},
$$

the operator $A: \mathcal{D}(A) \subset \mathbb{H} \rightarrow \mathbb{H}$ generates a strongly continuous semigroup of linear bounded operators on a real Hilbert space $\mathbb{H}$. Assume 
also that $A$ is a dissipative operator, i.e.,

$$
\langle A x, x\rangle \leq 0, \quad x \in \mathcal{D}(A) .
$$

The functional $V: \mathbb{H} \rightarrow[0,+\infty)$ given by

$$
V(x)=\frac{1}{\alpha}\|x\|^{\alpha}
$$

is Frechét differentiable on $\mathbb{H} \backslash\{\mathbf{0}\}$ and for any $x \in D(A) \backslash\{\mathbf{0}\}$ we have

$$
\begin{gathered}
\dot{V}(x)=\sup _{y \in F(x)} D V(x)(A x+y)=D V(x)\left(A x-\frac{x}{\|x\|^{\alpha}}\right) \\
=\frac{1}{\|x\|^{2-\alpha}}\left\langle A x-\frac{x}{\|x\|^{\alpha}}, x\right\rangle=\frac{\langle A x, x\rangle}{\|x\|^{2-\alpha}}-1 \leq-1 .
\end{gathered}
$$

The origin of the considered evolution system is globally finite-time stable and the settling-time $T$ admits the estimate $T\left(x_{0}\right) \leq\left\|x_{0}\right\|$ for any $x_{0} \in \mathbb{H}$.

In the infinite-dimensional case, a setting-time function $T$ may be continuous, vanishing, but not proper. This means, in the general case, we cannot assume $V=T$ in Theorem 7.4.

Example 7.6 (Polyakov, 2019). Let $\mathbb{H}=L^{2}((-1,1), \mathbb{R})$ and the operator $A$ in the system (7.8) be defined as follows

$$
A z=\frac{\partial^{2}}{\partial z^{2}}, \quad z \in \mathcal{D}(A)
$$

with the domain

$$
D(A)=H^{2}((-1,1), \mathbb{R}) \cap H_{0}^{1}((-1,1), \mathbb{R}) .
$$

It is easy to see that $\phi_{i}=\sin (\pi i z) \in D(A), z \in[-1,1]$ is an eigenvector of the operator $A$ :

$$
A \phi_{i}=-\lambda_{i} x_{i}, \quad \lambda_{i}=\pi^{2} i^{2}, \quad i=1,2 \ldots
$$

Being an orthonormal basis in the separable Hilbert space $\mathbb{H}$, the eigenvectors $\phi_{i}$ allow us to represent any $y \in \mathcal{D}(A)$ in the form

$$
y=\sum_{i=1}^{\infty} x_{i} \phi(x)
$$


Taking into account $\left\langle\phi_{i}, \phi_{j}\right\rangle=0$ for $i \neq j$ and $\left\langle\phi_{i}, \phi_{i}\right\rangle=1$ we derive

$$
\left\langle\phi_{i}, A y\right\rangle=-\lambda_{i} x_{i}, \quad\left\langle\phi_{i}, f(y)\right\rangle=-\frac{x_{i}}{\sqrt{\langle y, y\rangle}}=-\frac{x_{i}}{\sqrt{\sum_{i=1}^{+\infty} x_{i}^{2}}} .
$$

Therefore, any classical solution of (7.8) admits the representation (7.9), where the time-varying functions $x_{i}$ satisfy the following (infinite) system of ODEs

$$
\dot{x}_{i}=-\left(\lambda_{i}+\frac{1}{\sqrt{\sum_{i=1}^{+\infty} x_{i}^{2}}}\right) x_{i}, \quad i=1,2, \ldots .
$$

Since the operator $A$ is dissipative then using Example 7.5 it can be shown that the considered system is finite-time stable, the settling-time $T$ is continuous at the origin and $T(\mathbf{0})=0$.

Let us consider the sequence of initial conditions $x(0)=y_{0}^{j}=\phi_{j} \in$ $\mathcal{D}(A), j=1,2, \ldots$ Obviously, $\left\|y_{0}^{j}\right\|=1$ for all $j \geq 1$, the corresponding solution $y^{j}$ has the form $y_{j}(t)=x_{j}(t) \phi_{j}$ and $\left\|y_{j}(t)\right\|=x_{j}(t)$, where

$$
\dot{x}_{j}=-\left(\lambda_{j}+\frac{1}{\left|x_{j}\right|}\right) x_{j}, \quad x_{j}(0)=1 .
$$

Simple computations show

$$
T\left(y_{j}\right)=\frac{\ln \left(1+\lambda_{j}\right)}{\lambda_{j}} \rightarrow 0 \quad \text { as } \quad j \rightarrow+\infty .
$$

while $\left\|y_{0}^{j}\right\|=1$ for all $j \geq 1$. The latter means that $\sup _{\left\|x_{0}\right\|=1} T\left(x_{0}\right)=0$, i.e., $T$ is not a proper function and it cannot be utilized as a Lyapunov function in Theorem 7.4.

Corollary 7.6. If under conditions of Corollary 7.5 there exists a locally (globally) proper locally Lipschitz continuous positive definite functional $V: \mathbb{H} \backslash\{\mathbf{0}\} \rightarrow \mathbb{R}$ such that $V$ is Frechét differentiable on $\mathcal{D}(A) \cap \Omega \backslash\{\mathbf{0}\}$ and

$$
D V(x)(A x+f(t, x)) \leq-q\left(1+V^{2}(x)\right), \quad \forall x \in D(A) \cap \Omega \backslash\{\mathbf{0}\}, \forall t \in \mathbb{R},
$$

then the origin of the system (3.25) is locally (globally) uniformly fixed-time stable with an attraction domain $U \subset \Omega($ resp. $U=\mathbb{H})$ 
and the settling time $T\left(x_{0}\right)$ admits the estimate

$$
T\left(x_{0}\right) \leq \frac{\pi}{2 q}
$$

for all $x_{0} \in \mathbb{H}$.

The converse Lyapunov theorem of fixed-time stability is still an open problem in the general case. A particular case (see, Theorem 2.5) is studied in Lopez-Ramirez et al., 2019 for $\mathbb{H}=\mathbb{R}^{n}$, where it is assumed that the settling-time function is, at least, locally proper. This is not the case for an unbounded operator $A$ in (3.25) (see Example 7.6).

Example 7.7. Let us consider the evolution equation

$$
\dot{x}=A x-\frac{x}{\|x\|}-\|x\| x, \quad x \in \mathbb{H}
$$

in a Hilbert space $\mathbb{H}$, where $A$ is assumed to be a dissipative operator, i.e.,

$$
\langle A x, x\rangle \leq 0, \quad \forall x \in \mathcal{D}(A) .
$$

Obviously, selecting

$$
V(x)=\|x\|=\sqrt{\langle x, x\rangle}, \quad x \in \mathbb{H}
$$

we derive

$$
\dot{V}(x)=\frac{\langle\dot{x}, x\rangle}{\|x\|}=\langle A x, x\rangle-1-V^{2}, \quad \forall x \in \mathcal{D}(A) \backslash\{\mathbf{0}\} .
$$

The latter means that the considered system is globally uniformly fixed-time stable and the settling-time admits the estimate

$$
T\left(x_{0}\right) \leq \frac{\pi}{2} .
$$

\subsubsection{Finite-time stabilization of a linear evolution equation in a Hilbert space}

Let us consider the following control system

$$
\begin{gathered}
\dot{x}(t)=A x(t)+B u(x(t)), \quad t>0 \\
x(0)=x_{0},
\end{gathered}
$$


where $A: \mathcal{D}(A) \subset \mathbb{B} \rightarrow \mathbb{B}$ is a (possibly unbounded) closed linear operator with the domain $\mathcal{D}(A)$ dense in $\mathbb{B}, B: \mathbb{X} \rightarrow \mathbb{B}$ is a linear bounded operator, $\mathbb{B}$ is a real Banach space and $\mathbb{X}$ is a normed vector space, $x(t)$ is the system state, $u: \mathbb{B} \rightarrow \mathbb{X}$ is a (locally or globally) bounded feedback, i.e., interior or distributed control.

Theorem 7.7 (Polyakov et al., 2018, Polyakov, 2020). Let $A: \mathcal{D}(A) \subset$ $\mathbb{H} \rightarrow \mathbb{H}$ be a generator of a strongly continuous semigroup $\Phi$ of linear bounded operators on $\mathbb{H}, B: \mathbb{X} \rightarrow \mathbb{H}$ be a linear bounded operator and

A) $\mathbf{d}_{1}$ be a uniformly continuous group of linear bounded operators in $\mathbb{H}$ with the generator $G_{\mathbf{d}_{1}} \in \mathcal{L}(\mathbb{H}, \mathbb{H})$;

B) $\mathbf{d}$ be a strongly continuous strictly monotone linear dilation in $\mathbb{H}$ such that $\mathcal{D}(A) \subset \mathcal{D}\left(G_{\mathbf{d}}\right)$, where $G_{\mathbf{d}}: \mathcal{D}\left(G_{\mathbf{d}}\right) \subset \mathbb{H} \rightarrow \mathbb{H}$ is the generator of the dilation group $\mathbf{d}$;

C) there exist a linear bounded operator $K: \mathbb{H} \rightarrow \mathbb{X}$ such that

$$
\exists \rho>0:\left\langle\left(A+B K+\rho G_{\mathbf{d}}\right) x, x\right\rangle \leq 0, \quad \forall x \in \tilde{\mathcal{D}},
$$

where a set $\tilde{\mathcal{D}} \subset \mathcal{D}(A)$ is dense in $\mathcal{D}(A)$.

D) the operator $A$ be $\mathbf{d}$-homogeneous of a degree $\mu \in \mathbb{R}$ with $\mu>-\beta$ and

$$
\mathbf{d}(s) B K \mathbf{d}_{1}(s)=B K \mathbf{d}(s), \quad \forall s \in \mathbb{R} .
$$

Then the following holds.

- The feedback control $u: \mathbb{H} \rightarrow \mathbb{X}$ defined as

$$
u(x)=\left\{\begin{array}{ccc}
\|x\|_{\mathbf{d}}^{\mu} K \mathbf{d}_{1}\left(-\ln \left(\|x\|_{\mathbf{d}}\right)\right) x & \text { if } & x \neq \mathbf{0}, \\
\mathbf{0} & \text { if } & x=\mathbf{0}
\end{array}\right.
$$

is locally Lipschitz continuous on $\mathbb{H} \backslash\{\mathbf{0}\}$ and Fréchet differentiable on $\mathcal{D}\left(G_{\mathbf{d}}\right) \backslash\{\mathbf{0}\}$. Moreover, 
- if $\beta+\mu>\gamma_{\max }$ then $u$ is continuous at $\mathbf{0} \in \mathbb{H}$;

- if $\beta+\mu \geq \gamma_{\max }$ then

$$
\sup _{\|x\| \leq 1}\|u(x)\| \leq\|K\|
$$

- if $\exists M \geq 1:\|\mathbf{d}(s)\| \leq M e^{\left(-\mu+\gamma_{\text {min }}\right) s}, \forall s>0$ then

$$
\sup _{\|x\| \geq 1}\|u(x)\| \leq M\|K\|,
$$

where $\beta:=\inf _{z \in S \cap \mathcal{D}\left(G_{\mathbf{d}}\right)}\left\langle G_{\mathbf{d}} z, z\right\rangle>0, \gamma_{\min }:=\inf _{z \in S}\left\langle G_{\mathbf{d}_{1}} z, z\right\rangle$ and $\gamma_{\max }:=\sup _{z \in S}\left\langle G_{\mathbf{d}_{1}} z, z\right\rangle$.

- The closed-loop system (7.11)- (7.14) is d-homogeneous of degree $\mu$.

- The closed-loop system (7.11)- (7.14) has

- a unique mild solution defined on $[0,+\infty)$ for any $x_{0} \in \mathbb{H}$;

- a unique locally Lipschitz continuous strong solution defined on $[0,+\infty)$ for any $x_{0} \in \mathcal{D}(A)$.

- a unique classical solution defined on $[0,+\infty)$ for any $x_{0} \in \mathcal{D}(A)$ provided that $B K \mathcal{D}(A) \subset \mathcal{D}(A)$.

- The origin of the closed-loop system (7.11)- (7.14) is

- globally finite-time stable for $\mu<0$ and the settling-time functional $T: \mathbb{H} \rightarrow[0,+\infty)$ admits the estimate

$$
T\left(x_{0}\right) \leq \frac{\left\|x_{0}\right\|_{\mathbf{d}}^{-\mu}}{-\rho \mu}
$$

- globally exponentially stable for $\mu=0$ and

$$
\|x(t)\|_{\mathbf{d}} \leq\left\|x_{0}\right\|_{\mathbf{d}} e^{-\rho t}
$$


- globally nearly fixed-time stable for $\mu>0$ and

$$
\forall r>0 \quad: \quad\|x(t)\|_{\mathbf{d}} \leq r, \quad \forall t \geq \frac{1}{\rho \mu r^{\mu}} .
$$

independently of initial conditions.

- The canonical homogeneous norm $\|\cdot\|_{\mathbf{d}}$ is a Lyapunov function of the closed-loop system (7.11)- (7.14) such that

$$
\frac{d}{d t}\|x(t)\|_{\mathbf{d}} \stackrel{\text { a.e. }}{\leq}-\rho\|x(t)\|_{\mathbf{d}}^{1+\mu}, t>0
$$

for any strong solution of the closed-loop system (7.11)- (7.14).

The condition $\mathcal{D}(A) \subset \mathcal{D}\left(G_{\mathbf{d}}\right)$ in the latter theorem is required for differentiability of the canonical homogeneous on $\mathcal{D}(A)$. If the canonical homogeneous norm in differentiable on whole $\mathbb{H} \backslash\{\mathbf{0}\}$, then the mentioned condition can be omitted.

Corollary 7.8 (Polyakov, 2020, page 279). If a linear dilation $\mathbf{d}$ in $\mathbb{H}=L^{2}\left(\mathbb{R}^{n}, \mathbb{R}^{m}\right)$ is given by $(3.17)$ then the condition $\mathcal{D}(A) \subset$ $\mathcal{D}\left(G_{\mathbf{d}}\right)$ can be omitted in Theorem 7.7.

\subsubsection{Examples of finite-time and fixed-time stabilization of PDEs Homogeneous stabilization of heat equation on $\mathbb{R}^{n}$}

Let $\mathbb{H}=\mathbb{X}=L^{2}\left(\mathbb{R}^{n}, \mathbb{R}\right)$ and $A=\Delta: \mathcal{D}(\Delta) \subset \mathbb{H} \rightarrow \mathbb{H}$ be the Laplace operator and $B=I$ be an identity operator, where $\mathcal{D}(\Delta)=H^{2}\left(\mathbb{R}^{n}, \mathbb{R}\right) \cap$ $H_{0}^{1}\left(\mathbb{R}^{n}, \mathbb{R}\right)$ is the domain of $\Delta$

Let $\mathbf{d}$ be selected in the form (3.17) with $G_{\alpha}=\alpha>-\frac{n}{4}$ and $G_{\beta}=\beta I_{n}$, where $\beta=-\frac{1}{2}$. In this case, from (3.20) we derive have $\|x\|_{\mathbf{d}}=\|x\|^{\frac{4}{4 \alpha+n}}$.

In Example 3.2 we have shown that the Laplace operator is $\mathbf{d}$ homogeneous of the degree $2 \beta=-1$. Taking into account Corollary 7.8 we conclude that Condition B) of Theorem 7.7 holds.

Let us select in $(7.14) K \in \mathcal{L}(\mathbb{H}, \mathbb{X})$ as follows

$$
K x=-\left(\alpha+\frac{n}{4}\right) x, \quad x \in \mathbb{H} .
$$


The generator of the dilation $\mathbf{d}$ is given by (see Lemma 3.18)

$$
\left(G_{\mathbf{d}} x\right)(z)=\alpha z(x)-\frac{1}{2} z \cdot \nabla x(z)
$$

where $z=\left(z_{1}, \ldots, z_{n}\right)^{\top} \in \mathbb{R}^{n}$ and $x \in \mathcal{D}\left(G_{\mathbf{d}}\right)$.

In this case, for any $x \in C_{c}^{\infty}\left(\mathbb{R}^{n}, \mathbb{R}\right)$ and any open bounded connected set $\Omega \subset \mathbb{R}^{n}$ with a smooth boundary, which contains a support of $x$, we have

$$
\begin{gathered}
\left\langle x,\left(A+B K+G_{\mathbf{d}}\right) x\right\rangle= \\
\langle x, \Delta x\rangle+\langle x, B K x\rangle+\alpha\langle x, x\rangle-\frac{1}{2} \int_{\Omega} \sum_{i=1}^{n} z_{i} x(z) \frac{\partial x(z)}{\partial z_{i}} d z= \\
\langle x, \Delta x\rangle-\frac{n}{4}\langle x, x\rangle-\frac{1}{4} \int_{\Omega} \sum_{i=1}^{n} z_{i} \frac{\partial x^{2}(z)}{\partial z_{i}} d z= \\
-\int_{\Omega}|\nabla x(z)|^{2} d z+\frac{n}{4}\langle x, x\rangle-\frac{n}{4} \int_{\Omega} x^{2}(z) d z \leq 0,
\end{gathered}
$$

where the integration by parts (for the first and the third term) has been utilized on the last step. Since $C_{c}^{\infty}\left(\mathbb{R}^{n}, \mathbb{R}\right)$ is dense in $L^{2}$ then Condition C) of Theorem 7.7 is fulfilled.

Finally, selecting the group $\mathbf{d}_{1}(s)=I$ (with the generator $G_{\mathbf{d}_{1}}=\mathbf{0}$ ) we conclude that all conditions of Therem 7.7 are satisfied. This means that the control

$$
u(x)=-\frac{4 \alpha+n}{4} \frac{x}{\|x\|^{\frac{4}{4 \alpha+n}}}
$$

is continuous for $\alpha>1-\frac{n}{4}$, globally bounded for $\alpha=1-\frac{n}{4}$ and stabilizes the heat system

$$
\dot{x}=\Delta x+u(x)
$$

in a finite time $T\left(x_{0}\right) \leq \frac{4\left\|x_{0}\right\|^{\frac{4}{4 \alpha+n}}}{4 \alpha+n}$. For $\alpha=1-\frac{n}{4}$ the control $u$ is discontinuous at the origin, so mild Filippov solutions have to be considered for $t \geq T\left(x_{0}\right)$ in this case.

\section{Homogeneous control for a wave equation on a line}

Let

$$
\mathbb{H}=H^{1}(\mathbb{R}, \mathbb{R}) \times L^{2}(\mathbb{R}, \mathbb{R})
$$


with an inner product to be defined below.

Let us consider a wave equation represented in the form (7.11) using the notations:

$$
A=\left(\begin{array}{cc}
O & I \\
\frac{\partial^{2}}{\partial z^{2}} & O
\end{array}\right): \mathcal{D}(A) \subset \mathbb{H} \rightarrow \mathbb{H},
$$

where

$$
\begin{gathered}
\mathcal{D}(A)=H^{2}(\mathbb{R}, \mathbb{R}) \times H^{1}(\mathbb{R}, \mathbb{R}), \\
O \in \mathcal{L}\left(L^{2}(\mathbb{R}, \mathbb{R}), L^{2}(\mathbb{R}, \mathbb{R})\right) \quad \text { and } \quad I \in \mathcal{L}\left(L^{2}(\mathbb{R}, \mathbb{R}), L^{2}(\mathbb{R}, \mathbb{R})\right)
\end{gathered}
$$

are zero and identity operators, respectively, and

$$
B=\left(\begin{array}{c}
O \\
I
\end{array}\right): L^{2}(\mathbb{R}, \mathbb{R}) \rightarrow \mathbb{H} .
$$

Let the inner product in $\mathbb{H}$ be defined as follows

$$
\langle x, \tilde{x}\rangle=\int_{\mathbb{R}} x^{\top}(z) P \tilde{x}(z)+p_{22} \frac{\partial x_{1}}{\partial z} \frac{\partial \tilde{x}_{1}}{\partial z} d z,
$$

where

$$
P:=\left(\begin{array}{ll}
p_{11} & p_{12} \\
p_{12} & p_{22}
\end{array}\right)=Q^{-1}
$$

with

$$
Q=\left(\begin{array}{ll}
q_{11} & q_{12} \\
q_{12} & q_{22}
\end{array}\right) \in \mathbb{R}^{2 \times 2}
$$

being a positive definite solution of the system of linear matrix inequalities (LMIs) and equations:

$$
\begin{gathered}
\left(\begin{array}{cc}
\nu-1.5 \mu & 1 \\
0 & \nu-0.5 \mu
\end{array}\right) Q+Q\left(\begin{array}{cc}
\nu-1.5 \mu & 0 \\
1 & \nu-0.5 \mu
\end{array}\right)+y^{\top} b^{\top}+b y=0, \\
\left(\begin{array}{cc}
\nu-1.5 \mu & 0 \\
0 & \nu-0.5 \mu
\end{array}\right) Q+Q\left(\begin{array}{cc}
\nu-1.5 \mu & 0 \\
0 & \nu-0.5 \mu
\end{array}\right) \succ 0, \quad Q \succ 0,
\end{gathered}
$$

where $\nu>\max \{0,1.5 \mu\}, b=\left(\begin{array}{ll}0 & 1\end{array}\right)^{\top} \in \mathbb{R}^{2}, y=\left(\begin{array}{ll}y_{1} & y_{2}\end{array}\right) \in \mathbb{R}^{1 \times 2}$.

The operator $A$ is a generator of a strongly continuous semigroup of linear bounded operators on $\mathbb{H}$ (see, e.g., Pazy, 1983, Section 7.4 for more details).

One can be shown that this system of LMIs is always feasible with respect to $Q$ and $y$. Since from (7.19) we conclude

$$
(\nu-1.5 \mu) q_{11}+q_{12}=0
$$


then taking into account $P=Q^{-1} \succ 0$ it is easy to see that

$$
0<(\nu-1.5 \mu) p_{22}=p_{12} .
$$

Let the operator $K: \mathbb{H} \rightarrow L^{1}(\mathbb{R}, \mathbb{R})$ be defined as

$$
K x:=y Q^{-1} x, \quad x \in \mathbb{H},
$$

where the pair $(Q, y)$ is a solution of the LMIs (7.19), (7.20).

A) Let the uniformly continuous semigroup $\mathbf{d}_{1}$ of linear bounded operators on $\mathbb{H}$ be defined as follows

$$
\mathbf{d}_{1}(s) x=\left(\begin{array}{cc}
e^{-\mu s} & 0 \\
0 & 1
\end{array}\right) x .
$$

Its generator

$$
G_{\mathbf{d}_{1}} x=\left(\begin{array}{cc}
-\mu & 0 \\
0 & 0
\end{array}\right) x,
$$

obviously, satisfies the condition A) of Theorem 7.7 with $\gamma_{\min }=$ $\min \{0,-\mu\}$ and $\gamma_{\max }=\max \{0,-\mu\} \geq 0$.

B) Let us introduce the dilation $\mathbf{d}$ on $\mathbb{H}$ as follows

$$
(\mathbf{d}(s) x)(z)=\left(\begin{array}{cc}
e^{s(\nu-\mu)} & 0 \\
0 & e^{s \nu}
\end{array}\right) x\left(e^{\mu s} z\right), \quad x \in \mathbb{H}, \quad z \in \mathbb{R} .
$$

The dilation $\mathbf{d}$ has the generator $G_{\mathbf{d}}: \mathcal{D}\left(G_{\mathbf{d}}\right) \subset \mathbb{H} \rightarrow \mathbb{H}$ defined as

$$
G_{\mathbf{d}} x=\left(\begin{array}{rr}
\nu-\mu & 0 \\
0 & \nu
\end{array}\right) x+\mu z \frac{\partial x}{\partial z},
$$

where $\mathcal{D}\left(G_{\mathbf{d}}\right)=\left\{x \in \mathbb{H}: z \frac{\partial x}{\partial z} \in \mathbb{H}\right\}$.

According to Proposition 3.4 the dilation $\mathbf{d}$ is strictly monotone on $\mathbb{H}$ if there exists $\beta>0$ such that $\left\langle G_{\mathbf{d}} x, x\right\rangle \geq \beta\|x\|^{2}$ for any $x \in$ $C_{c}^{\infty}(\mathbb{R}, \mathbb{R}) \times C_{c}^{\infty}(\mathbb{R}, \mathbb{R})$, where $\|x\|^{2}=\sqrt{\langle x, x\rangle}$. Using integration by parts we derive

$$
\begin{gathered}
\left\langle G_{\mathbf{d}} x, x\right\rangle=\int_{\mathbb{R}} x^{\top}(z) P\left(\left(\begin{array}{rr}
\nu-\mu & 0 \\
0 & \nu
\end{array}\right) x(z)+\mu z \frac{\partial x(s)}{\partial z}\right) d z+ \\
p_{22} \int_{\mathbb{R}}(\nu-\mu)\left(\frac{\partial x_{1}(z)}{\partial z}\right)^{2}+\mu \frac{\partial x_{1}(z)}{\partial z} \frac{\partial}{\partial z}\left(z \frac{\partial x_{1}(z)}{\partial z}\right) d z= \\
\int_{\mathbb{R}} x^{\top}(z) P\left(\begin{array}{rr}
\nu-\mu & 0 \\
0 & \nu
\end{array}\right) x(z) d z+\frac{\mu}{2} \int_{\mathbb{R}} z \frac{\partial}{\partial z}\left(x^{\top}(z) P x(z)\right) d z+
\end{gathered}
$$




$$
\begin{aligned}
& p_{22} \nu \int_{\mathbb{R}}\left(\frac{\partial x_{1}(z)}{\partial z}\right)^{2} d z+\frac{\mu}{2} \int_{\mathbb{R}} z \frac{\partial}{\partial z}\left(\frac{\partial x_{1}(z)}{\partial z}\right)^{2} d z= \\
& \int_{\mathbb{R}} x^{\top}(z) P\left(\begin{array}{cc}
\nu-1.5 \mu & 0 \\
0 & \nu-0.5 \mu
\end{array}\right) x(z)+p_{22}(\nu-0.5 \mu)\left(\frac{\partial x_{1}(z)}{\partial z}\right)^{2} d z \geq \beta\|x\|^{2},
\end{aligned}
$$

where

$$
\beta \geq \min \left\{\lambda_{\min }\left(P^{\frac{1}{2}}\left(\begin{array}{cc}
\nu-\frac{3 \mu}{2} & 0 \\
0 & \nu-\frac{\mu}{2}
\end{array}\right) P^{-\frac{1}{2}}+P^{-\frac{1}{2}}\left(\begin{array}{cc}
\nu-\frac{3 \mu}{2} & 0 \\
0 & \nu-\frac{\mu}{2}
\end{array}\right) P^{\frac{1}{2}}\right), \nu-\frac{\mu}{2}\right\}
$$

The LMI (7.20) implies $\beta>0$. The dilation $\mathbf{d}$ has the form (3.17) and Condition B) of Theorem 7.7 is fulfilled in the view of Corollary 7.8.

C) Given $x=\left(x_{1}, x_{2}\right)^{\top} \in C_{c}^{\infty}(\mathbb{R}, \mathbb{R}) \times C_{c}^{\infty}(\mathbb{R}, \mathbb{R})$ and $A_{0}=\left(\begin{array}{ll}0 & I \\ 0 & 0\end{array}\right)$ we have

$$
\left\langle\left(A+B K+G_{\mathbf{d}}\right) x, x\right\rangle=\left\langle\left(A_{0}+B K+G_{\mathbf{d}}\right) x, x\right\rangle+\left\langle\left(\begin{array}{c}
0 \\
\partial^{2} x_{1} \\
\partial z^{2}
\end{array}\right), x\right\rangle .
$$

Using (7.19) we derive

$$
\left\langle x,\left(A_{0}+B K+G_{\mathbf{d}}\right) x\right\rangle=p_{22} \int_{\mathbb{R}} \frac{\partial x_{1}(z)}{\partial z} \frac{\partial x_{2}(z)}{\partial z} d z+p_{22}(\nu-0.5 \mu) \int_{\mathbb{R}}\left(\frac{\partial x_{1}(z)}{\partial z}\right)^{2} d z .
$$

Using integration by parts we derive

$$
\left\langle x,\left(\begin{array}{c}
\mathbf{0} \\
\frac{\partial^{2} x_{1}}{\partial z^{2}}
\end{array}\right)\right\rangle=\int_{\mathbb{R}}-p_{12}\left(\frac{\partial x_{1}(z)}{\partial z}\right)^{2}-p_{22} \frac{\partial x_{1}(z)}{\partial z} \frac{\partial x_{2}(z)}{\partial z} d z,
$$

Therefore, taking into account the identity $0<(\nu-1.5 \mu) p_{22}=p_{12}$ we conclude

$$
\left\langle x,\left(A+B K+G_{\mathbf{d}}\right) x\right\rangle=\mu p_{22} \int_{\mathbb{R}}\left(\frac{\partial x_{1}(z)}{\partial z}\right) d z .
$$

For $\mu \leq 0$ we, obviously, have $\left\langle x,\left(A+B K+G_{\mathbf{d}}\right) x\right\rangle \leq 0$. On the other hand, if $\nu \geq 2.5 \mu>0$ then using the representation for $\left\langle x, G_{\mathbf{d}} x\right\rangle$ we derive

$$
\left\langle x,\left(A+B K+0.5 G_{\mathbf{d}}\right) x\right\rangle \leq-\frac{p_{22}(\nu-2.5 \mu)}{2} \int_{\mathbb{R}}\left(\frac{\partial x_{1}(z)}{\partial z}\right) d z \leq 0 .
$$

Therefore, the condition C) of Theorem 7.7 holds for any $\mu \in \mathbb{R}$ and any $\nu \geq \max \{0,2.5 \mu\}$.

D) Finally, $\mathbf{d}(s) B K \mathbf{d}_{1}(s)=B K \mathbf{d}(s)$ for any $s \in \mathbb{R}$ and the operator $A$ is $\mathbf{d}$-homogeneous of the degree $\mu$. Indeed,

$$
(A \mathbf{d}(s) x)(z)=A\left(\begin{array}{c}
e^{(\nu-\mu) s} x_{1}\left(e^{\mu s_{z}}\right) \\
e^{\nu s} x_{2}\left(e^{\mu s} z\right)
\end{array}\right)=\left(\begin{array}{c}
e^{\nu s} x_{2}\left(e^{\mu s} z\right) \\
\left.e^{(\nu+\mu) s} \frac{\partial^{2} x_{1}(q)}{\partial q^{2}}\right|_{q=e^{\mu s_{z}}}
\end{array}\right)=e^{\mu s}(\mathbf{d}(s) A x)(z)
$$


and the condition D) of Theorem 7.7 is fulfilled.

Therefore, the homogeneous feedback control of the form (7.14) steers all trajectories of the wave system to

- the origin in a finite time if $\mu<0$;

- a neighborhood of the origin in a fixed time independently of the initial state if $\mu>0$.

In the general case, the homogeneous norm $\|\cdot\|_{\mathbf{d}}$ is defined implicitly, see (3.20). For $\mu=-1, \nu=\frac{1}{2}$ the canonical homogeneous norm can be found as a unique positive solution of the quartic equation

$$
V^{4}=a V^{2}+b V+c
$$

with $V=e^{s_{x}}=\|x\|_{\mathbf{d}}$,

$a=p_{22} \int_{\mathbb{R}} x_{2}^{2}(z)+\left(x_{1}^{\prime}(z)\right)^{2} d z, \quad b=2 p_{12} \int_{\mathbb{R}} x_{1}(z) x_{2}(z) d z, \quad c=p_{11} \int_{\mathbb{R}} x_{1}^{2}(z) d z$.

In this case $\|x\|_{\mathbf{d}}$ can be computed using Ferrari formulas, i.e., the homogeneous finite-time stabilizing feedback (7.14) for the wave equation admits an explicit representation.

\section{Distributed finite-time control of the heat equation on $[0,1]$}

Inspired by Polyakov et al., 2018, let us consider a distributed finite-time control for the following heat system on $[0,1]$ :

$$
\frac{\partial x}{\partial t}=\frac{\partial^{2} x}{\partial z^{2}}+\phi(z) u(t, z), x(t, 0)=x(t, 1)=0, x(0, z)=x_{0}(z),
$$

where $x$ is the system state, $u$ is the distributed control, $\phi:[0,1] \rightarrow \mathbb{R}_{+}$ is a continuous function such that

$$
c z^{2} \leq \phi(z) \quad \text { for } \quad z \in[0,1],
$$

for some $c>0$. The function $\phi$ represents possibly non-uniform feedback gain on $[0,1]$. The case $\phi \equiv 1$ is studied in Pisano et al., 2011.

Let $\langle\cdot, \cdot\rangle$ and $\|\cdot\|=\sqrt{\langle\cdot, \cdot\rangle}$ be, respectively, the inner product and the norm in $L^{2}((0,1), \mathbb{R})$. Let us define the control $u$ law as follows

$$
u=-\frac{c^{-1} x}{\sqrt{\|x\|}} .
$$


Using (7.21) and the integration by parts, for any classical solution of the system we derive

$$
\begin{aligned}
& \frac{1}{2} \frac{d\|x\|^{2}}{d t}=\left\langle x, \frac{\partial^{2} x}{\partial z^{2}}\right\rangle+\langle x, \phi(z) u(x)\rangle= \\
& -\left\|\frac{\partial x}{\partial z}\right\|^{2}-\frac{c^{-1}}{\sqrt{\|x\|}}\langle x, \phi(z) x\rangle \leq-\left\|\frac{\partial x}{\partial z}\right\|^{2}-\frac{1}{\sqrt{\|x\|}}\|z x\|^{2}= \\
& -\left\|\frac{\partial x}{\partial z}\right\|^{2}-2\left\langle\frac{z x}{\sqrt[4]{\|x\|}}, \frac{\partial x}{\partial z}\right\rangle-\left\|\frac{z x}{\sqrt[4]{\|x\|}}\right\|^{2}+2 \frac{1}{\sqrt[4]{\|x\|}}\left\langle z x, \frac{\partial x}{\partial z}\right\rangle= \\
& -\left\|\frac{\partial x}{\partial z}+\frac{1}{\sqrt[4]{\|x\|}} z x\right\|^{2}-\|x\|^{\frac{3}{4}} .
\end{aligned}
$$

Hence, $\|x(t)\|=0$ for $\forall t \geq \frac{4}{5}\left\|x_{0}\right\|^{\frac{5}{4}}$.

\section{Boundary finite-time control of the heat equation on $[0,1]$}

The distributed control of a heat system on $[0,1]$ can designed as a trivial conclusion of Example (7.7). Boundary finite-time stabilization of the heat system is much more complicated problem Coron and Nguyen, 2017, Polyakov et al., 2017, Espitia et al., 2019.

Let us consider the heat system

$$
\frac{\partial v}{\partial t}=\frac{\partial^{2} v}{\partial x^{2}}, \quad v(0)=v_{0}
$$

with boundary control $\zeta$

$$
(v(t))(0)=0 \quad(v(t))(1)=\zeta(t), t>0
$$

where $v(t) \in L^{2}((0,1), \mathbb{R})$.

The control aim is to steer the state $v(t)$ of the system (7.22), (7.23) to zero in a finite time by means of a linear switching feedback control

$$
\zeta(t)=K_{\sigma(t)} v(t)
$$

where $K_{i}: L^{2}((0,1), \mathbb{R}) \rightarrow \mathbb{R}, i \in \mathbb{Z}$ is a family of linear bounded functionals and $\sigma: \mathbb{R}_{+} \rightarrow \mathbb{Z}$ is a state dependent switching function governed by the discrete equation

$$
\sigma(t)=G\left(\sigma\left(t^{-}\right), v\left(t^{-}\right)\right),
$$


with $G: \mathbb{Z} \times L^{2}((0,1), \mathbb{R}) \rightarrow \mathbb{Z}, t^{-}=t+0^{-}$and $\sigma(0) \in \mathbb{Z}$.

The hybrid (switched) linear system (7.22) - (7.25) has two components: $v(t)$ - continuous state and $\sigma(t)$ - discrete state. Therefore, its solution is a tuple $(v, \sigma): v \in C^{0}\left([0, T), L^{2}((0,1), \mathbb{R})\right)$ and $\sigma:(0, T) \rightarrow \mathbb{Z}$ such that $\sigma(t)$ satisfies the discrete equation (7.25) for all $t \in(0, T)$ and $v$ is a solution to the heat system (7.22) - (7.24) understood in the weak sense:

$$
\begin{gathered}
-\int_{0}^{1} v_{0}(x) \xi(0, x) d x-\int_{0}^{T} \int_{0}^{1} v(t, x) \xi_{t}(t, x) d x d t \\
+\int_{0}^{T} K_{\sigma(t)} v(t, \cdot) \xi_{x}(t, 1) d t-\int_{0}^{T} \int_{0}^{1} v(t, x) \xi_{x x}(t, x) d x d t=0
\end{gathered}
$$

for all $\xi \in C^{2}([0, T) \times[0,1])$ with compact support in $[0, T) \times[0,1]$ such that $\xi$ vanishes at $[0, T) \times\{0,1\}$.

We refer reader to Coron and Nguyen, 2017 for more details about existence of solutions and finite-time stabilization of (7.22) - (7.24) in the case of time-dependent switchings. An information about switched systems can be found in Liberzon, 2003.

Given positive number $\lambda>0$ the backstepping approach (see, e.g., Krstic and Smychlyaev, 2008, Coron and Nguyen, 2017 for the details) introduces the boundary control

$$
\zeta(t)=\int_{0}^{1} k(1, y, \lambda) v(t, y) d y
$$

where

$$
k(x, y, \lambda)=-\lambda y \frac{I_{1}\left(\sqrt{\lambda\left(x^{2}-y^{2}\right)}\right)}{\left.\sqrt{\lambda\left(x^{2}-y^{2}\right)}\right)}
$$

and $I_{m}$ with $m \in \mathbb{Z}$ is the modified Bessel function of the first kind (see, e.g., Watson, 1996). If we denote

$$
(F(\lambda) u)(x)=-\int_{0}^{x} k(x, y, \lambda) u(y) d y
$$

then the state transformation $u=v+F(\lambda) v$ applied to the system (7.22), (7.23) yields

$$
\frac{\partial u}{\partial t}=\frac{\partial^{2} u}{\partial x^{2}}-\lambda u, \quad u(t, 0)=0, \quad u(t, 1)=0 .
$$


The inverse transformation is defined as

$$
v=(I+F(-\lambda)) u,
$$

where $u, v \in L^{2}((0,1), \mathbb{R})$. We refer reader to Krstic and Smychlyaev, 2008 and Coron and Nguyen, 2017 for more details about backstepping transformation.

It is easy to see (using the Lyapunov function defined as $V(u)=$ $\|u\|^{2}$ ) that the system (7.28) is asymptotically stable and

$$
\|u(t)\| \leq\|u(0)\| e^{-\lambda t} .
$$

Following Coron and Nguyen, 2017 we also use the backstepping transformation in order to design a finite-time control of the form (7.26) with switching parameter $\lambda$. The system (7.28) will be utilized as a sort of comparison system in order to prove stability and finite-time convergence of solution to zero as well as to estimate the settling time.

One can be shown Coron and Nguyen, 2017 that if $z \in L^{2}((0,1), \mathbb{R})$ then

$$
\begin{gathered}
\|z+F(\lambda) z\| \leq \Psi_{1}(\lambda)\|z\| \text { and }\|z+F(-\lambda) z\| \leq \Psi_{-1}(\lambda)\|z\|, \\
\Psi_{1}(\lambda)=1+\frac{\left.\left.\lambda \sqrt{\int_{0}^{1} \int_{0}^{x} y^{2}\left(I_{0}\left(\sqrt{\lambda\left(x^{2}-y^{2}\right)}\right)-I_{2}\left(\sqrt{\lambda\left(x^{2}-y^{2}\right.}\right)\right.}\right)\right)^{2} d y d x}{2}, \\
\Psi_{-1}(\lambda)=1+\frac{\lambda \sqrt{\iint_{0}^{1} y^{2}\left(J_{0}\left(\sqrt{\lambda\left(x^{2}-y^{2}\right)}\right)+J_{2}\left(\sqrt{\lambda\left(x^{2}-y^{2}\right)}\right)\right)^{2} d y d x}}{2},
\end{gathered}
$$

where $J_{k}$ is the Bessel function of the first kind.

Now let us consider the heat system (7.22), (7.23) with the hybrid boundary feedback control

$$
\xi(t)=\int_{0}^{1} k\left(1, y, 2^{\sigma(t)}\right) v(t, y) d y
$$

where the switching function $\sigma$ is governed by the equation (7.25) with

$$
G(\sigma, v)=\left\{\begin{array}{cll}
i+1 & \text { if } & \sigma=i \text { and }\|v\| \leq r_{i+1} \\
i & \text { if } & \sigma=i \text { and } r_{i+1}<\|v\|<r_{i-1} \\
i-1 & \text { if } & \sigma=i \text { and }\|v\| \geq r_{i-1}
\end{array}\right.
$$


where $r_{0}=1, r_{i}=e^{-q_{i}} r_{i-1}, i \in \mathbb{Z}$ and the numbers $q_{i}$ are defined by formula (7.31).

In Polyakov et al., 2017 it is shown that

$$
q_{i}=\ln \Psi_{1}\left(2^{i}\right)+\ln \Psi_{-1}\left(2^{i}\right),
$$

then $q_{i}>0$ for all $i \in \mathbb{Z}, r_{i} \rightarrow 0$ as $i \rightarrow+\infty$ and

$$
\lim _{i \rightarrow+\infty} \frac{q_{i+1}}{q_{i}}=\sqrt{2}
$$

Moreover, for any initial condition

$$
v(0)=v_{0} \in L^{2}((0,1), \mathbb{R})
$$

and

$$
\sigma(0)=i_{0} \in \mathbb{Z} \quad \text { with } \quad\|v(0)\| \in\left(r_{i_{0}+1}, r_{i_{0}}\right],
$$

the system $(7.22),(7.23),(7.29),(7.25),(7.30)$ has a unique solution $(v, \sigma): v \in C^{0}\left([0, T), L^{2}((0,1), \mathbb{R})\right)$ and $\sigma:(0, T) \rightarrow \mathbb{Z}$ such that

$$
\|v(t)\| \rightarrow 0 \text { as } t \rightarrow T^{-},
$$

where $T=T\left(u_{0}\right)$ satisfies

$$
T \leq \sum_{i=i_{0}}^{+\infty} \frac{q_{i}+q_{i+1}}{2^{i}}<+\infty .
$$

Examples of the prescribed-time (i.e., $T\left(u_{0}\right) \equiv$ const) stabilization of PDEs can be found in Espitia et al., 2019, Steeves et al., 2020. 
Appendices 


\section{A}

\section{Notation}

- $\mathbb{N}$ is the set of natural numbers; $\mathbb{Z}$ is the set of integers; $\mathbb{R}$ is the set of reals; $\overline{\mathbb{R}}=\mathbb{R} \cup\{-\infty\} \cup\{+\infty\}$ and $\mathbb{R}_{+}=[0,+\infty) ; \mathbb{C}$ is the set of complex numbers.

- $A \times B$ denotes the Cartesian product of sets $A$ and $B$.

- The inner product of vectors $x=\left(x_{1}, \ldots, x_{n}\right)$ and $y=\left(y_{1}, \ldots, y_{n}\right)$ from a $n$-dimensional Euclidean space is given by $x \cdot y=\sum_{i=1}^{n} x_{i} y_{i}$, where $x_{i}$ and $y_{i}$ are coordinates of the vectors $x$ and $y$ in an orthonormal basis.

- $\mathbb{B}$ is a real Banach space with a norm $\|\cdot\|$ and $\mathbb{H}$ is a real Hilbert space with an inner product $\langle\cdot, \cdot\rangle$.

- $\mathbf{0} \in \mathbb{B}$ denotes a zero element a Banach space.

- $\operatorname{span}\left\{e_{1}, e_{2}, \ldots, e_{k}\right\}:=\left\{\alpha_{1} e_{1}+\ldots+\alpha_{k} e_{k}: \alpha_{i} \in \mathbb{R}, i=1,2, \ldots, k\right\}$, where $e_{i} \in \mathbb{B}$.

- $S=\{x \in \mathbb{B}:\|x\|=1\}$ is the unit sphere in $\mathbb{B}$.

- The notation $\|\cdot\|_{X}$ is utilized if it is necessary to indicate that this is a norm in a space $X$.

- $\mathcal{L}(X, Y)$ is the space of linear bounded operators $X \rightarrow Y$, where $X$ and $Y$ are Banach spaces, and

$$
\|A\|_{\mathcal{L}(X, Y)}=\sup _{u \neq 0} \frac{\|A u\|_{Y}}{\|u\|_{X}}, \quad\lfloor A\rfloor_{\mathcal{L}(X, Y)}=\inf _{u \neq 0} \frac{\|A u\|_{Y}}{\|u\|_{X}}, \quad A \in \mathcal{L}(X, Y) .
$$


We also use the notations $\|A\|$ and $\lfloor A\rfloor$ for shortness if a context is clear.

- $f_{1}\left(f_{2}\right)$ and $f_{1} \circ f_{2}$ denote the composition of nonlinear operators (functions) $f_{1}$ and $f_{2}$. In the case of linear operators $A$ and $B$, for simplicity, the brackets and the sign " o" can be omitted, i.e., $A B$ denotes the composition of linear operators $A$ and $B$.

- If $P=P^{\top} \in \mathbb{R}^{n \times n}$ then $P \succ 0$ (resp. $\succeq 0$ ) means that the matrix $P$ is positive definite (resp. semidefinite) and $P \prec 0$ (resp. $\preceq 0$ ) means that the matrix $P$ is negative definite (resp. semidefinite).

- $\lambda_{\min }(P)$ and $\lambda_{\max }(P)$ denotes minimum and maximum eigenvalues of a symmetric matrix $P=P^{\top} \in \mathbb{R}^{n}$.

- $\operatorname{rank}(A)$ denotes the rank of $A \in \mathbb{R}^{m \times n}$.

- $\operatorname{tr}(A)$ denotes the trace of $A \in \mathbb{R}^{n \times n}$.

- $G_{\mathbf{d}}^{-\top}:=\left(G_{\mathbf{d}}^{-1}\right)^{\top}$.

- $I \in \mathcal{L}(\mathbb{B}, \mathbb{B})$ denotes the identity operator in $\mathbb{B}$ and $I_{n}$ is the identity matrix in $\mathbb{R}^{n \times n}$.

- $\operatorname{div}(u)=\sum_{i=1}^{n} \frac{\partial u_{i}}{\partial z_{i}}$ for a function $u: \mathbb{R}^{n} \rightarrow \mathbb{R}^{n}$

- $\nabla:=\left(\frac{\partial}{\partial x_{1}}, \ldots, \frac{\partial}{\partial x_{n}}\right)$, and $\Delta:=\nabla \cdot \nabla=\operatorname{div}(\nabla)=\sum_{i=1}^{n} \frac{\partial^{2}}{\partial x_{i}^{2}}$ - Laplace operator.

- $\bar{X}$ denotes the closure of the set $X$ of a metric space.

- $C(X, Y)$ is the space of uniformly continuous functions $X \rightarrow Y$ with the supremum norm, $\|f\|=\sup _{x \in X}\|f(x)\|_{Y}$, where $f \in$ $C(X, Y)$ and $X, Y$ are normed vector spaces.

- $C_{c}^{\infty}\left(\Omega, \mathbb{R}^{m}\right)$ is the space of infinitely differentiable (smooth) functions having compact support in $\Omega$, where $\Omega \subset \mathbb{R}^{m}$ is an open set. 
- $C_{0}^{\infty}\left(\Omega, \mathbb{R}^{m}\right)$ is the space of infinitely differentiable (smooth) functions vanishing on the boundary of $\Omega$.

- Let $L^{p}\left(\Omega, \mathbb{R}^{m}\right)$ denotes the Banach space of functions $\Omega \rightarrow \mathbb{R}^{m}$

$$
\begin{gathered}
L^{p}\left(\Omega, \mathbb{R}^{m}\right):=\left\{u:\|u\|_{p}<+\infty\right\}, \\
\|u\|_{L^{p}}:=\left(\int_{\Omega}|u(x)|^{p} d x\right)^{1 / p}, \quad 1 \leq p<\infty \\
\|u\|_{L^{\infty}}:=\operatorname{ess} \sup |u(x)|, \quad p=\infty
\end{gathered}
$$

- $L^{2}\left(\Omega, \mathbb{R}^{m}\right)$ is a Hilbert space with the inner product

$$
\langle u, v\rangle_{L^{2}}=\int_{\Omega} u(x) \cdot v(x) d x .
$$

- The Sobolev space $H^{p}\left(\Omega, \mathbb{R}^{m}\right)$ is a Hilbert space with the inner product

$$
\langle u, v\rangle_{H^{p}}:=\sum_{i=0}^{p}\left\langle\nabla^{i} u, \nabla^{i} v\right\rangle_{L^{2}}
$$

and the norm $\|\cdot\|_{H^{p}}=\sqrt{\langle\cdot, \cdot\rangle_{H^{p}}}$.

- $H_{0}^{p}\left(\Omega, \mathbb{R}^{m}\right)$ is the completion of $C_{c}^{\infty}\left(\Omega, \mathbb{R}^{m}\right)$ with respect to $\|\cdot\|_{H^{p}}$.

- If $\Omega_{1} \subset \mathbb{B}$ and $\Omega_{2} \subset \mathbb{B}$ then, by definition, the identity $\Omega_{1}=\Omega_{2}$ means $\Omega_{1} \subset \Omega_{2}$ and $\Omega_{2} \subset \Omega_{1}$.

- $B(r):=\{x \in \mathbb{B}:\|x\|<r\}$ is the open ball in $\mathbb{B}$ of the radius $r \in \mathbb{R}_{+}$with the center at the origin and $B(y, r)=y+B(\varepsilon)$ is an open ball of the radius $\varepsilon>0$ centered at $y \in \mathbb{B}$.

- $\partial \Omega$ is the boundary of a set $\Omega \subset \mathbb{R}^{n}$.

- $\operatorname{int}(\Omega)$ denotes the interior of a set $\Omega \subset \mathbb{R}^{n}$, i.e., $x \in \operatorname{int}(\Omega)$ if and only if $\exists r \in \mathbb{R}_{+}: x+\mathbb{B}(r) \subset \Omega$.

- The set consisting of elements $x_{1}, x_{2}, \ldots, x_{n}$ is denoted $\left\{x_{1}, x_{2}, \ldots, x_{n}\right\}$.

- The power set (i.e., the set of all subsets) of a set $M \subset \mathbb{R}^{n}$ is $2^{M}$. 
- The sign function is defined by

$$
\operatorname{sign}_{\sigma}(\rho):=\left\{\begin{array}{ccc}
1 & \text { if } & \rho>0 \\
-1 & \text { if } & \rho<0 \\
\sigma & \text { if } & \rho=0
\end{array}\right.
$$

where $\sigma \in \mathbb{R}:-1 \leq \sigma \leq 1$. If a concrete value of $\sigma$ is not important for considerations, we use the notation $\operatorname{sign}[\rho]$.

- $\left\lfloor\left. x\right|^{\alpha}:=|x|^{\alpha} \operatorname{sign}[x]\right.$ is the power operation, which preserves the sign of the number $x \in \mathbb{R}$. For example, $\lfloor-2\rceil^{2}=-4$ and $\lfloor 2\rceil^{2}=4$.

- a function $\sigma \in C\left(\mathbb{R}_{+}, \mathbb{R}_{+}\right)$belongs to the class $\mathcal{K}$ if $\sigma(0)=0$ and $\sigma$ is increasing, i.e., $t_{1} \leq t_{2} \Rightarrow \sigma\left(t_{1}\right) \leq \sigma\left(t_{2}\right)$.

- a function $\sigma \in \mathcal{K}$ belongs to the class $\mathcal{K}^{\infty}$ if $\sigma(t) \rightarrow+\infty$ as $t \rightarrow+\infty$.

- a continuous function $\xi: \mathbb{R}_{+} \times \mathbb{R}_{+} \rightarrow \mathbb{R}_{+}$belongs to the class $\mathcal{K} \mathcal{L}$ if $\xi(\cdot, t) \in \mathcal{K}$ for any fixed $t \geq 0$ and $\xi(s, \cdot): \mathbb{R}_{+} \rightarrow \mathbb{R}_{+}$is decreasing to zero for any fixed $s \geq 0$.

- $\mathcal{H}_{\mathbf{d}}(\mathbb{B})$ is a set of $\mathbf{d}$-homogeneous functionals $\mathbb{B} \rightarrow \mathbb{R}$ and $\operatorname{deg}_{\mathbf{d}}(h) \in$ $\mathbb{R}$ is a homogeneity degree of $h \in \mathcal{H}_{\mathbf{d}}(\mathbb{B})$.

- $\mathcal{F}_{\mathbf{d}}(\mathbb{B})$ is a set of $\mathbf{d}$-homogeneous functionals $\mathbb{B} \rightarrow \mathbb{R}$ and $\operatorname{deg}_{\mathbf{d}}(f) \in$ $\mathbb{R}$ is a homogeneity degree of $f \in \mathcal{F}_{\mathbf{d}}(\mathbb{B})$. 


\section{References}

Acary, V. and B. Brogliato. (2010). "Implicit Euler numerical simulations of sliding mode systems". System and Control Letters. 59(5): 284293.

Acary, V., B. Brogliato, and Y. Orlov. (2012). "Chattering-free digital sliding-mode control with state observer and disturbance rejection". IEEE Transactions on Automatic Control. 57(5): 1087-1101.

Aleksandrov, A. and A. Zhabko. (2012). "On the asymptotic stability of solutions of nonlinear systems with delay". Siberian Mathematical Journal. 53(3): 393-403.

Aleksandrov, A., A. Kosov, and A. Platonov. (2012). "On the asymptotic stability of switched homogeneous systems". Systems \& Control Letters. 61(1): 127-133 127-133 127-133.

Allen, M. and E. Isaacson. (1998). Numerical Analysis for Applied Science. A Wiley-Interscience publication. Wiley. ISBN: 9780471552666.

Andreini, A., A. Bacciotti, and G. Stefani. (1988). "Global stabilizability of homogenenous vector fields of odd degree". Systems 8 Control Letters. 10: 251-256.

Andrieu, V., L. Praly, and A. Astolfi. (2008). "Homogeneous Approximation, Recursive Observer Design, and Output Feedback". SIAM Journal of Control and Optimization. 47(4): 1814-1850.

Armstrong-Helouvry, B. (1991). Control of machines with friction. Springer. 
Bacciotti, A. and L. Rosier. (2001). Lyapunov Functions and Stability in Control Theory. Springer.

Bernuau, E., D. Efimov, and W. Perruquetti. (2014a). "Robustness of homogeneous and locally homogeneous differential inclusions". In: European Control Conference.

Bernuau, E., D. Efimov, W. Perruquetti, and A. Polyakov. (2014b). "On homogeneity and its application in sliding mode control". Journal of The Franklin Institute. 351(4): 1866-1901.

Bernuau, E., A. Polyakov, D. Efimov, and W. Perruquetti. (2013). "Verification of ISS, iISS and IOSS properties applying weighted homogeneity". System \& Control Letters. 62(12): 1159-1167.

Bernuau, E. (2013). "Robustness and stability of nonlinear systems: a homogeneous point of view". Theses. Ecole Centrale de Lille.

Bhat, S. P. and D. S. Bernstein. (2005). "Geometric homogeneity with applications to finite-time stability". Mathematics of Control, Signals and Systems. 17: 101-127.

Bhat, S. and D. Bernstein. (2000). "Finite Time Stability of Continuous Autonomous Systems". SIAM J. Control Optim. 38(3): 751-766.

Boyd, S., E. Ghaoui, E. Feron, and V. Balakrishnan. (1994). Linear Matrix Inequalities in System and Control Theory. Philadelphia: SIAM.

Braidiz, Y., A. Polyakov, D. Efimov, and W. Perruquetti. (2019). "Robust finite-time stability of homogeneous systems with respect to multiplicative disturbances". In: Proc. European Control Conference. Naples.

Braidiz, Y., A. Polyakov, D. Efimov, and W. Perruquetti. (2020). "On finite-time stability of sub-homogeneous differential inclusions". In: Proc. 21rst IFAC World Congress. Berlin.

Brogliato, B. and A. Polyakov. (2015). "Globally stable implicit Euler time-discretization of a nonlinear single-input sliding-mode control system". In: IEEE CDC. 5426-5431.

Butcher, J. C. (2008). Numerical Methods for Ordinary Differential Equations. 2nd. New York: John Wiley \& Sons.

Chernous'ko, F. L., I. M. Ananievski, and S. A. Reshmin. (2008). Control of Nonlinear Dynamical Systems. Springer. 
Clarke, F. H., Y. S. Ledyaev, and R. Stern. (1998). "Asymptotic Stability and Smooth Lyapunov Functions". Journal of differential Equations. 149: 69-114.

Coron, J.-M. and H.-M. Nguyen. (2017). "Null controllability and finite time stabilization for the heat equations with variable coefficients in space in one dimension via backstepping approach". Archive for Rational Mechanics and Analysis.

Coron, J.-M. and L. Praly. (1991). "Adding an integrator for the stabilization problem". Systems \& Control Letters. 17(2): 89-104.

Cruz-Zavala, E., J. A. Moreno, and L. M. Fridman. (2011). "Uniform robust exact differentiator". IEEE Trans. Automat. Control. 56(11): 2727-2733. ISSN: 0018-9286. DOI: 10.1109/TAC.2011.2160030. URL: https://mathscinet.ams.org/mathscinet-getitem?mr=2884018.

Curtain, R. and H. Zwart. (1995). An Introduction to Infinite-Dimensional Linear Systems Theory. Springer Verlag.

Dahlquist, G. and A. Björck. (2008). Numerical methods in scientific computing. SIAM.

Dahlquist, G. G. (1963). "A special stability problem for linear multistep methods". BIT Numerical Mathematics. 3(1): 27-43. DOI: 10.1007/ BF01963532.

Dashkovskiy, S., D. Efimov, and E. Sontag. (2011). "Input to state stability and allied system properties". Automation and Remote Control. 72(8): 1579-1614.

Driver, B. (2003). Analysis Tools with Applications. Springer.

Efimov, D. and E. Fridman. (2020). "Converse Lyapunov-Krasovskii theorem for ISS of neutralsystems in Sobolev spaces". Automatica. 118(8): 109042.

Efimov, D. and W. Perruquetti. (2011). "Homogeneity for time-delay systems". In: Proceedings of the 18th World Congress The International Federation of Automatic Control. 3861-3866.

Efimov, D. and W. Perruquetti. (2016). "Conditions of Oscillations and Multi-Homogeneity". Mathematics of Control, Signals, and Systems. 28(3): 1-37.

Efimov, D., W. Perruquetti, and J.-P. Richard. (2014a). "Development of homogeneity concept for time-delay systems". SIAM J. Control Optim. 52(3): 1403-1808. 
Efimov, D., A. Polyakov, E. Fridman, W. Perruquetti, and J.-P. Richard. (2014b). "Comments on Finite-time Stability of Time-Delay Systems". Automatica. 50(7): 1944-1947.

Efimov, D., A. Polyakov, A. Levant, and W. Perruquetti. (2017). "Realization and Discretization of Asymptotically Stable Homogeneous Systems". IEEE Transactions on Automatic Control. 62(11): 59625969.

Efimov, D., A. Polyakov, W. Perruquetti, and J.-P. Richard. (2016). "Weighted Homogeneity for Time-Delay Systems: Finite-Time and Independent of Delay Stability". IEEE Trans. Automatic Control. 61(1): 1-6.

Efimov, D. and A. Aleksandrov. (2020). "On estimation of rates of convergence in Lyapunov-Razumikhin approach". Automatica. 116: 108928. DOI: https://doi.org/10.1016/j.automatica.2020.108928.

Efimov, D., A. Polyakov, and A. Aleksandrov. (2019). "Discretization of homogeneous systems using Euler method with a state-dependent step". Automatica. 109: 108546. DOI: https://doi.org/10.1016/j. automatica.2019.108546.

Engel, K.-J. and R. Nagel. (2000). One-Parameter Semigroups for Linear Evolution Equationsarameter. Springer Verlag: Berlin, Heidelberg, New York.

Erugin, N. (1951). "On the continuouation of solutions of differential equations (in Russian". Prikl. Mat. Mekh. 17(4).

Espitia, N., A. Polyakov, D. Efimov, and W. Perruquetti. (2019). "Boundary time-varying feedbacks for fixed-time stabilization of constant-parameter reaction-diffusion systems". Automatica. 103(5): 398-407.

Falkovich, G. (2011). Fluid Mechanics (A short course for physicists). Cambridge University Press.

Feldbaum, A. (1953). "Optimal Processes in Systems of Automatic Control". Avtom. Telemekh. 14(6): 721-728.

Filippov, A. F. (1988). Differential Equations with Discontinuous Righthand Sides. Kluwer Academic Publishers.

Fischer, V. and M. Ruzhanky. (2016). Quantization on Nilpotent Lie Groups. Springer. 
Folland, G. (1975). "Subelliptic estimates and function spaces on nilpotent Lie groups". Arkiv for Matematik. 13(1-2): 161-207.

Fridman, E. (2014). Introduction to Time-Delay Systems: Analysis and Control. Springer.

Fuller, A. (1960). "Relay control systems optimized for various performance criteria". In: In Proceedings of the 1st IFAC World Congress. $510-519$.

Grune, L. (2000). "Homogeneous state feedback stabilization of homogeneous systems". SIAM Journal of Control and Optimization. 38(4): $1288-1308$.

Haimo, V. (1986). "Finite Time Controllers". SIAM Journal of Control and Optimization. 24(4): 760-770.

Halanay, A. (1966). Differential equations: Stability, oscillations, time lags. Vol. 23. Academic Press.

Hermes, H. (1991). "Nilpotent and high-order approximations of vector field systems". SIAM review. 33(2): 238-264.

Holloway, J. and M. Krstic. (2019). "Prescribed-Time Observers for Linear Systems in Observer Canonical Form". IEEE Transactions on Automatic Control. 64(9): 3905-3912.

Hong, Y., Z. Jiang, and G. Feng. (2010). "Finite-Time Input-to-State Stability and Applications to Finite-Time Control Design". SIAM Journal on Control and Optimization. 48(7): 4395-4418. DOI: 10. 1137/070712043. eprint: http://epubs.siam.org/doi/pdf/10.1137/ 070712043. URL: http://epubs.siam.org/doi/abs/10.1137/070712043. Huang, X., W. Lin, and B. Yang. (2005). "Global finite-time stabilization of a class of uncertain nonlinear systems". Automatica. 41(5): 881888. DOI: https://doi.org/10.1016/j.automatica.2004.11.036.

Huber, O., V. Acary, and B. Brogliato. (2016). "Lyapunov stability and performance analysis of the implicit discrete sliding mode control". IEEE Transactions on Automatic Control. 61(10): 3016-3030.

Husch, L. (1970). "Topological Characterization Of The Dilation and The Translation In Frechet Spaces". Mathematical Annals. 190: 1-5. Isidori, A. (1995). Nonlinear control systems. Third. Communications and Control Engineering Series. Springer-Verlag, Berlin. xvi+549. ISBN: 3-540-19916-0. DOI: 10.1007/978-1-84628-615-5. URL: https: //mathscinet.ams.org/mathscinet-getitem? $\mathrm{mr}=1410988$. 
Izmailov, R. (1987). "The peak effect in stationary linear systems with scalar inputs and outputs". Automation and Remote Control. 48: $1018-1024$.

Jiang, Z.-P. and Y. Wang. (2001). "Input-to-state stability for discretetime nonlinear systems". Automatica. 37: 857-869.

Karafyllis, I. (2006). "Finite-Time Global Stabilization by Means of Time-Varying Distributed Delay Feedback". SIAM Journal on Control and Optimization. 45(1): 320-342. DOI: 10.1137/040616383.

Kawski, M. (1989). "Stabilization of nonlinear systems in the plane". Systems and Control Letter. 12: 169-175.

Kawski, M. (1990). "Homogeneous stabilizing feedback laws". Control Theory and Advanced Technology. 6(4): 497-516.

Kawski, M. (1991). "Families of dilations and asymptotic stability". Analysis of Controlled Dynamical Systems: 285-294.

Khalil, H. K. (2002). Nonlinear Systems. third. Prentice Hall.

Khomenuk, V. V. (1961). "On systems of ordinary differential equations with generalized homogenous right-hand sides". Izvestia vuzov. Mathematica (in Russian). 3(22): 157-164.

Kolmanovskii, V. and A. Myshkis. (1999). Introduction to the Theory and Applications of Functional Differential Equations. Vol. 463. Mathematics and Its Applications. Springer.

Kolmanovsky, V. and V. Nosov. (1986). Stability of functional differential equations. San Diego: Academic.

Korobov, V. (1979). "A solution of the synthesis problem using controlability function". Doklady Academii Nauk SSSR. 248: 1051-1063.

Korobov, V. (1980). "A general approach to the solution of the bounded control synthesis problem in a controllability problem". Math. USSRSb. 37(4): 535-557.

Krasovskii, N. N. (1963). Stability of motion. Applications of Lyapunov's second method to differential systems and equations with delay. Translated by J. L. Brenner. Stanford University Press, Stanford, Calif. vi+188. URL: https://mathscinet.ams.org/mathscinetgetitem? $\mathrm{mr}=0147744$.

Krstic, M. and A. Smychlyaev. (2008). Boundary Control of PDEs. SIAM. 
Levant, A. (2005). "Homogeneity approach to high-order sliding mode design". Automatica. 41(5): 823-830.

Levant, A. (2013). "On fixed and finite time stability in sliding mode control". In: IEEE 52nd Annual Conference on Decision and Control $(C D C)$. 4260-4265. DOI: 10.1109/CDC.2013.6760544.

Levant, A., D. Efimov, A. Polyakov, and W. Perruquetti. (2016). "Stability and Robustness of Homogeneous Differential Inclusions". In: Proc. IEEE CDC.

Liberzon, D. (2003). Switchings in Systems and Control. Birkhauser, Boston.

Lopez-Ramirez, F., A. Polyakov, D. Efimov, and W. Perruquetti. (2018). "Finite-time and fixed-time observer design: Implicit Lyapunov function approach". Automatica. 87(1): 52-60.

Lopez-Ramirez, F., D. Efimov, A. Polyakov, and W. Perruquetti. (2019). "Conditions for fixed-time stability and stabilization of continuous autonomous systems". Systems \& Control Letters. 129: 2635. URL: http: / / www . sciencedirect. com / science / article / pii / S0167691119300787.

Lopez-Ramirez, F., D. Efimov, A. Polyakov, and W. Perruquetti. (2020). "Finite-Time and Fixed-Time Input-to-State Stability: Explicit and ImplicitApproaches". Systems \& Control Letters. 130.

Lyapunov, A. M. (1992). The general problem of the stability of motion. Taylor \& Francis.

Miranda-Villatoro, F., B. Brogliato, and F. Castanos. (2017). "Multivalued Robust Tracking Control of Lagrange Systems: Continuous and Discrete-Time Algorithms". IEEE Transactions on Automatic Control. 62(9): 4436-4450.

Miranda-Villatoro, F., B. Brogliato, and F. Castanos. (2018). "SetValued Sliding-Mode Control of Uncertain Linear Systems: Continuous and Discrete-Time Analysis". SIAM Journal of Control and Optimization. 56(3): 1756-1793.

Misrikhanov, M. and V. Ryabchenko. (2011). "Pole placement for controlling a large scale power system". Automation and Remote Control. 72(10): 2123-2146. 
Moulay, E., M. Dambrine, N. Yeganefar, and W. Perruquetti. (2008). "Finite-time stability and stabilization of time-delay systems". Systems $\&$ Control Letters. 57(7): 561-566. DOI: https://doi.org/10. 1016/j.sysconle.2007.12.002.

Moulay, E. and W. Perruquetti. (2008). "Finite time stability conditions for non-autonomous continuous systems". Internat. J. Control. 81(5): 797-803. ISSN: 0020-7179. DOI: 10.1080/00207170701650303. URL: https://mathscinet.ams.org/mathscinet-getitem? $m r=2406886$.

Moulay, E. and W. Perruquetti. (2006). "Finite time stability and stabilization of a class of continuous systems". J. Math. Anal. Appl. 323(2): 1430-1443. ISSN: 0022-247X. DOI: 10.1016/j.jmaa.2005.11.046. URL: https://mathscinet.ams.org/mathscinet-getitem? $\mathrm{mr}=2260193$.

Myshkis, A. (1995). "Razumikhin's method in the qualitative theory of processes with delay". J. Applied Mathematics and Stochastic Analysis. 8(3): 233-247.

Nakamura, H., Y. Yamashita, and H. Nishitani. (2002). "Smooth Lyapunov functions for homogeneous differential inclusions". In: Proceedings of the 41st SICE Annual Conference. 1974-1979.

Nekhoroshikh, A., D. Efimov, A. Polyakov, W. Perruquetti, and I. Furtat. (2020). "On finite-time stabilization of a class of nonlinear time-delay systems: Implicit Lyapunov-Razumikhin approach". In: Proc. 59th IEEE CDC - Conference on Decision and Control. Jeju Island, South Korea.

Netuka, I. (2011). "The Change-of-Variables Theorem for the Lebesgue Integral". ACTA UNIVERSITATIS MATTHIAE BELII, series MATHEMATICS. 19: 37-42.

Orlov, Y. (2005). "Finite Time Stability and Robust Control Synthesis of Uncertain Switched Systems". SIAM Journal of Control and Optimization. 43(4): 1253-1271.

Pazy, A. (1983). Semigroups of Linear Operators and Applications to Partial Differential Equations. Springer.

Pepe, P. and I. Karafyllis. (2013). "Converse Lyapunov-Krasovskii theorems for systems described by neutral functional differential equations in Hale's form". International Journal of Control. 86(2): $232-243$. 
Pepe, P., I. Karafyllis, and Z.-P. Jiang. (2017). "Lyapunov-Krasovskii characterization of the input-to-state stability for neutral systems in Hale's form". Systems \& Control Letters. 102: 48-56. DOI: https: //doi.org/10.1016/j.sysconle.2017.01.008.

Perrollaz, V. and L. Rosier. (2014). "Finite-time Stabilization of 2x2 Hyperbolic Systems on Tree-shaped Networks". SIAM Journal of Control and Optimization. 52(1): 143-163.

Perruquetti, W., T. Floquet, and E. Moulay. (2008). "Finite-time observers: application to secure communication". IEEE Transactions on Automatic Control. 53(1): 356-360.

Pisano, A., Y. Orlov, and E. Usai. (2011). "Tracking Control of the Uncertain Heat and Wave Equation via Power-Fractional and SlidingMode Techniques". SIAM Journal on Control and Optimization. 49(2): 363-382.

Polyak, B. and G. Smirnov. (2016). "Large deviations for non-zero initial conditions in linear systems". Automatica. 74: 297-307.

Polyakov, A. (2012). "Nonlinear feedback design for fixed-time stabilization of linear control systems". IEEE Transactions on Automatic Control. 57(8): 2106-2110.

Polyakov, A. (2018). "Sliding Mode Control Design Using Canonical Homogeneous Norm". International Journal of Robust and Nonlinear Control. 29(3): 682-701. DOI: 10.1002/rnc.4058.

Polyakov, A. (2019). "Characterization of Finite/Fixed-time Stability of Evolution Inclusions". In: Conference on Decision and Control.

Polyakov, A. (2020). Generalized Homogeneity in Systems and Control. Springer.

Polyakov, A. (2021a). "Homogeneous Lyapunov Functions for Homogeneous Infinite Dimensional Systems with Unbounded Nonlinear Operators". Systems \& Control Letters. 148(2): 104854. URL: https: //hal.inria.fr/hal-02921426.

Polyakov, A. (2021b). "Input-to-State Stability of Homogeneous Infinite Dimensional Systems with Locally Lipschitz Nonlinearities". Automatica. 129: 109615.

Polyakov, A., J.-M. Coron, and L. Rosier. (2017). "On Boundary FiniteTime Feedback Control for Heat Equation". In: 20th IFAC World Congress. 
Polyakov, A., J.-M. Coron, and L. Rosier. (2018). "On Homogeneous Finite-Time Control for Linear Evolution Equation in Hilbert Space". IEEE Transactions on Automatic Control. 63(9): 3143-3150.

Polyakov, A., D. Efimov, and B. Brogliato. (2019). "Consistent Discretization of Finite-time and Fixed-time Stable Systems". SIAM Journal of Control and Optimization. 57(1): 78-103.

Polyakov, A., D. Efimov, E. Fridman, and W. Perruquetti. (2016a). "On homogeneous distributed parameters equations". IEEE Transactions on Automatic Control. 61(11): 3657-3662.

Polyakov, A., D. Efimov, and W. Perruquetti. (2015a). "Finite-time and fixed-time stabilization: Implicit Lyapunov function approach". Automatica. 51(1): 332-340.

Polyakov, A., D. Efimov, and W. Perruquetti. (2016b). "Robust stabilization of MIMO systems in finite/fixed time". International Journal of Robust and Nonlinear Control. 26(1): 69-90.

Polyakov, A., D. Efimov, W. Perruquetti, and J. Richard. (2015b). "Implicit Lyapunov-Krasovski Functionals for Stability Analysis and Control Design of Time-Delay Systems". IEEE Transactions on Automatic Control. 60(12): 3344-3349. DOI: 10.1109/TAC.2015. 2422451.

Qian, C. and J. Li. (2005). "Global finite-time stabilization by output feedback for planar systems without observable linearization". IEEE Transactions on Automatic Control. 50(6): 885-890. DOI: 10.1109/ TAC.2005.849253.

Ríos, H., D. Efimov, J. A. Moreno, W. Perruquetti, and J. G. RuedaEscobedo. (2017). "Time-Varying Parameter Identification Algorithms: Finite and Fixed-Time Convergence". IEEE Transactions on Automatic Control. 62(7): 3671-3678. DOI: 10.1109/TAC.2017. 2673413.

Ríos, H., D. Efimov, A. Polyakov, and W. Perruquetti. (2016). "Homogeneous Time-Varying Systems: Robustness Analysis". IEEE Transactions on Automatic Control. 61(12): 4075-4080. DOI: 10. 1109/TAC.2016.2540806.

Rosier, L. (1992). "Homogeneous Lyapunov Function for Homogeneous Continuous Vector Field". Systems \& Control Letters. 19: 467-473. 
Rosier, L. (1993). "Etude de quelques Problèmes de Stabilization". PhD Thesis, Ecole Normale Superieure de Cachan (France).

Roxin, E. (1966). "On Finite Stability in Control Systems". Rendiconti del Circolo Matematico di Palermo. 15: 273-283.

Ryan, E. (1995). "Universal stabilization of a class of nonlinear systems with homogeneous vector fields". Systems $\&$ Control Letters. 26: 177-184.

Sanchez, T., D. Efimov, and A. Polyakov. (2020). "Discrete-Time Homogeneity: Robustness and Approximation". Automatica.

Sanchez, T., D. Efimov, A. Polyakov, J. Moreno, and W. Perruquetti. (2019). "On homogeneity of discrete-time systems: stability and convergence rates". International Journal of Robust and Nonlinear Control. 29(8): 2406-2421.

Sepulchre, R. and D. Aeyels. (1996a). "Homogeneous Lyapunov Functions and Necessary Conditions for Stabilization". Mathematics of Control, Signals and Systems. 9: 34-58.

Sepulchre, R. and D. Aeyels. (1996b). "Stabilizability does not imply homogeneous stabilizability for controllable homogeneous systems". SIAM Journal of Control and Optimization. 34(5): 1798-1813.

Shtessel, Y., C. Edwards, L. Fridman, and A. Levant. (2014). Sliding Mode Control and Observation. Birkhauser.

Song, Y., Y. Wang, J. Holloway, and M. Krstic. (2017). "Time-varying feedbackfor regulation of normal-form nonlinear systems in prescribed finite time". Automatica. 83: 243-251.

Sontag, E. (1989). "A 'Universal' Construction of Artstein's Theorem on Nonlinear Stabilization". Systems \& Control Letters. 13: 117-123.

Sontag, E. (2007). "Input to state stability: Basic concepts and results". In: Nonlinear and Optimal Control Theory. Ed. by P. P. Nistri and G. Stefani. Springer-Verlag, Berlin. 163-220.

Steeves, D., M. Krstic, and R. Vazquez. (2020). "Prescribed-time estimation and output regulation of the linearized Schrödinger equation by backstepping". European Journal of Control. DOI: https: //doi.org/10.1016/j.ejcon.2020.02.009.

Tucsnak, M. and G. Weiss. (2009). Observation and Control for Operator Semigroups. Birkhauser. 
Tuna, S. E. and A. R. Teel. (2004). "Discrete-time homogeneous Lyapunov functions for homogeneous difference inclusions". In: 43rd IEEE Conference on Decision and Control (CDC). Vol. 2. 1606-1610. DOI: 10.1109/CDC.2004.1430274.

Utkin, V. I. (1992). Sliding Modes in Control Optimization. Berlin: Springer-Verlag.

Wang, S., A. Polyakov, and G. Zheng. (2020a). "Generalized homogenization of linear controllers: Theory and experiment". International Journal of Robust and Nonlinear Control. URL: https://doi.org/10. $1002 /$ rnc.5112.

Wang, X., S. Huang, and Z. Xiang. (2020b). "Output feedback finitetime stabilization of a class of nonlinear time-delay systems in the p-normal form". International Journal of Robust and Nonlinear Control. 30(11): 4418-4432. DOI: 10.1002/rnc.4996.

Watson, G. (1996). A Treatise on the Theory of Bessel Functions. Cambridge University Press.

Wonham, W. M. (1985). Linear Multivariable Control: A Geometric Approach. Springer.

Zimenko, K., D. Efimov, A. Polyakov, and A. Kremlev. (2019). "Independent of delay stabilization using implicit Lyapunov function method". Automatica. 101(3): 103-110.

Zimenko, K., D. Efimov, A. Polyakov, and W. Perruquetti. (2017). "A note on delay robustness for homogeneous systems with negative degree". Automatica. 79(5): 178-184.

Zimenko, K., A. Polyakov, and D. Efimov. (2018). "On finite-time robust stabilization via nonlinear state feedback". International Journal of Robust and Nonlinear Control. 28: 4951-4965.

Zimenko, K., A. Polyakov, D. Efimov, and W. Perruquetti. (2020). "Robust Feedback Stabilization of Linear MIMO Systems Using Generalized Homogenization". IEEE Transactions on Automatic Control.

Zubov, V. I. (1964). Methods of A.M. Lyapunov and Their Applications. Noordhoff, Leiden.

Zubov, V. (1958). "On systems of ordinary differential equations with generalized homogenous right-hand sides". Izvestia vuzov. Mathematica (in Russian). 1: 80-88. 